\title{
WING/WALL AERODYNAMIC INTERACTIONS IN FREE FLYING, MANEUVERING MAVS
}

\author{
Thesis \\ Submitted to \\ The School of Engineering of the \\ UNIVERSITY OF DAYTON
}

\begin{abstract}
In Partial Fulfillment of the Requirements for
The Degree of

Master of Science in Aerospace Engineering
\end{abstract}

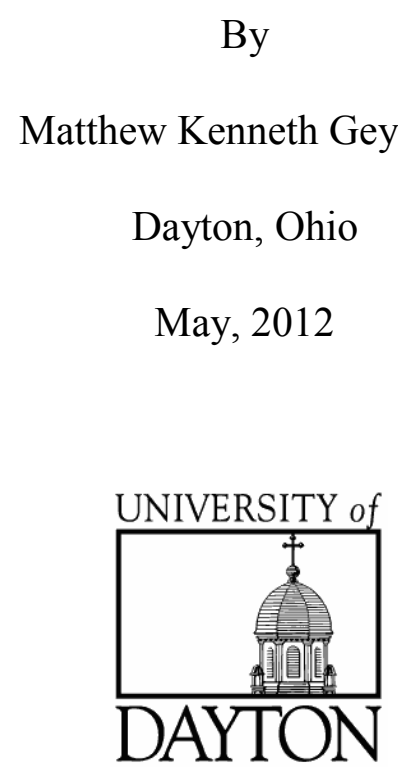




\title{
WING/WALL AERODYNAMIC INTERACTIONS IN
}

\author{
FREE FLYING, MANEUVERING MAVS
}

Name: Geyman, Matthew Kenneth

APPROVED BY:

Aaron Altman, Ph.D.

Gregory H. Parker, Ph.D.

Advisory Committee Chairman

Committee Member

Associate Professor and

AFRL/RBAL Air Vehicles Directorate

Director of Graduate Aerospace Engineering

MAV Team Lead

Department of Mechanical and

Aerospace Engineering

Markus Rumpfkeil, Ph.D.

Committee Member

Professor

Department of Mechanical and

Aerospace Engineering

John G. Weber, Ph.D.

Associate Dean

School of Engineering
Tony E. Saliba, Ph.D.

Dean, School of Engineering

\& Wilke Distinguished Professor 


\title{
ABSTRACT
}

\author{
WING/WALL AERODYNAMIC INTERACTIONS IN \\ FREE FLYING, MANEUVERING MAVS
}

Name: Geyman, Matthew Kenneth

University of Dayton

Advisor: Dr. Aaron Altman

Micro Air Vehicles (MAVs) are small remotely piloted air vehicles that can be flown between or inside of buildings for military or surveillance purposes. This type of flight in the urban environment involves many aerodynamic hazards. The research in this thesis investigates how the aerodynamic interactions between a maneuvering MAV's wingtip vortex and its distance away from a building wall could affect the MAV's flight controls. Free flight particle image velocimetry (PIV) testing and wind tunnel testing are used to investigate the aerodynamic interactions between a MAV wingtip vortex and a wall. Elliptical instabilities and a vortex rebound off of the wall are discovered in the PIV testing while the wind tunnel results show a higher aircraft coefficient of lift near the wall. All of these results force the aircraft to experience a rolling motion while flying along a wall. It is imperative that a MAV anticipate this motion and adjust its flight controls in order to accurately fly along a wall and successfully complete its mission in an urban environment. 


\section{ACKNOWLEDGMENTS}

As I have journeyed through graduate school, I have not traveled alone. I would like to thank Mandy McDonough for her love, support, and patience throughout our relationship and especially during the time that we have both spent in graduate school.

I would also like to thank Dr. Aaron Altman for his support and guidance

throughout my college career. He has helped me challenge myself, serve my community, develop my academic abilities, and become a well-rounded young professional. I would also like to extend my gratitude to the Altman family including Servane, Samuel, Eloise, and Melodie.

Dr. Greg Parker has also been a great support and example throughout my 3 year co-op rotation with the Micro Air Vehicle Integration and Application Research Institute. I have thoroughly enjoyed helping to develop a world class research facility and pushing the limits of MAV research. I also thank AFRL for the tuition assistance.

I would also like to thank Dr. Markus Rumpfkeil for his support in both my classes as well as my research. He truly taught me lessons about numerical methods that I will carry with me throughout my engineering career. I am grateful to have this knowledge and to have had him as a teacher. 
Finally, I would like to thank my fellow graduate students Zach Lego, Sidaard Gunasekaran, Ben Hagen, Patrick Hammer, Phil Ritenour, Danielle Christensen, Jon Dekar and all of the volunteers who donated their time when many hands were needed for the PIV testing.

Last but not least, I would like to thank my parents for their loving support throughout my life. They have always supported me in anything that I have been involved with and I will always be grateful for what they have done to help me get to this point in my life. 


\section{TABLE OF CONTENTS}

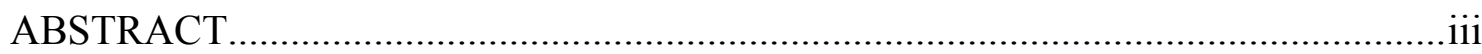

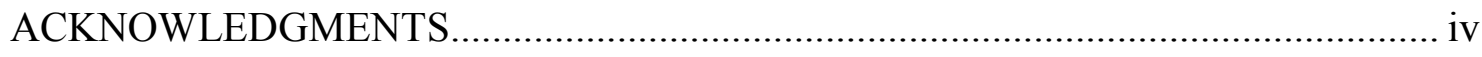

TABLE OF CONTENTS.............................................................................

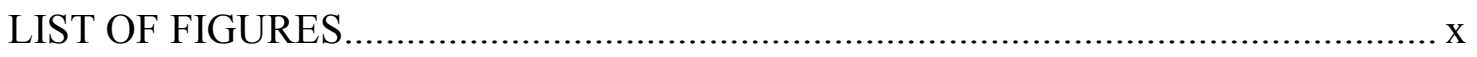

LIST OF TABLES.................................................................................................

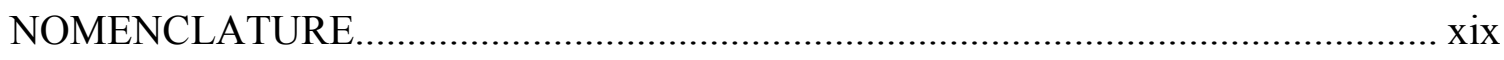

CHAPTER 1 - INTRODUCTION................................................................... 1

1.1 Micro Air Vehicle (MAV) Research...................................................... 1

1.2 MAV Flight in the Urban Environment.............................................. 2

1.3 Atmospheric Turbulence for MAVs in the Urban Environment......................2

1.4 Obstacles in the Urban Environment......................................................

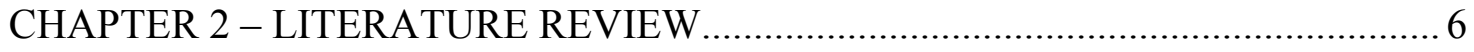

2.1 Aircraft Wake ....................................................................................... 6

2.2 Wingtip Vortex Formation.................................................................. 7

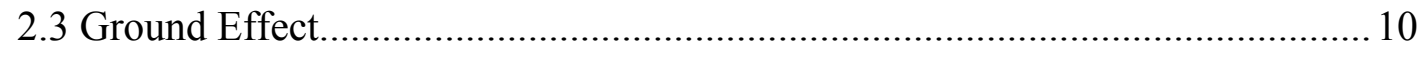

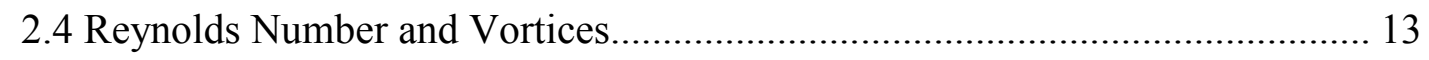

2.4.1 Low Reynolds Number and Vortices.............................................. 14 
2.4.3 Reynolds Number Sensitivity for Free Vortex Propagation.................. 15

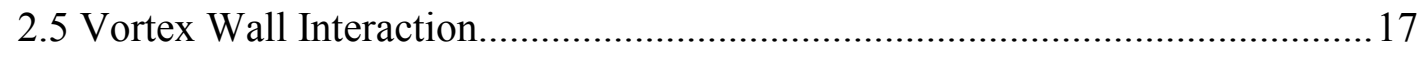

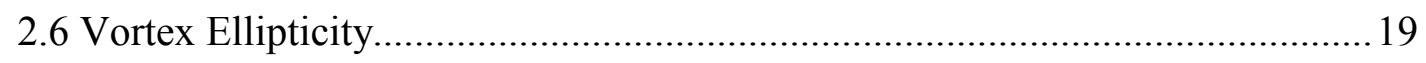

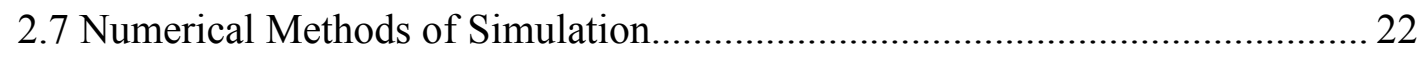

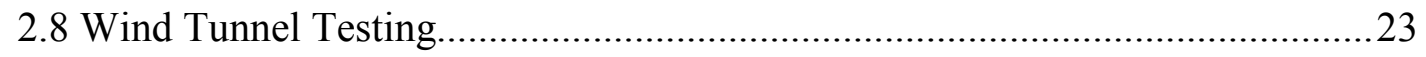

2.9 Free Flight Vortex Wake Experiment for a MAV Sized Bird........................... 28

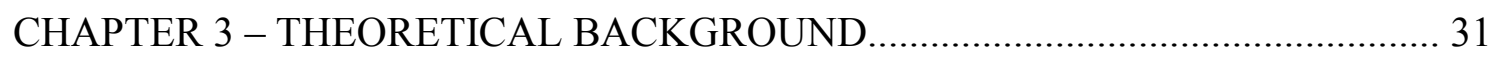

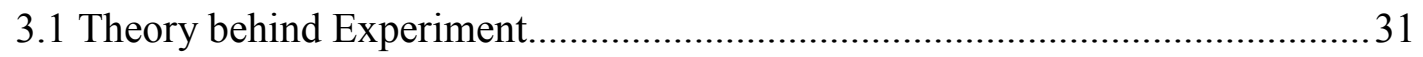

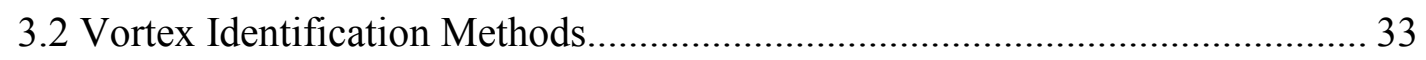

3.3 Methods of Calculating the Circulation of a Vortex............................................. 39

3.4 Methods of Calculating the Lift Force from a Vortex.........................................41

3.5 Wingtip Vortex Ellipticity.......................................................................... 43

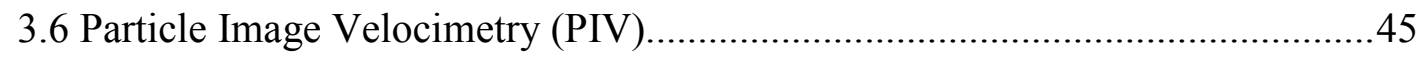

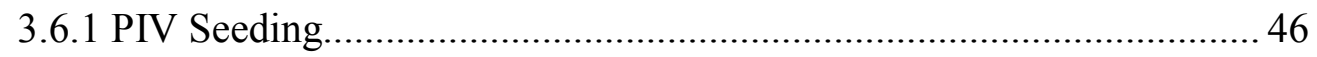

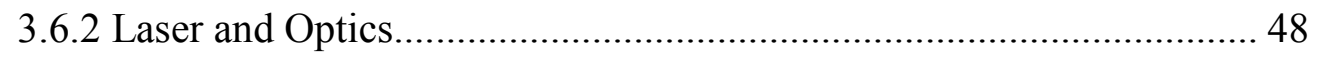

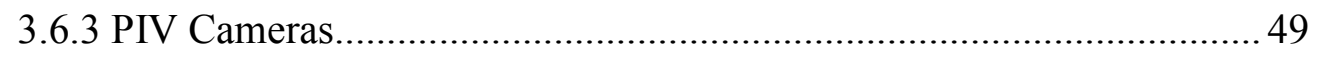

3.7 Micro Air Vehicle Indoor Flight Facility.....................................................50

3.8 Wind Tunnel Testing...............................................................................5

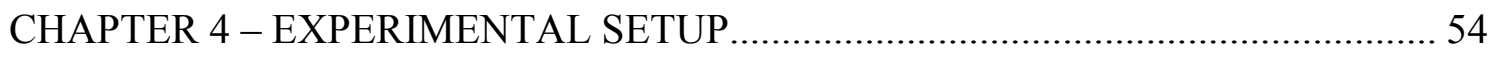

4.1 PIV Proof of Concept Experimental Setup....................................................... 54

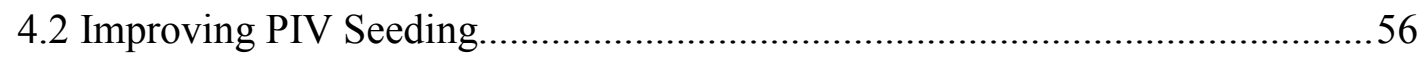

4.3 Final PIV Experimental Setup....................................................................57 


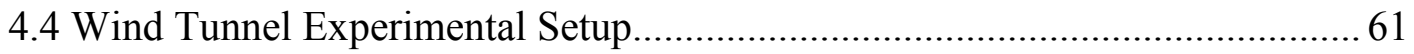

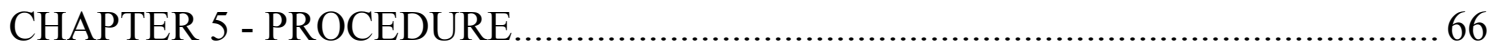

5.1 Proof of Concept PIV Procedure..................................................................66

5.2 Final PIV Testing Procedure .......................................................................... 67

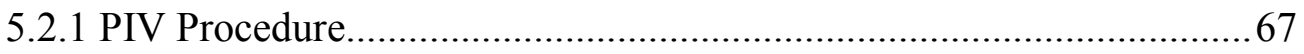

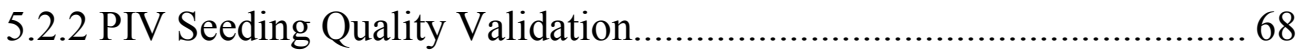

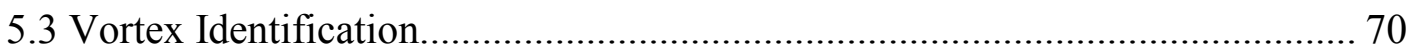

5.4 Calculating Vortex Circulation.................................................................... 76

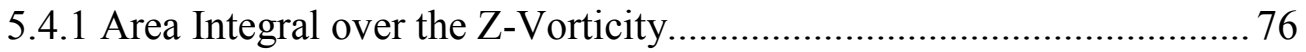

5.4.2 Line Integral of the Velocity over a Closed Curve................................ 78

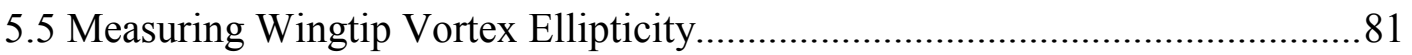

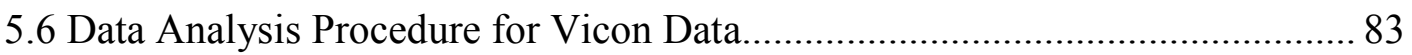

5.7 Wind Tunnel Testing Procedure ........................................................................84

5.7.1 Night Vapor Wind Tunnel Testing Procedure: Knowledge Gained..... 85

5.7.2 Wind Tunnel Data Processing Procedure............................................ 88

5.7.3 Uncertainty Analysis............................................................................91

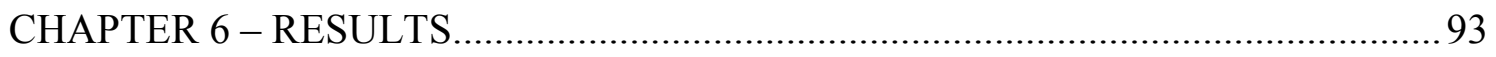

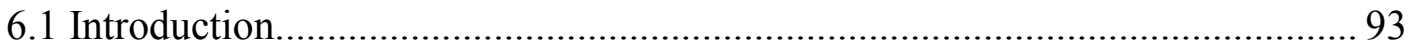

6.2 PIV Free Flight Testing................................................................................93

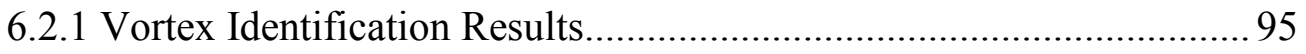

6.2.2 Wingtip Vortex Circulation Results......................................................96

6.2.3 Wingtip Vortex Ellipticity................................................................... 104

6.2.4 Secondary Vortex due to Wall Effect................................................... 109 


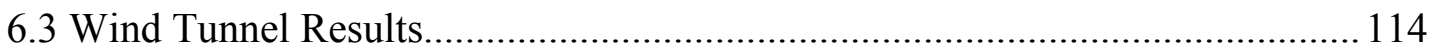

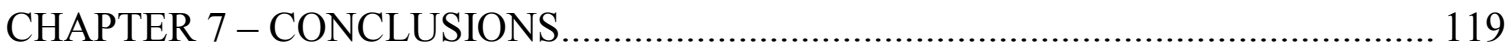

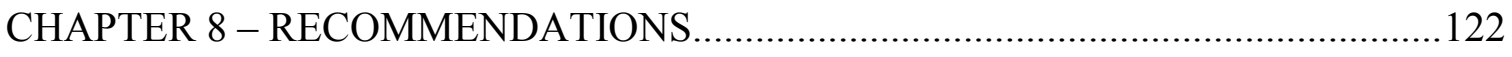

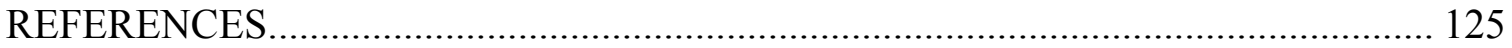

APPENDIX A- ADDITIONAL PIV INFORMATION................................................. 129

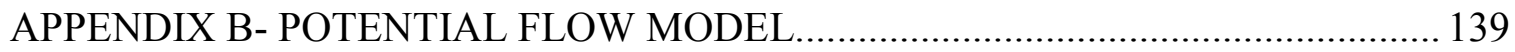

B.1 Potential Flow Model Simulation Theoretical Background................................ 139

B.2 Potential Flow Model Experimental Setup..........................................................141

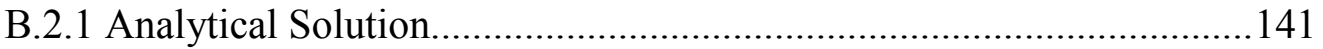

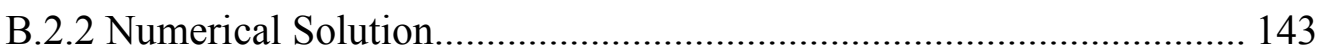

B.3 Potential Flow Model Procedure..................................................................... 146

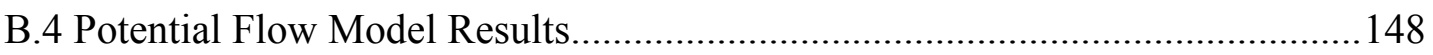

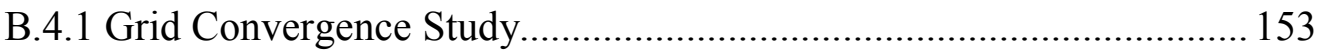




\section{LIST OF FIGURES}

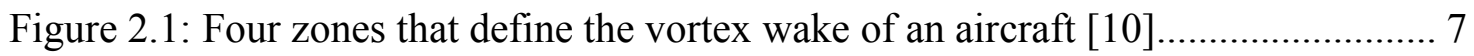

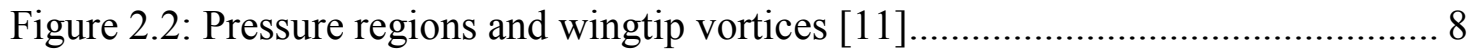

Figure 2.3: Bend in streamlines creating wingtip vortices. Dashed lines are under the

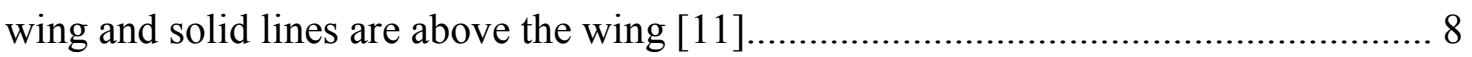

Figure 2.4: The physics involved with the rollup of a wingtip vortex [12]................. 9

Figure 2.5: Wake planform studies show that the wake has not experienced complete

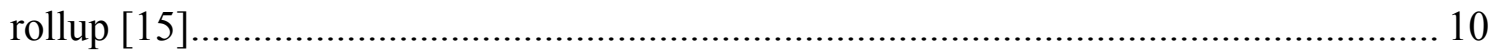

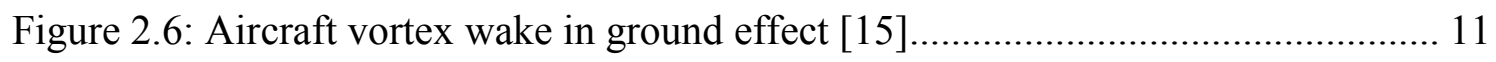

Figure 2.7: Ground effect on inducted drag ratio [15] ........................................ 11

Figure 2.8: Normalized turbulence, $\epsilon^{*}$, between the vortex cores for the C-130, B757,

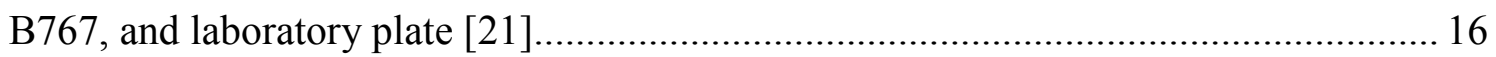

Figure 2.9: Vortex dipole interactions with a wall without any control parameters [22]

Figure 2.10: Contour plot of the vorticity of the vortex dipole as it impacts the wall at a $75^{0}$ angle [23]

Figure 2.11: Contour plot of the vorticity of the vortex dipole as it impacts the wall at a $45^{0}$ angle [23]. 19

Figure 2.12: Left to right: high, moderate, and low ellipticity for ideal two-dimensional 
vorticity Stuart model vortices [25].

Figure 2.13: Left to right: high, moderate and low ellipticity spanwise vorticity fields showing the instabilities present in each type of vortex [25]......

Figure 2.14: Contour plots of the vorticity of the vortex dipole as it interacts with a wall over nondimensional time $[26]$

Figure 2.15a: Dimensions of the cross section of the wind tunnel test section with the wall insert [27]

Figure 2.15b: Tunnel cross section with wall insert. Dimension "a" ranging from 1, 2, 4, and 5 in [27] .24

Figure 2.16: The average location of the wingtip vortex core with varying tip-to-wall distances [27] 25

Figure 2.17: Distance from wall to wingtip vs. the mean vortex core elevation. Alpha $=$ $5^{0}$ and $x=4 c[27]$ .25

Figure 2.18: Various vortex core radius sizes as well as different vortex vorticity values

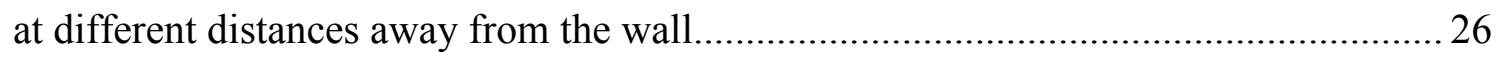

Figure 2.19: $\mathrm{C}_{\mathrm{Lmax}}$ and aspect ratio values for four wing planforms [28]....................28

Figure 2.20: $\alpha_{\text {CLmax }}$ and aspect ratios for the four wing planforms tested [28]..............28

Figure 2.21: Test setup for wake measurements from a kestrel gliding through a hallway [29]. 29

Figure 2.22: Corkscrew shape of a wingtip vortex can clearly be seen [29]. 29

Figure 3.1: Location of variables used in the $\Gamma_{1}$ vortex core identification method [32]......

Figure 3.2: Results of the $\Gamma_{2}$ method used to identify the boundary of a vortex [31].....37 
Figure 3.3: Results of the $\Gamma_{2}$ method used to identify the boundary of a vortex with $30 \%$ noise added [32] 37

Figure 3.4: Contours for vortex centers from $\Gamma_{1}$ and the vortex boundaries from $\Gamma_{2}$

Figure 3.5: Comparison of results for two methods used to calculate the circulation for a vortex [33] 40

Figure 3.6: Variation of control volume for circulation calculations [33].....................40

Figure 3.7: Flow chart showing the theory behind a PIV test setup [40] ..................... 46

Figure 3.8: RMS-uncertainty vs. the particle image diameter for cross correlation PIV $[41]$

Figure 3.9: Flight room, Vicon camera example, and Vicon software to show virtual

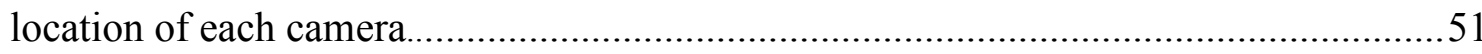

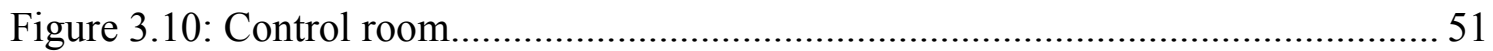

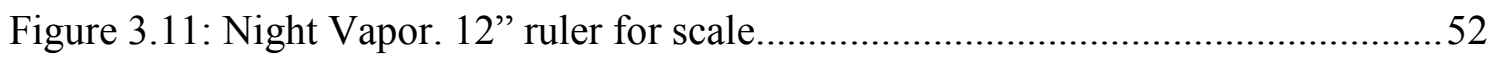

Figure 3.12: Reflective markers on aircraft as seen in Vicon software ........................52

Figure 4.1: Test setup in the middle of the room. The brown cardboard box around the laser, optics, and smoke machine, directed the smoke into the laser sheet....................56 Figure 4.2: The laser, $45^{\circ}$ mirror, optics, and smoke machine are shown here with the box

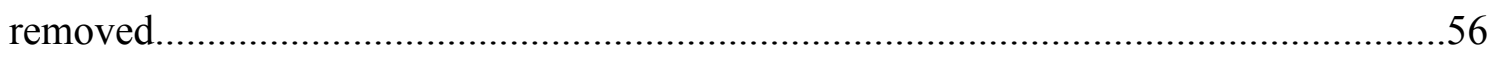

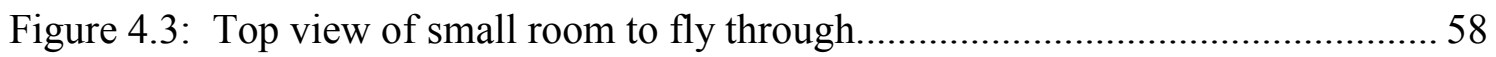

Figure 4.4: Side view of wall test setup in flight room...........................................58

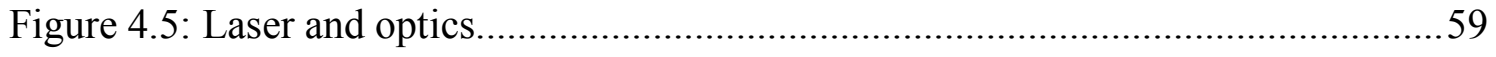

Figure 4.6: Test setup to contain smoke but allow the aircraft to fly through the room 
without the wall having a significant effect on aircraft wingtip vortex.

Figure 4.7: Flying through laser: far away from wall.

Figure 4.8: UD low speed wind tunnel. Close up image of fan inserted for reference...61

Figure 4.9: X-EC attached to the sting force balance. 63

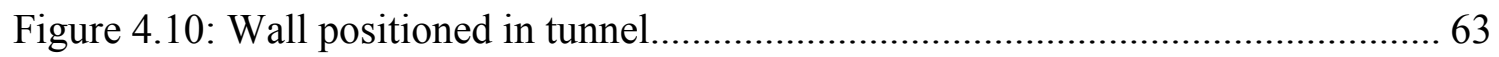

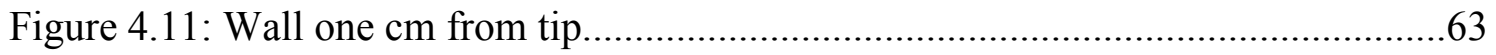

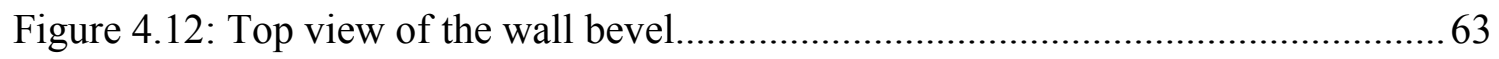

Figure 4.13: Interface between sting and aircraft mount for both Vapor and X-EC....... 64

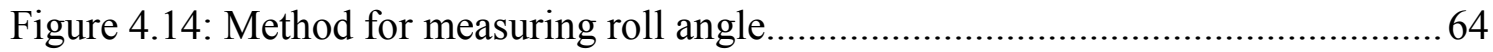

Figure 5.1: Full scale raw PIV image of the smoke particles within the field of view...69

Figure 5.2: Zoomed in image of the smoke particles in Figure 5.1............................. 69

Figure 5.3: 8-10 ideal pixel shifts for wingtip vortices is achieved for a fast straight test

without a wall.

Figure 5.4: Location of variables used in the $\Gamma_{1}$ vortex core identification method

Figure 5.5: Z-vorticity contour plot of a wingtip vortex for a fast straight flight near the

wall.

Figure 5.6: Streamlines plotted to help determine the size of the Msub mesh. 72

Figure 5.7: Zoomed in image of white Msub mesh and black Psub mesh. 74

Figure 5.8: Vorticity magnitude and normalized velocity contour plots showing the vortex center and vortex boundaries.

Figure 5.9: Multitude of area integration boxes for circulation calculation sensitivity analysis. .78 
Figure 5.10: Integration box for area integration circulation calculation

Figure 5.11: Streamline that is used for the line integration circulation calculation....... 79

Figure 5.12: Discontinuity in beginning and termination of a streamline..................... 79

Figure 5.13: Linear interpolation helps the first two and last two points line up with the

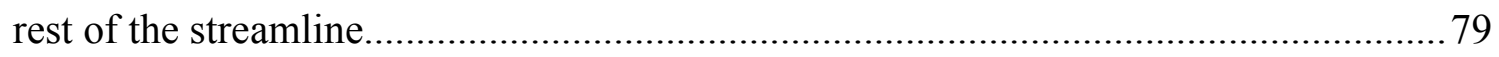

Figure 5.14: Circular vortex from a fast straight test without wall present................... 82

Figure 5.15: Elliptical vortex from a slow turning wall test..................................... 82

Figure 5.16: $C_{L}$ vs. alpha curves and lift curve slope equations for the experimental and

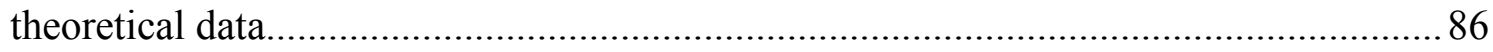

Figure 5.17: Free body diagram of the sting force balance as viewed from the side..... 89

Figure 5.18: Free body diagram of the airplane wing (red) as viewed from head on......90

Figure 6.1: Noisy velocity vectors are seen in the proof of concept PIV testing........... 95

Figure 6.2: Final PIV velocity vector field shows clear vortices with few noisy vectors

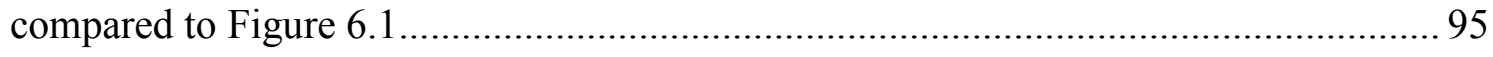

Figure 6.3: This area integration circulation sensitivity analysis plot shows a trend of increasing circulation values with increasing integration area until the circulation

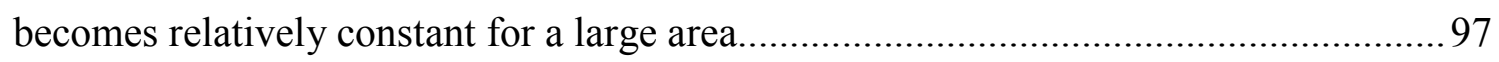

Figure 6.4: This normalized area integration circulation sensitivity analysis shows that the normalized circulation increases with the constantly increasing normalized radial position within the vortex boundaries .100

Figure 6.5: The trend of constantly increasing normalized circulation with constantly increasing normalized radial position for trailing vortices can be seen in this plot [46]. 102 Figure 6.6: Similar average raw circulation values are seen for the area and line 
integration circulation calculations for wingtip vortices in this thesis research.

Figure 6.7: This plot of the ellipticity values for each test condition shows that the

wingtip vortices near a wall always have a higher ellipticity value.

Figure 6.8: The dominant instability and the weaker edge instability are shown in this normalized velocity contour plot from a slow turning flight near the wall.

Figure 6.9: Normalized velocity contour plot for a wingtip vortex from a slow straight flight without the wall shows a lack of instabilities.

Figure 6.10: The yellow line shows the vertical major axis of the elliptical shaped wingtip vortex near a wall. Secondary vortex also identified.

Figure 6.11: The velocity vectors in this image form a more circular shape compared to that of Figure 6.10 since a wall is not present in this test. 109

Figure 6.12: Top Row: Time evolution of the wingtip vortex as seen in the velocity vector field plots. The vortex core moves up and away from the wall as indicated by its position above the white line. Bottom Row: Nondimensional time evolution of vortex dipole particles impacting a wall from a particle-in-cell method by Ho Liu and Doorly [23]. This shows a similar secondary vortex develop. 110

Figure 6.13: Formation of the secondary vortex due to separation.

Figure 6.14: Fast straight and slow straight wind tunnel results show an increase in $\mathrm{C}_{\mathrm{L}}$ as the wall approaches the wingtip. 115

Figure 6.15: Fast turning and slow turning wind tunnel results show an increase in $\mathrm{C}_{\mathrm{L}}$ as the wingtip nears the wall. 115

Figure A.1: Plots of the wingtip vortices from a fast straight flight without the wall.....129

Figure A.2: Plots for a wingtip vortex from a slow straight test without the wall. 
Figure A.3: Velocity vector field plot, normalized velocity contour, and z-vorticity contour plot of the wingtip vortices from a fast turning flight without the wall.

Figure A.4: Velocity vector field plot, normalized velocity contour, and z-vorticity contour plot of the wingtip vortices from a slow turning flight without the wall.

Figure A.5: Velocity vector field plot, normalized velocity contour, and z-vorticity contour plot of the wingtip vortex from a fast straight flight along the wall.

Figure A.6: Velocity vector field plot, normalized velocity contour, and z-vorticity contour plot of the wingtip vortex from a slow straight flight along the wall.

Figure A.7: Velocity vector field plot, normalized velocity contour, and z-vorticity contour plot of the wingtip vortex from a fast turning flight along the wall

Figure A.8: Velocity vector field plot, normalized velocity contour, and z-vorticity contour plot of the wingtip vortex from a slow turning flight along the wall.

Figure A.9: Wingtip vortices are seen in these proof of concept testing images as the Night Vapor leaves the field of view. The PIV seeding is not ideal for analysis but clearly shows the formation of wingtip vortices

Figure A.10: Normalized circulation values for three different fast straight tests with and without the wall present.

Figure A.11: Normalized circulation profiles for three slow straight tests with and without the wall present. 135

Figure A.12: Normalized circulation profiles for three fast turning tests with and without the wall present. 135

Figure A.13: Normalized circulation profiles for three slow turning tests with and without the wall present. 136 
Figure A.14a: Slow turn test two without wall z-vorticity contour plot. The large amount of spurious z-vorticity seen in this image contributes to the larger normalized circulation values seen in Figure A.15.

Figure A.14b: Slow turn test three with the wall present. Spurious z-vorticity is not seen in this image and the normalized circulation values are similar to the literature. See

Figure A.15.

Figure A.15: Sporadic noisy z-vorticity causes unreasonable spikes in the normalized circulation of these slow turning test circulation profiles. 138

Figure B.1: Convergence plot for norm of residual vs. number of iterations. .148 Figure B.2: Numerical and analytical stream function vortex contour plots look similar....

Figure B.3: Analytical and numerical stream function values for several interior rows and columns in the mesh. 150 Figure B.4a: The stretched shape of the analytical stream function vortex contour plot is due to the added vertical free stream flow...... .151 Figure B.4b: The circular shape of the analytical stream function vortex contour plot without the vertical free stream added can be seen.

Figure B.5: $\mathrm{X}$ and Y position with the magnitude of the analytical and numerical velocities plotted as contours.

Figure B.6: X distance vs. the difference in the analytical and numerical velocity magnitudes 153

Figure B.7: Plot of the grid convergence study results with the slope of the trend line included. .154 


\section{LIST OF TABLES}

Table 2.1: Chord and vortex Reynolds numbers for the laboratory experiment and full

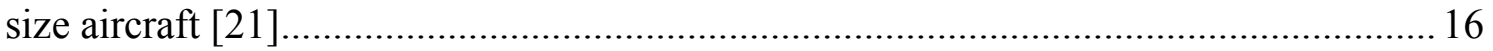

Table 3.1: Test matrix for free flight and wind tunnel tests.......................................33

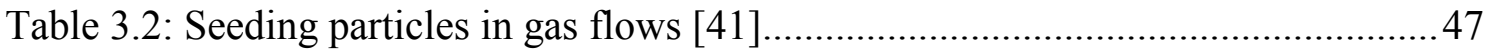

Table 3.3: Details for the ParkZone Night Vapor RC aircraft.....................................52

Table 5.1: Values used in wingtip vortex circulation estimation................................80

Table 5.2: Omega pressure transducer error analysis................................................. 92

Table 6.1: Percentage rise in circulation focused on three areas around the vortex where

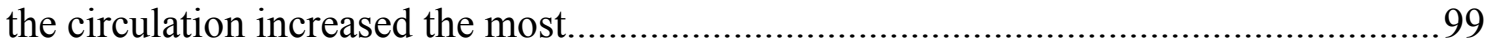

Table 6.2: When comparing the percentage of increase in $\mathrm{C}_{\mathrm{L}}$ for different maneuvers a higher percentage of increase in $\mathrm{C}_{\mathrm{L}}$ exists near the wall..................................... 116 Table 6.3: Highest wing loading and vertical gust velocities are seen for the fast turning

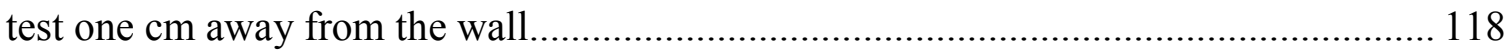

Table B.1: Grid convergence results. The error reduces with more grid points............. 154 


\section{NOMENCLATURE}
A
Wing planform area
a
Distance between vortex core and wall
$\alpha$
Aircraft angle of attack
AR
Aspect ratio
b
Span of a wing, circulation of the wingtip vortex in Maple code
c
Chord of wing
$\mathrm{C}_{\mathrm{D}}$
Coefficient of Drag
$C_{L \alpha 2 D \text { airfoil }}$
Lift curve slope of a symmetric two-dimensional airfoil with a $2 \pi$
lift curve slope
$\mathrm{C}_{\mathrm{L}}$
Coefficient of lift
D
Total drag force
$\mathrm{D}_{\mathrm{N}}$
Drag due to normal force
$\mathrm{D}_{\mathrm{A}}$
Drag due to axial force
dl
Closed curve
dS
Area of a vortex
e
Oswald efficiency factor
$\mathrm{F}_{\mathrm{A}}$
Axial force from sting - average tare axial force 


\begin{tabular}{|c|c|}
\hline $\mathrm{F}_{\mathrm{N}}$ & Normal force from sting - average tare normal force \\
\hline $\mathrm{F}_{\mathrm{na}}$ & Component of side force that is normal to the aircraft \\
\hline Fs & Side force \\
\hline $\mathrm{g}$ & Stream function in Maple code \\
\hline$\Gamma_{\mathrm{z}}$ & Circulation of a vortex \\
\hline$\Gamma_{1}(P)$ & A dimensionless scalar used to pinpoint the vortex core center \\
\hline $\mathrm{L}$ & Total lift force \\
\hline $\mathrm{L}_{\mathrm{A}}$ & Lift due to axial force \\
\hline $\mathrm{L}_{\mathrm{N}}$ & Lift due to the normal force \\
\hline$\varphi$ & Roll angle \\
\hline $\mathrm{L} \varphi$ & Component of the side force that is normal to the balance \\
\hline $1_{0}$ & Length of a typical vortex inner core \\
\hline M & $\begin{array}{l}\text { The point surrounding point } P \text { where the velocity vector } U_{M} \text { is } \\
\text { located }\end{array}$ \\
\hline $\mathrm{N}$ & The number of points $\mathrm{M}$ inside of $\mathrm{S}$ \\
\hline $\mathrm{P}$ & The location where $\Gamma_{1}(P)$ and $\Gamma_{2}(P)$ is calculated \\
\hline PM & Radius vector from point $\mathrm{P}$ to point $\mathrm{M}$ \\
\hline$\rho$ & Density of air \\
\hline $\operatorname{Re}$ & Reynolds Number \\
\hline $\mathrm{r}$ & Vortex core radius of individual vortex \\
\hline $\mathrm{r}_{0}$ & Vortex core radius for a typical vortex \\
\hline S & The two dimensional area surrounding point $\mathrm{P}$ \\
\hline$\psi$ & Stream function \\
\hline
\end{tabular}




\begin{tabular}{|c|c|}
\hline$\theta_{M}$ & The angle between the velocity vector $U_{M}$ and the radius vector \\
\hline & $\mathrm{PM}$ \\
\hline $\mathrm{u}$ & $\mathrm{X}$ component of velocity \\
\hline $\mathrm{U}_{\mathrm{M}}$ & The velocity vector around the vortex \\
\hline$\widetilde{U_{P}}$ & The local convection velocity at point $\mathrm{P}$ \\
\hline$U_{\theta}(r)$ & Velocity dependent of the vortex core radius \\
\hline $\mathrm{Vr}$ & Vortex radius \\
\hline $\mathrm{V}$ & Velocity \\
\hline $\mathrm{V}_{\infty}$ & Free stream velocity \\
\hline $\mathrm{V}$ & Y component of velocity \\
\hline$\omega_{\mathrm{z}}$ & Z-vorticity \\
\hline $\mathrm{x}$ & Cartesian coordinate system for mesh \\
\hline $\mathrm{y}$ & Cartesian coordinate system for mesh \\
\hline Z & $\begin{array}{l}\text { Elevation of the aircraft / Unit vector normal to the measurement } \\
\text { plane }\end{array}$ \\
\hline$\wedge$ & $\begin{array}{l}\text { This symbol represents an exterior product, which is a cross } \\
\text { product of PM and } U_{M}\end{array}$ \\
\hline
\end{tabular}




\section{CHAPTER 1}

\section{INTRODUCTION}

\subsection{Micro Air Vehicle (MAV) Research}

There are many design challenges associated with developing a successful MAV and a variety of scientific disciplines are needed to make advances within this field. The primary application for a MAV involves remotely flying it through complicated urban areas for surveillance and security purposes for military or police forces. Abate et al [1] explain that in order to complete such a task it is necessary to combine the power of research, computational simulation, and experimentation. All of these approaches will work together to continue to make advances in the field of MAVs. Abate et al [1] provide an overall view of the complex subjects that will need to be studied and understood in order to help MAV technology advance.

But to enable conceptual breakthroughs in the design of MAVs/NAVs with flight-hover-perch capabilities under unpredictable wind-gust influence and in urban environments with complicated flight paths that are subject to real-time corrections, qualitatively improved understanding of the fluid mechanics, fluid-structure interactions, nonlinear control, and, ultimately, integrated capabilities with the combined aerodynamicsstructural-control approach need to be attained [1].

The research presented in this thesis focuses on furthering the knowledge and understanding of the complex aerodynamics involved with MAV flight in the urban 
environment. At present, for fixed wing MAVs there is little data on precisely how close to walls they can fly or maneuver, or what the aerodynamic effects of doing so are on the control of the vehicle. It is the goal of this proposed effort to investigate these problems so that the results can be used in the future to validate simplified models. These models will be used for the study of the design and real-time control of MAVs maneuvering around buildings. This is important because aerodynamic hazards between the MAV and the building could make it difficult to accurately control the MAV.

\subsection{MAV Flight in the Urban Environment}

Flight in the urban environment provides many challenges for MAVs. In a paper by Orr et al [2] the aerodynamics of urban environments in general and the challenges associated with MAVs flying in these urban environments are addressed. A virtual urban environment is developed which allows three-dimensional wind data to be analyzed as it flows between buildings and creates a complex accelerated flow containing vortices [2]. This type of flow will have a detrimental effect on a slow flying MAV and it is important for MAVs to be able to compensate for these aerodynamic effects as a result of flying near obstructions. The wall effects research in this thesis will investigate such aerodynamic effects since the urban environment creates many hazards for MAVs.

\subsection{Atmospheric Turbulence for MAVs in the Urban Environment}

In order to understand how a MAV will interact with its surroundings the dynamic sensitivity of the MAV to atmospheric turbulence also needs to be understood. This subject is investigated in a paper by Abdulrahim et al [3]. "In many cases, the 
vehicle designs and control systems are tested and tuned in calm-wind, open-atmosphere flight, away from turbulence-induced landscape features"[3]. The research throughout the rest of this thesis will eventually be used to develop predictive algorithms for closed loop flight controls to help MAVs autonomously navigate when flying in close proximity to walls.

Watkins et al [4] explain how to replicate atmospheric turbulence inside of a wind tunnel in order to test how MAVs interact with this type of turbulence. "Our study is restricted to generating well mixed or random turbulent flow, rather than investigating discrete gusts and local effects (such as might be found in extremely close proximity to buildings etc)" [4]. This statement highlights the gap that will be partially filled by the research performed in this thesis through quantifying the local effects of a wingtip vortex and a wall on the airplane. The knowledge gained from this research of MAVs flying past walls could also be extended to investigate how a MAV may interact with a variety of obstacles in its environment.

\subsection{Obstacles in the Urban Environment}

Flying a MAV close to the ground is just one type of obstacle a MAV will face in the urban environment. In a different paper, Watkins et al [5] explain that the main challenge with flying close to the ground involves the fact that the intensity of turbulence increases as an aircraft gets closer to the ground and aircraft stability becomes difficult to maintain. "MAVs will, however, have to fly in and around large obstacles as a matter of course and future measurements should examine the transient flow field experienced as a 
MAV traverses obstacle wakes in a given terrain and wind condition" [5]. However, the ground is only one of the obstacles a MAV will face in its environment.

Research has been conducted relating to rotorcraft flying in urban environments in close proximity to structures that simulate buildings. An example includes the research conducted by Shim et al [6] where map building and obstacle avoidance methods are investigated for helicopter unmanned air vehicles (UAVs). While this research is successful, the intent of this research did not include an aerodynamic analysis to study the interactions between the structures and the rotorcraft. Since the obstacles are canopy style tents, they did not have side walls and therefore did not have the capability to affect the aircraft in ways that would model a more representative urban environment.

Experimental obstacle avoidance technology is described by Kurdila et al [7]. Learning algorithms are successfully applied to a fixed wing MAV from the University of Florida and the aircraft is able to recognize two-dimensional terrains and plan and execute a flight path. A paper by Prazenica [8] implements and modifies these learning algorithms in a three-dimensional simulation code to improve them for three-dimensional object recognition and avoidance [8]. While this research helps a MAV recognize hazardous objects in its surrounding, the vehicle must also be able to plan for the aerodynamic interactions between the vehicle and its environment.

While recognizing obstacles when flying in the urban environment is important, it is also imperative for MAVs to be capable of flying aggressive maneuvers in order to avoid those objects. In a paper by Hall et al [9], both simulation and experimentation are used to program a MAV aircraft to perform loop and twist maneuvers which would specifically help a MAV avoid obstacles when flying in tight spaces [9]. While this 
research helps the MAV avoid obstructions the MAV must also be capable of taking the aerodynamic interactions into account as a result of flying past the objects in its environment. The research in this thesis will investigate the aerodynamic interactions between a MAV performing straight flights and turning maneuvers in close proximity to a wall. 


\section{CHAPTER 2}

\section{LITERATURE REVIEW}

\subsection{Aircraft Wake}

Studying the aerodynamic interactions between a fixed wing MAV and its surroundings will require analyzing the wake of the aircraft. A book by Ginevsky and Zhelannikov [10], explains that the wake of an aircraft can be divided into four sections to help understand the spatial-temporal evolution of the flow. Figure 2.1 shows the four zones trailing an aircraft. The wake formation zone involves the geometry of the aircraft creating complex flows that make up the wake. The stable wake zone contains vortices as they sink and gradually decay. The vortices begin to break down in the unsteady wake zone. The wake breakdown zone is made up of isolated vortex rings [10]. For the aerodynamic interactions between a fixed wing aircraft and a wall in this thesis, the vortices under consideration evolve into the stable wake zone. 


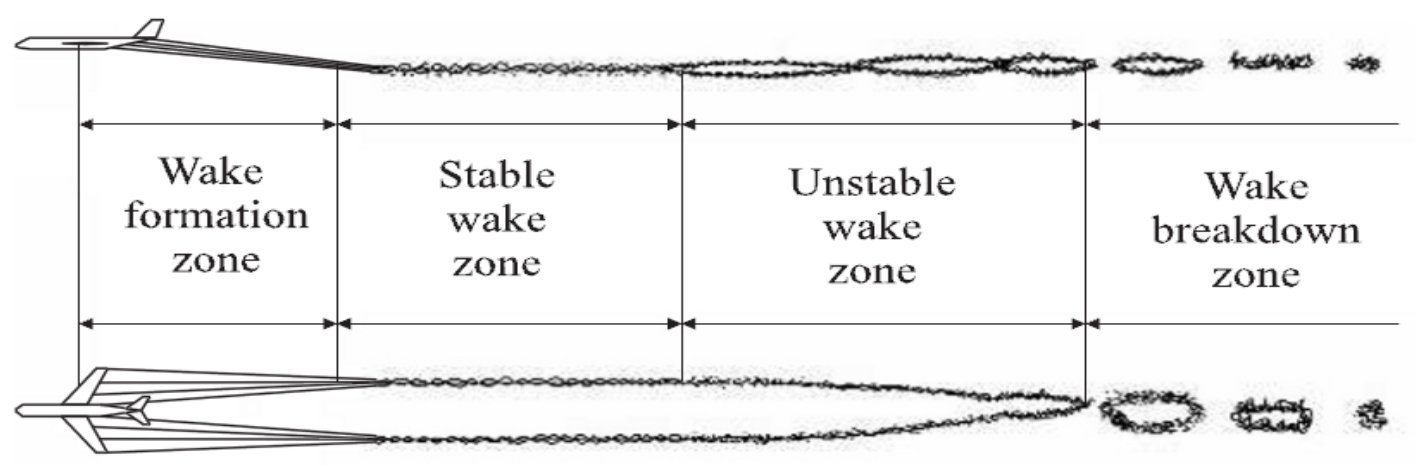

Figure 2.1: Four zones that define the vortex wake of an aircraft [10].

Ginevsky and Zhelannikov [10] go on to state that the vortex wakes of an aircraft are also affected by obstructions. They state that the interference of the vortex wake and vortex flow from buildings and rugged terrain create complex flow structures, wind shear, and flow velocities dangerous to an aircraft. These flows can be found around airports quite frequently. These types of interference can cause the unstable wake zone and the wake breakdown zones to merge together, causing an unsteady wake pattern [10]. This research demonstrates that the complex flow structures involved with aircraft wakes and their surroundings could easily compromise the success of a MAV mission while flying through complex terrain.

\subsection{Wingtip Vortex Formation}

In a paper by Hong and Altman [11], spanwise flow over a fixed aircraft wing and the creation of the wingtip vortex is explained. Figure 2.2 shows the regions of high and low pressure which help to explain the theory of lift. Figure 2.3 shows that the flow over the wings also has a spanwise component which causes the streamlines to alter their paths. The streamlines over the top of the wing tend to bend towards the root while the 
streamlines on the bottom of the wing tend to bend towards the tip of the wing. This alteration in the flow over and under the wing leads to the flow around the wingtips seen in Figure 2.2. This flow leaves the high pressure region, flows around the wingtips, and enters the low pressure region [11].
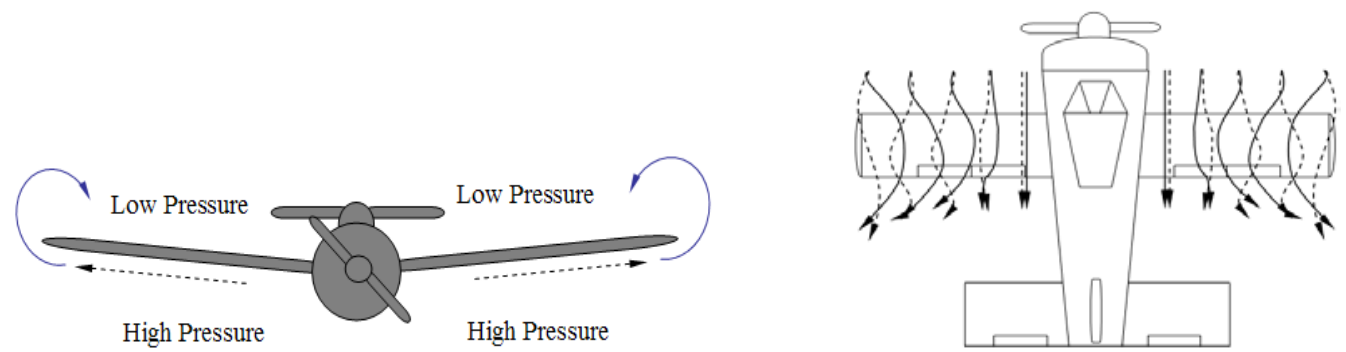

Figure 2.2: Pressure regions and wingtip vortices [11].

Figure 2.3: Bend in streamlines creating wingtip vortices. Dashed lines are under the wing and solid lines are above the wing [11].

Chow et al [12] also describe the physics behind how wingtip vortices are produced from a wing. See Figure 2.4. As the figure shows, streamlines pass above and below the wing and the vortex grows from the vorticity from the boundary layer near the wingtip. As seen by the helical streamlines in Figure 2.4, the vortex tube is created by the wingtip vortex rolling up the wing's wake [12]. 


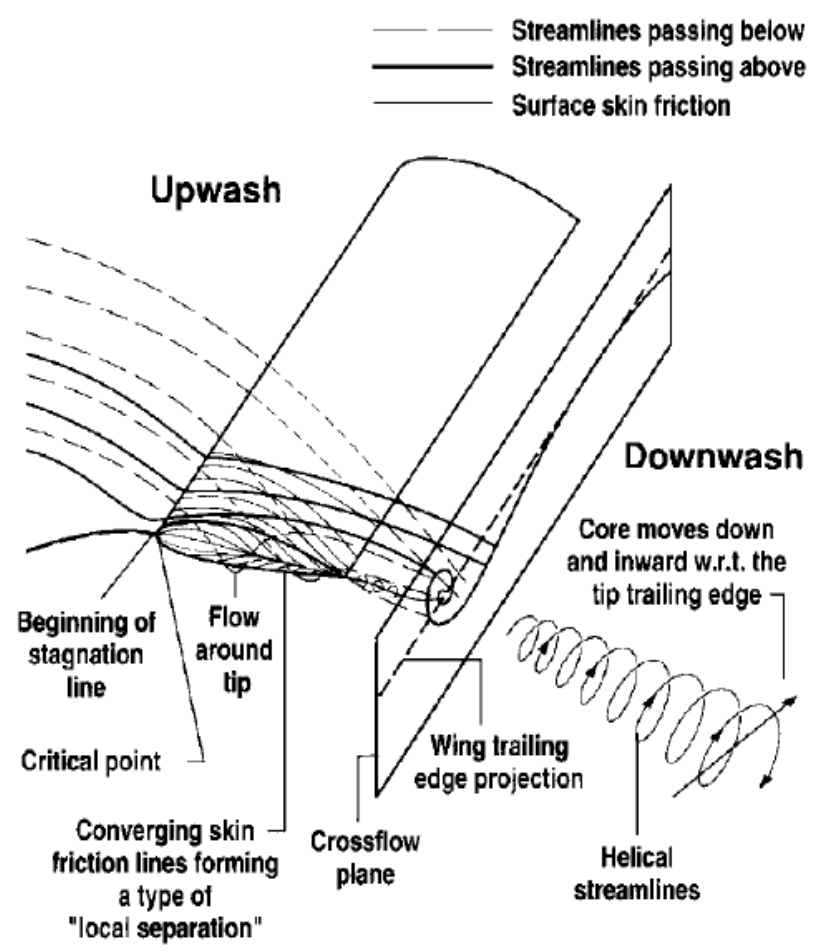

Figure 2.4: The physics involved with the rollup of a wingtip vortex [12].

Lian and Shyy discuss [13] how wingtip vortices can have an effect on an aircraft. The effective angle of attack decreases and the drag increases as a result of the wingtip vortex creating downwash for the membrane wing with a chord Reynolds number of $90,000[13,14]$.

Barnes [15] investigates the rate of wake-rollup for elliptical wings by studying the wake of pelicans flying in formation. The goal is to determine if the wake from the leading pelican completely rolls up before it reaches the trailing pelican. The results of this analysis can be seen in Figure 2.5 and it is determined that a trailing pelican located one or two wingspans downstream from the leading pelican will not experience significant wake-rollup [15]. Figure 2.5 also shows that the wake lines behind the wings have not significantly converged at the points of interest downstream from the wing. 
Barnes [15] also takes this wake analysis further and discusses how the wake interacts with ground effect.

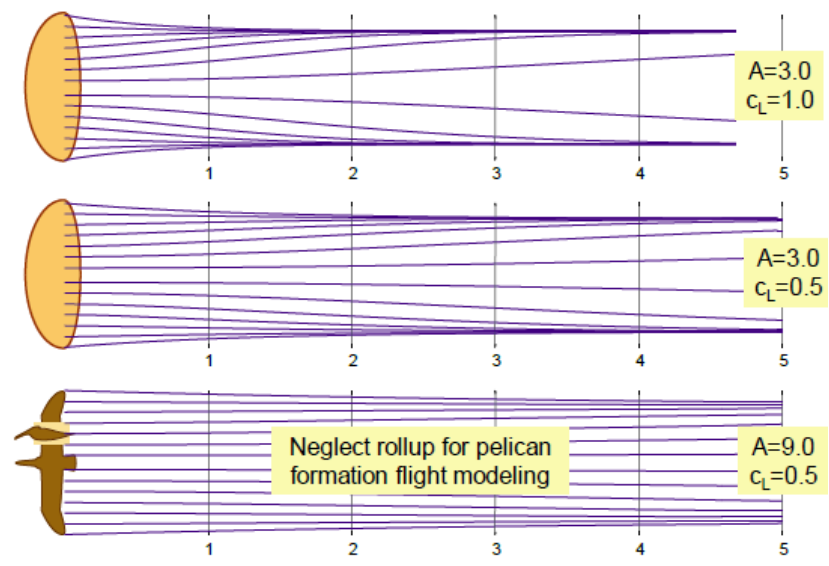

Figure 2.5: Wake planform studies show that the wake has not experienced complete rollup [15].

\subsection{Ground Effect}

Barnes [15] discusses ground effect by first describing how ground effect can be conceptually understood by visualizing a mirror image of the aircraft below the plane of the ground. This mirrored image of the aircraft has bound and trailing vortices rotating in the opposite direction compared to the actual aircraft wake vortices. The visualized aircraft representing the ground causes an upwash and streamwash on the actual aircraft. This results in an increase in lift, a reduction in airspeed, and a negative change in pitching moment. See Figure 2.6 [15]. 


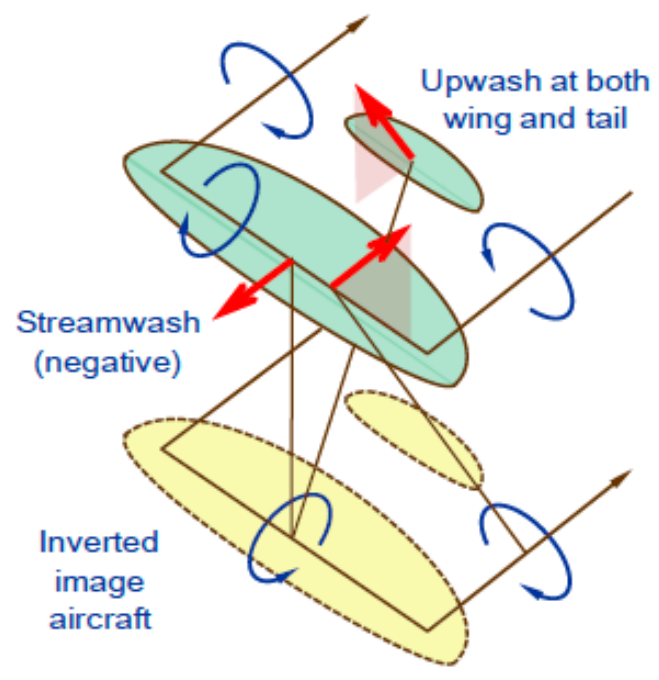

Figure 2.6: Aircraft vortex wake in ground effect [15].

The conclusions that Barnes [15] draws includes the following. "(a) Wingtips are unloaded as $[\mathrm{z} / \mathrm{b}]$ decreases and $(\mathrm{b})$ the induced drag reduction is independent of aspect ratio" [15]. In the first conclusion, $\mathrm{z}$ is the elevation of the aircraft and $\mathrm{b}$ is the span of the wing. The second conclusion can clearly be seen in Figure 2.7. The graph shows that the induced drag ratio decreases as the wing nears the ground [15].

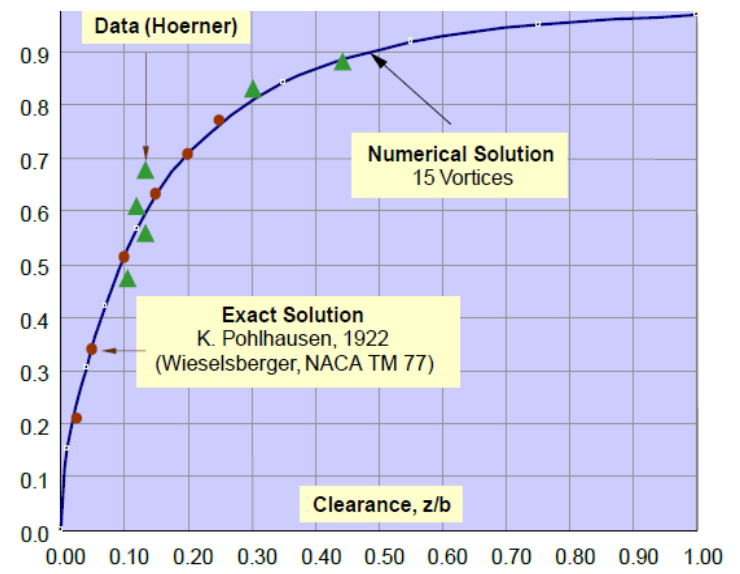

Figure 2.7: Ground effect on inducted drag ratio [15]. 
In a paper by Boschetti et al [16], stability and performance characteristics of a light unmanned aircraft in ground effect are investigated. As a wing approaches the ground, the induced drag decreases and the lift curve slope increases. The plane of the ground that the aircraft is flying along also reflects the trailing vortices in the aircraft's wake and causes a reduction in the vortices' strength. The presence of the ground also causes the vortices to induce an up-wash on the wing. This up-wash causes a reduction in the total induced incidence on the wing compared to a wing that is not in the presence of a ground plane. In classical ground effect theory, ground effects do not seem to have a significant effect on the lateral-directional stability of the aircraft, but it is expected that the longitudinal stability will be significantly affected by the presence of the ground. This is due to the fact that the downwash angle at the tail is less while flying along the ground when compared to flying away from the ground. This fact helps the tail to have a more significant contribution to the aircraft's stability since the lift curve slope of the horizontal tail grows [16].

After explaining the physics behind classical ground effects, Boschetti et al [16] discuss their research into the ground effects of a light unmanned airplane. The flow field around the aircraft's wing in close proximity to the ground is modeled with a vortex lattice and a panel method and it is concluded that the panel method can successfully model the lift and induced drag of the aircraft as a result of the ground effect. Overall, it is concluded that the chosen aircraft is statically unstable in the longitudinal direction while flying along the ground. A divergent and nonoscillatory mode develops as a result of ground effect and this is called a nondimensional height mode. This research also 
concludes that the Dutch roll, roll, and spiral modes are also slightly affected by ground effect [16].

The wall effects research relating to MAVs discussed throughout the rest of this thesis could be considered to involve similar concepts when compared to the physics behind ground effects. However, when an aircraft flies close to the ground, its entire wingspan is in close proximity to the ground. In contrast, when an aircraft flies close to a wall, only one wingtip is in close proximity to the wall. This means that in steady level flight the opposite wingtip is located at least an entire wingspan's distance away from the wall structure. The wall will not have a significant effect on this distant wingtip vortex, especially if the wing span is great enough so that this wingtip is located at least five wingtip vortex core diameters away from the wall. During ground effects, the presence of the ground causes a decrease in the wake vortices' strength. In wall effects, the wall can only affect one of the wingtip vortices. The longitudinal stability will also not be affected as much by wall effects since the downwash angle at the tail will not be as affected as would be experienced along the ground [16]. It is also important to note that the asymmetry in the lateral loading while one wingtip flies past a wall may affect the roll stability of the aircraft.

\subsection{Reynolds Number and Vortices}

When studying wingtip vortices, it is important to recognize the importance of the Reynolds number of the aircraft under consideration. In a paper by Delisi and Greene [17] the migration and persistence of a vortex pair in a nonstratified fluid is investigated. Laboratory tow tank tests are completed with airfoils with chord Reynolds numbers from 
40,600 to 124,200 . The authors arrive at the intuitive result that high Reynolds number vortices migrate farther and last longer than low Reynolds number vortices [17].

\subsubsection{Low Reynolds Number and Vortices}

In a paper by Mueller [18], the low Reynolds numbers of fixed wing MAVs are discussed and a plot shows that these vehicles operate with Reynolds numbers in the range of 10,000 to a little over 100,000. This range of Reynolds numbers is considered to be very low since a full size Cessna 210 and a Boeing 747 have Reynolds numbers of about $1,000,000$ and 10,000,000 respectively [18].

Wingtip vortices are studied for a rectangular, delta, and semi-elliptical wing with low aspect ratios and low Reynolds numbers in a paper by Kaplan et al [19]. Although PIV testing in a water tunnel shows variations in the wingtip wake structures, the lift curve slope $(\sim \pi)$ is not affected by the different planform shapes or Reynolds numbers. The PIV tests yield the best images of the wingtip vortex for the rectangular wing because these vortices have the tightest roll-up of vorticity and the highest magnitudes of vorticity when compared to the other wing planform shapes. However, the wingtip vortices from the different wings did give similar results for maximum circulation [19].

In a paper by Zuhal and Gharib [20], wind tunnel tests are completed in order to study the wingtip vortices of a NACA 0012 airfoil with a low Reynolds number of 9040 based on chord length. Stereoscopic Particle Image Velocimetry (SPIV) tests are completed in order to visualize the wingtip vortices and it is discovered that the main vortices are surrounded by satellite vortices. These satellite vortices introduce their own velocity field and cause the wingtip vortex to fluctuate in its position [20]. Kaplan et al 
[19] also discuss this same paper and theorize that the Reynolds number would have an effect on the strength of the satellite vortices.

\subsubsection{High Reynolds Number and Vortices}

Chow et al [12] discuss wind tunnel testing used to study the initial near field rollup of a turbulent wingtip vortex specifically relating to a wing with a high Reynolds number based on chord of $4,600,000$. The results show that an advantageous axial pressure gradient is located in the core of the wingtip vortex, which is a result of the crossflow velocities of the tip vortex. This pressure gradient causes the maximum axial velocity of the vortex to have a value of 1.77 times the freestream velocity and a location just upstream of the trailing edge of the wing. The high Reynolds number is one of the contributing factors to why the core axial velocity is higher for this wingtip vortex compared to what the authors have seen in the past [12].

\subsubsection{Reynolds Number Sensitivity for Free Vortex Propagation}

While the two previous sections have specifically described research that focuses on only high or low Reynolds numbers and vortices, additional research has been devoted to bridging the gap between these two disciplines. In a paper by Delisi [21], turbulence measurements for the wakes of full-scale aircraft as well as a model wing in a water tunnel are investigated. A C-130, B757, and B767, and the laboratory small, thin cambered plate are used in the analysis. The Reynolds numbers of the laboratory plate and two full size aircraft can be seen in Table 2.1. Figure 2.8 shows the values of the normalized turbulence from the wake of all the aircraft used in the analysis. It is 
significant that the normalized values of the turbulent dissipation rate between the vortex cores are approximately equal across all of the full size aircraft and the laboratory plate. This means that there is a lack of Reynolds number sensitivity for the free vortex propagation. Since this data is very similar, it also shows that the wake is created by the wings and fuselage, and that the wake is not dependent on the various types of propulsion systems that these aircraft use [21].

Table 2.1: Chord and vortex Reynolds numbers for the laboratory experiment and full size aircraft [21].

\begin{tabular}{|l|c|c|}
\hline & $\begin{array}{c}\text { Chord Reynolds } \\
\text { Number, } \operatorname{Re}_{\mathrm{c}}\end{array}$ & $\begin{array}{c}\text { Vortex Reynolds } \\
\text { Number, } \mathbf{R}_{\mathrm{v}}\end{array}$ \\
\hline NWRA lab experiments & 64,000 & 32,000 \\
\hline A320 in cruise & $22,000,000$ & $7,000,000$ \\
\hline B747 in cruise & $55,000,000$ & $15,000,000$ \\
\hline
\end{tabular}

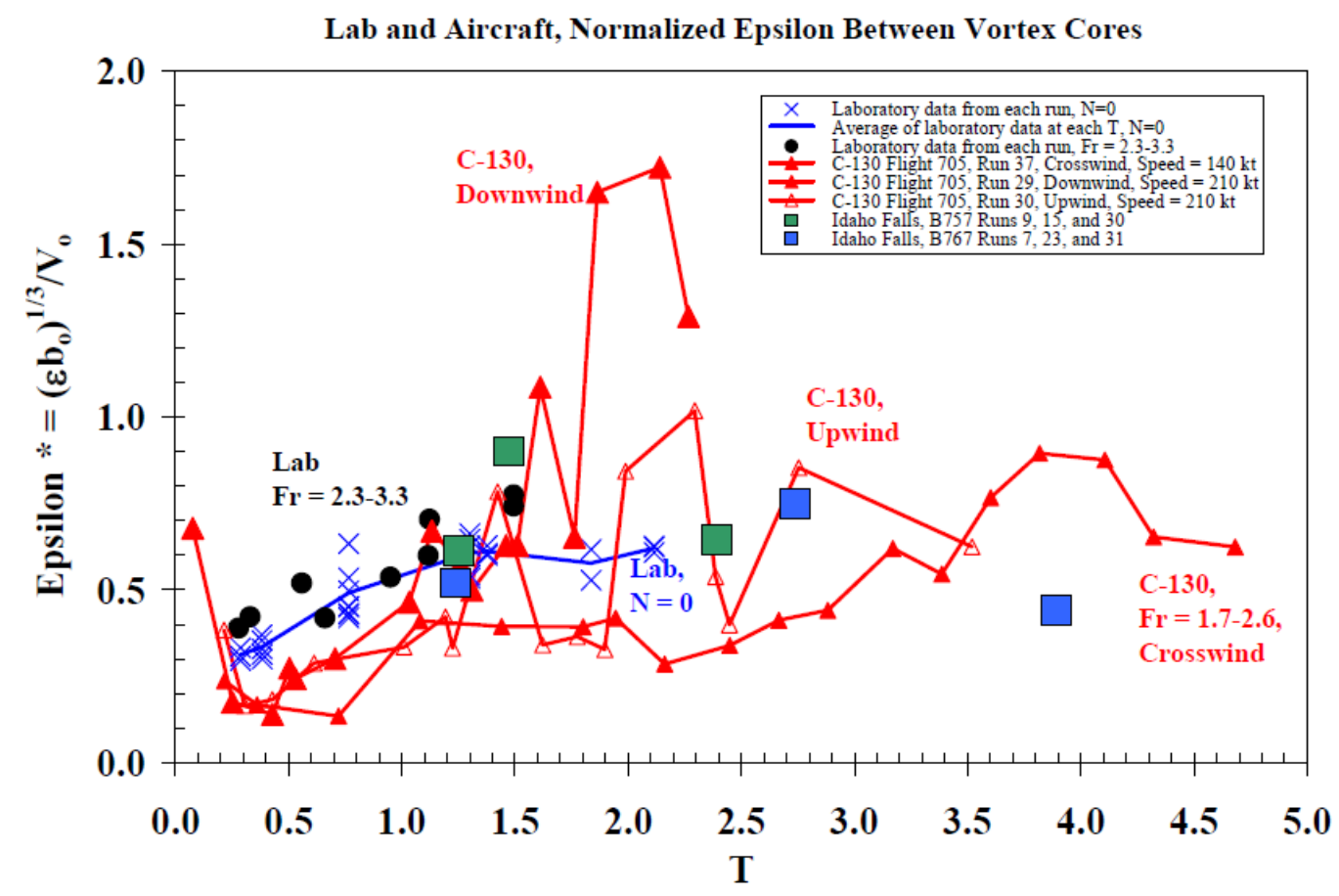

Figure 2.8: Normalized turbulence, $\epsilon^{*}$, between the vortex cores for the C-130, B757, B767, and laboratory plate [21].

This research relates to the wall effects study in this thesis because it helps to justify using two different wings with slightly different Reynolds numbers for the wall 
effects wind tunnel testing performed for this thesis. The lack of Reynolds number sensitivity involved in free vortex propagation justifies this decision and will be discussed in more detail later in this thesis.

\subsection{Vortex Wall Interaction}

In a paper by Koumoutsakos [22], vortex wall bounded flows are investigated. A vortex flow is recognized along a wall through measuring the wall pressure and calculating the resulting vorticity flux. An uncontrolled vortex dipole is investigated and Figure 2.9 shows contour plots of the vorticity field for the vortex as it approaches the wall along the bottom axis. This vortex interacts with the wall to create a weaker secondary vorticity of the opposite sign at the wall surface [22]. This research relates to the wall effects research discussed in this thesis since the wall will have a direct impact on the vortex's deformation/reformation.
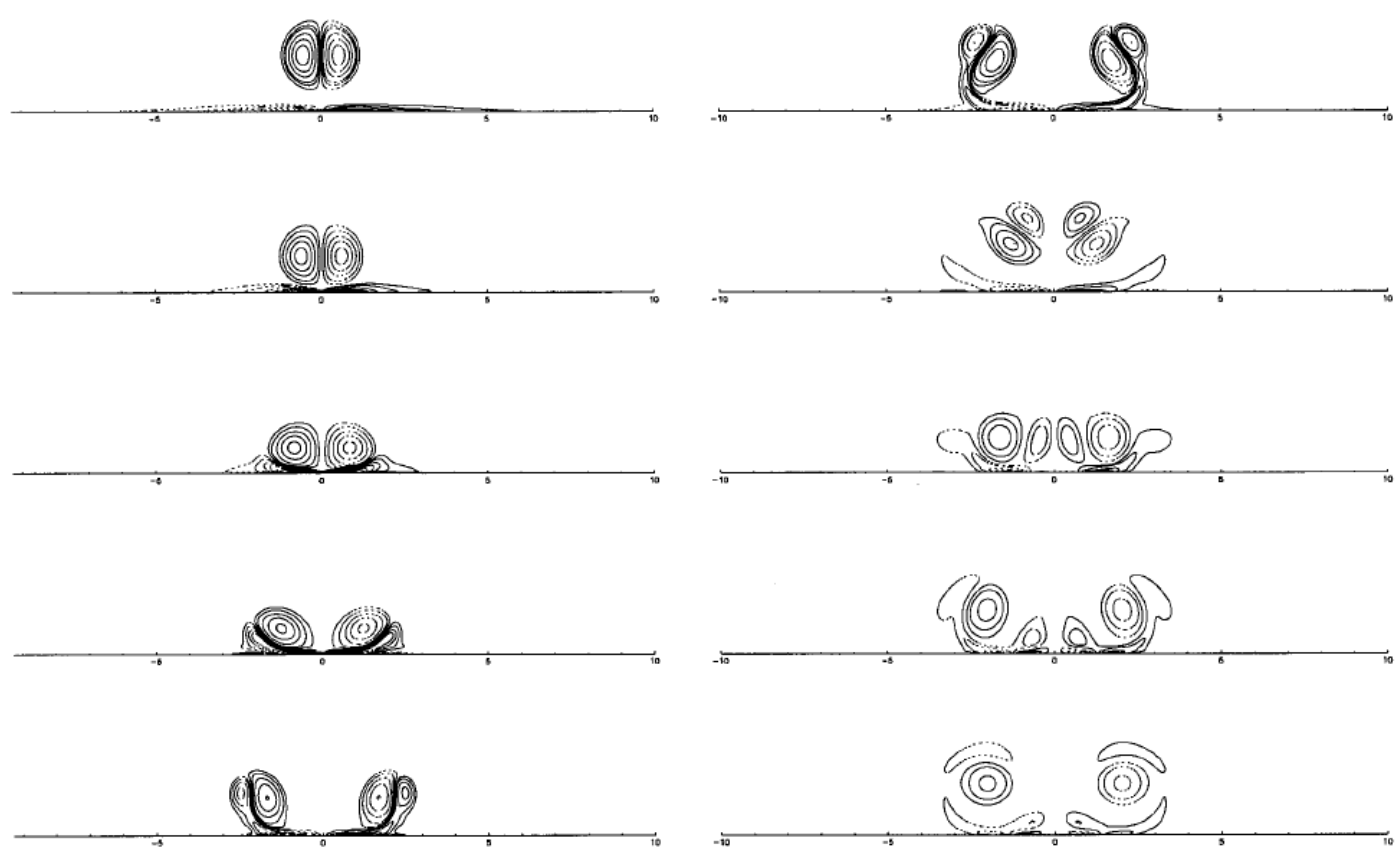

Figure 2.9: Vortex dipole interactions with a wall without any control parameters [22]. 
A vortex particle-in-cell method is developed by Ho Liu and Doorly [23] in order to analyze a vortex dipole impacting a wall at normal and oblique collision angles. For a perpendicular vortex dipole collision with a wall, this method produces results similar to the work described earlier by Koumoutsakos [22] when no control parameters are used as shown in Figure 2.9. However, Figure 2.10 shows a contour plot of the vorticity of the vortex dipole as it impacts the wall at a $75^{\circ}$ angle. This vortex dipole impact is different from the perpendicular impact because as time increases down the column of images, the secondary vortex, of the left primary vortex, is absorbed into the right primary vortex. In the perpendicular vortex dipole impact, the secondary vortices for each primary vortex absorb back into their corresponding primary vortices. Figure 2.11 shows a contour plot of the vorticity of the vortex dipole as it impacts the wall at a $45^{\circ}$ angle. This vortex dipole impact is different from the perpendicular and $75^{\circ}$ impact because as time increases as one looks down the column of images, the left primary vortex is constricted by the right primary vortex. When the left primary vortex meets the wall, it generates vorticity of opposite sign and is eventually swept around the right primary vortex [23]. This research is relevant to the wall effects research in this thesis because when the aircraft flies along a wall and performs a banked turning maneuver, the wingtip vortex may not impact the wall at a perpendicular angle. 

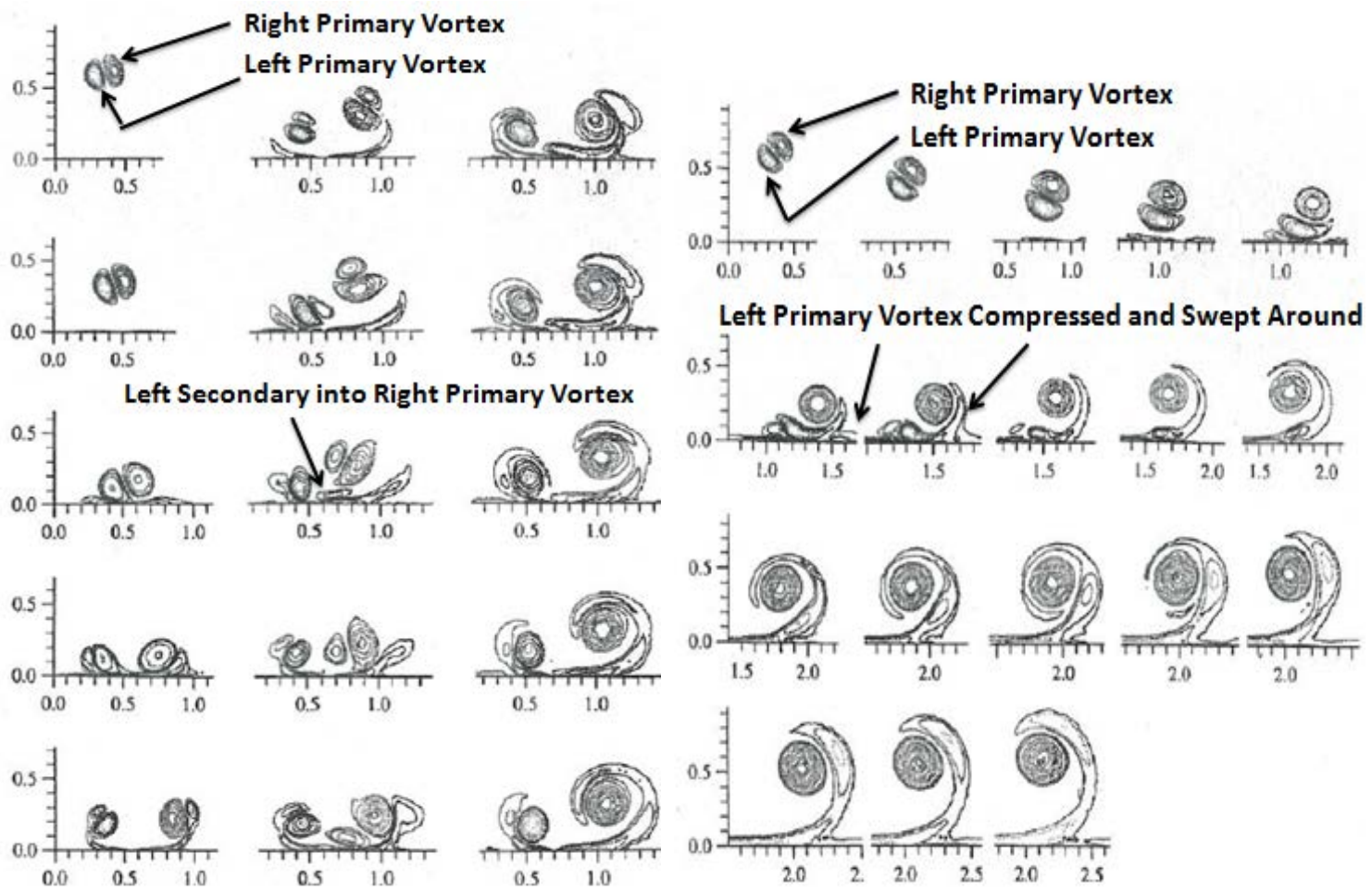

Figure 2.10: Contour plot of the vorticity of the vortex dipole as it impacts the wall at a $75^{\circ}$ angle [23].

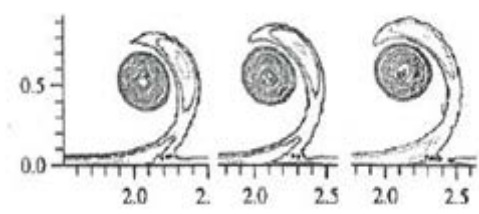

Figure 2.11: Contour plot of the vorticity of the vortex dipole as it impacts the wall at a $45^{\circ}$ angle [23].

\subsection{Vortex Ellipticity}

A paper by Le Dizes and Laporte [24] discusses the elliptical instability present when two vortices are located in close proximity to one another. This elliptical instability affects strain and merging processes between the two vortices. "This 'elliptical' instability, which is characterized by a three-dimensional deformation of the vortex cores, has been found for both counter-rotating and co-rotating vortices" [24]. This statement demonstrates the significance of the elliptical instability because it is a three-dimensional instability and is strong enough to deform the streamlines around a vortex core into an elliptical shape. The authors go on to discuss the application of this type of vortex instability present between co-rotating vortices from a wingtip and a flap on the wing. 
These two vortices experience the elliptical instability which has been shown to be a convective instability [24].

The three-dimensional destabilization of vortices is investigated in a paper by Potylistsin and Peltier [25]. The goal of this paper is to apply various amounts of ellipticity to Stuart vortex models in order to study the linear stability of these twodimensional column vortices and determine the dominant three-dimensional instabilities present in the vortices. The Stuart vortex model is chosen for this research since it is easy to modify the ellipticity value for this type of vortex model. Ellipticity is measured by the vortex parameter, $\rho_{\mathrm{v}}$, and values of $0.33,0.50$ and 0.75 are used for this variable. As this variable increases in value, the ellipticity of the Stuart vortex actually decreases.

Figure 2.12 shows each of these elliptical Stuart vortices. [25].

- $\rho_{\mathrm{v}}=0.33$ highly elliptical vortex

- $\rho_{\mathrm{v}}=0.50$ moderately elliptical vortex

- $\rho_{\mathrm{v}}=0.75$ vortex with low ellipticity

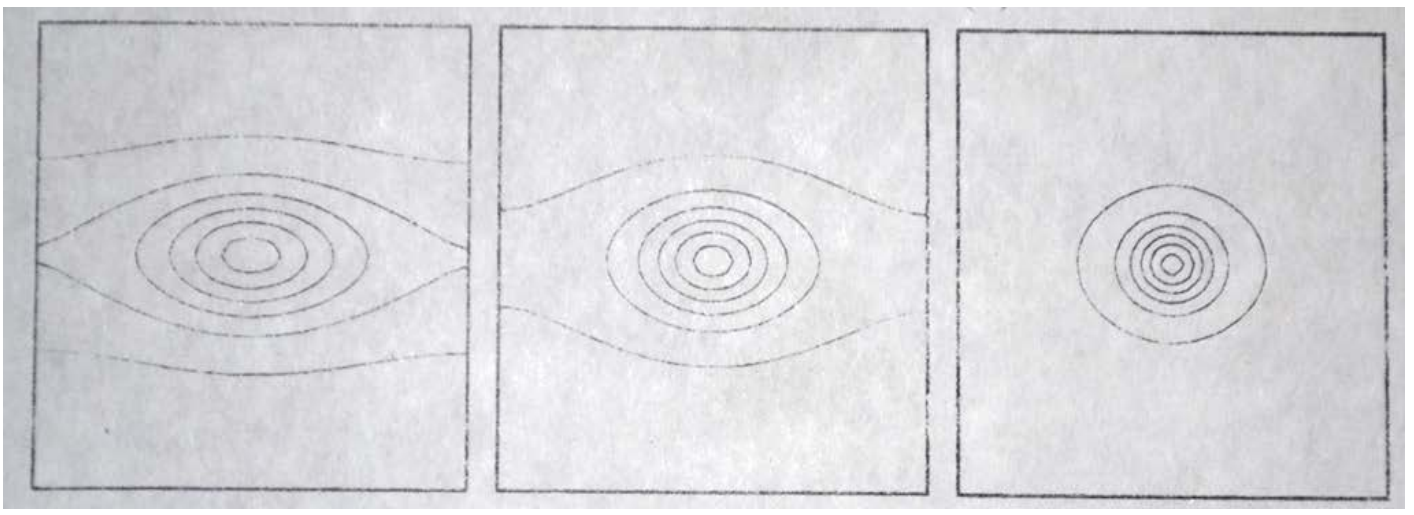

Figure 2.12: Left to right: high, moderate, and low ellipticity for ideal two-dimensional vorticity Stuart model vortices [25]. 
The three different three-dimensional instabilities that are analyzed within this research include the following.

- Elliptical Instability- elliptical shaped instability affected by the Coriolis force. The Coriolis force relates to the deflection of an object's path with reference to a moving coordinate system.

- Edge Instability- a centrifugal instability on the edge of a columnar vortex.

- Hyperbolic Instability- an instability that relates to the stagnation point between two vortices in close proximity to one another [25].

The results of this research show that for a highly elliptical Stuart vortex, the elliptical and hyperbolic instabilities are the three-dimensional instabilities that dominate this vortex. This elliptical instability is found to be located in the core of the vortex for low spanwise wavenumber vortices while the hyperbolic instability is found in the stagnation point between two vortices if the wave number is high. A moderately elliptical Stuart vortex is dominated by the elliptical three-dimensional instability located in the core of the vortex. However, a less dominant edge instability also exists for this moderately elliptical vortex and it is located on a ring around the edge of the vortex. A Stuart vortex with low ellipticity is dominated by the edge instability on the edge of the vortex. However, the elliptical instability also remains in the core of the vortex but is not as strong as the edge instability. Figure 2.13 shows examples of the spanwise vorticity fields for the highly elliptic, moderately elliptic, and low vortex ellipticity Stuart vortices. The dashed lines represent isolines with negative values while the solid lines represent 
isolines with positive values. The areas of dominant instability can be seen in each test case as described above [25].
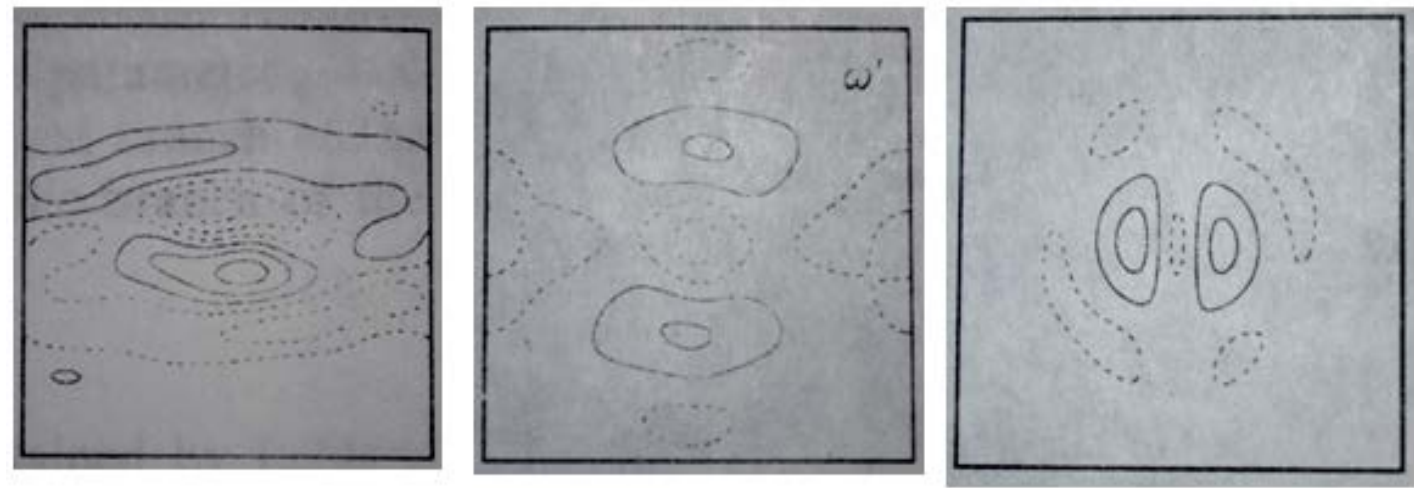

Figure 2.13: Left to right: high, moderate and low ellipticity spanwise vorticity fields showing the instabilities present in each type of vortex [25].

\subsection{Numerical Methods of Simulation}

Numerical simulations of vortex wakes are also discussed by Ginevsky and Zhelannikov [10]. The advantages and disadvantages of direct numerical simulation, large eddy simulation, and Reynolds-averaged Navier-Stokes methods are discussed, but it is concluded that the discrete vortex method (DVM) is the simplest and most effective method to use when studying aircraft wakes with high Reynolds numbers. Ginevsky and Zhelannikov also discuss the DVM during takeoff and landing vortex wakes since ground effect is present [10].

In a paper by Orlandi [26], a numerical method is developed to simulate vortex dipoles interacting with a wall. The Navier Stokes equations are discretized and energy and entropy are conserved. A centered, $2^{\text {nd }}$ order discretization method is applied to find the viscous terms and the nonlinear terms are found through using an explicit third-order Runge-Kutta method discretized in time. The results from this numerical simulation 
agree with experimental results that the authors cite. The advantage of this numerical simulation lies in the fact that it can investigate how a vortex dipole interacts with a wall for a longer period of nondimensional time compared to experiments. "The longer time evolution of the present simulation revealed very interesting features, such as multiple rebounding and pairing of vortices forming new dipoles that detach from the wall" [26].

Figure 2.14 demonstrates these characteristics of the vortex dipole interaction with a wall.

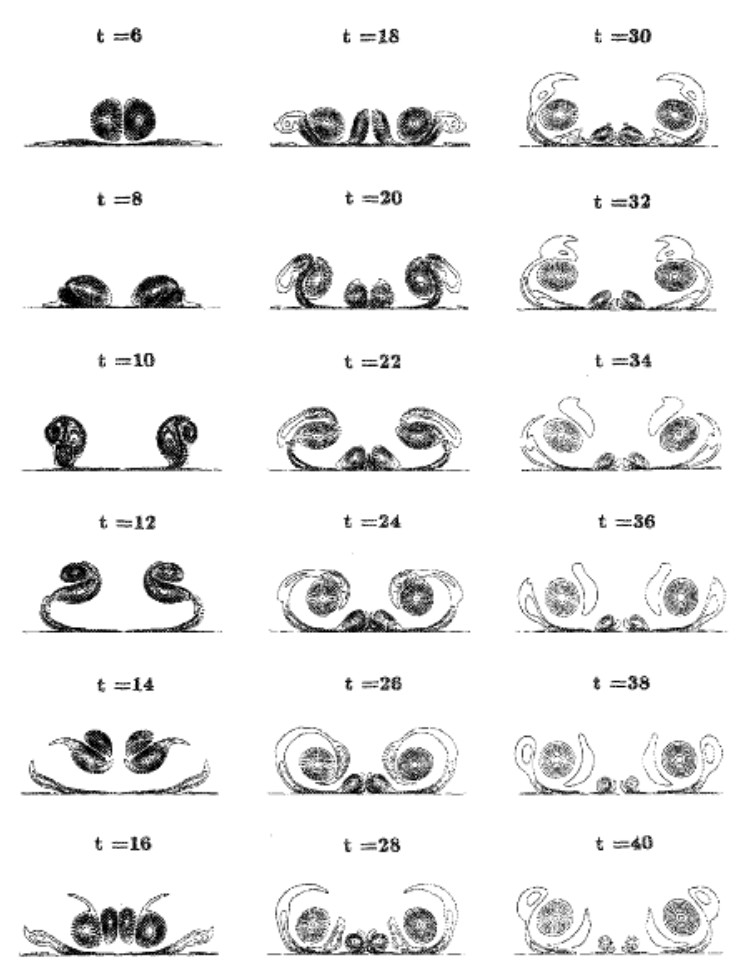

Figure 2.14: Contour plots of the vorticity of the vortex dipole as it interacts with a wall over nondimensional time [26].

\subsection{Wind Tunnel Testing}

Just as numerical methods can be used to analyze how a vortex interacts with a wall, wind/water tunnel testing can also be used as a form of validation. Igarashi et al [27] describe wind tunnel testing that investigates how a wingtip vortex is affected by the 
presence of a wall. A wall is placed in the closed-circuit low-speed wind tunnel at Iowa State University in order to analyze the behavior of a wingtip vortex structure in close proximity to a wall for a square-tipped, rectangular NACA0012 wing. The cross sectional dimensions of the wind tunnel, size of the wing, direction of air flow, and the position of the wing can be seen in Figure 2.15a. The wall-to-tip distance ranges from six, four, two, and one inch as shown by the dimension "a" in Figure 2.15b. A velocity vector field showing the position of the flow of an example wingtip vortex is also seen in Figure 2.15b [27].

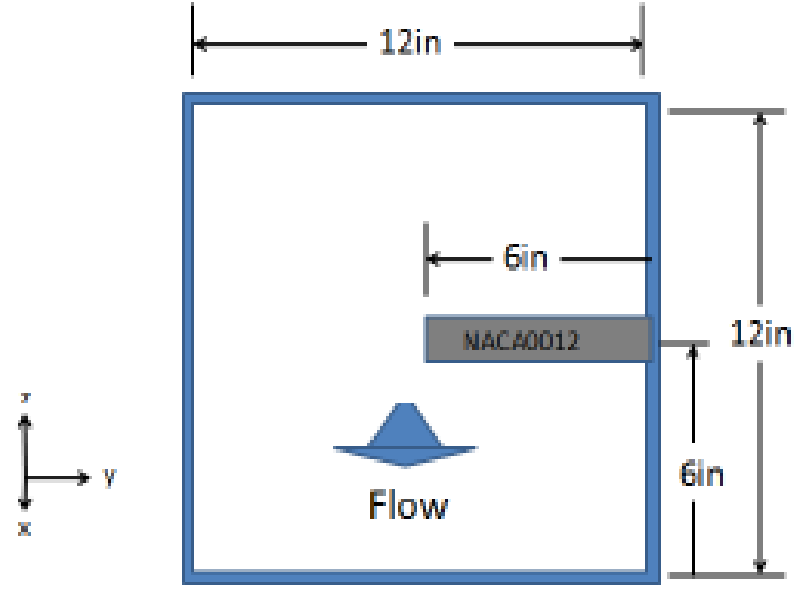

Figure 2.15a: Dimensions of the cross section of the wind tunnel test section with the wall insert [27].

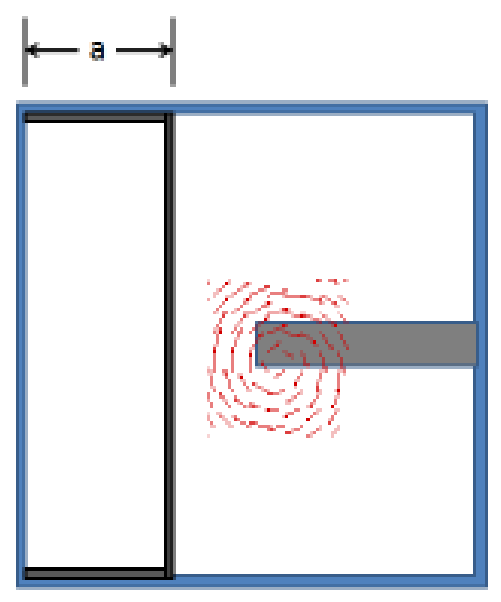

Figure 2.15b: Tunnel cross section with wall insert. Dimension "a" ranging from $1,2,4$, and 5 in [27].

Figure 2.16 shows the average location of the wingtip vortex with different tip-towall distances measured as decimal values multiplied times the chord of the wing (c). As the inserted wall moves closer to the wingtip, the vortex core begins to rise in elevation. When the wall is removed from the test section, the $\mathrm{z} / \mathrm{c}$ value is -0.052 , which is very close to the wing itself. The wall can be understood as an imaginary mirror vortex to the 
actual wingtip vortex. This imaginary vortex of opposite rotation causes the vortex upwash to drive the wingtip vortex upward as the wall gets closer to the wingtip [27].

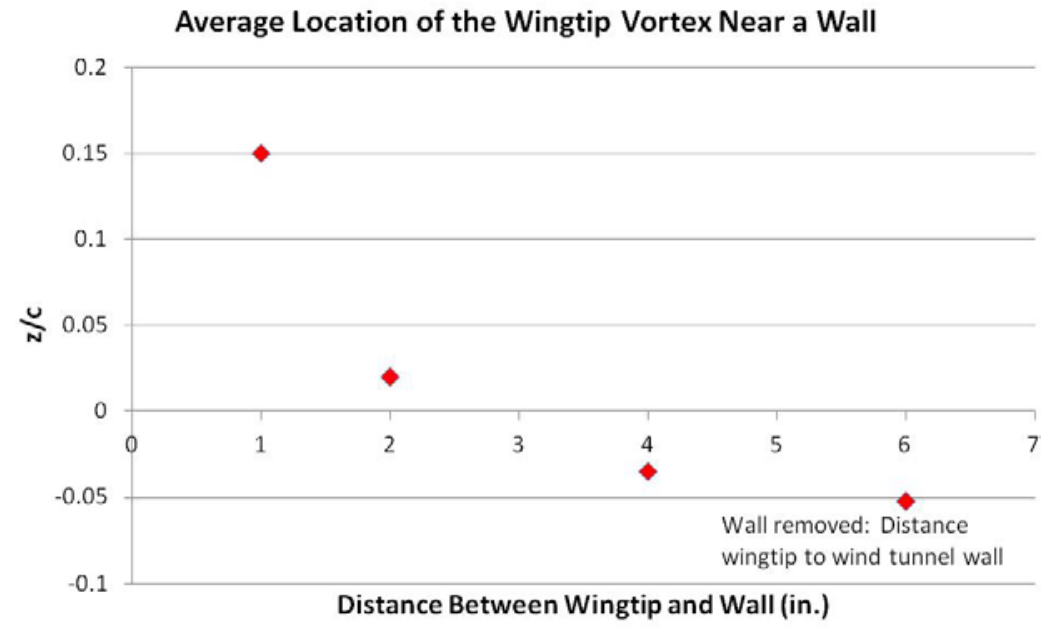

Figure 2.16: The average location of the wingtip vortex core with varying tip-to-wall distances [27].

Figure 2.17 shows a log-log scale plot of the wall-to-tip distance and the up-wash driven height of the vortex. The equation of the line is $\ln y=-1.69 \log x+8.61$.

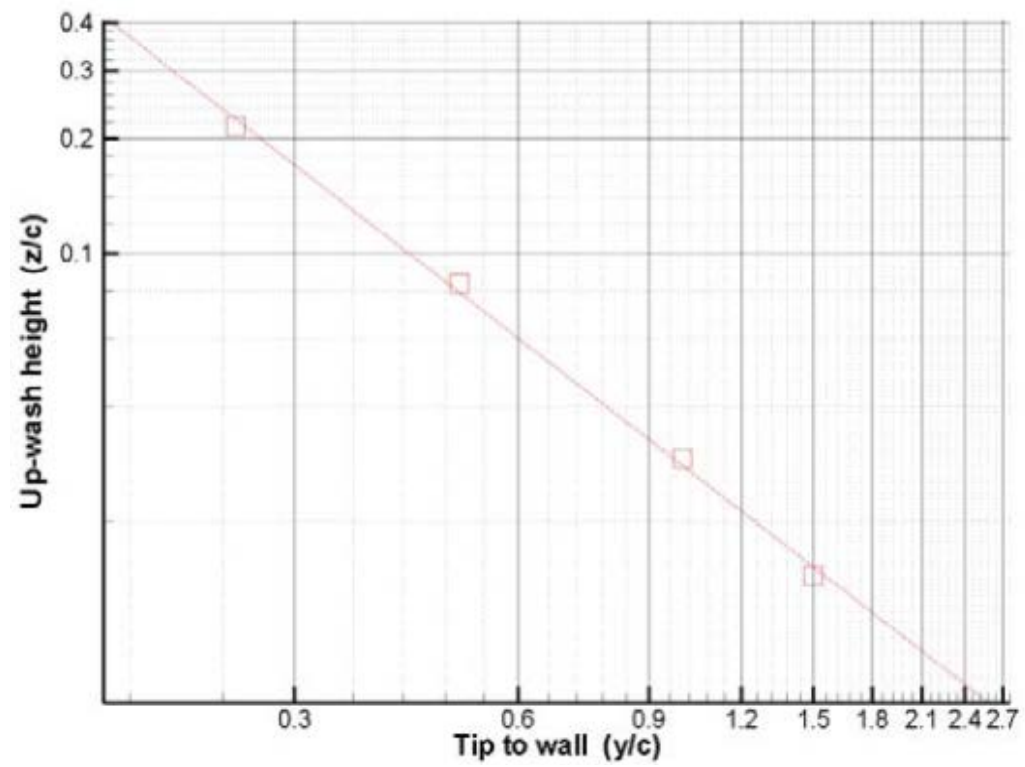

Figure 2.17: Distance from wall to wingtip vs. the mean vortex core elevation. Alpha $=5^{0}$ and $\mathrm{x}=4 \mathrm{c}$ [27]. 
Figure 2.18 shows the various vortex core radius sizes as well as different vortex vorticity values at different distances away from the wall. The vortex radius grows as it gets closer to the wall until it is about one inch away from the wall, at which point it gets smaller due to the fact that it is less than five vortex core radii away from the wall. The vorticity of the vortex is not affected until it is located within five vortex core radii away from the wall, at which point the magnitude of the vorticity decreases by about $10 \%$ [27].

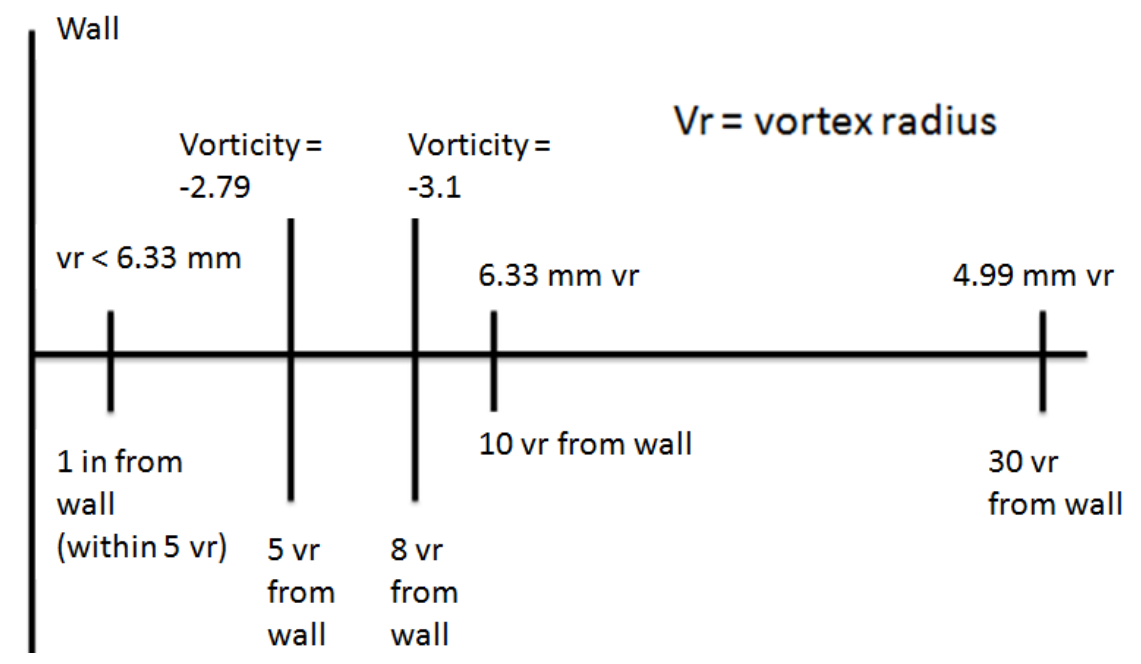

Figure 2.18: Various vortex core radius sizes as well as different vortex vorticity values at different distances away from the wall.

Overall, this paper shows that as the wall moves closer to the wingtip vortex, the vortex radius grows and the vortex location changes. The wall can be thought of as an imaginary mirror vortex to the wingtip vortex. This mirror vortex grows in size and causes the vortex up-wash to drive the vortex upward in elevation.

All of this research by Igarashi et al [27] relates to the wingtip vortex wind tunnel wall effects research in this thesis since the experimental setup is very similar. It is expected that similar results will be obtained and that the vortex up-wash will drive the vortex upward as the wall gets closer to the wingtip. This vortex up-wash could 
contribute to higher $\mathrm{C}_{\mathrm{L}}$ values on the aircraft as the wall approaches the wingtip. The research by Igarashi et al also relates back to the ground effect explanation by Barnes [15] earlier in this thesis.

A paper by Torres and Mueller [28] discusses low-aspect-ratio (AR below two) wing aerodynamics at low Reynolds numbers (between 70,000 and 200,000). In this research, the wing planform, aspect ratio, and Reynolds numbers effects are investigated by varying all of these parameters across rectangular, Zimmerman, inverse Zimmerman and elliptical planforms. Then, all of the wing samples are tested in the low-turbulence, indraft, open-circuit wind tunnel at the University of Notre Dame. The lift, drag, normal force, and pitching moment are all measured for the various wings tested. A transition point is discovered for the $C_{\text {Lmax }}$ Vs. AR curve (Figure 2.19) and the $\alpha_{C L m a x}$ Vs. AR curve (Figure 2.20). This transition is seen by the sudden drop in the $\mathrm{C}_{\mathrm{Lmax}}$ and $\alpha_{\mathrm{CLmax}}$ values, indicating a transition from nonlinear to linear lift generation mechanisms at high angles of attack near stall. Since the Night Vapor RC airplane utilized in this research has an aspect ratio of 2.74 , which is barely off of these plots, it is expected that this aircraft will have a $\mathrm{C}_{\mathrm{Lmax}}$ value in the range of 0.65 to 0.8 . It is also expected that the Night Vapor will have a $\alpha_{\text {CLmax }}$ value of around $13^{0}$ to $17^{0}$ since the rectangular and elliptical planforms used by Torres and Mueller [28] are closest to the planform shape of the Night Vapor. Based on the aspect ratio of the Night Vapor, it is expected to have linear lift generation mechanisms at high angles of attack near stall, since this is the trend for planforms with aspect ratios from around 1.75 and higher [28]. 


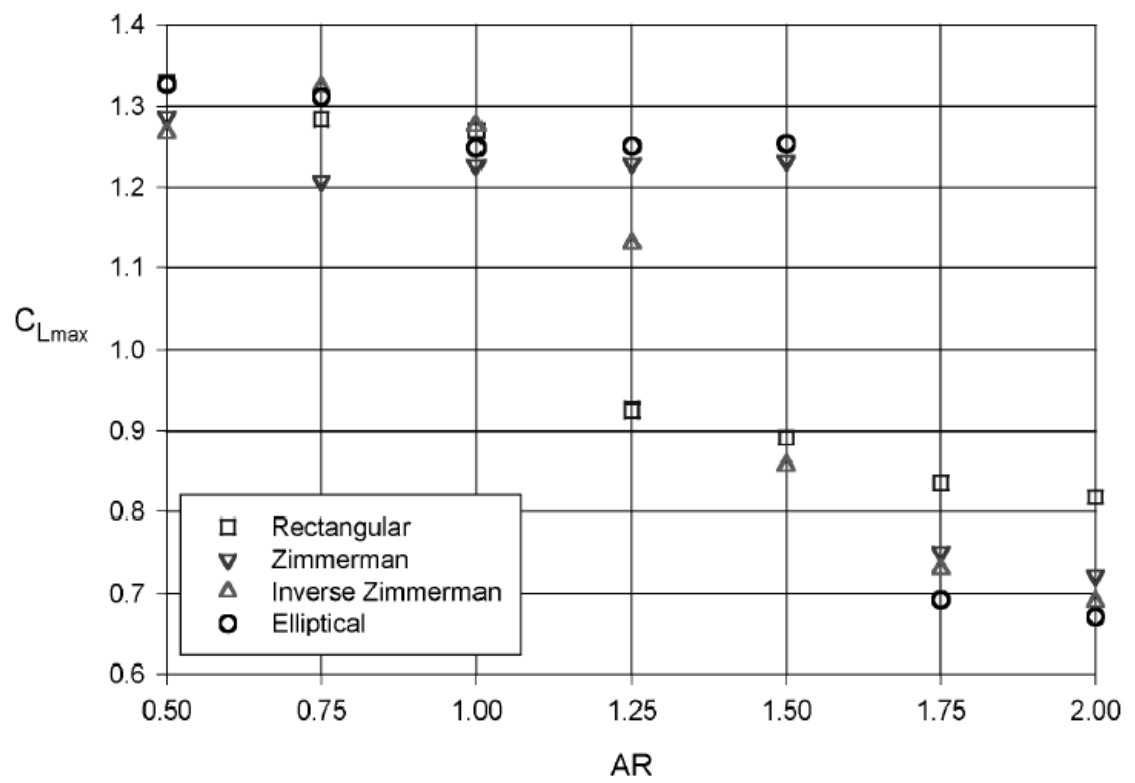

Figure 2.19: $\mathrm{C}_{\mathrm{Lmax}}$ and aspect ratio values for four wing planforms [28].

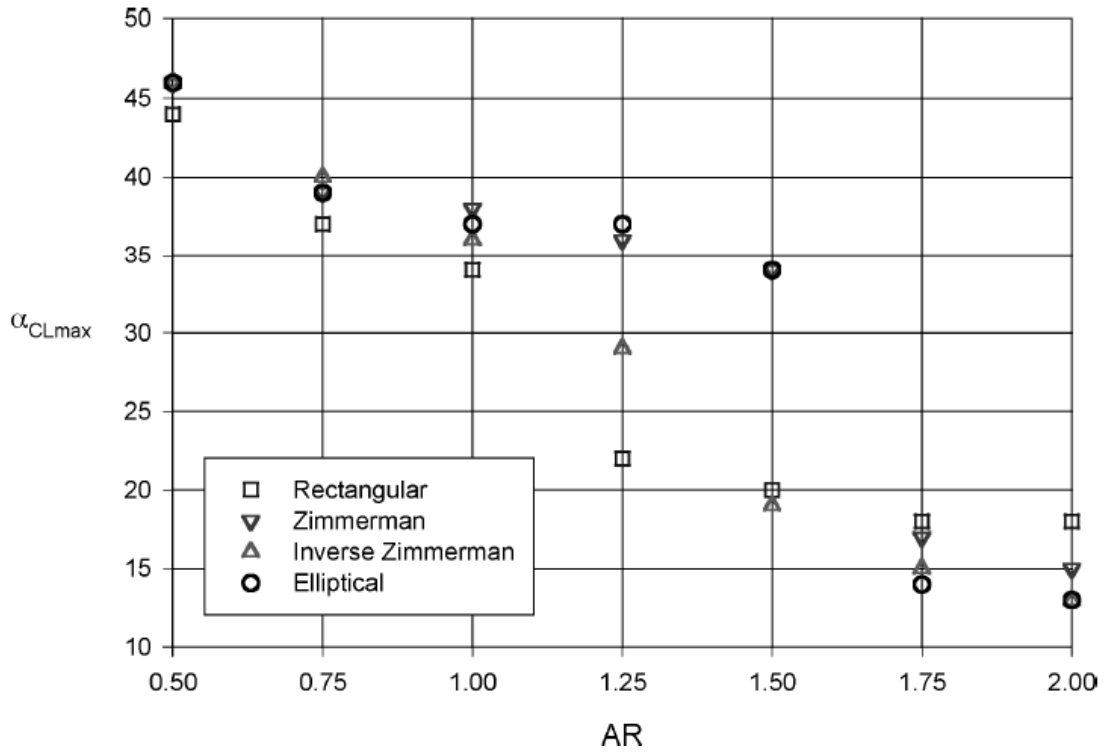

Figure 2.20: $\alpha_{\text {CLmax }}$ and aspect ratios for the four wing planforms tested [28].

\subsection{Free Flight Vortex Wake Experiment for a MAV Sized Bird}

While much research studies the wake characteristics of wings or cylinders near walls through the use of numerical simulations or wind tunnel experiments, little research exists into measuring free flight MAV wake flows. However, the wake characteristics of 
a live gliding kestrel bird are investigated in a paper by Spedding [29]. According to the author, this research from 1987 is the first time a free flying animal has been used to analyze flight performance characteristics from the wake of the animal's wing. Figure 2.21 shows a drawing of the test setup in a hallway. The velocity of the kestrel is calculated by using a stopwatch to measure the amount of time it took the bird to fly the distance between its starting and ending positions [29].

Figure 2.22 shows a representative wingtip vortex captured from one of the tests. The corkscrew shape of the wingtip vortex tube can clearly be seen above the number three in the image [29].

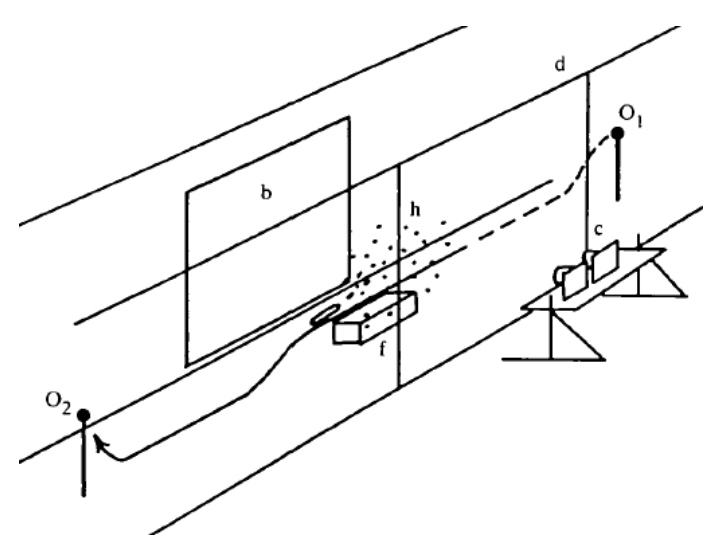

Figure 2.21: Test setup for wake measurements from a kestrel gliding through a hallway [29].

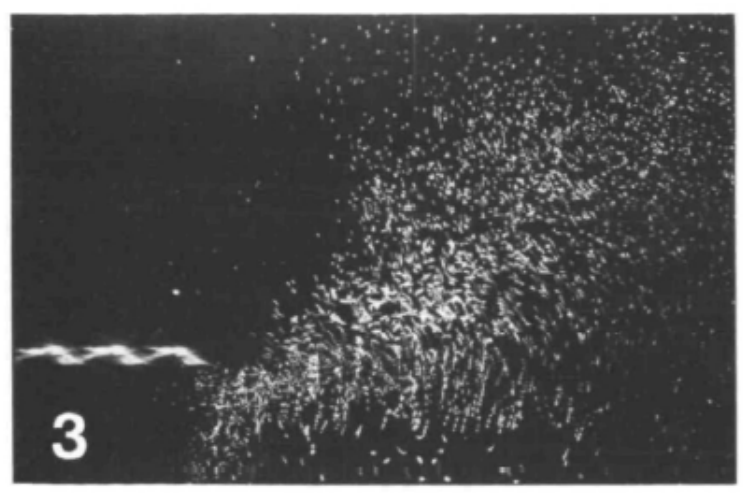

Figure 2.22: Corkscrew shape of a wingtip vortex can clearly be seen [29].

The results of Spedding's research [29] show that the wake of a free flying kestrel can accurately be described by classical aircraft theory. It is also concluded that the steady state lifting line analysis can be applied to an elliptically loaded wing pair and still provide accurate calculations of the induced velocities in the wake. Overall, this research shows that it would be possible to use the data collected in this research to build an accurate generic model of a live bird's flight [29]. 
Spedding's research [29] is unique in the fact that the wake of a MAV sized bird is analyzed in free flight tests. This test setup and procedure for this research is similar to the testing procedure and setup for the free flight MAV wingtip vortex wall effects research described in this thesis. 


\section{CHAPTER 3}

\section{THEORETICAL BACKGROUND}

\subsection{Theory behind Experiment}

The research in this thesis is based on the theory that as a MAV flies along a wall, the MAV's wingtip vortex nearest the wall deforms due to the vortex's proximity to the wall. The goal is to then determine whether the deformation of this vortex affects the handling of the aircraft while it flies in close proximity to the wall. Any vortex has an influence on a flow or body located in its surrounding environment. The degree of influence that a vortex has on the flow or body in its environment is a direct function of the distance between the vortex and the body or flow.

Through using Particle Image Velocimetry (PIV) testing techniques, it is possible to collect data for the wingtip vortex wake of a MAV freely flying along a wall in an indoor flight test facility. Analyzing the wingtip vortex wake helps to tell the story about what happens to the wingtip vortex lift force acting on the wing as a result of the vortex's interaction with a wall. The circulation of the wingtip vortex can be calculated through processing PIV images and this helps to calculate the vortex lift force acting on the wing. Measuring the ellipticity of the wingtip vortex wake also provides insight into the deformation of the wingtip vortex due to instabilities that exist from the vortex's 
proximity to the wall. Determining the location of these instabilities within the wingtip vortex wake can also help to examine how these instabilities affect aircraft handling while flying in close proximity to a wall.

While analyzing the wake provides one frame of reference for measuring the aerodynamic effects involved with an aircraft flying in close proximity to a wall, wind tunnel testing can also be used. This frame of reference makes it possible to measure the amount of lift force acting directly on the aircraft while running a wind tunnel with and without a wall placed in close proximity to the wingtip of an aircraft mounted on a force balance. This makes it possible to calculate the coefficient of lift for the aircraft.

A numerical approach to modeling the behavior between a vortex and a wall is also investigated in an attempt to validate the results seen in the PIV wingtip vortex deformation and wind tunnel lift force results. However, the potential flow model attempted in this thesis research did not yield results concerning how the wingtip vortex interaction with a wall affects MAV flight controls. Therefore, the details of the potential flow model are contained in Appendix B and will not be discussed throughout the remainder of the primary text of this thesis.

A total of eight different types of tests are executed for the free flight and wind tunnel tests in order to analyze the effect of the wall on the aircraft during typical MAV flight conditions. The test matrix can be seen in Table 3.1. The four tests listed in Table 3.1 will be repeated without a wall present. These tests without the wall serve as a baseline for comparison against the tests performed with the wall present. 
Table 3.1: Test matrix for free flight and wind tunnel tests.

\begin{tabular}{|c|c|c|c|}
\hline \multicolumn{4}{|c|}{ Test Matrix } \\
\hline Test Number & Speed & Orientation of Aircraft & Wall or No Wall \\
\hline 1 & Fast & Straight and Level & Wall \\
\hline 2 & Slow & Straight and Level & Wall \\
\hline 3 & Fast & Turning Maneuver & Wall \\
\hline 4 & Slow & Turning Maneuver & Wall \\
\hline
\end{tabular}

The lift force acting on an aircraft during a banked turn will be higher than the lift required for the same aircraft at the same velocity flying straight and level. This should be evident in the wind tunnel tests from the airplane's frame of reference. If the lift is higher for the banked turning maneuver tests, the circulation of the wingtip vortex should also be higher for the banked turning flights compared to the straight and level flights at the same flight velocity. The two different flight velocities tested will also have an effect on the aircraft's wingtip vortex circulation value. The Kutta-Joukowsi theorem is explained in detail in section 3.4 and this theorem shows that if the aircraft flies at a slower speed and still needs to support the same air vehicle weight, then the circulation must increase proportionally to the decrease in velocity. This assumes that the lift of the aircraft is equal to the weight.

\subsection{Vortex Identification Methods}

In order to accurately identify and perform calculations on a vortex in an experimental flow, it is first necessary to have a fundamental understanding of the definition of a vortex. Robinson [30] provides the following definition.

A vortex exists when instantaneous streamlines mapped onto a plane normal to the vortex core exhibit a roughly circular or spiral pattern, when viewed from a reference frame moving with the center of the vortex core [30]. 
While this definition may indicate that identifying a vortex is a straightforward process, a precise approach must be taken in order to accurately and consistently pinpoint the core and the boundaries of a vortex when the vortex is not clearly defined. In a paper by Graftieaux et al [31] vortex identification functions are developed to systematically identify the center of the vortex core and the boundary of a vortex derived from PIV measured velocity fields. The center of the vortex is pinpointed through calculating the dimensionless scalar $\Gamma_{1}$ in Equation 3.1.

$$
\Gamma_{1}(P)=\frac{1}{N} \sum_{S} \frac{\left(P M^{\wedge} U_{M}\right) \cdot z}{\|P M\| \cdot\left\|U_{M}\right\|}=\frac{1}{N} \sum_{S} \sin \left(\theta_{M}\right)
$$

$\Gamma_{1}(P)=$ a dimensionless scalar used to pinpoint the vortex core center.

$\mathrm{P}=$ the location where $\Gamma_{1}(P)$ is calculated.

$\mathrm{S}=$ the two dimensional area surrounding point $\mathrm{P}$.

$\mathrm{U}_{\mathrm{M}}=$ the velocity vector around the vortex.

$M=$ the point surrounding point $P$ where the velocity vector $U_{M}$ is located.

$\mathrm{PM}=$ radius vector from point $\mathrm{P}$ to point $\mathrm{M}$.

$\mathrm{Z}=$ the unit vector normal to the measurement plane.

$\theta_{M}=$ the angle between the velocity vector $\mathrm{U}_{\mathrm{M}}$ and the radius vector PM.

$\mathrm{N}=$ the number of points $\mathrm{M}$ inside of $\mathrm{S}$.

$\wedge=$ this symbol represents an exterior product, which is a cross product of $\mathrm{PM}$ and $\mathrm{U}_{\mathrm{M}}$.

A paper by Morgan et al [32] utilizes the $\Gamma_{1}$ vortex core identification method and provides a thorough explanation and diagram showing the theory behind this method.

Figure 3.1 shows the location of the variables used in the $\Gamma_{1}$ vortex core identification method. The velocity vectors are from PIV measurements and are represented by the red arrows circulating point $\mathrm{P}$. The point $\mathrm{M}$ represents the base of the vector under consideration. Every vector surrounding point $\mathrm{P}$ has a corresponding PM radius vector. Every $\mathrm{PM}$ radius vector is used in the sum in Equation 3.1 to determine if that point $\mathrm{P}$ is indeed a point in the core of the vortex [32]. 


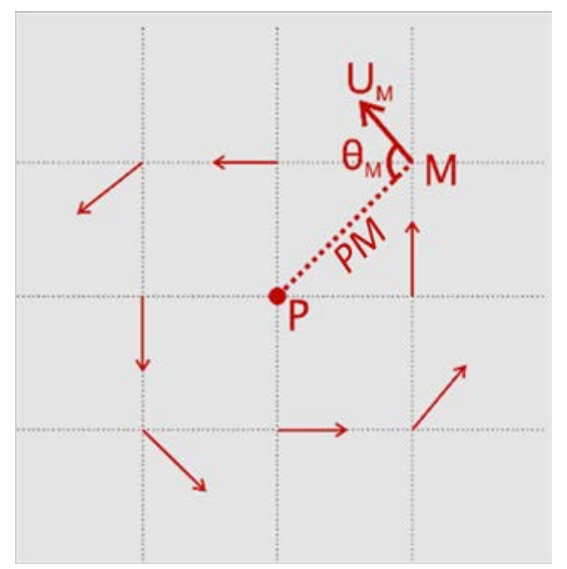

Figure 3.1: Location of variables used in the $\Gamma_{1}$ vortex core identification method [32].

Graftieaux et al [31] state that point $\mathrm{P}$ is considered to be the pinpointed center of an axisymmetrical vortex when the value of $\Gamma_{1}$ at that point $P$ reaches a bound of one. When applied to PIV derived vortex velocity vector fields, the value of $\left|\Gamma_{1}\right|$ generally reaches a local maximum value between 0.9 and 1 . The coordinate location of the point $\mathrm{P}$ associated with the maximum value of $\Gamma_{1}$ is then taken to be the pinpointed location of the core of the vortex. The number of points used in the calculation of $\mathrm{N}$ acts as a spatial filter and helps to remove small-scale turbulent fluctuations [31]. Overall, this approach helps to accurately and consistently pinpoint the center of a vortex.

Graftieaux et al [31] also describe a method that can be used to calculate the boundary of a vortex derived from PIV measured velocity fields. The boundary of a vortex is pinpointed through calculating the dimensionless scalar $\Gamma_{2}$ in Equation 3.2 [31].

$$
\Gamma_{2}(P)=\frac{1}{N} \sum_{S} \frac{\left[P M^{\wedge}\left(U_{M}-\widetilde{U_{P}}\right)\right] \cdot z}{\|P M\| \cdot\left\|U_{M}-\widetilde{U_{P}}\right\|}
$$

$\Gamma_{2}(P)=$ a dimensionless scalar used to calculate the vortex boundary.

$\mathrm{P}=$ the location where $\Gamma_{2}(P)$ is calculated.

$\mathrm{S}=$ the two dimensional area surrounding point $\mathrm{P}$.

$\mathrm{U}_{\mathrm{M}}=$ the velocity vector around the vortex.

$M=$ the point surrounding point $P$ where the velocity vector $U_{M}$ is located.

$\mathrm{PM}=$ radius vector from point $\mathrm{P}$ to point $\mathrm{M}$. 
$\mathrm{z}=$ the unit vector normal to the measurement plane.

$\mathrm{N}=$ the number of points $\mathrm{M}$ inside of $\mathrm{S}$.

$\wedge=$ this symbol represents an exterior product, which is a cross product of PM and $U_{M}$.

$\widetilde{U_{P}}=$ the local convection velocity at point $\mathrm{P}$.

Graftieaux et al [31] explain that the coordinates of point $\mathrm{P}$ represent the boundary of a vortex when the corresponding $\left|\Gamma_{2}\right|$ falls within a range of $0.6-0.7$. If the value of $\left|\Gamma_{2}\right|$ is higher than 0.7 then the coordinates of point $P$ are located within the boundary of the vortex. If the value of value of $\left|\Gamma_{2}\right|$ is less than 0.6 , then coordinates of point $P$ are located outside of the boundary of the vortex. This $0.6-0.7$ range for $\left|\Gamma_{2}\right|$ is chosen since the boundary of a vortex is defined by a $\left|\Gamma_{2}\right|$ value around $\frac{2}{\pi}$. The physics behind this range of $\left|\Gamma_{2}\right|$ values can be seen below [31].

$$
\begin{array}{ll}
\text { if }\left|\Gamma_{2}\right|<\frac{2}{\pi} & \text { the flow is locally dominated by strain (outside the vortex) } \\
\text { if }\left|\Gamma_{2}\right|=\frac{2}{\pi} & \text { the flow is experiencing pure shear (boundary of vortex) } \\
\text { if }\left|\Gamma_{2}\right|>\frac{2}{\pi} & \text { the flow is locally dominated by rotation (inside the vortex) }
\end{array}
$$

The vortex boundary for $\left|\Gamma_{2}\right|$ of about $\frac{2}{\pi}$ can be visualized as an inflection point for $\Gamma_{2}$ in the curve in Figure 3.2. The point 0 on the $\mathrm{x}$ axis of Figure 3.2 represents the exact location of the core of the vortex. The $\frac{r}{l_{0}}$ value along the $\mathrm{x}$ axis measures the radius at which the velocity $U_{\theta}(r)$ has a maximum value relative to the vortex core. The $\mathrm{r}$ is the radius at which the velocity $U_{\theta}(r)$ has a maximum value. The $1_{0}$ is the length of a typical vortex inner core. The $\frac{l}{l_{0}}$ is the length of the area under consideration relative to the typical vortex inner core. Figure 3.2 is generated by Graftieaux et al [31] by analyzing an idealized vortex, a Lamb-Oseen vortex. The sign associated with $\Gamma_{2}$ indicates the direction of rotation for the vortex. Morgan et al [32] compare these results for $\left|\Gamma_{2}\right|$ with 
another Lamb-Oseen vortex with $30 \%$ noise added, Figure 3.3. The goal is to determine if it is possible to accurately identify the bounds of the vortex with the added noise present in the data. The curves in Figure 3.3 with the added 30\% noise have some variability involved but the curves tend to follow the correct trend and the maximum values of each curve approach a $\left|\Gamma_{2}\right|$ value of one. Overall the noise does not seem to affect the $\frac{l}{l_{0}}$, or $\frac{r}{r_{0}}$, curves since it is a global scheme. The red dashed curve in Figure 3.3 seems to be affected the most by the added noise. This curve has a $\frac{r}{r_{0}}$ value of 0.125 , which is the lowest value considered in this analysis. This means that as the interrogation grid becomes smaller and smaller, the global approach of the $\left|\Gamma_{2}\right|$ vortex boundary identification method begins to act more like a local scheme. Therefore, if the interrogation grid becomes too small, the $\frac{r}{r_{0}}$ curves will be greatly affected by the noise [32].

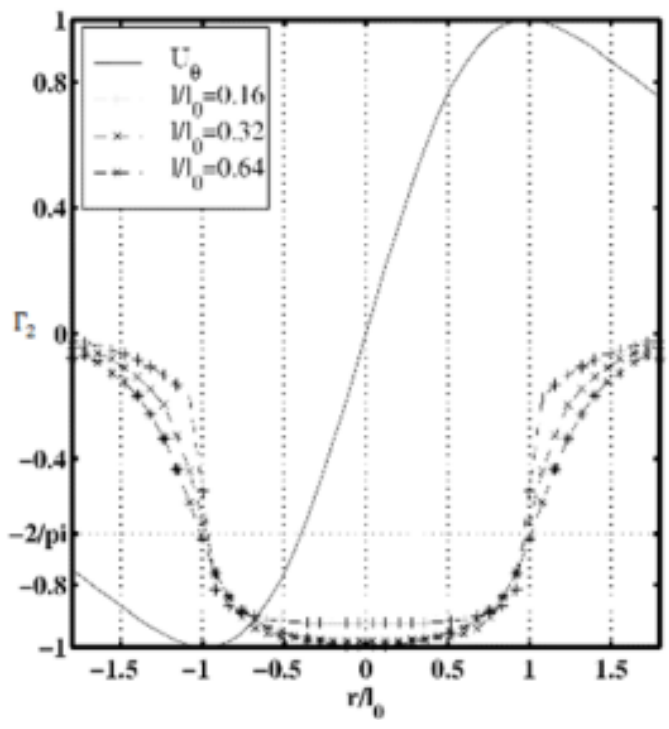

Figure 3.2: Results of the $\Gamma_{2}$ method used to identify the boundary of a vortex [31].

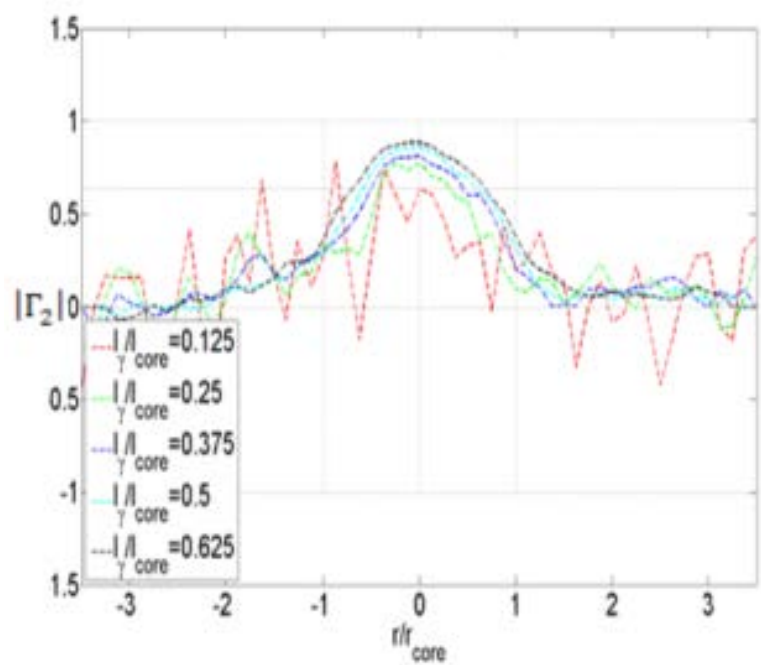

Figure 3.3: Results of the $\Gamma_{2}$ method used to identify the boundary of a vortex with $30 \%$ noise added [32]. 
In summary, the $\Gamma_{1}$ and $\Gamma_{2}$ vortex identification method results can clearly be seen in a paper by Baik et al [33]. In this research, $\Gamma_{1}$ and $\Gamma_{2}$ are used to identify the center of the vortex as well as the vortex bounds for the wake of a pitching and plunging flat plate at low Reynolds numbers. Figure 3.4 shows the same velocity vector fields with the contours representing the vortex center for $\Gamma_{1}$ and the boundary of the vortex for $\Gamma_{2}$.

Several secondary vortices also exist behind the primary vortex in this figure [33].
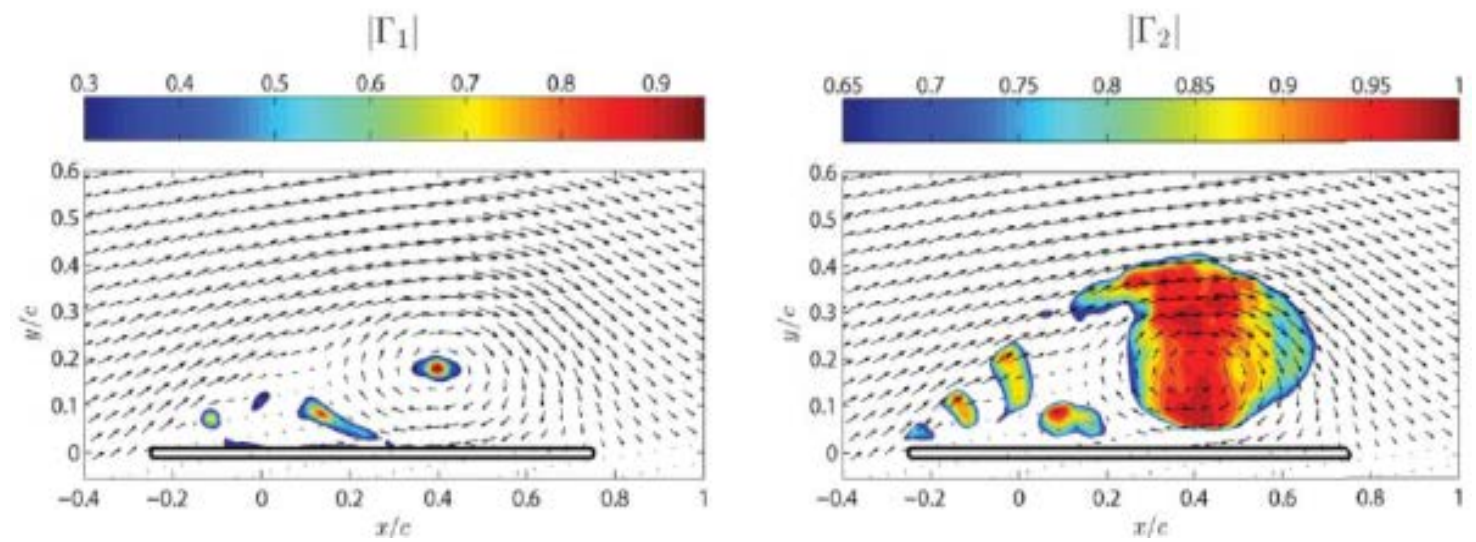

Figure 3.4: Contours for vortex centers from $\Gamma_{1}$ and the vortex boundaries from $\Gamma_{2}[33]$.

Overall the results show that the vortex identification functions using $\Gamma_{1}$ and $\Gamma_{2}$ can accurately locate the vortex center and boundary in a velocity field. It is also demonstrated that these functions can also accurately identify a vortex in the presence of small-scale turbulence that is sporadically embedded within the vortices. This same theory for identifying the vortex center and boundary will be used for the MAV wingtip vortex research in this thesis. Since the vortices in this thesis research are derived from experimental PIV testing, noise will be present in the data used to calculate vorticity. The ability to accurately identify the vortex core and boundary amongst this noise is 
imperative in obtaining consistent results for calculations performed on these vortices, such as the circulation.

\subsection{Methods of Calculating the Circulation of a Vortex}

Several different methods can be used to calculate the circulation of a vortex from a velocity field. Hong and Altman [11] calculate the circulation of a vortex, $\Gamma_{z}$, by taking the integral of the z-vorticity, $\omega_{z}$, over the area of a vortex, dS. See Equation 3.3.

$$
\Gamma_{\mathrm{z}}=\int \omega_{\mathrm{z}} \cdot \mathrm{dS}
$$

In their book, Katz and Plotkin [34] describe how the circulation value for a vortex can also be found by using the line integral of the velocity, $\mathrm{V}$, over the closed curve, dl, of the vortex, see Equation 3.4 [34].

$$
\Gamma_{\mathrm{z}}=\oint_{C} \vec{V} \cdot d l
$$

Baik et al [33] produce a plot in Figure 3.5 that compares the results for the two circulation calculation methods applied to PIV experiments. The calculated values are essentially the same. In this thesis research, both of these methods will be used to calculate and compare the circulation of the MAV wingtip vortices. 


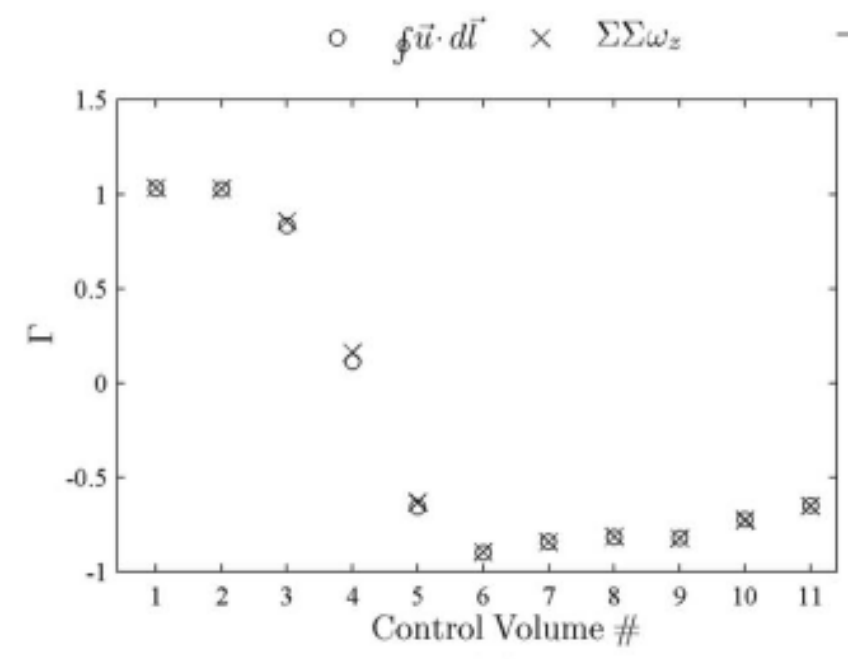

(a)

Figure 3.5: Comparison of results for two methods used to calculate the circulation for a vortex [33].

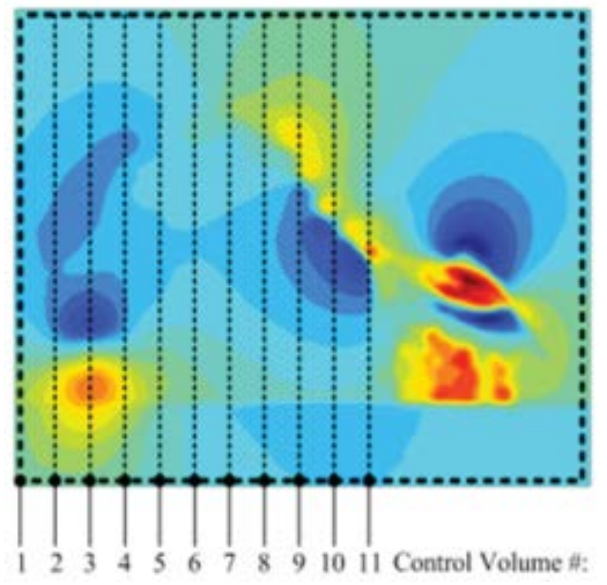

Figure 3.6: Variation of control volume for circulation calculations [33].

Figure 3.5 is a valuable plot, because it also demonstrates that the circulation value of a vortex changes depending on the size of the control volume that is used in the calculation. Figure 3.6 shows the sizes of the control volumes overlaid on the vortex image [33]. The control volume labeled " 1 " on the $\mathrm{x}$ axis in this plot is the largest control volume encompassing the entire vortex field, whereas the control volume labeled " 11 " is the smallest control volume because it only uses the volume to the right of that line. Together, these images show that for this test, the circulation values rise for the larger control volume. The best results are found to lie in control volumes five, eight, and nine since the corresponding lift and drag coefficients are similar and the errors associated with those control volumes are small compared to the other volumes. This circulation sensitivity analysis relates to the wall effects research in this thesis since a similar sensitivity analysis based on the size of the control volume used for the circulation calculation will be completed although the imaged flow field is considerably simpler than that seen in the figure above. 


\subsection{Methods of Calculating the Lift Force from a Vortex}

Lift is created by the wingtip vortex $[13,35]$. The goal of this wall effects research is to quantify how the lift of the airplane is affected by the wingtip vortex interaction with a wall.

Hong and Altman [11] discuss the vortex lift contributions in the spanwise plane of a wing by looking at the wingtip vortices of flapping wings. They show that once the circulation of a vortex is known through using equations like Equation 3.3 or 3.4, the lift can be estimated from the Kutta-Joukowski theorem. See Equation 3.5. The span term, $\mathrm{b}$, is the effective span and is measured as twice the distance between the visible core of the wingtip vortex and the center plane of the wing. $\mathrm{L}$ is the lift and the $\mathrm{V}$ is the flight velocity. Finally, $\Gamma_{\mathrm{z}}$ is the circulation [11]. This approach will be used in the MAV wingtip wall effects research to calculate the lift force in the wingtip vortex after the circulation has already been calculated. This approach is chosen since it serves as the simplest and most direct calculation of lift from a vortex.

$$
L=\rho V \Gamma_{\mathrm{z}} \mathrm{b}
$$

While the Kutta-Joukowski theorem demonstrates one method to calculate the lift from a vortex, several other methods do exist. In a paper by Noca et al [36] the force exerted by a fluid flow on a body is investigated. Three equations are developed and are referred to as the "impulse equation", the "momentum equation", and the "flux equation." These equations are advantageous because they rely on information from the velocity field and its derivatives in an arbitrary, finite control volume. Overall, the three approaches described in this paper yield accurate force calculations for flows with large 
force coefficients $(\sim 5)$, but future work would be necessary to validate the approaches for flows with smaller force coefficients $(\sim 0.2)$ [36].

In a different paper, Hong and Altman [37] discuss several methods that can be used to calculate the aerodynamic forces on flapping wings by using velocity or vorticity fields. The equation they use is uniquely developed by Lin and Rockwell in PIV experiments [38] and is cited from Lighthill [39]. Lighthill determines the instantaneous lift per unit length for a two-dimensional flow by using the excess, or shed, vorticity. Lin and Rockwell [38] compare Lighthill's approach to the extended Blasius theorem to determine contributions to instantaneous lift from instantaneous vorticity fields from vortices. Both methods result in successful techniques to identify the areas of a vortex where the vorticity contributes to the instantaneous lift in the near wake of an object. While Hong and Altman [37] use these methods for the wake of flapping wings, it could also be used for the wake from a fixed wing aircraft.

All of this research into the lift force that the vortex produces is relevant to the wall effects research in this thesis because it will be necessary to determine how the lift from the vortex near a wall affects the MAV and its flight controls as it flies along the wall. Some limitations with this approach involve the fact that the three-dimensional phenomenon of a wingtip vortex tube is being considered in a two-dimensional snapshot frame. The two-dimensional vortex that is considered in each test is also located a certain distance downstream from the aircraft that it is imparting a force against. 


\subsection{Wingtip Vortex Ellipticity}

The elliptical instability between two vortices that Le Dizes and Laporte [24] discuss can be applied to the wall effects research in this thesis. During the free flight tests along the wall, the wingtip vortex is constricted due to the presence of the wall. The constriction of the vortex causes the wingtip vortex to take on an elliptical shape due to the three-dimensional instability properties described by these authors. For more detail refer back to Chapter two of this thesis. The wall in this thesis testing can serve as the mirror vortex to the wingtip vortex. The elliptical shape of the wingtip vortex near the wall helps to identify the effect of the wall on the wingtip vortex.

Potylitsin and Peltier [25] describe the location of three-dimensional elliptical instabilities within Stuart vortices. This research applies to the wall effects of wingtip vortices research in this thesis because identifying where three-dimensional elliptical instabilities exist for the wall effect wingtip vortices is crucial. This helps to understand and compare the wingtip vortex circulation of the wingtip vortices with and without a wall in close proximity. As described in the background research, the Stuart vortex with moderate ellipticity has a dominant instability located at the core of the vortex as well as a weaker additional instability on the edge of the vortex boundary. In contrast, the Stuart vortex with low ellipticity has a dominant instability located at the boundary edge of the vortex and an additional weaker instability at the vortex core. Applying this same theory to the wingtip vortices in this thesis research leads to the thought that the wingtip vortices near the wall could be considered to have a moderate ellipticity and the wingtip vortices away from the wall could have low ellipticity. These levels are assigned to the experimental tests in this thesis by comparing the degree of ellipticity of the wingtip 
vortex vorticity contour plots to Figure 2.12. Since the wingtip vortex near the wall is considered to have moderate ellipticity, this type of vortex should have a dominant threedimensional instability located at the core as well as an additional weaker threedimensional instability located on the edge of the vortex boundary. This makes sense since the core of the wall vortex is very elliptical, there must be a strong instability located at the core in order to deform the vortex core this drastically. In contrast, since the wingtip vortex without the wall present is considered to have low ellipticity, this type of vortex should have a dominant three-dimensional instability located at the boundary edge of the vortex and an additional weaker instability located at the vortex core. This is reasonable since the wingtip vortices without the wall present should have a circular shape and the stronger instability is located at the vortex boundary and does not deform the shape of the vortex.

Once the general locations of these three-dimensional instabilities are known for the wingtip vortices with and without a wall present, their effects can be analyzed by looking at the circulation values for these tests. While these instabilities are threedimensional in nature, the two-dimensional PIV images can still capture two of the three components of the instability. The wall area and line integration circulation values should not be as consistent as the no wall circulations values because the dominant instability for these wall vortices of moderate ellipticity will occur at the vortex core. This instability is always included in the area integration centered on the vortex core and the line integral passing around the edge of the vortex core. While the very center core of the vortex does not always contain a significant number of velocity vectors in the PIV images, the $\Gamma_{1}$ criteria helps to define the bounds of the core of the vortex and these 
bounds exceed the areas of the core where only a few velocity vectors exist. Therefore, the vortex core still has enough velocity vectors to contain the properties of the instabilities present in the vortex core. The additional weaker edge instability is not included in the area or line integration because this instability is considered to be located outside of the $\Gamma_{2}$ vortex boundary used to define the area and radius of the vortex for the area and line integration circulation calculations.

In contrast, the area and line integration circulation values for the tests conducted without a wall present should be consistent because the max instability for these circular vortices of low ellipticity occurs in the edge instability ring surrounding the edge of the vortex. This instability is not included in the area integral or line integral since this instability is located in vortex edge outside the $\Gamma_{2}$ vortex boundary used to define the area and radius for the area and line integration circulation calculations.

Based on the theory discussed above, it is possible to see the effect of the wall on the shape and stability of the wingtip vortex by comparing the consistency of the wingtip vortex circulation values for the tests completed with or without a wall present.

\subsection{Particle Image Velocimetry (PIV)}

Particle image velocimetry is a nonintrusive testing method that can be used to analyze flow fields. Often times it is used to visualize and perform calculations for the flow over an object placed in a wind tunnel. Instantaneous velocity vector measurements can be made for the flow and two velocity components are calculated for twodimensional PIV and three velocity components are calculated for three-dimensional PIV, or stereo PIV. The basic idea behind PIV involves seeding the air with particles, 
using a pulsing laser sheet to illuminate the particle, and then capturing the movement of those particles with a high speed camera. This camera captures each laser pulse in a separate image frame and the particles' movement is tracked between the two images. These images are subdivided into small sections called interrogation regions. These regions are then cross correlated with each other over every pixel and the velocity vector field for the two images is created. Figure 3.7 shows a detailed visual explanation of PIV [40].

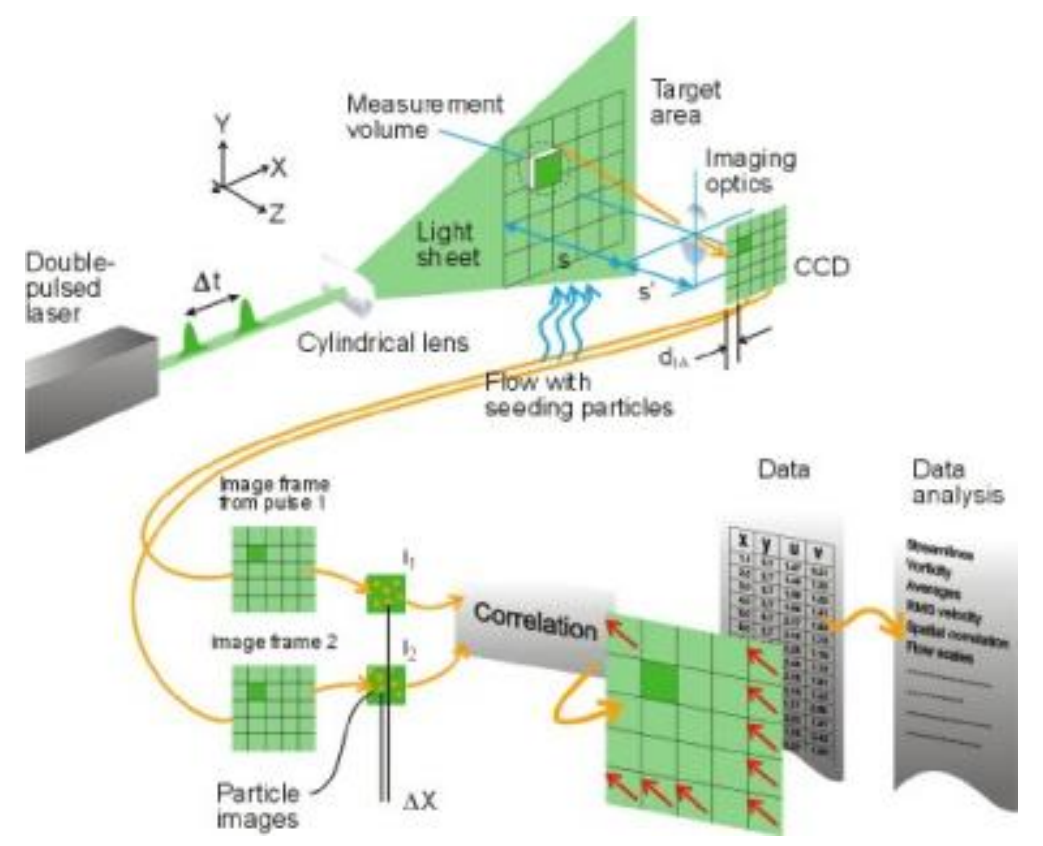

Figure 3.7: Flow chart showing the theory behind a PIV test setup [40].

\subsubsection{PIV Seeding}

The seed for PIV in air is often made of oil or water based smoke particles from a smoke machine. These particles are injected into the air and the laser sheet illuminates the seed so that the high speed camera can focus on individual smoke particles. Correct PIV seeding is imperative for obtaining accurate PIV results. If the smoke particles are 
not uniformly mixed or the area is saturated with particles, structures of seed can form and the high speed camera will not be able to distinguish between individual smoke particles and groups of particles. A lack of seeding within the high speed camera's field of view can also lead to errors in the PIV velocity vector field. There are a variety of materials and particles sizes that can be used for the seed material. See Table 3.2. The appropriate camera lens and distance from the camera to the laser sheet should be set so that the smoke particles are about two pixels in diameter when viewed in the raw PIV images. This is a desirable size for the particles because it will lead to the lowest RMSuncertainty for cross correlation PIV algorithms as shown in Figure 3.8 [41].

Table 3.2: Seeding particles in gas flows [41].

\begin{tabular}{llll} 
Material & $\begin{array}{l}d_{\rho} \\
(\mu \mathrm{m})\end{array}$ & Laser & $\begin{array}{l}\text { Pulse energy, } \\
\text { pulse time }\end{array}$ \\
\hline $\begin{array}{l}\mathrm{TiO}_{2}(m=2.6, \\
\left.\rho=3500 \mathrm{~kg} \mathrm{~m}^{-3}\right)\end{array}$ & $<1$ & Nd:YAG & $10 \mathrm{~mJ}, 20 \mathrm{~ns}$ \\
$\mathrm{TiO}_{2}, \mathrm{ZrO}_{2}$ & $0.7-1$ & $\mathrm{Nd}: Y A G$ & $110 \mathrm{~mJ}, 12 \mathrm{~ns}$ \\
$\mathrm{Al}_{2} \mathrm{O}_{3}(m=1.76$, & 0.3 & $\mathrm{Nd}: Y A G$ & $400 \mathrm{~mJ}$ \\
$\left.\rho=3970 \mathrm{~kg} \mathrm{~m}^{-3}\right)$ & 3 & $\mathrm{Nd}: Y A G$ & $9 \mathrm{~mJ}, 6 \mathrm{~ns}$ \\
& 0.8 & Ruby & $20 \mathrm{~ns}$ \\
Polycrystalline & 30 & Nd:YAG & $135 \mathrm{~mJ}, 6 \mathrm{~ns}$ \\
Glass & 30 & Ruby & $30 \mathrm{~mJ}, 30 \mathrm{~ns}$ \\
Oil smoke & 1 & Ruby & $5 \mathrm{~J}$ \\
Corn oil & $1-2$ & Nd:YAG & $100 \mathrm{~mJ}$ \\
Oil & $1-2$ & Nd:YAG & $120 \mathrm{~mJ}$ \\
Olive oil & 1.06 & Nd:YAG & $70 \mathrm{~mJ}, 16 \mathrm{~ns}$ \\
$\quad(m=1.47$, & & & \\
$\left.\rho=970 \mathrm{~kg} \mathrm{~m}^{-3}\right)$ & & &
\end{tabular}




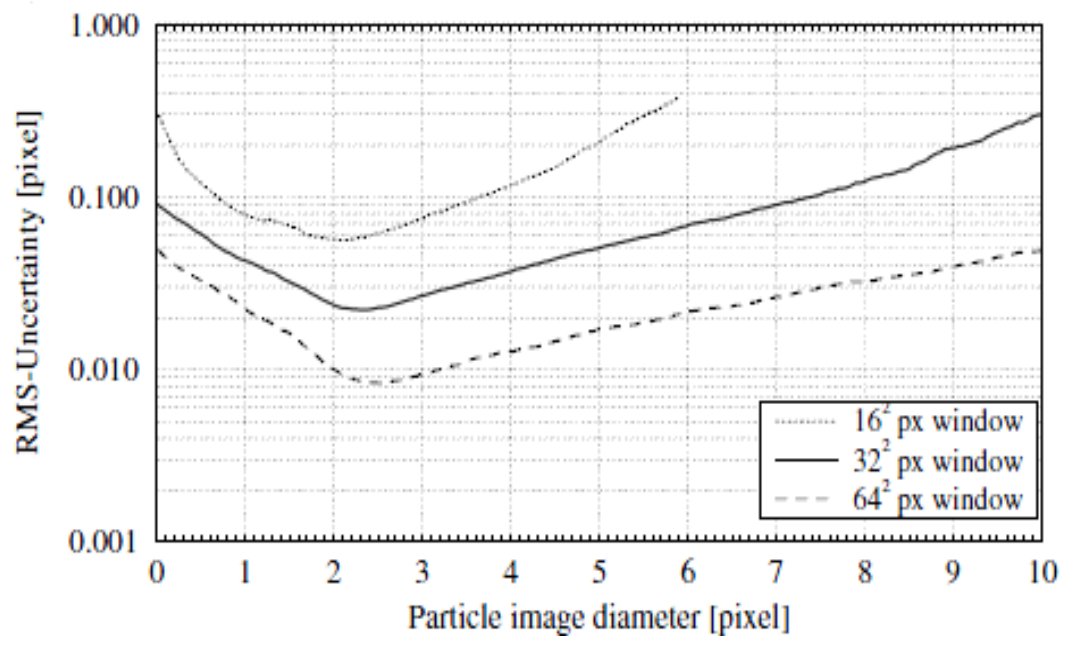

Figure 3.8: RMS-uncertainty vs. the particle image diameter for cross correlation PIV [41].

\subsubsection{Laser and Optics}

A double pulsed laser helps to illuminate the smoke particles in PIV testing. The delta $t$ value that is seen in Figure 3.7 has to do with the frequency of the laser pulse. Every time that the laser pulses, the camera is able to record one pair of images. The pulse rate can be adjusted according to the type of testing that is performed. Some of the different laser types and pulse energies used in PIV can also be seen in Table 3.2. A $\mathrm{Nd}$ :YAG (neodymium-doped yttrium aluminium garnet) laser is a very common light source used for PIV. A ruby laser could also be used and this is a solid state laser that uses a synthetic ruby as the laser medium [42]. For PIV applications, the laser beam is generally focused into a thin laser sheet through the use of optical lenses.

Most PIV optical configurations consist of a spherical lens and a cylindrical lens (plano-concave lens). These lenses help to focus and spread the laser beam with minimal power intensity losses. These optics help to create a thin laser sheet that will only illuminate the particles within the focus plane of the high speed camera [43]. 


\subsubsection{PIV Cameras}

A popular high speed camera used for PIV testing is a charge coupled device (CCD) high speed camera. A charge coupled device sensor converts light into an electric charge. The resolution of these cameras can be above 4,000 by 4,000 pixels. A dual shutter capability in these cameras gives them the ability to capture images of the seed particles in two separate frames, which will be combined in the cross correlation algorithms. See the two frames in Figure 3.7. Another delta $t$ time value is the time delay between these two frames. These cameras can have delta $t$ values shorter than one microsecond. This delta t time value is important because it is the time value that is used in combination with the seed displacement to calculate the velocity vectors. If this time delay value is too short, the camera will not see the seed particles change position over that time period. If the delta $t$ value is too long, the seed particles may leave the processing interrogation region before the second image is recorded. A general rule of thumb when determining the correct delta $t$ value for a PIV test, involves using a value that gives a pixel displacement between the two frames that is about $1 / 4$ to $1 / 3$ of the distance of the smallest interrogation region used. For example, a 64 by 64 interrogation region could be used first when processing the PIV data and a 32 by 32 interrogation region could follow. Therefore, the ideal seed particle pixel displacement between the two frames would be about eight to ten pixels since this is about $1 / 4$ to $1 / 3$ of the $32 \times 32$ pixel interrogation region. A good starting point for the delta $t$ value for a PIV experiment can be calculated through following the steps listed below.

1. Divide the length of the camera's field of view distance by the number of pixels in the primary flow direction. 
2. Multiply that distance per pixel by the pixel displacement desired for the test based on the smallest interrogation region.

3. Divide this distance by the velocity of the flow under consideration and the resultant time value is the ideal delta $t$ value.

While this calculation will help to define a good starting point for the delta $t$ value, this time value may need to be tuned during testing to obtain the ideal pixel displacement due to the presence of velocity gradients across the field of view in most flow fields of interest [41].

Overall, PIV has proven to be an excellent quantitative testing method to analyze aerodynamics. PIV will be used in this thesis to look at the aerodynamics of a MAV's wingtip vortex in free flight as it flies in close proximity to a wall.

\subsection{Micro Air Vehicle Indoor Flight Facility}

The free flight PIV experiments for this thesis are conducted at the Air Force Research Labs Micro Air Vehicle Integration and Application Research Institute ( $\mu$ AVIARI) indoor flight test lab at Wright-Patterson AFB in Dayton, Ohio. The 55' X 70' open flight room can be seen in Figure 3.9 and contains a total of 60 Vicon motion capture cameras mounted at two elevations around the entire perimeter of the room. This facility is currently the world's largest operational facility of its kind. The motion capture cameras emit light through red LEDs and strobe at a predefined frequency of 125 Hz. for this test. An example of an individual Vicon camera can be seen in Figure 3.9. The light from these cameras bounce off reflective markers placed on the object that requires tracking and are taken back into the camera through the camera sensor. The cameras triangulate sub millimeter position data for where the marker is located in the 
room in all three dimensions. This position data can be used to calculate velocity and acceleration measurements. See Figure 3.9 for an example image of the output from the Vicon software that is used to locate each individual camera and show its orientation within the flight room.

The control room can be seen in Figure 3.10 and consists of two state of the art computer workstations to monitor the activities in the flight room.

For this research, the aircraft chosen to perform flight testing in close proximity to a wall is the commercially available ParkZone Night Vapor RC aircraft, seen in Figure 3.11 with four reflective markers. See Figure 3.12 for an image demonstrating how the Night Vapor appears in the Vicon camera software. See Table 3.3 for the Night Vapor's specifications. This aircraft is chosen for this research due to its inherent stability characteristics at low enough speeds to fly comfortably indoors and its availability from local hobby shops.

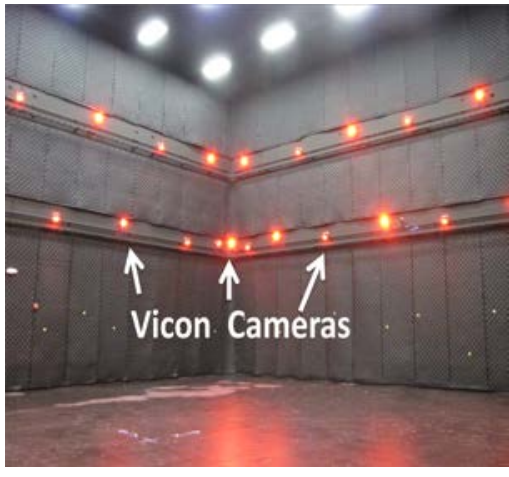

Figure 3.9: Flight room, Vicon camera example, and Vicon software to show virtual location of each camera.

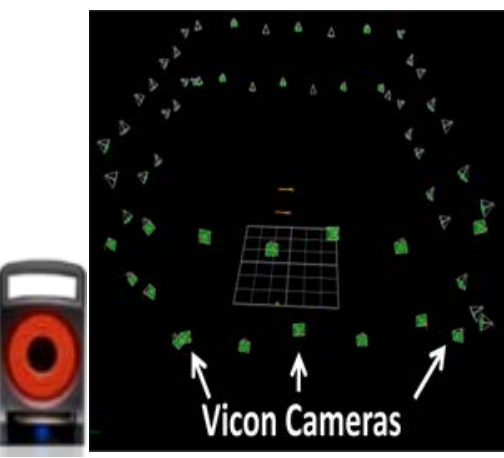

Figure 3.10: Control room. 


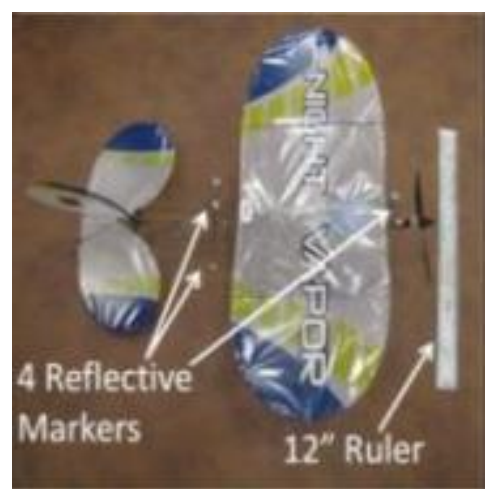

Figure 3.11: Night Vapor.

12 " ruler for scale.

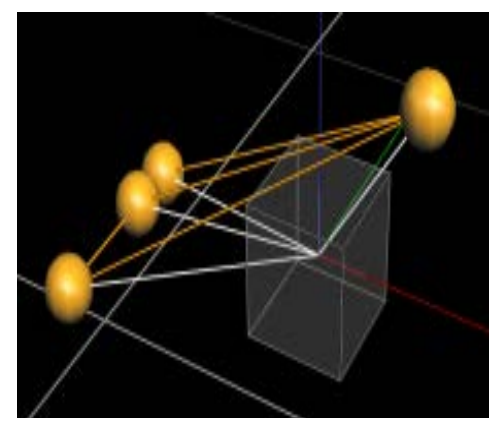

Figure 3.12: Reflective markers on aircraft as seen in Vicon software.

Table 3.3: Details for the ParkZone Night Vapor RC aircraft.

\begin{tabular}{|c|c|}
\hline \multicolumn{2}{|c|}{ ParkZone Night Vapor Specifications } \\
\hline Wingspan (cm.) & 36.83 \\
\hline Overall Length (cm.) & 39.37 \\
\hline Flying Weight (g.) & 21 \\
\hline Prop Size (mm. X mm.) & $140 \times 45$ \\
\hline Battery Type & $70 \mathrm{mAh}, 3.7 \mathrm{~V}, 14 \mathrm{C} \mathrm{Li-Po}$ \\
\hline Reynolds Number & $16,000-30,000$ \\
\hline Aspect Ratio & 2.74 \\
\hline
\end{tabular}

\subsection{Wind Tunnel Testing}

The reasoning behind wind tunnel testing in this thesis research is to measure the lift and drag on the aircraft to determine if that lift is similar to the lift estimated from the wingtip vortex wake from the free flight PIV tests. In order to do this, the Night Vapor is mounted on a sting balance in the University of Dayton's low speed wind tunnel. A plexiglass wall structure was mounted into the wind tunnel to simulate the wall that the Night Vapor flew along. The same tests that were listed for the free flight tests in Table 3.1 were repeated in the wind tunnel at fast and slow flight velocities. For the wall tests, the wall was placed as far away as possible and then incrementally translated closer and 
closer to the Night Vapor's wingtip in order to measure the wall effect on the lift force of the aircraft at various wall positions. This provides insight into how close to the wall the wingtip must be in order to observe a measurable change in the calculated coefficient of lift. Overall, the wind tunnel testing serves as a form of validation for the free flight PIV tests by increasing the number of controlled variables in the experiment. 


\section{CHAPTER 4}

\section{EXPERIMENTAL SETUP}

\subsection{PIV Proof of Concept Experimental Setup}

A full scale proof of concept test is successfully completed to determine if the wingtip vortices of the Night Vapor can be tracked through PIV testing and to see how well the Vicon cameras can track the Night Vapor during the PIV test. Figure 4.1 shows the proof of concept test setup in the $\mu$ AVIARI flight room. An ad-hoc cardboard wall is constructed and hung from a truss. The wall is placed in the center of the room where better Vicon camera coverage is present and the Night Vapor can be tracked even though the wall blocks some of the cameras. The position data from the Vicon cameras is collected through using a server and a LabView interface located in the control room. The PIV laser is a Quantel Big Sky laser Twins BSL 200 model NdYAG Q-switched multipulse green laser and can be seen in Figure 4.2. A Le Maitre G300 smoke machine can also be seen in Figure 4.2 and is used in haze mode to produce smoke particles with a 10 micron scattering diameter. The LaVision optical assembly consists of a spherical lens, a plano-convex lens, and a -6 mm plano-concave lens. This laser sheet originates from the laser beam which is positioned parallel to the ground. The beam is reflected 
vertically off a $45^{\circ}$ mirror and then enters the optics to create the laser sheet. The smoke seems to drift in and out of the laser sheet, so a cardboard channel is constructed to direct the flow of smoke into the laser sheet. A $55 \mathrm{~mm}$ Nikon lens is used on a PCO 1600 double-shutter CCD camera with a $1600 \times 1200$ pixel array. This camera is positioned perpendicularly to the laser sheet and captures the flow of the smoke particles by taking pictures every $0.1 \mathrm{~s}$ according to the laser repeat frequency of $10 \mathrm{~Hz}$. A delta $t$ value of $3.5 \mathrm{~ms}$ is used. The camera is positioned $2.74,2.29$, and $1.83 \mathrm{~m}(9,7.5$, and $6 \mathrm{ft})$ away from the laser sheet. The $2.29 \mathrm{~m}$ camera position provided the best field of view for capturing the vortex as it propagates and interacts with the wall. The laser sheet is also focused for the first tests but then purposely defocused in order to allow a greater illumination of particles moving in the out of plane direction which is required by the magnitude of the axial flow [out of plane] through the core of the vortex. A photo detector with a laser pointer is used in an attempt to automate the PIV test by triggering the laser and PCO 1600 camera when the Night Vapor breaks the laser beam. However, the Night Vapor's fuselage does not have a significant surface area to reliably break the laser beam and trigger the PIV event. The photo detector is also not sensitive enough to work with the laser pointer's weak strength and thus this automation attempt did not work. 


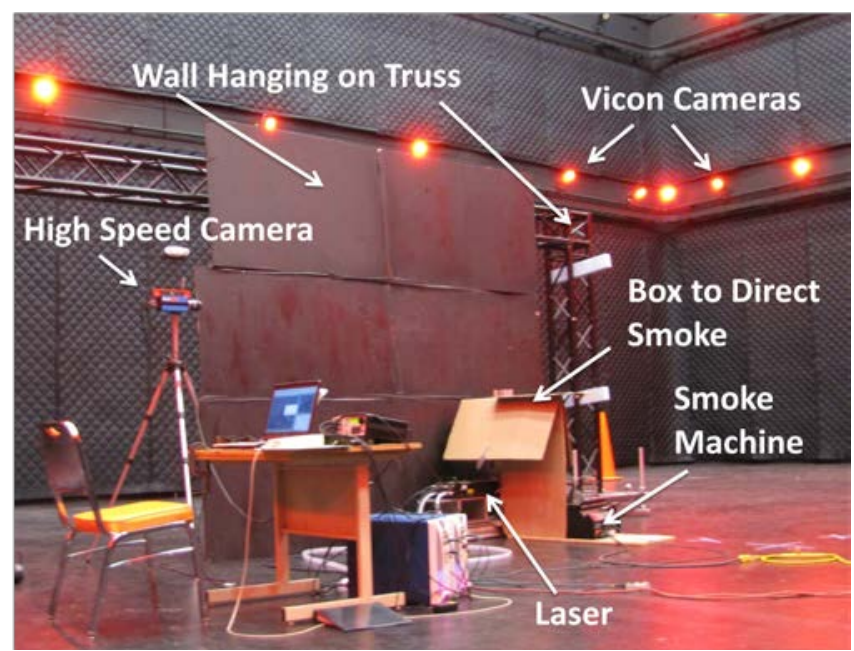

Figure 4.1: Test setup in the middle of the room. The brown cardboard box around the laser, optics, and smoke machine, directed the smoke into the laser sheet.

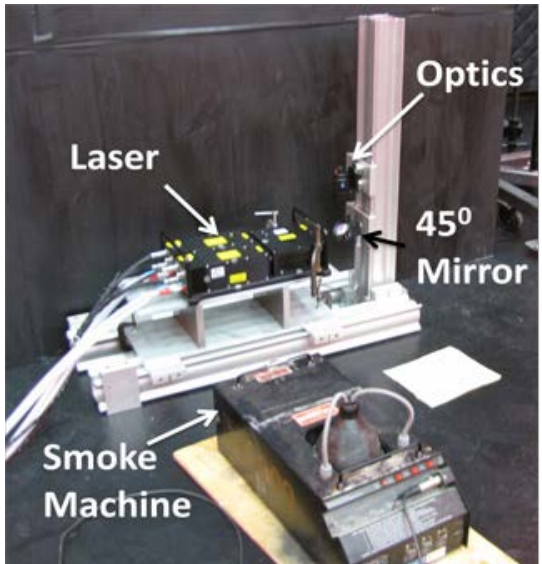

Figure 4.2: The laser, $45^{0}$ mirror, optics, and smoke machine are shown here with the box removed.

\subsection{Improving PIV Seeding}

As will be discussed in further detail in the results section of this thesis, the proof of concept experimental setup shows that the Night Vapor's wingtip vortex can be visualized through PIV testing and that the Vicon motion capture cameras can accurately track the velocity of the Night Vapor during PIV testing. However, the PIV seeding method needs significant improvement to be able to provide accurate PIV measurements of the wingtip vortex which can be used to calculate the circulation and lift force of the vortex. About three weeks of testing time is dedicated to improving the method of creating a uniformly dispersed smoke field for the PIV test. The problem lies in the seeding of a seemingly quiescent flow in the $55^{\prime} \mathrm{X} 70^{\prime}$ open flight room. The resulting natural convective currents within the room tend to transport the seed away from where it is needed. Cardboard boxes, baffles, fans, chimneys, alternative smoke machines, and dry ice are also used in different combinations in an attempt to contain the smoke and 
allow it to mix, cool, and homogeneously disperse before entering the laser sheet. All of these attempts did not provide the repeatable uniformly dispersed seeding that is ideal for PIV. It is also not desirable to fill the entire 55' x $70^{\prime}$ flight room full of smoke particles because the operator of the test would have very little control over the seeding. In addition the manufacturer of the Vicon cameras could not provide information regarding the possibility of damage to the motion capture camera sensors as a result of interacting with the smoke particles over long periods of time. The final test setup, which provides the best PIV smoke particle mixing, is discussed next.

\subsection{Final PIV Experimental Setup}

Figures 4.3-4.7 show the final test setup in the $\mu$ AVIARI flight lab. Figure 4.3 shows the top view of the final test setup, which involved creating an enclosed room to contain the smoke and allow it to mix uniformly. Orange arrows in Figure 4.3 show the flight pattern of the Night Vapor through the room structure. The Night Vapor manually flies into the room through an opening that is $0.91 \mathrm{~m}$ tall by $1.04 \mathrm{~m}$ wide. Once inside the room, the Night Vapor flies along the wall through the laser sheet and the PIV camera's field of view, and then out of the room through the same size opening it flew through to get into the room. An ad-hoc cardboard wall is used and is hung from a truss. The cardboard wall is advantageous because it allows the freedom to cut openings in the wall for the many different seeding techniques tested while trying to create a more uniform seeding. A plexiglass wall is located close to the wall and forms the bulk of the room's structure. Additional plexiglass sheets are suspended to form the roof of the room. Originally there was some uncertainty as to whether the Vicon cameras would be 
able to accurately track the vehicle while flying through the plexiglass enclosure. After performing multiple test flights through the structure, it is determined that the plexiglass room did not significantly alter the recorded results. If the Vicon cameras did lose the Night Vapor during a test, the data would begin recording again when the aircraft is recognized again. Several Vicon cameras are also able to look into the room through the windows that the Night Vapor flies though. Through looking at the data, it is determined that for the tests where the Night Vapor is lost, it is only lost for about $0.3 \mathrm{~s}$ and any error induced from a dropped Vicon frame could be averaged out with a 20 point moving average on the velocity measurements, assuming no violent maneuvers are undertaken by the Night Vapor.

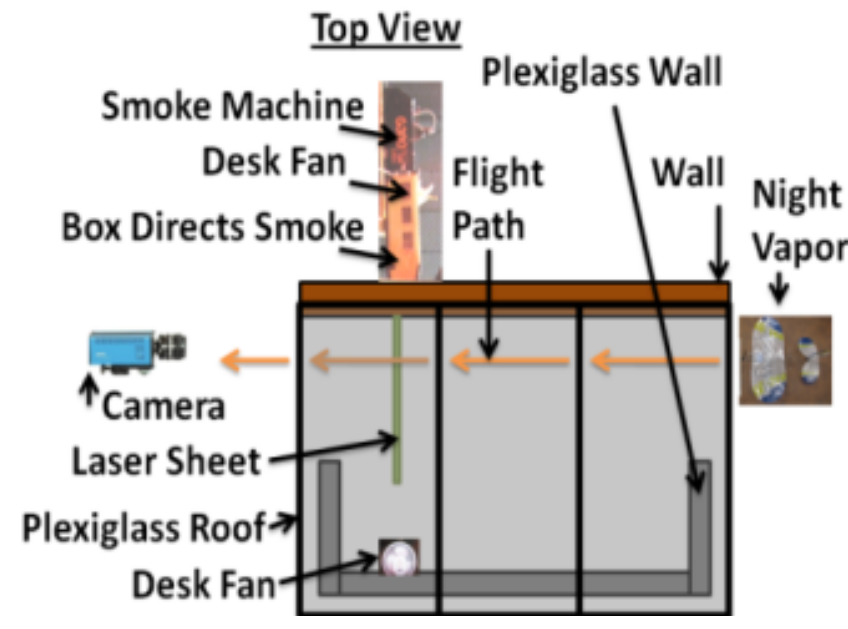

Figure 4.3: Top view of small room to fly through.
Left Side View Night Vapor Flight Out of Page Box to Direct Smoke / Plexiglass

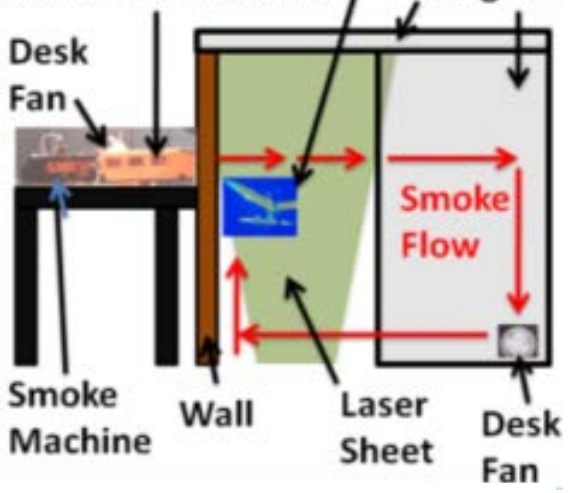

Figure 4.4: Side view of wall test setup in flight room. 

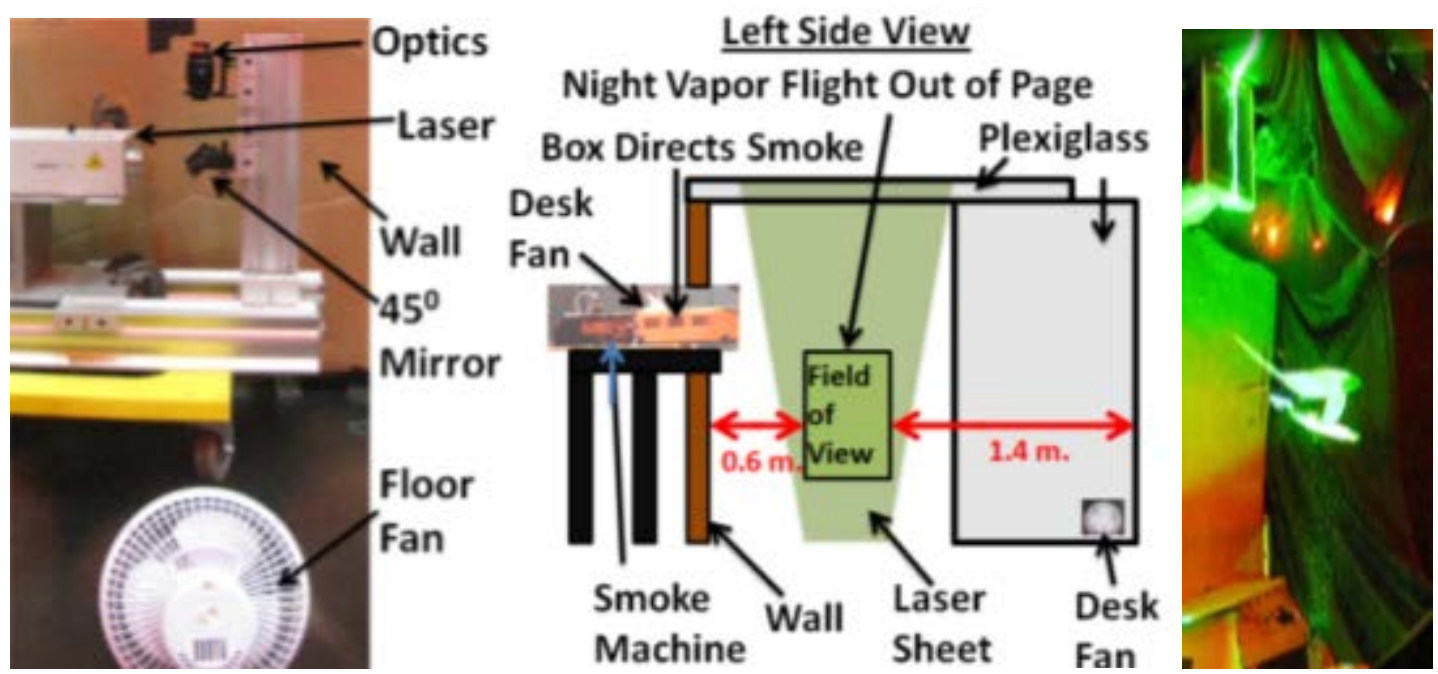

Figure 4.5: Laser and optics.

Figure 4.6: Test setup to contain smoke but allow the aircraft to fly through the room without the wall having a significant effect on aircraft wingtip vortex.

Figure 4.7:

Flying through laser: far away from wall.

Figure 4.3 also shows the location on the wall where the smoke enters the room.

The smoke machine is located behind the wall and can be seen in the left side view of the test setup in Figure 4.4. This figure also shows the position of a six inch diameter desk fan and a cardboard channel that is used to direct the smoke into the room. When the smoke passes through an opening in the wall, it passes through three flow normalization screens which also help to create a uniform smoke field. Figures 4.3 and 4.5 also show a second small six inch diameter desk fan located on the floor of the plexiglass room. The red arrows in Figure 4.4 show the flow pattern of the smoke particles. The first desk fan pushes the smoke through the screens and into the room. The second desk fan on the floor pulls the smoke particles down to the floor and then pushes them into the laser sheet and up the wall creating fine uniformly distributed PIV seeding along the wall. It should be noted that once uniform seeding is obtained the fans and smoke machine are turned off and the bulk flow allowed to damp out. This damping is verified for each case with the 
acquisition and evaluation of numerous image pairs before the Night Vapor flies through the cross-plane field of view.

Figure 4.5 shows the laser, the $45^{\circ}$ mirror, and the LaVision optics that are used to create the laser sheet. The optical assembly is the same assembly described in the proof of concept testing in section 4.1. The laser used for this testing is a Nd:YAG Dual Cavity pulsed laser Litron nano L 200-15 model with $200 \mathrm{~mJ} /$ pulse at $532 \mathrm{~nm}$. The left side view in Figure 4.6 shows the test setup for the tests completed far away from the wall. The wall is positioned $0.6 \mathrm{~m}$ away from the left most edge of the camera's field of view. The plexiglass wall remains in its original location and is $1.4 \mathrm{~m}$ away from the camera's right most field of view. These distances are deemed to be sufficiently large to not have a significant effect on the flow of the wingtip vortices since they are more than five wingtip vortex diameters away from the edge of the camera's field of view. The largest value of a wingtip vortex core diameter is found to be $0.075 \mathrm{~m}$ and five times this diameter gives a distance of $0.375 \mathrm{~m}$.

Figure 4.3 also shows the $55 \mathrm{~mm}$ Nikon lens that is used on the PCO $1600 \mathrm{CCD}$ double-shutter camera. The camera is positioned perpendicularly to the laser sheet and captures the flow of the smoke particles by taking pictures every $0.083 \mathrm{~s}$ with the laser pulse frequency of $12 \mathrm{~Hz}$. A delta $\mathrm{t}$ value for the camera of $3.5 \mathrm{~ms}$ is used for the fast tests and a value of $2.7 \mathrm{~ms}$ for the slow flight tests. This camera is positioned $2.18 \mathrm{~m}$ away from the laser sheet. The laser sheet is also purposely defocused to a width of about four $\mathrm{mm}$ in order to allow a greater illumination of particles moving in the out of plane direction, which is required by the magnitude of the axial flow [out of plane] 
through the core of the vortex. Figure 4.7 shows an action shot of the Night Vapor flying through the laser sheet.

\subsection{Wind Tunnel Experimental Setup}

The University of Dayton's low speed wind tunnel serves as a test bed to investigate the lift force measured on an aircraft with its wingtip located in close proximity to a wall. This wind tunnel has a Hartzell fan and a $60 \mathrm{Hp}$ motor that can produce a 90,000 CFM flow. The motor also can run at 1800 RPMs and a full length photo of the tunnel assembly can be seen in Figure 4.8. An eight pound six component sting force balance is located in an Aerolab test section and has a motor to move the balance through angles of attack from $-5^{0}$ to $25^{0}$ as commanded through the Aerolab computer program. The six components of the sting force balance include the roll moment, forward normal force, rear normal force, forward side force, rear side force, and axial force.

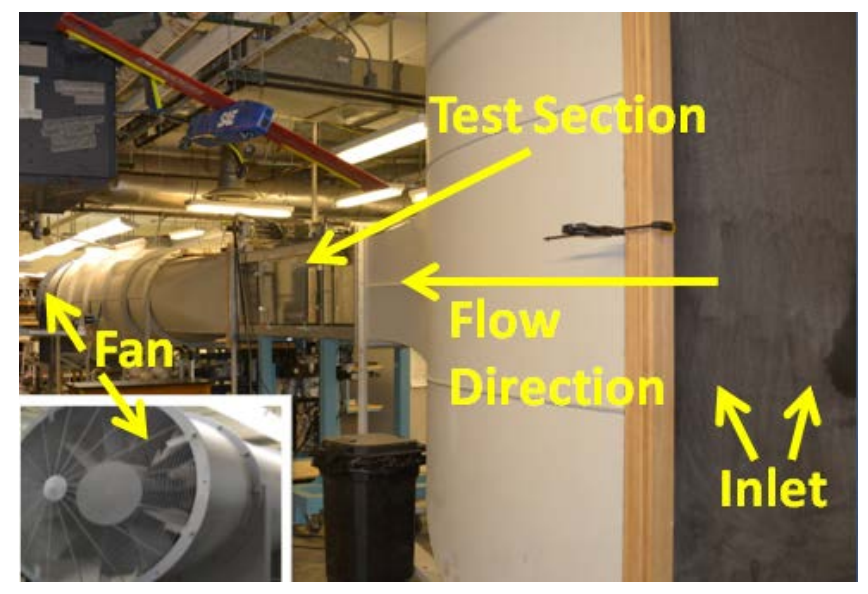

Figure 4.8: UD low speed wind tunnel. Close up image of fan inserted for reference. 
Preliminary testing is necessary in order to determine if the UD low speed wind tunnel can indeed provide accurate results when mounting the Night Vapor in the tunnel and simulating the free flight experiments. The average free flight fast speed of the Night Vapor is $2.96 \mathrm{~m} / \mathrm{s}(9.72 \mathrm{ft} / \mathrm{s})$ and the average free flight slow speed is $1.56 \mathrm{~m} / \mathrm{s}(5.11 \mathrm{ft} / \mathrm{s})$. The eight pound sting balance is tested to see if it can accurately provide lift and drag measurements for the 21 gram Night Vapor. As will be discussed in the results section, the UD low speed wind tunnel is not designed to perform testing for such a light aircraft at such low speeds, and the results are not consistent enough to accurately simulate the Night Vapor's free flight conditions. Thus, the goal of the wind tunnel testing shifts to quantifying the aerodynamic effects of the proximity of a wall on an airfoil shape instead of exclusively simulating the same flight speed and aircraft used in the PIV free flight tests. This decision is acceptable since the fluid dynamics of the situation are most important and not limited to the free flight PIV test flight conditions. As is discussed in the background research section of this thesis, a paper by Delisi [17] discusses the lack of Reynolds number sensitivity in the free vortex propagation. With this in mind, a heavier $\mathrm{RC}$ aircraft, the X-EC aircraft, is mounted to the sting balance and the wind tunnel runs at the highest dynamic pressure that the aircraft's structure can handle, $18.51 \mathrm{~m} / \mathrm{s}(60.74$ $\mathrm{ft} / \mathrm{s}$ ) for the fast flight tests. This procedure gives the highest possible measurements of lift and drag acting on the vehicle and it is expected that the wall effect on the vehicle will be clearly recognized. The experimental setup for the Night Vapor and X-EC airplanes is the same and thus only the X-EC wind tunnel setup will be described in detail. 
The X-EC airplane is mounted on the eight pound sting force balance through a rapid prototyped mount around a PVC pipe. This PVC pipe has a set screw attached which allows it to fasten securely onto the sting balance. This mount is then adhered with epoxy to a metal mount used to clamp the X-EC aircraft in place. See Figure 4.9 for a view of the X-EC attached to the sting force balance at a positive angle of attack. Figure 4.10 shows the location of a plexiglass wall that is attached to the roof of the wind tunnel. This wall helps to model the wall effects and Figure 4.11 shows a one $\mathrm{cm}$ distance between the wingtip and the wall. The wall has a $45^{\circ}$ bevel on the leading edge to cut down on the boundary layer of the flow on the plate. A top view of this leading edge clearly shows the bevel on the wall in Figure 4.12. This wall can translate and move from zero to seven or nine cm away from the wingtip, depending on the test condition, to measure the lift on the vehicle at different distances away from the wall. Tests are also performed without the wall present to serve as a comparison.

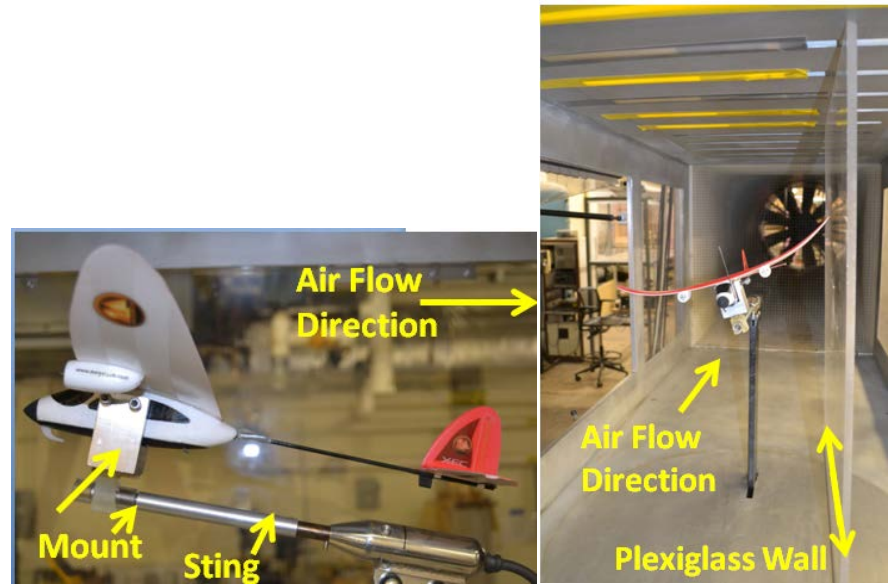

Figure 4.9: X-EC attached to the sting force balance.

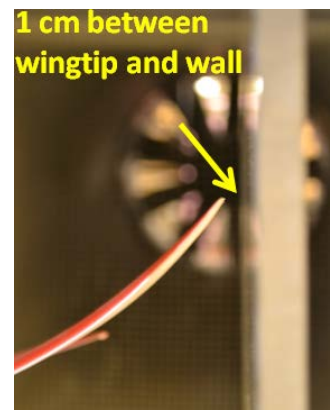

Figure 4.11: Figure 4.12:

Figure 4.10: Wall positioned in tunnel.
Top View

Flow Direction $45 \rightarrow$ Degree Bevel side
Wall one $\mathrm{cm}$ Top view of from tip. 
Figure 4.13 shows a more detailed image of the attachment designed for the interface between the Night Vapor and the X-EC aircraft and the sting. A set screw in the PVC pipe fastens the Night Vapor's mount to the sting force balance. Both aircraft are mounted at the quarter chord. Since the Night Vapor is a very stable aircraft, changing the location of the neutral point on the aircraft does not seem to affect the aircraft's stability. This is verified through changing the location of the Night Vapor's battery and hand launching the aircraft to see if it is still stable. The X-EC airplane is also mounted at the quarter chord for consistency. Figure 4.13 also shows the slot in the prototyped part that allows the set screw to pass through it in order to setup either aircraft for a roll angle. This helps to simulate a banked turning maneuver flight orientation as seen by the X-EC airplane in Figure 4.10. Figure 4.14 shows how the roll angle is measured when attaching either aircraft to the sting force balance. A carbon rod is attached perpendicularly to the aircraft and a level and protractor are used to measure the roll angle that the carbon rod reveals. A roll of $19^{0}$ is used since this is the average roll angle determined from the free flight tests.

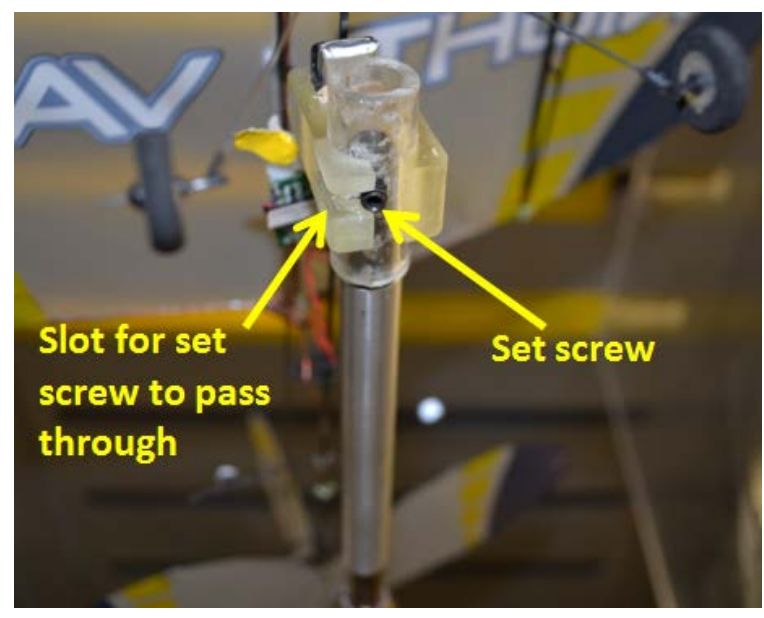

Figure 4.13: Interface between sting and aircraft mount for both Vapor and X-EC.

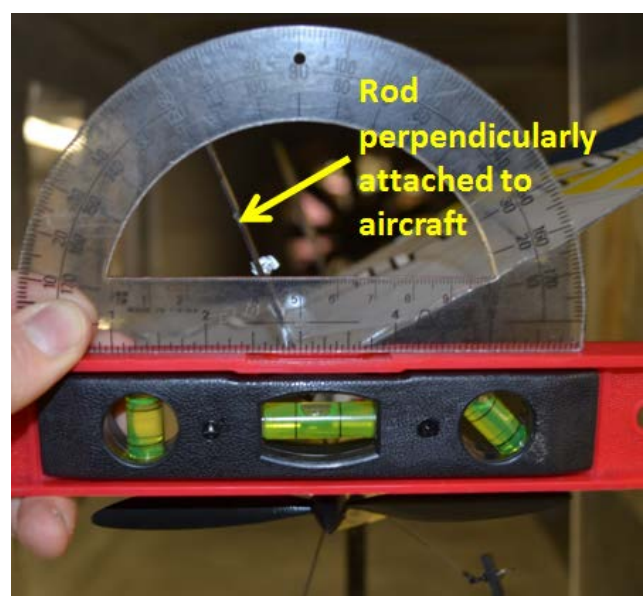

Figure 4.14: Method for measuring roll angle. 
A differential Ashcroft pressure transducer with a pressure range from -0.1 in of water column to 0.1 in of water column is used for the Night Vapor tests for the low speeds of 1.45 to $2.95 \mathrm{~m} / \mathrm{s}$ (5.11 to $9.72 \mathrm{ft} / \mathrm{s})$. For the X-EC airplane, an Omega differential pressure transducer is used for the 15.89 to $18.51 \mathrm{~m} / \mathrm{s}(52.12$ to $60.74 \mathrm{ft} / \mathrm{s})$ wind tunnel velocities. The Omega PX162-027D5V pressure transducer has a pressure range from zero to $6.9 \mathrm{kPa}$ (zero to one psi). The pressure transducer outputs a voltage signal to a multimeter and this voltage is converted to airspeed through using equations developed from a calibration process which was setup in the wind tunnel before this testing began.

Overall, the wind tunnel tests provide another method to analyze the lift of a MAV while flying in close proximity to a wall. Fast and slow, straight and turning, and wall and no wall tests are performed under conditions that will make it possible to analyze the basic fluid dynamic interactions between a wall and a wingtip. 


\section{CHAPTER 5}

\section{PROCEDURE}

\subsection{Proof of Concept PIV Procedure}

Two types of tests are performed in the PIV proof of concept testing period including fast straight flights along the wall as well as fast banked turning flights close to the wall. Three people are needed to run these tests.

- One person manually flies the Night Vapor.

- One person controls the PIV test as the Night Vapor flies past the wall.

- One person operates the Vicon motion capture system from the $\mu$ AVIARI control room.

Raw PIV images are collected as the Night Vapor flies through the field of view. These raw images are then passed through cross-correlation algorithms in the dPIV 2.0 software to produce velocity vector field plots. The image pairs are processed using two iterations, first a 64 and then a 32 pixel interrogation region with a 50\% overlap. The final vector image combines the multi-pass 64 and 32 pixel interrogation region vectors. A vector length cutoff filter is used to erase erroneous vectors with amplified lengths. No other filters are used. Finally, the vector plots are loaded into the visualization software, Tecplot 360, which will plot the vectors as a contour map and show the magnitudes of the vortex's velocity vectors as different colors. The z-vorticity of the Night Vapor's wingtip vortex is also plotted in Tecplot 360 as a contour plot. 


\subsection{Final PIV Testing Procedure}

\subsubsection{PIV Procedure}

A total of eight different types of tests are executed and the test matrix can be seen in Table 3.1 in the Theory behind Experiment section earlier in this thesis. The four tests listed in Table 3.1 are repeated without a wall present. These tests without the wall serve as a baseline for comparison against the tests performed with the wall present. The straight and level tests serve as a baseline of comparison against the turning maneuver tests. The high speed tests serve as a baseline of comparison against the slow speed, high angle of attack, tests. Only two people are needed to run these tests. One person operates the PIV equipment and checks the seeding quality while another person manually flies the Night Vapor and starts and stops the Vicon position recording program from a computer located in the flight room.

The raw PIV images are then passed through cross-correlation algorithms in the LaVision DaVis software to create the velocity vector fields. The image pairs are again processed with two iterations using a 64 and then a 32 pixel interrogation region with a $50 \%$ overlap. The final vector image combines the multi-pass 64 and 32 pixel interrogation region vectors. The multi-pass postprocessing routine in $\mathrm{DaVis}$ uses a median filter which can be configured to strongly remove the vectors if the difference compared to the median is greater than some factor (in the case of the experiments, 2) times the root mean square of the neighboring vectors. This filter also reinserts a vector if the difference compared to the median is less than three times the root mean square of the neighboring vectors. During the $64 \times 64$ to $32 \times 32$ interrogation region processing, an interpolation method is applied to fill empty spaces and a smoothing filter is also 
applied. These filters are chosen for this research since $\mathrm{DaVis}$ requires the user to apply these filters as a minimum form of filtering if the multi pass vector calculation postprocessing tool is used. A mask is also applied to the wall to rule out any noisy vectors that are produced at the wall. The DaVis software is used in this testing since it has more capabilities than the dPIV program; however the dPIV program is an excellent tool to use when first learning how to perform PIV. The vector plots are again loaded into Tecplot 360, which will plot the vectors as contour maps of velocity and z-vorticity.

\subsubsection{PIV Seeding Quality Validation}

Before collecting PIV data, it is necessary to verify that the PIV seeding is uniform and that the smoke particles scatter enough laser light in order for the crosscorrelation algorithms to work correctly. This verification process involves looking at the raw PIV images and checking the values of the intensity of the particles. Figure 5.1 shows an example of a full scale image of a raw PIV frame from the final PIV test setup. A haze of smoke particles can be seen. Figure 5.2 shows a zoomed in image of the same PIV frame as image 5.1. Zooming in allows the size of most of the smoke particles to be imaged as approximately two pixels. This is a desirable particle size as described earlier in this thesis. It is also advantageous for the smoke particles to have an intensity value of at least 400 counts above the background intensity for the cross-correlation algorithms to work correctly. This optimum value is obtained through collecting PIV images with various particle and background intensity differences and analyzing the quality of the data after processing. Figure 5.2 shows that most of the smoke particles have around 500 counts and that background has about 100 counts. Once it is established that the particle 
intensities look correct, it is time to tune the delta $\mathrm{t}$ time value between the PCO 1600 CCD camera frames.

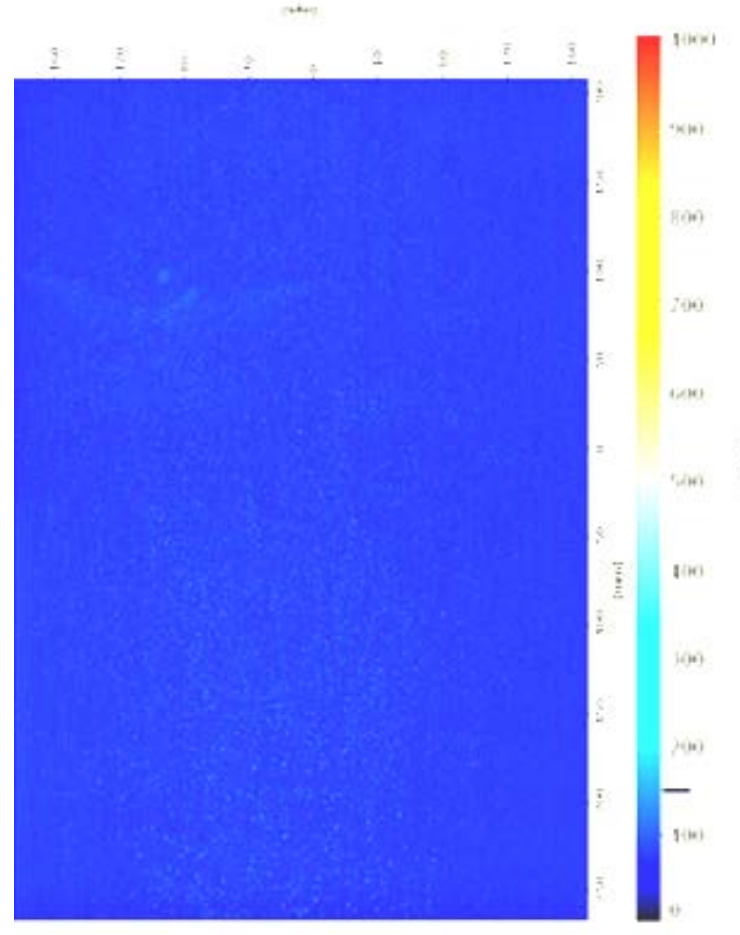

Figure 5.1: Full scale raw PIV image of the smoke particles within the field of view.

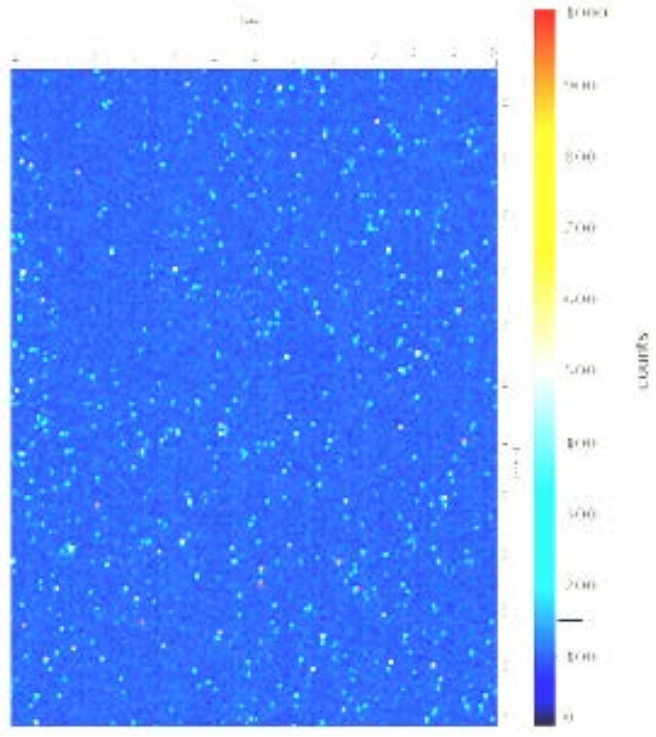

Figure 5.2: Zoomed in image of the smoke particles in Figure 5.1 .

The fast flight tests use a delta $\mathrm{t}$ time value of $3.5 \mathrm{~ms}$ while the slow flights used a delta $t$ value of $2.7 \mathrm{~ms}$. The theory behind tuning this time value involves calibrating the pixel displacements between the two image frames to be within a specific range as described in section 3.6.3 of this thesis. This section also describes that the ideal seed particle pixel displacement between the two frames for this research would be about eight to ten pixels given the 32 pixel final interrogation region size. The procedure to verify these pixel displacements for a test with a specified delta $t$ value involves processing the data in the $\mathrm{DaVis}$ software and exporting a plot showing the pixel displacements for the PIV images. Figure 5.3 shows a contour plot of the pixel displacements for a fast straight 
test without a wall. Both of the wingtip vortices can be seen and the area around the core of both vortices has the highest pixel displacement of around eight to ten pixels colored in yellow. The delta t value for this test is $3.5 \mathrm{~ms}$ and the pixel shift shows that this is the correct delta $t$ for this particular testing condition. Similar plots are made when determining the correct delta $t$ value for the slow velocity tests.

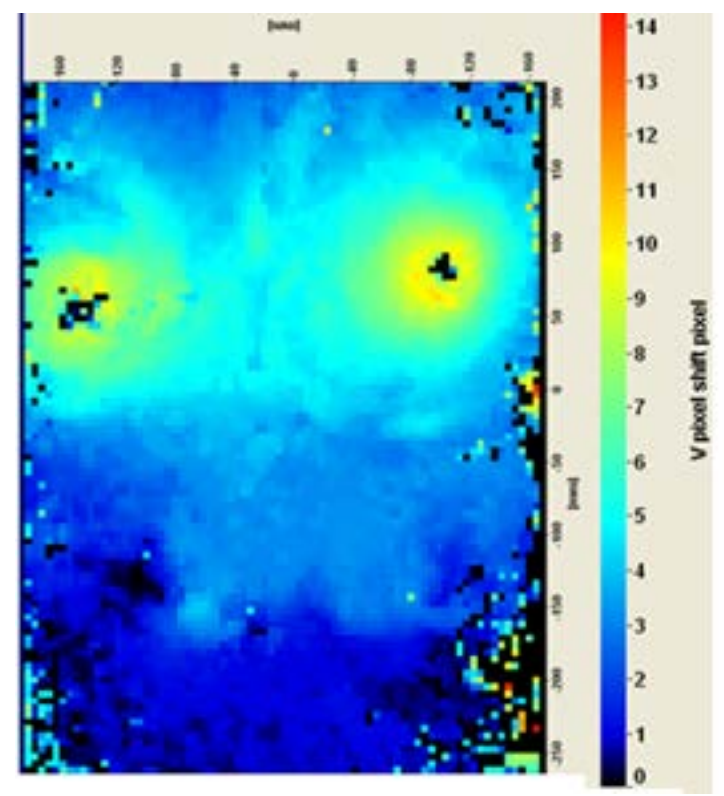

Figure 5.3: 8-10 ideal pixel shifts for wingtip vortices is achieved for a fast straight test without a wall.

\subsection{Vortex Identification}

Once the velocity contour plots of the wingtip vortices are produced in Tecplot 360 , the $\Gamma_{1}$ and $\Gamma_{2}$ vortex identification schemes described in the background theory section of this thesis are used to identify the core and boundary of the wingtip vortices. Before the values of $\Gamma_{1}$ and $\Gamma_{2}$ can be calculated, it is first necessary to extract the correct points to analyze in Tecplot 360 . These points are extracted from meshes created in Tecplot 360. The meshes are a collection of coordinate points defined over the PIV images. These coordinate points contain the $\mathrm{x}$ and $\mathrm{y}$ coordinate values of the points as 
well as the velocity and z-vorticity values of the PIV contour plots at those coordinate points. These meshes are needed for the $\Gamma_{1}$ and $\Gamma_{2}$ vortex identification algorithms. These algorithms perform calculations over each grid point in order to determine if that grid point is located in the core, boundary, or outside the vortex. The results of these calculations for each point in the mesh can be plotted over the entire vortex PIV image to clearly define the center and boundary of the vortex.

Determining the appropriate mesh spacing can be difficult because it is challenging to decide on the appropriate number of grid points needed to define the vortex and accurately perform the vortex identification calculations in a reasonable amount of time. If too few grid points are used, there will not be enough points located inside the core and boundary of the vortex to be used in the vortex identification calculations. Thus, these calculations will not be as accurate compared to calculations that use more points located within the vortex. If too many grid points are used, the computational time necessary to run the Matlab vortex identification algorithms increases and time will not be available to process as many images. The correct mesh size which balances the amount of grid points located within the vortex with limited computational time is a delta $\mathrm{x}$ and delta y mesh spacing of $4.77 \mathrm{~mm}$ between grid points.

Several meshes are necessary for the vortex identification algorithms since these equations, 3.1 and 3.2, would be undefined in the automated Matlab code if one mesh is used to define both the grid of points for the core of the vortex as well as the grid of points forming the location of the velocity vectors around the core. Figure 5.4 is a repeated image of Figure 3.1 for convenience. Point $\mathrm{P}$ is the point under consideration to be the center of the vortex and point $\mathrm{M}$ is the location of the velocity vector $\mathrm{U}_{\mathrm{M}}$. If $\mathrm{P}$ 
equals $M$ then the vector PM is 0 and this will make the denominator of the $\Gamma_{1}$ vortex center and $\Gamma_{2}$ vortex boundary, equations 3.1 and 3.2, go to zero and make the entire equation undefined. This creates a problem in the automated Matlab computer code using these equations. Thus, in order to make the automated Matlab code run without this undefined term, a mesh of M points is created in Tecplot 360 over the entire PIV image and a second mesh of $\mathrm{P}$ points is created. The mesh of $\mathrm{P}$ points includes grid points located the midpoint of each cell in the $\mathrm{M}$ mesh. Figure 5.5 shows a $\mathrm{Z}$-vorticity contour plot of a wingtip vortex near a wall from a straight and fast test. This first image does not have the meshes $\mathrm{M}$ or P displayed. Although they are very fine and hard to see, Figure 5.6 shows both meshes $\mathrm{M}$ and $\mathrm{P}$ displayed over the entire contour plot.

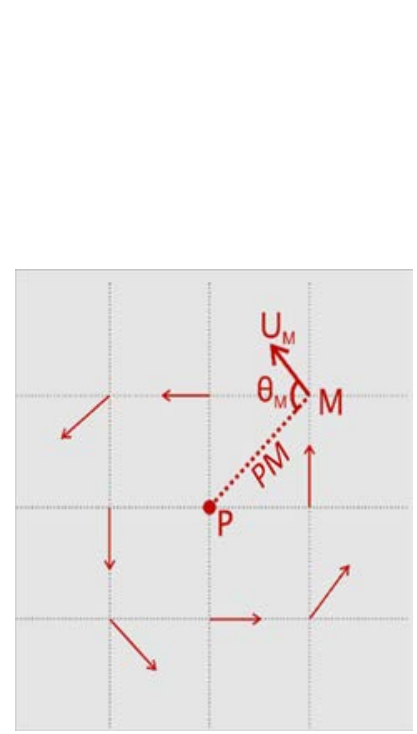

Figure 5.4: Location of variables used in the $\Gamma_{1}$ vortex core identification method [32].

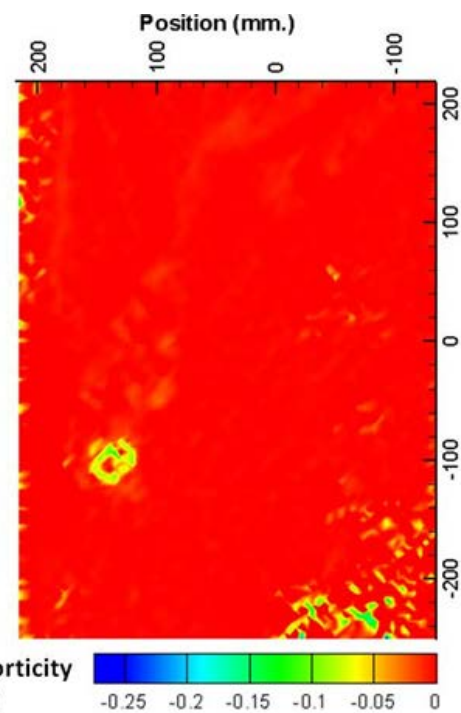

Figure 5.5: Z-vorticity contour plot of a wingtip vortex for a fast straight flight near the wall.

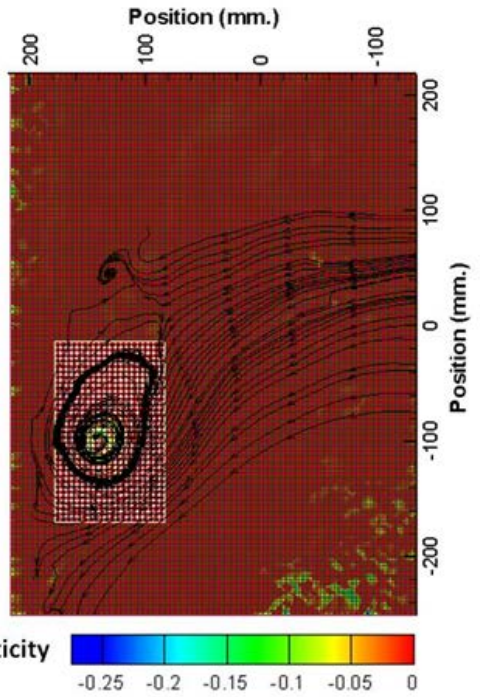

Figure 5.6: Streamlines plotted to help determine the size of the Msub mesh.

One limitation of the $\Gamma_{1}$ and $\Gamma_{2}$ vortex center and boundary identification approach is that it will calculate the center and boundary of multiple vortices if several are present in the data. In order to clearly apply these methods to the vortex of interest 
only and avoid including the boundary of any secondary vortices present from the second wingtip vortex, two smaller meshes derived from the entire $\mathrm{M}$ and $\mathrm{P}$ meshes are defined. The $\Gamma_{1}$ and $\Gamma_{2}$ calculations are then performed over these smaller M and P meshes. In order to do this, it is necessary to extract data points around the wingtip vortex of interest into subzones in Tecplot 360 . The M points surrounding the vortex are extracted into the small rectangular white subzone seen in Figure 5.6. This subzone is called the Msub mesh because it extracts its data from the corresponding points in the original $\mathrm{M}$ mesh. The size of the Msub mesh is unique to each test depending on the size and shape of the wingtip vortex. The size of Msub is decided upon through plotting streamlines of the vortex and ensuring that the dimensions of the subzone include the area of the vortex where the streamlines recirculate around the vortex. The white Msub mesh can be seen in Figure 5.6 along with the recirculating streamlines located within this Msub mesh. Next, it is necessary to create a subzone of $\mathrm{P}$ points, called Psub, located in the midpoint of each cell of the Msub mesh. The actual values of this Psub mesh are extracted from the corresponding points in the original P mesh. A zoomed in image of the white Msub mesh and the black Psub mesh can be seen in Figure 5.7. Once these subzones are created, the $\mathrm{x}, \mathrm{y}, \mathrm{V}_{\mathrm{x}}$, and $\mathrm{V}_{\mathrm{y}}$ values from each sub mesh are exported and loaded into a Matlab code which performs the $\Gamma_{1}$ and $\Gamma_{2}$ calculation over each point in the Msub and Psub meshes. Using these smaller meshes also decreases the computational time for the automated Matlab code to run. 


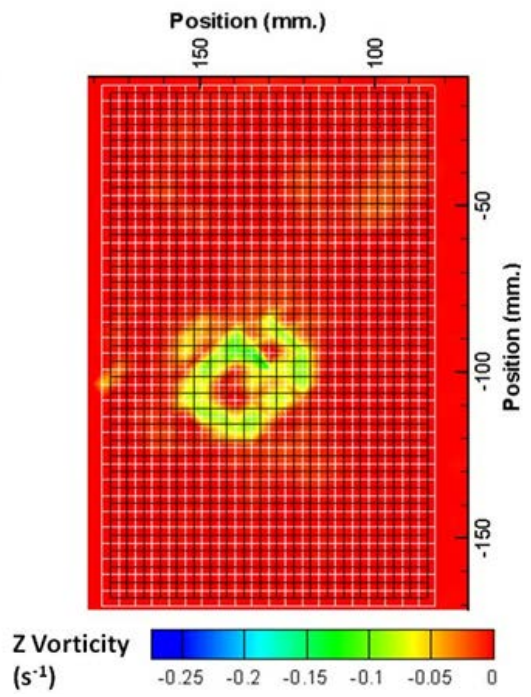

Figure 5.7: Zoomed in image of white Msub mesh and black Psub mesh.

One additional note includes the fact that the $\Gamma_{2}$ equation, equation 3.2 , is undefined for points where the $\mathrm{x}$ and $\mathrm{y}$ component velocities of points $\mathrm{P}$ and $\mathrm{M}$ are both zero. This happens because the magnitude of the difference in these velocities is located in the denominator of this equation. In order to make the automated Matlab code run correctly, any grid points in the Msub or Psub meshes that contain zero values for $\mathrm{u}$ and $\mathrm{v}$ velocity are removed from the data sets loaded into the computer code. These points are not considered for the vortex identification code since they would not produce points that would be defined as the vortex core or boundary via the $\Gamma_{1}$ and $\Gamma_{2}$ vortex identification equations, 3.1 and 3.2 .

The automated vortex identification Matlab code outputs the $\mathrm{x}$ and $\mathrm{y}$ coordinate values within the Psub mesh where the corresponding $\Gamma_{1}$ value is within the range of 0.85 to one. Graftieaux et al [31] state that the magnitude of $\Gamma_{1}$ "typically" has a value from 0.9 to one for a point to be located within the core of a vortex. It is decided that the extended lower boundary for the $\Gamma_{1}$ calculation is acceptable since the lower bound of the region is only reduced by $5.56 \%$. This extended boundary helps to identify the core of 
the vortex since it is discovered that some of the PIV images do not contain a $\Gamma_{1}$ value that is higher than 0.9. However, it is easy to find $\Gamma_{1}$ values that are higher than 0.85 . The Matlab code also outputs the $\mathrm{x}$ and $\mathrm{y}$ coordinate values within the Psub mesh that have a $\Gamma_{2}$ value within the range of 0.55 to 0.75 . In his paper, Graftieaux et al [31] state that the bounds for $\Gamma_{2}$ are "typically" within the range of 0.6 to 0.7 . It is decided that it is acceptable to reduce the lower bound by $8.33 \%$ and increase the upper bound by $7.14 \%$ since Graftieaux et al [31] stated that these bounds are "typical bounds". The PIV images used in this thesis research are not of ideal quality due to imperfect seeding, as has already been discussed in Chapter four section 4.2. The relaxed $\Gamma_{2}$ values give significantly better vortex boundary results that show the boundary of the entire vortex. Using the bounds that Graftieaux et al [31] propose resulted in vortex bounds that are sparse and not clearly defined. The reasoning behind the "typical" bounds provided by Graftieaux et al [31] are described in section 3.2 of this thesis. The $\mathrm{x}$ and $\mathrm{y}$ coordinate values for the vortex center $\Gamma_{1}$ and vortex boundary $\Gamma_{2}$ are then loaded into Tecplot 360 . The vortex center point is plotted as a black $\mathrm{X}$ in the z-vorticity magnitude plot in Figure 5.8a for a wingtip vortex produced from a fast straight flight test near the wall. Figure 5.8a also shows that the points that form the boundary of the vortex are plotted as yellow stars $(*)$. The vortex center is also seen by a red $\mathrm{X}$ in the normalized velocity contour plot in Figure 5.8b. In this figure, the points that form the boundary of the vortex are again plotted as yellow stars. The vortex center in these two plots is the maximum $\Gamma_{1}$ value. However it is also possible to plot all of the $\Gamma_{1}$ values obtained within the boundaries described earlier in order to clearly see the size of the entire vortex core, as seen in Figure 5.8c. 


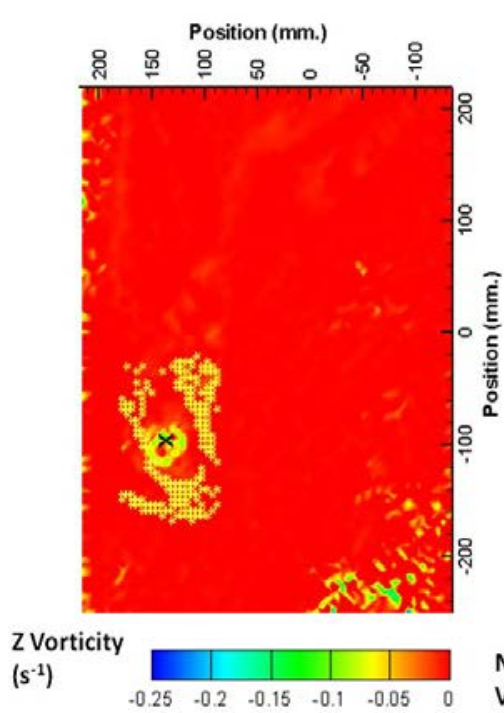

a.

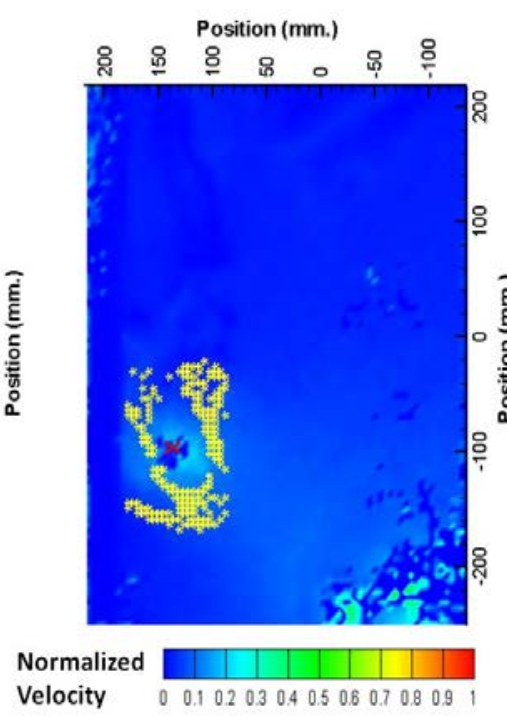

b.

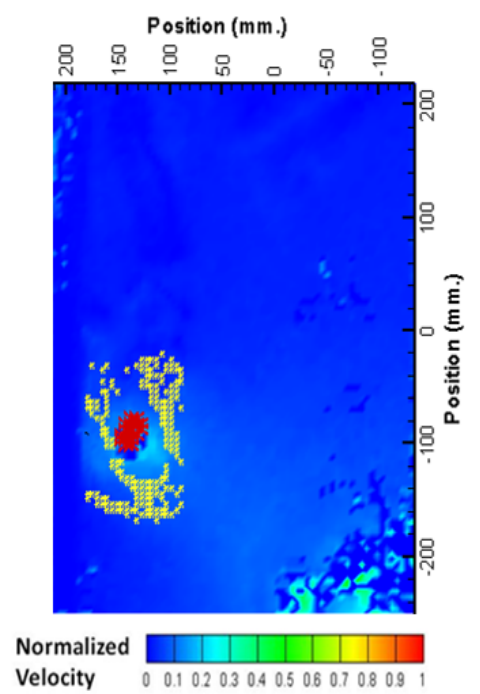

c.

Figure 5.8: Vorticity magnitude and normalized velocity contour plots showing the vortex center and vortex boundaries.

\subsection{Calculating Vortex Circulation}

\subsubsection{Area Integral over the Z-Vorticity}

As is described in section 3.3 of this thesis, one way to calculate the circulation of a vortex, $\Gamma_{z}$, involves taking the integral of the $z$-vorticity, $\omega_{z}$, over the area of a vortex, dS. See Equation 3.3. In order to perform this calculation in Tecplot 360 an area to integrate over must be defined. Figure 5.9 shows a multitude of boxes centered on a zvorticity contour plot of a wingtip vortex produced as a result of flying a fast straight flight test near the wall. Many boxes are drawn in order to complete a sensitivity analysis for quantifying how much the area integration circulation value changes as a function of the size of the area integration box. Area integration circulation calculations are made for each box. The results from this sensitivity analysis will be discussed in detail with the rest of the results in Chapter six of this thesis, but it is discovered that the area integration 
circulation calculation is significantly sensitive to the integration area chosen. This sensitivity analysis is performed before the $\Gamma_{1}$ and $\Gamma_{2}$ vortex identification algorithms are applied to this research. The results of this sensitivity analysis show the need for a standardized method to define the core and the bounds of the vortex with which to integrate over. Figure 5.10 shows a zoomed in image of the same z-vorticity contour plot from Figure 5.9. Only one area integration circulation box exists and is centered on the vortex core point from $\Gamma_{1}$ and limited to the boundaries of the $\Gamma_{2}$ yellow stars.

The area integration method used to calculate circulation in Tecplot 360 is validated and deemed first order accurate. The area integration circulation calculation process in Tecplot 360 is validated by applying the method to a simply integrated vorticity function and then comparing the circulation result to that of the closed form integrated solution. This same approach is also used to show that the circulation is still accurate if the area integration box used is not a square shape, but more of a rectangular shape. 


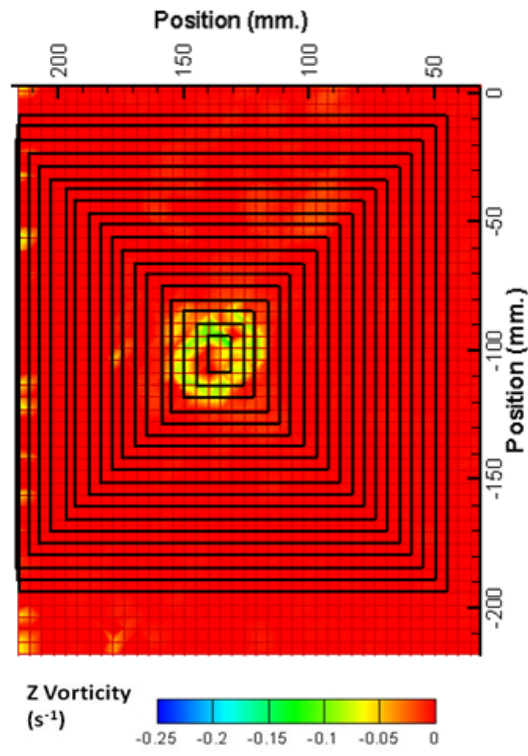

Figure 5.9: Multitude of area integration boxes for circulation calculation sensitivity analysis.

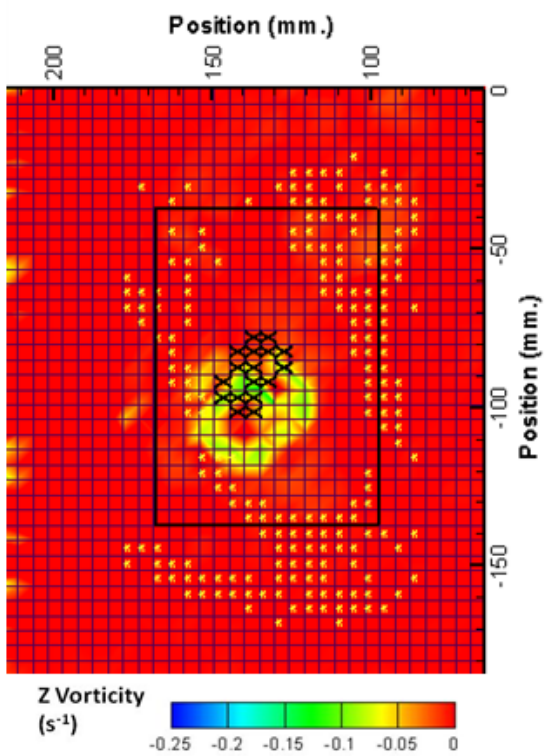

Figure 5.10: Integration box for area integration circulation calculation.

\subsubsection{Line Integral of the Velocity over a Closed Curve}

In section 3.3 of this thesis, another method used to calculate the circulation of a vortex, $\Gamma_{\mathrm{z}}$, is discussed. This method involves calculating the line integral of the velocity, $\mathrm{V}$, over the closed curve, dl, of the vortex. See Equation 3.4. Figure 5.11 shows a green ring encompassing the same example wingtip vortex from a fast straight flight test past the wall. This green ring is developed through extracting points along a streamline and creating a closed curve used for this circulation line integration. This streamline is chosen for this test since it represents the highest area of recirculating streamlines as seen in Figure 5.6. This streamline is used for the line integration since it has many other streamlines around it that follow nearly the same path. These streamlines with nearly the same path can be seen in Figure 5.6 as a black circle of many streamlines in close proximity to one another. Since the streamlines follow nearly the same path, this means that the velocity vectors that form these streamlines are located very close together and 
point in the same direction. Thus, these velocity vectors are very similar to one another and the flow is more consistent in those locations. This streamline is also located within the area integration boxes previously described and is centered on the $\Gamma_{1}$ vortex center shown by the red X's in Figure 5.11. This streamline also matches the $\Gamma_{2}$ vortex boundary shown by the yellow stars in Figure 5.11. A term line in Tecplot 360 can be used to trim the streamline down to just one revolution around the vortex. Even though this trimmed streamline recirculates in a tight radius compared to the other streamlines in the image, the streamline is still not technically a closed curve since it spirals out with a slowly increasing radius. The location of the term line shows the discontinuity between the start and end of the streamline. See Figure 5.12. Linear interpolation between the first two and last two points along the streamline helps to close the curve for the line integration circulation calculation. See Figure 5.13.

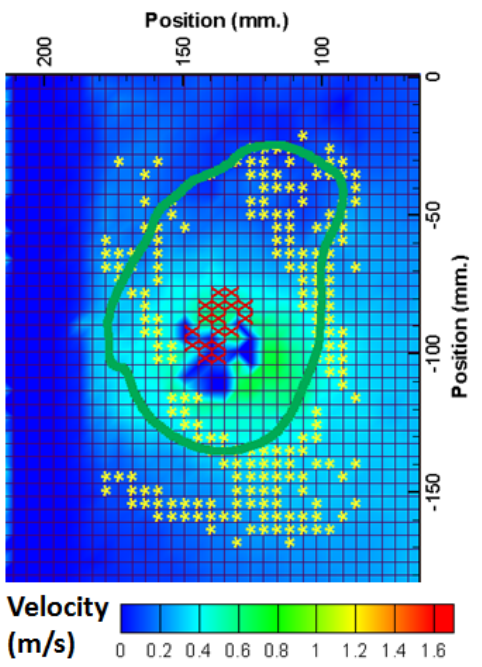

Figure 5.11: Streamline that is used for the line integration circulation calculation.

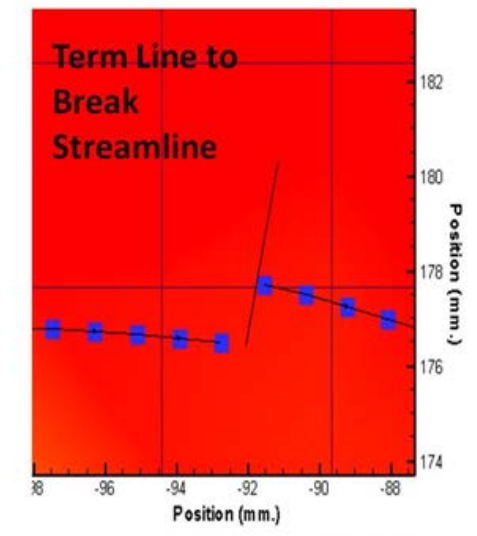

Z Vorticity $\left(s^{-1}\right)$

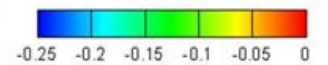

Figure 5.12: Discontinuity in beginning and termination of a streamline.

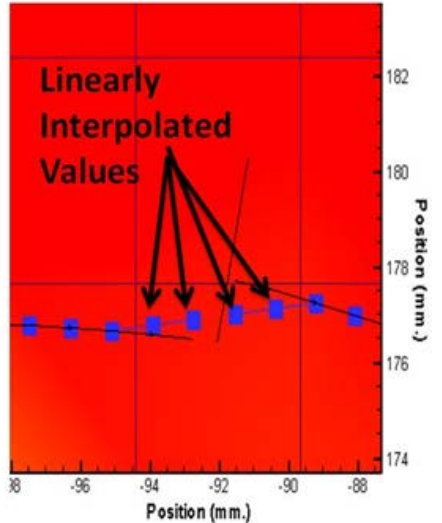

Z Vorticity $\left(\mathrm{s}^{-1}\right)$

Figure 5.13: Linear interpolation helps the first two and last two points line up with the rest of the streamline. 
In order to obtain a ballpark estimation for the wingtip vortex circulation, it is possible to calculate the estimated circulation for a straight and level flight wingtip vortex. This estimated circulation is calculated through using the Kutta-Joukowski theorem. See Equation 3.5. The estimated circulation for a straight and level flight wingtip vortex has a value of $0.16 \mathrm{~m}^{2} / \mathrm{s}$. Since this is a straight and level flight test, the lift of the aircraft is assumed to be equal to the weight of the aircraft. The wingspan, $b$, is used in this calculation instead of the distance between the two wingtip vortices since the distance between the vortices changes slightly across the tests depending on which image in the wake is analyzed.

Table 5.1: Values used in wingtip vortex circulation estimation.

\begin{tabular}{|c|c|}
\hline Wingtip Vortex Circulation Estimation for Free Flight Fast Flight Speed \\
\hline Humid Density of Air $\left(\mathrm{kg} / \mathrm{m}^{\wedge} 3\right)$ & 1.16 \\
\hline Fast Velocity $(\mathrm{m} / \mathrm{s})$ & 2.96 \\
\hline Lift $(\mathrm{N})=$ Weight & 0.21 \\
\hline $\begin{array}{c}\mathrm{b}=\text { Wingspan used to standardize distance between } \\
\text { vortices }(\mathrm{m} .)\end{array}$ & 0.37 \\
\hline Circulation $\left(\mathrm{m}^{\wedge} 2 / \mathrm{s}\right)$ & 0.16 \\
\hline
\end{tabular}

Once the circulation is calculated in Tecplot 360 it is then possible to use the Kutta-Joukowski theorem to calculate the lift of the wingtip vortex. Calculating the circulation and the lift from the PIV images of the wingtip vortices will help to quantify the effect of the wall on the wingtip vortex. Knowing how the wall affects the wingtip vortex will give insight into how the aerodynamics of a MAV are affected while flying near a wall and how the controls of the vehicle may need to change to compensate for the wall effect. In order for MAV missions in the urban environment to succeed, MAVs 
must be able to adapt their flight controls to account for the wall effect and this is the primary focus of this thesis. While the circulation calculation represents one method that can be used to quantify the effect of the wall on the wingtip vortex and the MAV flight controls, measuring the elliptical shape of the vortex an also help to investigate this phenomenon.

\subsection{Measuring Wingtip Vortex Ellipticity}

The effect of the wall on the wingtip vortex can also be quantified by measuring the circular or elliptical shape of the wingtip vortex bounds. This in turn will help to determine how a MAV's flight controls must be changed in order to accurately fly along a wall. The yellow star points in Figures 5.14 and 5.15 show the bounds of the vortex calculated from the $\Gamma_{2}$ criteria and the black X's show all the points that form the core of the vortex as determined from the $\Gamma_{1}$ criteria. Figure 5.14 shows the circular shape of the wingtip vortex for a fast, straight flight test without the wall present. The length of the light blue or purple lines in this figure represent the measured circular diameter of the boundary of the vortex while the green line represents the diameter of the core of the vortex. Figure 5.15 is a wingtip vortex from a slow turning test along the wall. The blue vertical line represents the major axis length of the elliptical shape of the vortex bound while the purple horizontal line represents the minor axis length of the vortex bound. The green line represents the diameter of the vortex core. The length of all of these lines is computed by using the inspect tool in Tecplot 360 to obtain the $\mathrm{x}$ and $\mathrm{y}$ coordinate values of the ends of each of the lines. Then, the distance formula is used to measure the length between the two points. 


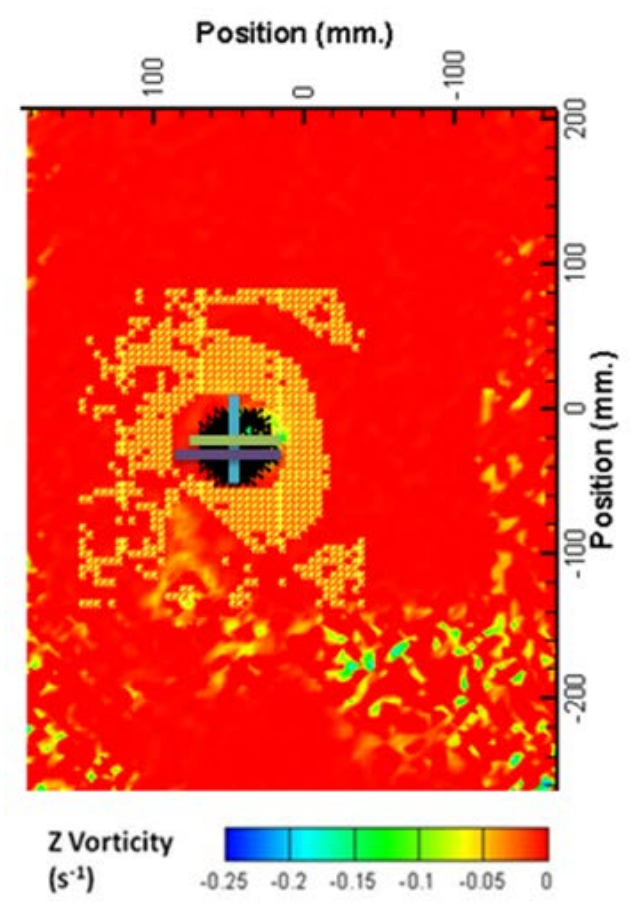

Figure 5.14: Circular vortex from a fast straight test without wall present.

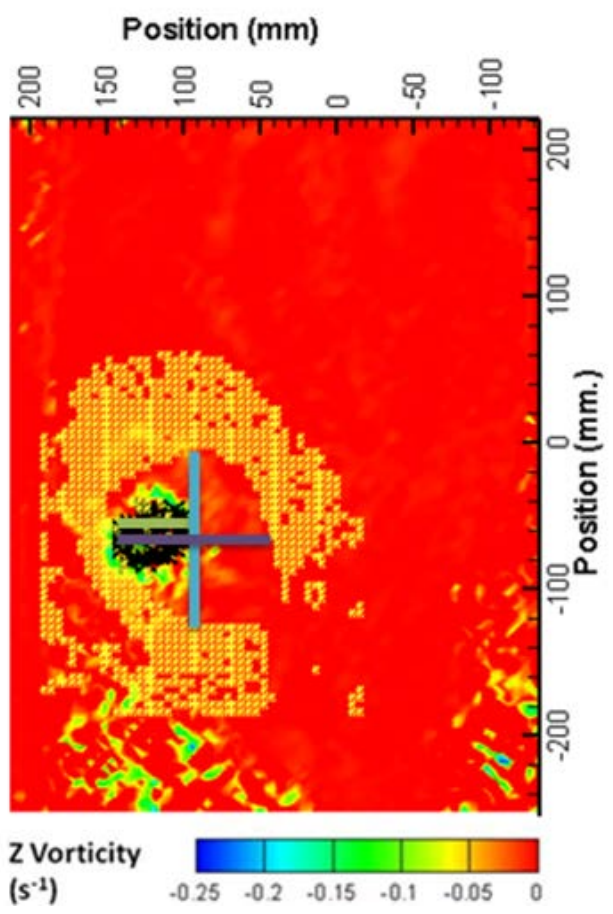

Figure 5.15: Elliptical vortex from a slow turning wall test.

The measured diameter of the core of the vortex is used later to normalize the vortex circulation value in an attempt to provide a more level field for comparison while the vortex boundary dimensions are used to calculate the ellipticity. Ellipticity is defined as the length of the major axis of the ellipse divided by the length of the minor axis of the ellipse as shown in equation 5.1. As described earlier in this thesis, measuring the circular or elliptical shape of the wingtip vortices helps to locate the instabilities that develop in a wingtip vortex as a result of flying along a wall.

$$
\text { Ellipticity }=\frac{\text { length of major ellipse axis }}{\text { length of minor ellipse axis }}
$$

Once the ellipticity values are calculated and compared for the various tests, it is then possible to see the effect of the wall on the shape and stability of the wingtip vortex. 
This is investigated by comparing the consistency of the wingtip vortex circulation values for the tests completed with or without a wall present. This is completed by taking the three best tests from each of the eight free flight test conditions and calculating the circulation through using the area and line integration techniques for areas and streamlines located within the $\Gamma_{2}$ vortex boundary. The criteria for determining which images are the best include looking at the resolution of the vortex in the PIV images as well as the distance between the vortex and the wall for the tests conducted near the wall. A sensitivity analysis based on choosing different area sizes for the area integration circulation calculation is also conducted and the circulation values recorded. All of these circulation values for the three best tests from each test condition are then compared based on the consistency of the values. These circulation values are also related to the shape and stability of the wingtip vortex to determine the effect of the wall on the vortex.

\subsection{Data Analysis Procedure for Vicon Data}

An improvement within the procedure for this testing includes placing Vicon markers at the entrance and exit of the PIV room to help track when the Night Vapor passes through the room within the Vicon data. This makes it easy to identify the average velocity of the Night Vapor for all the tests as it passes between these two points. A 20 point moving average is used to average the velocity values to help smooth the data. A value of 20 points is chosen since it is the minimum number of points that is consistently needed to help smooth the data. The reference locations of the Vicon markers also make it possible to determine when the aircraft flies through the laser sheet within the Vicon data. This is advantageous because then the Night Vapor's angle of 
attack and roll angle from the Vicon data can be determined when it passes through the laser sheet.

\subsection{Wind Tunnel Testing Procedure}

The tests described in the test matrix in Table 3.1 are conducted in the UD low speed wind tunnel for cases with and without a wall present. For the wall tests, data is collected when the wall is positioned from one to eight $\mathrm{cm}$ away from the wingtip. As is discussed in the experimental setup section of this thesis, the UD low speed wind tunnel is not designed to collect accurate measurements for the slow flight speed and low weight of the Night Vapor. Therefore, the X-EC RC airplane is implemented into the test instead. For both the Night Vapor and the X-EC RC airplane, the process for running the wind tunnel experiment is the same.

First, the aircraft is mounted on the sting balance and a tare measurement is recorded. The pressure transducer is also calibrated to read zero pressure. Next, the wind tunnel is turned on and the pressure transducer output is recorded to calculate the velocity of the flow and three data sets are collected. Then the tunnel is turned off and another set of tare data is collected after the tunnel is allowed to settle for five minutes. A five minute settling time is used since the pressure transducer registered zero flow velocity after five minutes. When processing the data, the before and after test tare normal force measurements are averaged and subtracted from the normal force average calculated across all of the tests in order to zero out any bias error. This tare process is also repeated for the axial force as well as the side force for the banked turning tests. Alpha sweep tests are performed for the Night Vapor and data is taken at one degree increments from 
negative six to 14 degrees to help decide if the data for the $C_{L}$ vs. alpha curve for the Night Vapor are the correct order of magnitude and if the equipment is accurate enough.

\subsubsection{Night Vapor Wind Tunnel Testing Procedure: Knowledge Gained}

First, an alpha sweep for the Night Vapor is performed from 14 to negative six degrees with reference to the Night Vapor's wing. This test is performed in order to relate the $\mathrm{C}_{\mathrm{L}}$ of the aircraft to the angle of attack of the aircraft. Once an equation is developed to relate these variables, the wind tunnel angles of attack can be compared to the angles of attack calculated with the Vicon data through knowing the $\mathrm{C}_{\mathrm{L}}$ for both tests. For the wind tunnel test, the Night Vapor is mounted on the force balance with a negative ten degree angle of attack in order to prevent the aircraft's tail from interfering with the force balance. The Night Vapor's wing has a four degree angle of incidence compared to the fuselage and thus the aircraft's wing is actually at a negative six degree angle of attack when the fuselage and force balance are parallel to the floor of the wind tunnel. This explains why the alpha sweep angle of the wing goes beyond the negative five to 25 degrees allowed by the motion system in the wind tunnel. Alpha sweep tests are conducted for the full Night Vapor, a second night vapor with the rudder and elevator removed, and the Night Vapor with the propeller on or off. The rudder and elevator are removed in an attempt to make it easier to mount the Night Vapor without using the negative ten degree angle of attack mount. However, the alpha sweep results did not look realistic and thus this approach is abandoned. The tests with the propeller on one quarter, half, three quarters, or full throttle also did not provide accurate results because the motor could not keep up with the wind tunnel as the battery drained. As a result, the drag 
values are higher for the tests with the propeller on. Therefore, it is decided that using the entire Night Vapor aircraft with the propeller off yields the best results. The results of the alpha sweep $C_{L}$ vs. alpha plot can be seen in Figure 5.16. The blue experimental wind tunnel lift curve line is compared with a theoretical lift curve line for a finite wing. Good agreement is seen between the two curves and the slope of the linear portion of these curves is also very similar.

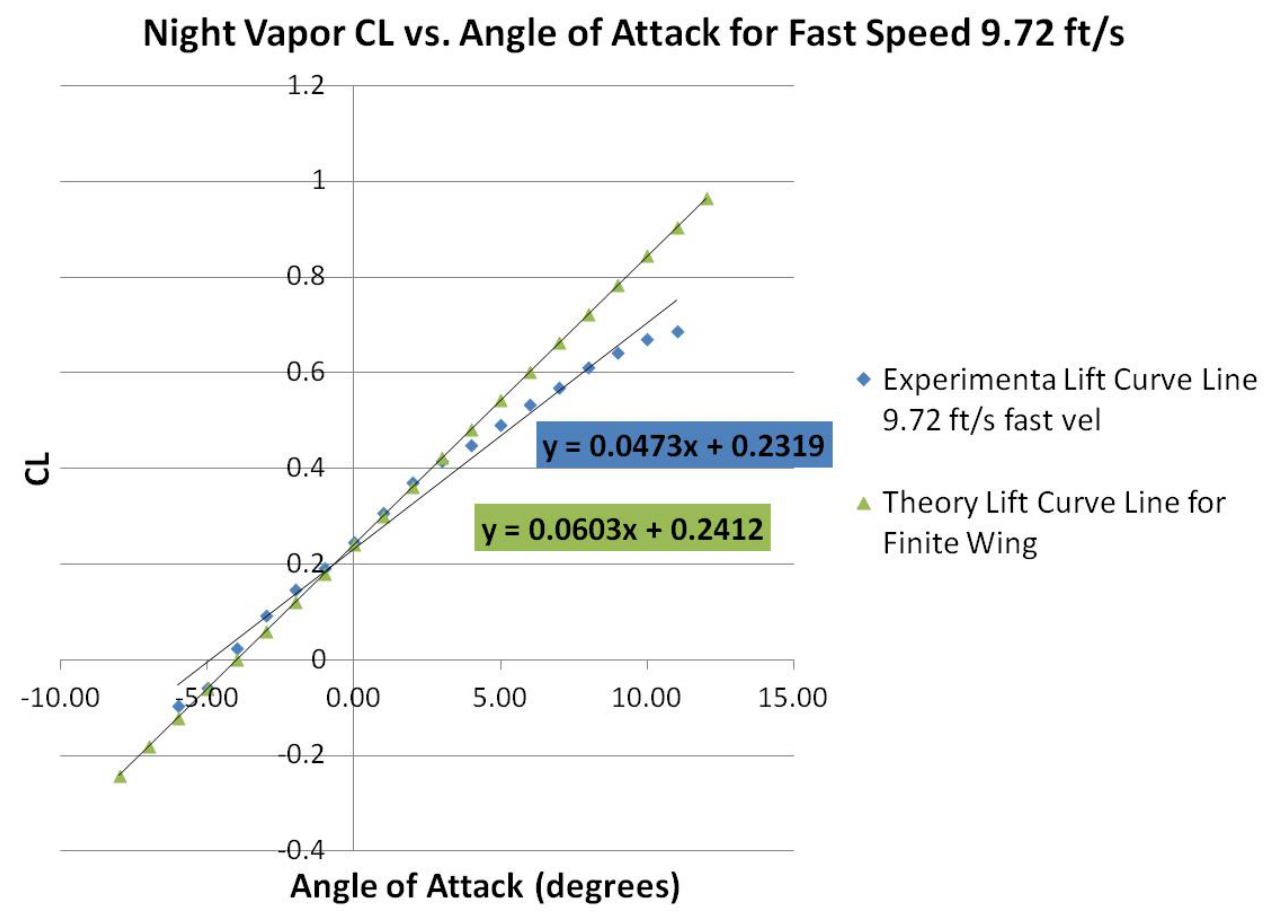

Figure 5.16: $C_{L}$ vs. alpha curves and lift curve slope equations for the experimental and theoretical data.

The theoretical wing lift curve slope for a finite wing, equation 5.2 [44], is determined for the Night Vapor in equations 5.3 and 5.4.

$\mathrm{e}=$ Oswald efficiency factor, assume a value of 0.9 because it is a reasonable value. $\mathrm{AR}=$ aspect ratio of Night Vapor $=2.74$

$C_{L \alpha 2 D \text { airfoil }}=$ Lift curve slope of a symmetric two-dimensional airfoil with a $2 \pi$ lift curve slope. 


$$
\begin{gathered}
C_{L \alpha 3 D \text { wing }}=\frac{C_{L \alpha 2 D \text { airfoil }}}{1+\frac{C_{L \alpha 2 D \text { airfoil }}}{\frac{\pi e A R}{1}}} \\
C_{L \alpha 3 D \text { wing }}=\frac{\frac{2 \pi}{\text { radian }}}{1+\frac{\frac{2 \pi}{\text { radian }}}{\pi(0.9)(2.74)}} \\
C_{L \alpha 3 D \text { wing }}=\frac{0.0603}{\text { degree }}
\end{gathered}
$$

The result in equation 5.4 is then used to plot the theoretical line in Figure 5.16 with the assumption that the zero-lift point occurs at negative four degrees. This zero-lift point is decided to have a value of negative four degrees through calculating the maximum camber of the Night Vapor's wing and then comparing this maximum camber to that of many airfoils in an online airfoil database. This database is called the Airfoil Investigation Database and specifically relates to RC aircraft specifications [45]. The Night Vapor's wing has a maximum camber of 9\%. Most of the airfoils in the database that have a similar maximum camber value compared to the Night Vapor have a zero-lift angle of negative two to negative four degrees. Thus, it is assumed that the Night Vapor has a zero-lift angle of negative four degrees. This aids with determining the theoretical curve in the plot in Figure 5.16.

It is also possible to assume that the lift of the aircraft is equal to the weight of the aircraft and use equation 5.5 to calculate the $\mathrm{C}_{\mathrm{L}}$ of the Night Vapor from the free flight PIV tests based on the flight velocity from each test. 


$$
C_{L}=\frac{L}{\frac{1}{2} \rho V^{2} A}
$$

Once the equation of the line is known for the Night Vapor wind tunnel alpha sweep data in Figure 5.16, it is then possible to use the resulting $C_{L}$ calculated for each PIV free flight test from equation 5.5 in equation 5.6 to calculate the angle of attack of the Vapor for each free flight test. Once the average angle of attack from the free flight tests is known, this same angle of attack can be implemented into the wind tunnel tests. The results of the Night Vapor wind tunnel testing and why the results are not consistent will be discussed in detail in Chapter six of this thesis.

$$
\alpha=\frac{C L-0.2319}{0.0473}
$$

\subsubsection{Wind Tunnel Data Processing Procedure}

The processing of the wind tunnel data for the Night Vapor and the X-EC airplane is completed as follows. Figure 5.17 shows a side view of the free body diagram of the sting force balance at a specified angle of attack, alpha. The sting balance is the orange element in the figure. The variables are defined below and the equations used to calculate the lift and drag for the sting at a prescribed angle of attack is listed in equations 5.7- 5.12. 


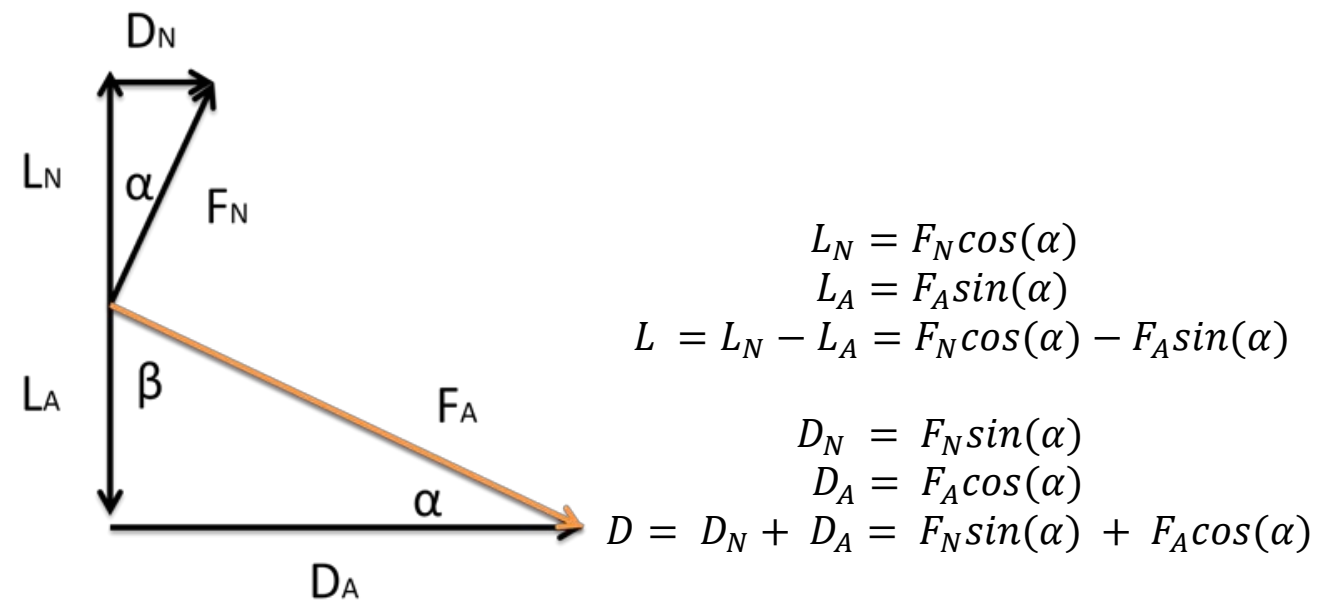

Figure 5.17: Free body diagram of the sting force balance as viewed from the side.

$\mathrm{L}_{\mathrm{N}}=\mathrm{Lift}$ due to the normal force

$\mathrm{L}_{\mathrm{A}}=$ Lift due to axial force

$\mathrm{F}_{\mathrm{N}}=$ Normal force from sting - average tare normal force

$\mathrm{F}_{\mathrm{A}}=$ Axial force from sting - average tare axial force

$\mathrm{D}_{\mathrm{N}}=$ Drag due to normal force

$\mathrm{D}_{\mathrm{A}}=$ Drag due to axial force

$L=$ Total lift force

$D=$ Total drag force

If the airplane is mounted at a bank angle for a turning test, the side force from the sting force balance must also be taken into account when calculating the total lift on the aircraft. Figure 5.18 shows a head on view of the bank angle of an airplane's wing when mounted on the sting balance. The red line represents the wing at a bank angle of phi. The variables used in this free body diagram are described below. 


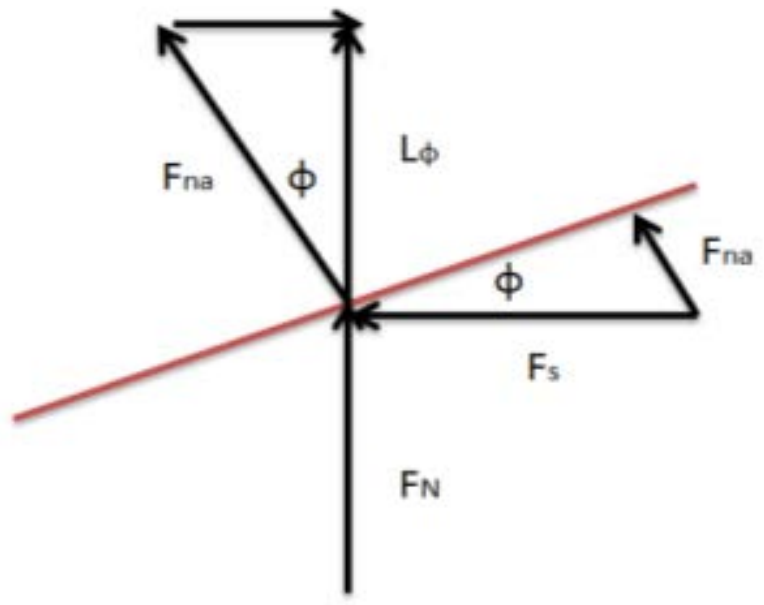

$$
\begin{aligned}
& F_{n a}=F_{s} \sin (\phi) \\
& L_{\phi}=F_{n a} \cos (\phi)
\end{aligned}
$$

Figure 5.18: Free body diagram of the airplane wing (red) as viewed from head on.

$\mathrm{F}_{\mathrm{N}}=$ Normal force from balance

$\mathrm{Fs}=$ Side force

$\mathrm{F}_{\mathrm{na}}=$ Component of side force that is normal to the aircraft

$\varphi=$ Roll angle

$\mathrm{L} \varphi=$ Component of the side force that is normal to the balance

The average side force is determined through subtracting the tare side force from the side force for each individual test and averaging the result. Once the component of the side force that is normal to the balance is calculated, equation 5.14, it is added to the overall average normal force recorded from the balance. Then this total normal force is used in equation 5.7 and 5.10 to calculate the lift and drag respectively due to the normal force. Then equations 5.9 and 5.12 can be used to calculate the total lift and drag on the aircraft. Once these values are known, the coefficient of lift and drag can be calculated through using equations 5.5 and 5.15 respectively.

$$
C_{D}=\frac{D}{\frac{1}{2} \rho V^{2} A}
$$




\subsubsection{Uncertainty Analysis}

In order to determine how accurate the wind tunnel data will be, it is necessary to perform an uncertainty analysis. First, the accuracy of the force balance motion system is performed. The force balance motion system moves the sting force balance to its extreme angle of attack positions of 24 and negative five degrees. The extreme angles are used in order to provide representative uncertainties across the spectrum of angles that are possible with the motion system. At each location, five measurements of the force balance angle of attack are made through using a handheld digital angle measuring device. The average angle across the five measurements is calculated. The standard deviation across all five measurements is also calculated. The standard deviation is then divided by the square root of the number of measurements taken at that angle of attack. The resulting value is the standard deviation of the mean. This value is rounded to one significant figure and demonstrates the accuracy of the motion system and how much above or below the desired angle of attack the aircraft may be located. This process yields the following uncertainty. The maximum angle of attack of the motion system is $24 \pm 0.06$ degrees while the minimum angle of attack of the motion system is $-5 \pm 0.03$ degrees.

In order to determine the uncertainty associated with the pressure transducer used to calculate the velocity of the wind tunnel, the manufacturer's datasheet is referenced to obtain the hysteresis and repeatability percentage of full scale values associated with this instrument. The procedure to calculate the total pressure transducer error is seen below in Table 5.2. The percentage of full scale values are multiplied by the actual full scale measurement range of the pressure transducer to obtain the error value for the hysteresis 
and repeatability errors. Then, the total error for the pressure transducer after calibration is found by taking the square root of the sum of the squares of the error values for the hysteresis and repeatability errors.

Table 5.2: Omega pressure transducer error analysis.

\begin{tabular}{|c|c|c|c|}
\hline \multicolumn{4}{|c|}{ Omega Pressure Transducer Error Analysis } \\
\hline Type & $+-\%$ of Full Scale & Full Scale of Gauge (psf) & + - Error Value (psf) \\
\hline Hysteresis & 0.25 & 144 & 0.36 \\
\hline Repeatability & 0.25 & 144 & 0.36 \\
\hline Total Error after Calibration & \multicolumn{3}{|c|}{0.51} \\
\hline
\end{tabular}




\section{CHAPTER 6}

\section{RESULTS}

\subsection{Introduction}

The theory behind the research in this thesis is based on the fact that a vortex has an influence on a body or flow in its environment as a function of the distance between the vortex and this body or flow. The wingtip vortex of a MAV and its distance away from the wall could have an aerodynamic effect on the MAV. Free flight PIV experiments and wind tunnel testing are used in an effort to investigate how the aerodynamic interactions between a wingtip vortex and a wall affect the controls of a MAV.

\subsection{PIV Free Flight Testing}

The PIV free flight testing helps to collect data on how a MAV's wingtip vortex interacts with a wall and how the MAV's flight controls are affected as a result of this wingtip vortex interaction with a wall. Significant improvements are made within the PIV testing method between the proof of concept testing and the final PIV free flight testing. Figure 6.1 shows an example of one of the best velocity vector field plots that is 
obtained from the proof of concept testing for a fast turning maneuver near a wall. Two vortices can be seen among the multitude of noisy vectors. Even through processing this test with a vector length cutoff filter in order to remove some of the noisy vectors, this image still shows many noisy velocity vectors. In comparison, Figure 6.2 shows a velocity vector field plot from the final PIV testing for a fast turning test near the wall. The $\mathrm{DaVis}$ software is used in the final testing since it has more capabilities than the dPIV program used in the proof of concept testing. Figure 6.2 shows that the two wingtip vortices are clearly defined and do not contain many noisy vectors compared to Figure 6.1. This degree of improvement is a result of the three weeks of testing time that is dedicated to improving the method of creating a uniformly dispersed smoke field. Having uniform seeding is imperative in order to produce accurate PIV images that will help to investigate how the wingtip vortex interacts with the wall and affects the flight controls of the MAV. 


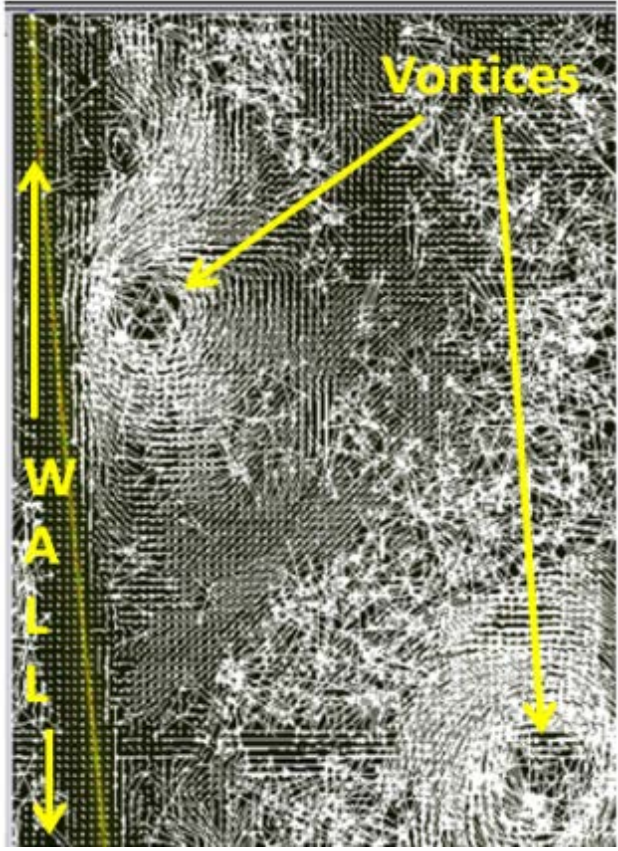

Figure 6.1: Noisy velocity vectors are seen in the proof of concept PIV testing.

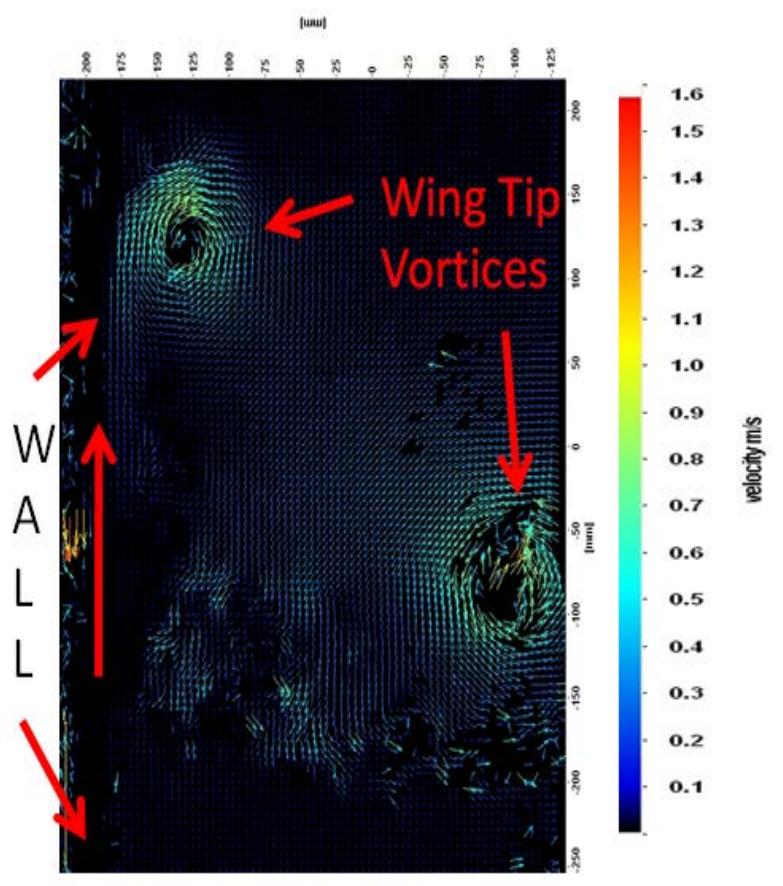

Figure 6.2: Final PIV velocity vector field shows clear vortices with few noisy vectors compared to Figure 6.1 .

\subsubsection{Vortex Identification Results}

The vortex identification methods that are described in section 5.3 and visualized in Figures 5.8 a through $5.8 \mathrm{c}$ and Figures 5.14 and 5.15, result in a clear description of the vortex core size as well as the size of the boundary of the entire vortex. This robust approach takes out the ambiguity and subjective view of where the boundaries of the vortex are located. These boundaries are needed in order to develop a repeatable and accurate method of determining the size of the vortex to include in the area and line integration circulation calculations. Knowing the boundary of the wingtip vortex core as well as the outer edge boundary of the entire vortex also helps to identify evidence of elliptical instabilities as a result of the wall deforming the wingtip vortex. All of this 
analysis will help to achieve the final goal of determining how the interaction of the wingtip vortex and the wall affect the flight controls of the MAV when flying near the wall.

\subsubsection{Wingtip Vortex Circulation Results}

The circulation of the PIV wingtip vortices is calculated for tests with and without a wall present with the intent of discovering a significant difference in circulation due to the wall interacting with a wingtip vortex. A change in circulation of the wingtip vortex due to the wall could affect the MAV's flight controls. For the area integration approach, a sensitivity analysis is performed in order to examine how the size of the area used affects the circulation value. Several attempts at normalizing this circulation by the size of the integration box used or the distance between the vortex core and the wall are also investigated. However, little difference in circulation is noticed for the tests conducted with or without the wall present. For this reason, the details about the multiple methods used to normalize the vortex circulation values are discussed in Appendix A. On the other hand, discernible trends still exist and the images included here represent the most meaningful information gathered from the variety of circulation calculation attempts. These meaningful trends are defined as being significant to this research since they provide the most insight into how the circulation shows the wingtip vortex interaction with a wall and how this wall effect can affect MAV flight controls.

Figure 5.9 in Chapter five of this thesis shows an example of the multitude of area integration boxes used for the area integration circulation calculation sensitivity analysis. Figure 6.3 shows the resulting raw circulation values associated with the area integration 
circulation calculation sensitivity analysis for a fast straight test conducted with and without the wall present. While it is difficult to notice a distinct difference in the circulation values between the tests with and without a wall present, the general trend of the increasing circulation with an increasing area of integration can be noticed. While both the tests with and without the wall demonstrate this trend, the fact that circulation increases with increasing area can still relate to how the wingtip vortex interacts with a wall and affects the MAV's flight controls.

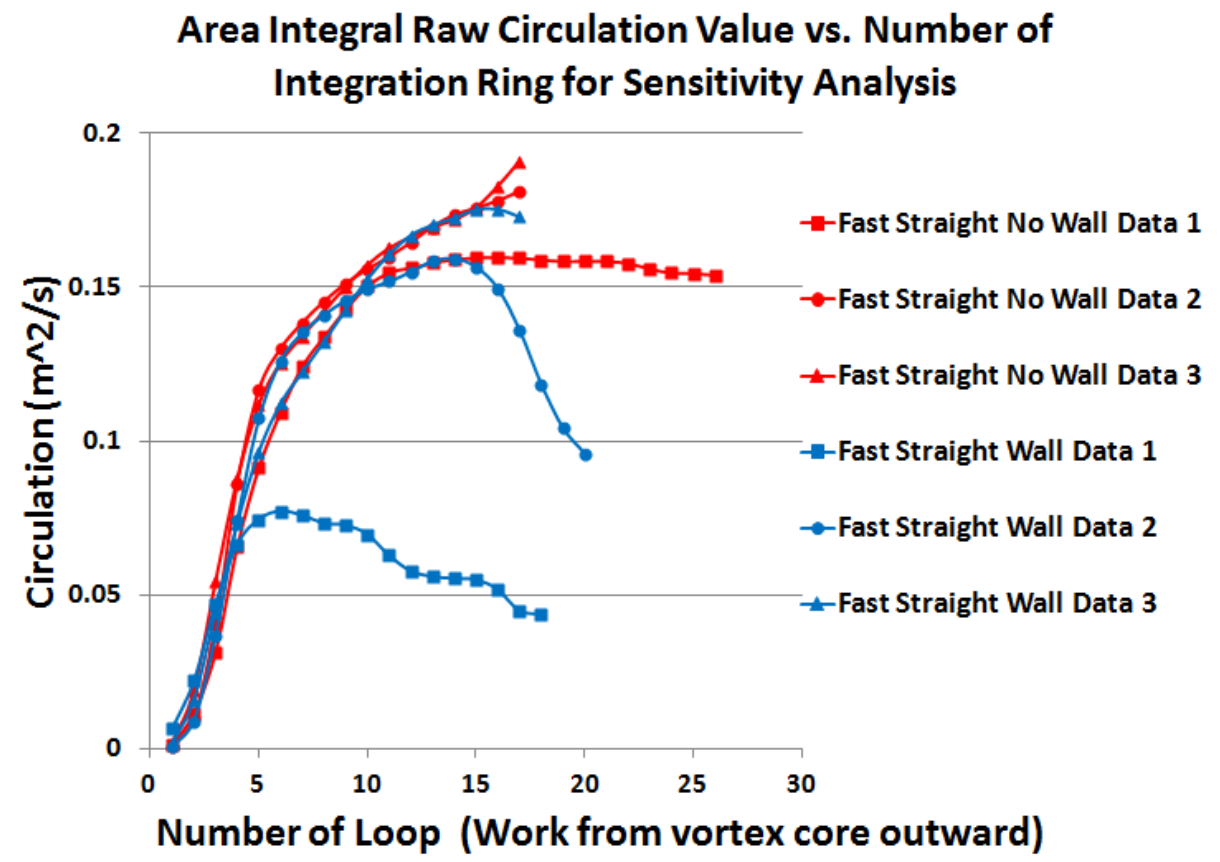

Figure 6.3: This area integration circulation sensitivity analysis plot shows a trend of increasing circulation values with increasing integration area until the circulation becomes relatively constant for a large area.

In their paper, Baik et al [33] also perform a similar sensitivity analysis as discussed in section 3.3 of this thesis. While Baik et al [33] perform this circulation sensitivity analysis over multiple vortices in the field of integration, this trend in circulation rise can still apply to the research of this thesis. Figure 3.5 shows that as the area used in the circulation calculation increases (see Figure 3.6), the circulation values 
also rise. This general trend is also seen in Figure 6.3 for the tests conducted with and without a wall present. In order to see this trend more accurately, the increase in circulation between individual areas is calculated as a percentage of the total increase in circulation for the whole sensitivity analysis. This data is calculated for the results from Baik et al [33] as well as the fast straight free flight PIV tests with and without the wall present. This analysis focuses on three areas where circulation rapidly increases and does not focus on areas where the circulation is relatively constant. Table 6.1 shows the increase in circulation between three individual areas as a percentage of the total increase in circulation for the whole sensitivity analysis. It can be seen that as more of the leading edge vortex core is included in the research from Baik et al [33], the percentage increase of circulation rises. Similar trends are seen for the three areas under consideration in this thesis research and thus the increasing area leads to larger circulation values. This trend relates to MAV flight controls since the circulation of the wingtip vortex flow increases the farther away from the wingtip vortex core the flow is located within the vortex boundaries. 
Table 6.1: Percentage rise in circulation focused on three areas around the vortex where the circulation increased the most.

\begin{tabular}{|c|c|c|c|}
\hline \multicolumn{3}{|c|}{ Sensitvity Analysis: Rise in Circulation Between Areas Chosen as Percentage of Total } \\
Rise in Circulation \\
\hline $\begin{array}{c}\text { Area of Vortex Included } \\
\text { in Integration }\end{array}$ & Baik et al [33] & Fast Straight Without Wall & Fast Straight Wall \\
\hline $\begin{array}{c}\text { Area Including only small } \\
\text { amount of vortex core }\end{array}$ & $15 \%$ & $13 \%$ & $5 \%$ \\
\hline $\begin{array}{c}\text { Area including only the } \\
\text { entire vortex core }\end{array}$ & $37 \%$ & $22 \%$ & $17 \%$ \\
\hline $\begin{array}{c}\text { Area including vortex } \\
\text { core and a small area } \\
\text { outside vortex boundary }\end{array}$ & $37 \%$ & $16 \%$ & $24 \%$ \\
\hline
\end{tabular}

In an attempt to understand why there is not a distinguishable difference in circulation between the PIV wingtip vortices for tests with and without a wall present, several attempts at normalizing the circulation are implemented. The most intriguing normalization attempt involves using the area integration circulation sensitivity analysis information since circulation values are found for different areas around the vortex. The circulation of each box is normalized by the circulation of the wingtip vortex core and the radius of the box is normalized by the vortex core radius. The circulation of the wingtip vortex core for each test is found through performing the area integral within the bounds of the $\Gamma_{1}$ vortex core boundary points. An example of the results of this normalization process is seen in Figure 6.4 for fast straight flights with and without the wall present. The $\mathrm{x}$ axis is plotted on a logarithmic scale to help focus on the circulation values between the two black vertical lines. The first black vertical line with the square symbol represents the $\Gamma_{1}$ boundary of the vortex core. The second black vertical line with the triangle symbol represents the $\Gamma_{2}$ outer vortex boundary. These bounds help to focus the 
area of interest of this large plot into a narrow area. Within the bounds of the vortex, the normalized circulation continuously rises with the increasing normalized vortex radius. This trend is seen for the tests conducted with or without the wall present. While the circulation of the tests with the wall seems to rise faster than the tests without the wall for this specific group of tests, this trend is not consistent amongst other tests. Overall this trend verifies the same trend seen in the research performed by Baik et al [33]. This trend relates to MAV flight controls since the circulation of the wingtip vortex flow increases the farther away from the wingtip vortex core the flow is located within the vortex boundaries.

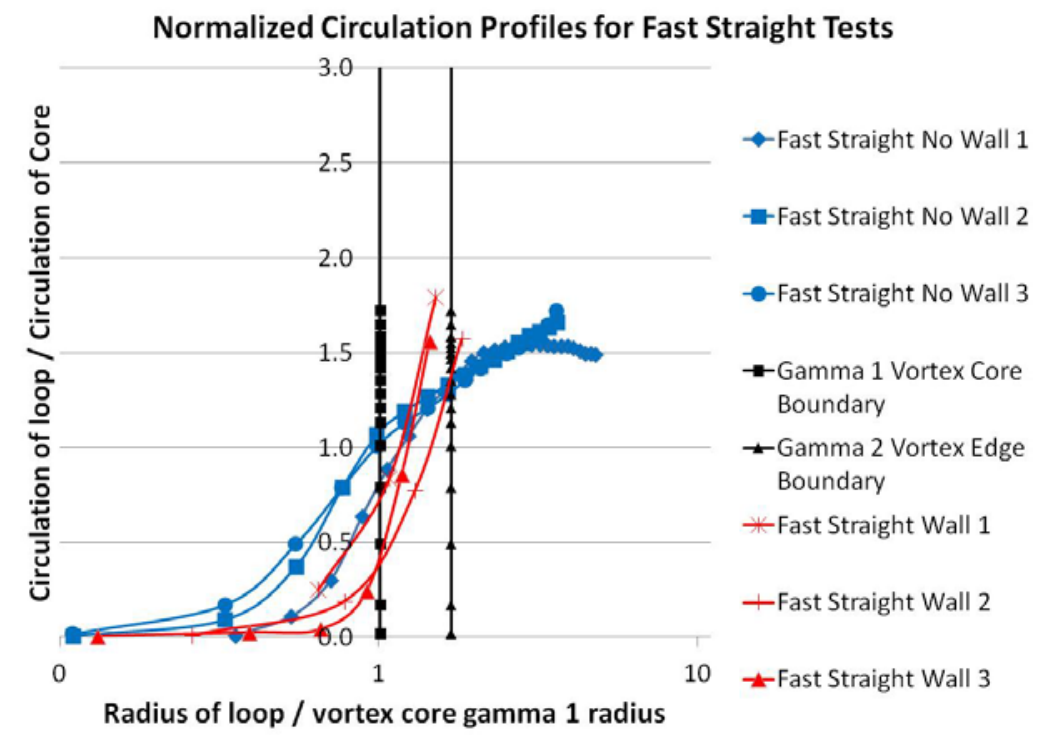

Figure 6.4: This normalized area integration circulation sensitivity analysis shows that the normalized circulation increases with the constantly increasing normalized radial position within the vortex boundaries.

This normalization process is chosen because a paper by Birch [46] shows that this process provides this clear trend of constantly increasing normalized circulation with constantly increasing normalized radial length for trailing vortices. Within the vortex bounds of this thesis research, the normalized circulation values and trends in Figure 6.4 
resemble the normalized circulation values and trends seen in the literature in Figure 6.5. Since it is not possible to average PIV tests in this research, sporadic z-vorticity surrounding a vortex easily contributes to errors in the area integration circulation sensitivity analysis. For this reason, the area integration boxes used in Figure 6.4 are limited to the areas that do not contain noisy z-vorticity. If the boxes with noisy zvorticity are used, the normalized circulation values become unreasonably high compared to the literature in Figure 6.5. This process of eliminating integration boxes that contain noisy z-vorticity in the area integration circulation sensitivity analysis is repeated for each individual test in this thesis research to ensure the accuracy of the data. Images showing examples of the noisy sporadic z-vorticity and how it impacts the normalized circulation values can be seen in Appendix A. Again, the ability to average multiple PIV images would help to minimize the errors in circulation calculations due to inconsistencies located at the edge of the vortices. Overall, this trend of increasing normalized circulation with increasing normalized radial position is important in the wall effects research in this thesis since a MAV's flight controls relate to the circulation around the wingtip vortex. 


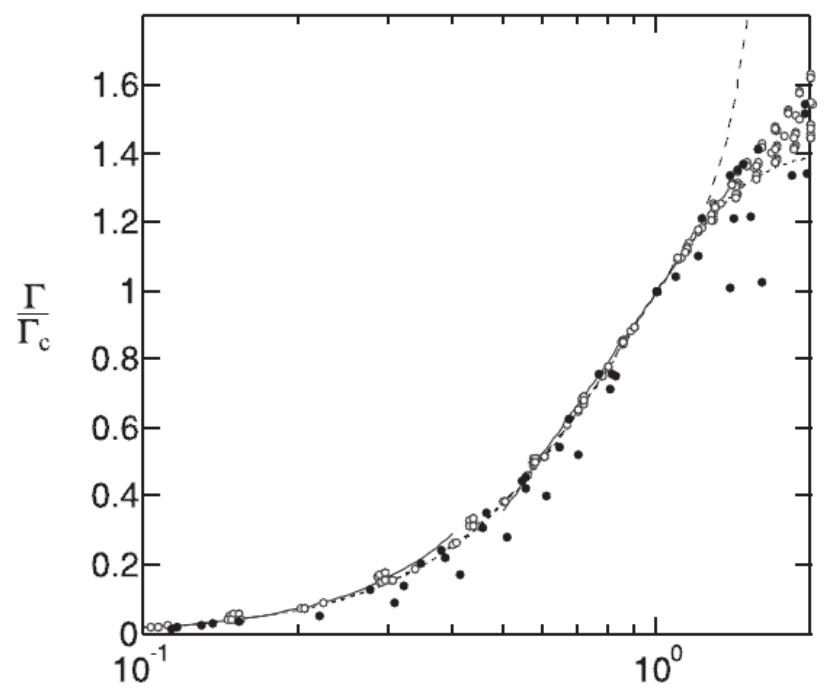

Figure 6.5: The trend of constantly increasing normalized circulation with constantly increasing normalized radial position for trailing vortices can be seen in this plot [46].

For the PIV wall effects line and area circulation integration calculations performed within the $\Gamma_{2}$ vortex boundary, both methods of calculating circulation yield similar results. Figure 6.6 shows the average raw circulation values for the tests conducted with and without the wall present for all eight test conditions. While the results of a few individual test conditions do not match up perfectly, such as the slow turn test without the wall, most of the corresponding line and area integration circulation values are close to one another. The slow tests always have a higher circulation compared to corresponding fast test. According to the Kutta-Joukowsi theorem (equation 3.5), if the flight velocity goes down, then it is expected that the circulation will rise in order for the airplane to have enough lift to maintain flight. This result is important for a slow flying MAV because it may need to adjust its flight controls when flying near a wall since the circulation of the wingtip vortex increases due to the slow flight velocity. 
The results in Figure 6.6 are also validated by Baik et al [33] in Figure 3.5. This plot shows that the line integration and area integration circulation calculations provide similar circulation values when applied to the same vortex.

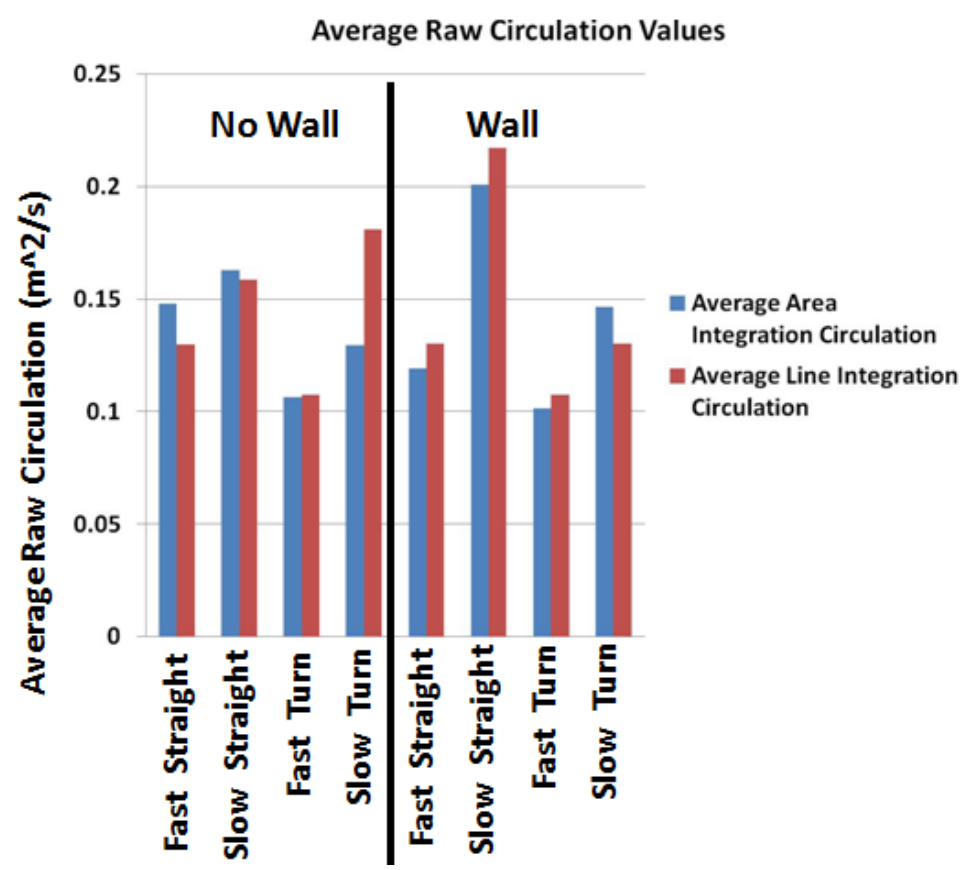

Figure 6.6: Similar average raw circulation values are seen for the area and line integration circulation calculations for wingtip vortices in this thesis research.

While the results in Figure 6.6 validate the circulation values that are calculated from the line and area integration approaches, both methods do not provide clear results that show a difference between the circulation of the vortex with or without the wall present. The wall effect does not seem to be apparent through the circulation calculations because the difference in circulation most likely falls within the uncertainty associated with the PIV method used. When PIV velocity data is used to calculate derivative quantities and then these derivative quantities are integrated, the uncertainty can be as large as $20 \%$. Since the Night Vapor is manually flown in this research, it is not possible to make the vortex appear in the same location for each test and thus it is not possible to 
average images and decrease this uncertainty. The calculations that contribute to the PIV uncertainty for this test are discussed in Appendix A.

While the circulation values of the wingtip vortex from the PIV testing do not provide a clear view of how the wall affects the wingtip vortex and the flight controls of the MAV, some trends are noticed in the circulation calculations that relate to the formation of a wingtip vortex in general. Elliptical instabilities located within the wingtip vortex may provide a more clear understanding of the wall effect on the vortex and the MAV flight controls.

\subsubsection{Wingtip Vortex Ellipticity}

Measuring the ellipticity of the wingtip vortices helps to investigate how the wall deforms the vortices and provides insight into how the vortex wall interactions affect the MAV flight controls. As discussed in section 3.5, the wingtip vortices near the wall could be considered to have moderate ellipticity and the wingtip vortices without the wall could be considered to have low ellipticity. The ellipticity is calculated by dividing the major vortex axis by the minor vortex axis. Therefore, the ellipticity ratios will have a value greater than one with the vertical axis having the larger value. Figure 6.7 shows the ellipticity values calculated for each of the eight tests conducted in this research. All of the wall tests have higher ellipticity values than the no wall tests. While a perfect circular vortex would have an ellipticity value of one, because the major and minor axis lengths would be the same, the no wall tests do have some ellipticity. The variance in the ellipticity is calculated through measuring the major axis of a wingtip vortex near a wall multiple times and determining the standard deviation of these measurements. The 
standard deviation is $0.29 \mathrm{~mm}$ which is approximately $0.23 \%$ of the median measured value of $125.00 \mathrm{~mm}$. This result is rather precise due in large part to the clearly defined vortex boundary yielded from the $\Gamma_{1}$ and $\Gamma_{2}$ method of Graftieaux [31]. Figure 6.7 helps to validate the thought that the wingtip vortices near the wall have moderate ellipticity while the no wall tests have low ellipticity.

\section{Vortex Ellipticity}

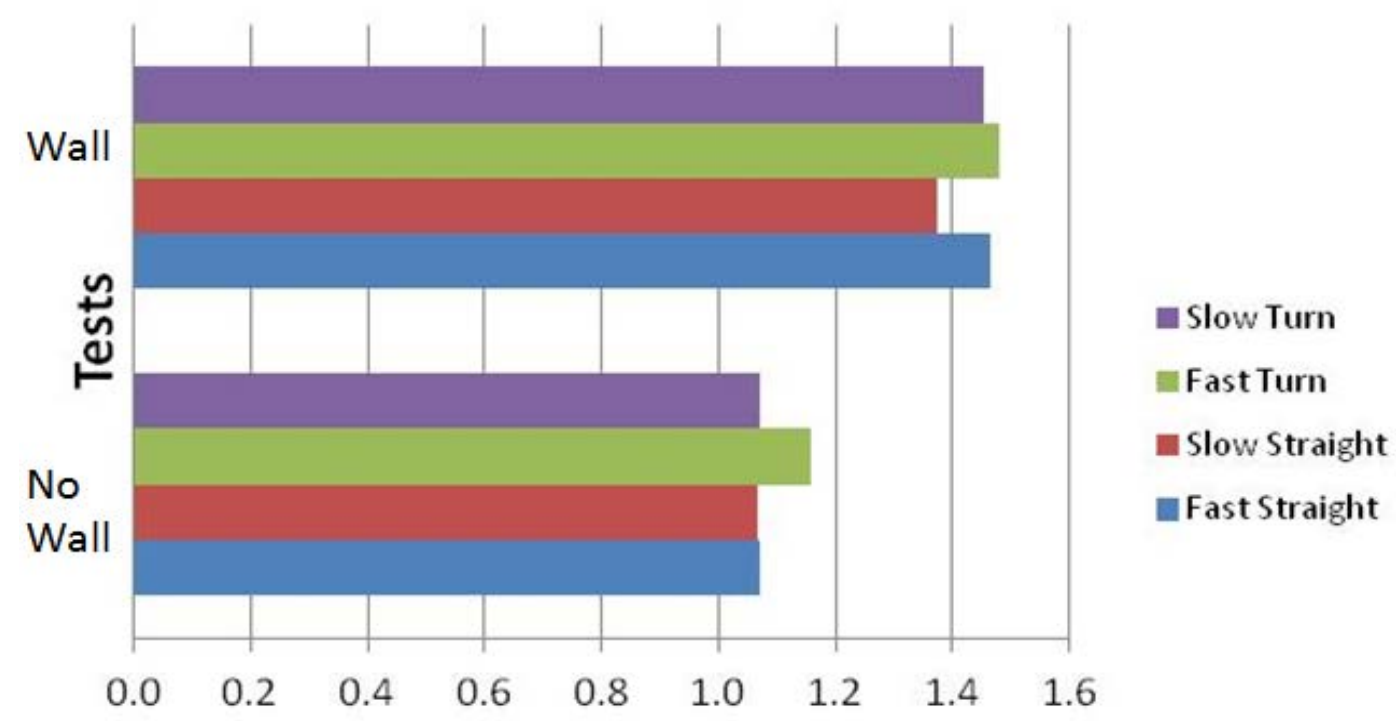

\section{Ellipticity}

Figure 6.7: This plot of the ellipticity values for each test condition shows that the wingtip vortices near a wall always have a higher ellipticity value.

Once the ellipticity values for the different test conditions are known, it is possible to investigate the instabilities associated with the different levels of ellipticity. As discussed in section 3.5, a vortex with moderate ellipticity should have a dominant three-dimensional instability located at the core as well as a weaker three-dimensional instability at the edge of the vortex boundary. In contrast, the wingtip vortex without the 
wall has low ellipticity and should have a dominant three-dimensional instability located at the boundary edge of the vortex and an additional weaker instability in the vortex core.

Looking at the normalized velocity contour plots of the tests with and without the wall will help to visualize these instabilities. Figure 6.8 shows a normalized velocity contour plot of a wingtip vortex from a slow turning test near the wall. The black square represents the boundary of the vortex defined by the $\Gamma_{2}$ criteria. The core of this vortex looks unstable due to the dominant instability present in the vortex core for a moderately elliptical vortex. A weaker instability can also be seen at the edge of the wingtip vortex between the wall and the vortex $\Gamma_{2}$ boundary. This weaker edge instability is also due to the fact that the wingtip vortex near the wall has moderate ellipticity. In contrast, Figure 6.9 shows a normalized velocity contour plot of a wingtip vortex from a slow straight flight without the wall present. The black box again represents the boundary of the vortex from the $\Gamma_{2}$ criteria. It is difficult to visualize the strong instability at the vortex boundary or the weaker instability at the vortex core that a vortex with low ellipticity should contain. This leads to the belief that the wingtip vortices without the wall present should actually be defined as having somewhere between zero to low ellipticity. This definition seems fitting since the ellipticity values for these tests are slightly greater than one but the instabilities are not readily seen in a normalized velocity contour plot. A vortex-vortex interaction could contribute to the small amount of ellipticity seen in the wingtip vortices without the wall present. This vortex-vortex interaction is a result of a fluid shear from the opposite wingtip vortex. This fluid shear from the opposite wingtip vortex is present for the tests conducted with or without the wall present. This vortexvortex interaction will be discussed further shortly. Some of the instabilities seen in the 
wingtip vortex near a wall in Figure 6.8 could be argued to also originate from natural instabilities created during the formation of the wingtip vortex. However, it is believed that most of the instabilities originate from the ellipticity of the vortex since there is a significantly larger amount of instability observed in the wingtip vortex near the wall compared to the wingtip vortex without the wall present.

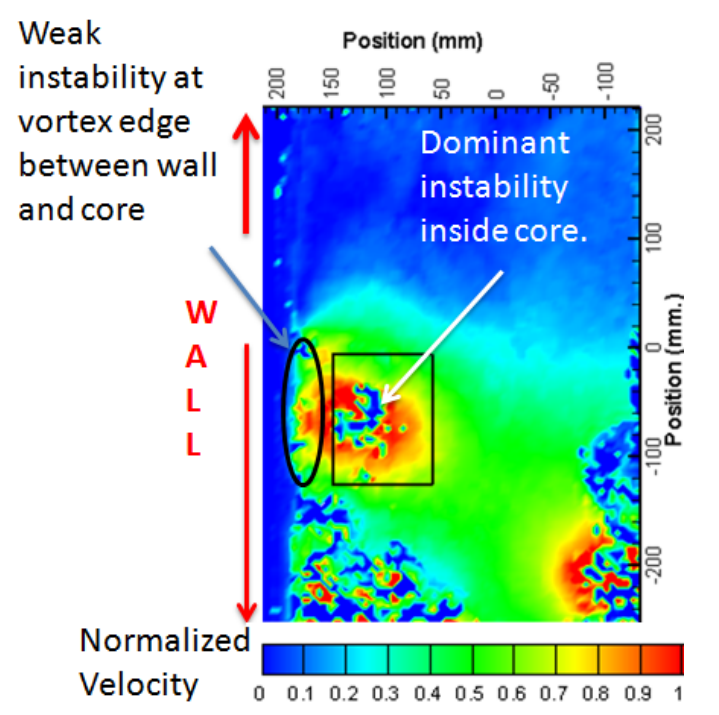

Figure 6.8: The dominant instability and the weaker edge instability are shown in this normalized velocity contour plot from a slow turning flight near the wall.

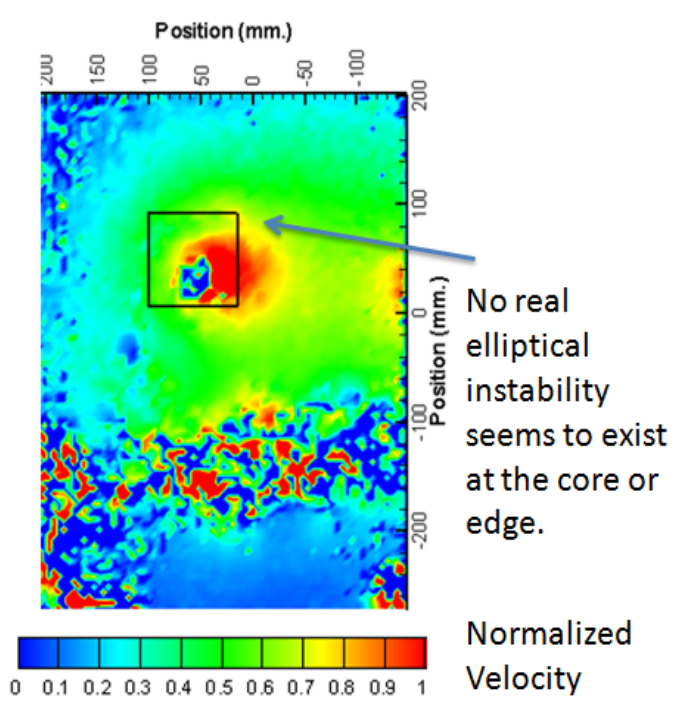

Figure 6.9: Normalized velocity contour plot for a wingtip vortex from a slow straight flight without the wall shows a lack of instabilities.

Since the elliptical instabilities seem to be prominent in the vortex core for the tests conducted near the wall, it is thought that these instabilities will cause inconsistent circulation values for the wall tests compared to the no wall tests. However, as previously discussed, little difference is noticed within the circulation calculations for the tests conducted with or without a wall present.

Looking at the axis alignment for which the elliptical wingtip vortices elongate for the tests near the wall also provides insight into how the wingtip vortex interacts with 
the wall and how a MAV's flight controls might be affected. Figure 6.10 shows a wingtip vortex from a fast turning test conducted near the wall. The yellow vertical line at the core of this vortex demonstrates that the vortex core elongates in the vertical direction. This can also be explained by the fact that the wingtip vortex experiences a solid surface friction shear from the wall while also simultaneously experiencing the fluid shear from the opposite wingtip vortex as a result of the vortex-vortex interaction discussed earlier. This dual shear force on the wingtip vortex near a wall contributes to its elongation in the vertical direction. In contrast, Figure 6.11 shows the circular nature of a wingtip vortex from a fast turning test without the wall present. Overall, this dual shear contributes to the elliptical instabilities seen in the wingtip vortices near the wall and this would affect a MAV's flight controls while flying near a wall.

The identification of these elliptical instabilities and dual shear instabilities support the main goal of this research in determining how the MAV's flight control is affected as a result of the wingtip vortex interacting with a wall. A MAV's flight controls will be affected by the strong instabilities present within the vortex core and the secondary instabilities located between the vortex and the wall for the tests conducted with a wall present.

Finally, Figure 6.10 also reveals a secondary vortex which has the opposite sense rotation compared to the primary wingtip vortex. This secondary vortex will be discussed next. 


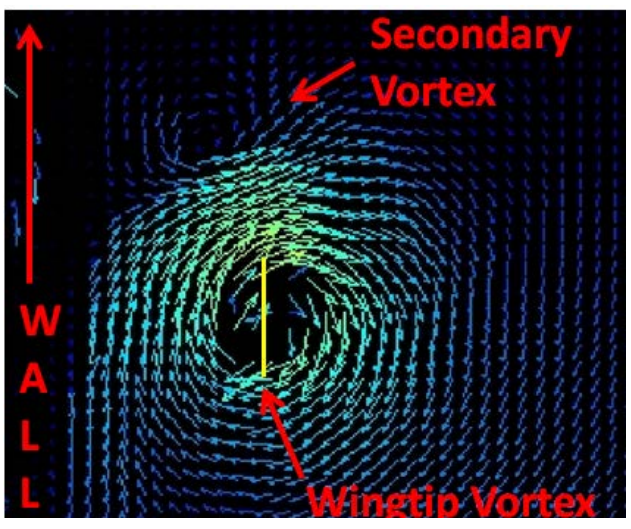

Figure 6.10: The yellow line shows the vertical major axis of the elliptical shaped wingtip vortex near a wall. Secondary vortex also identified.

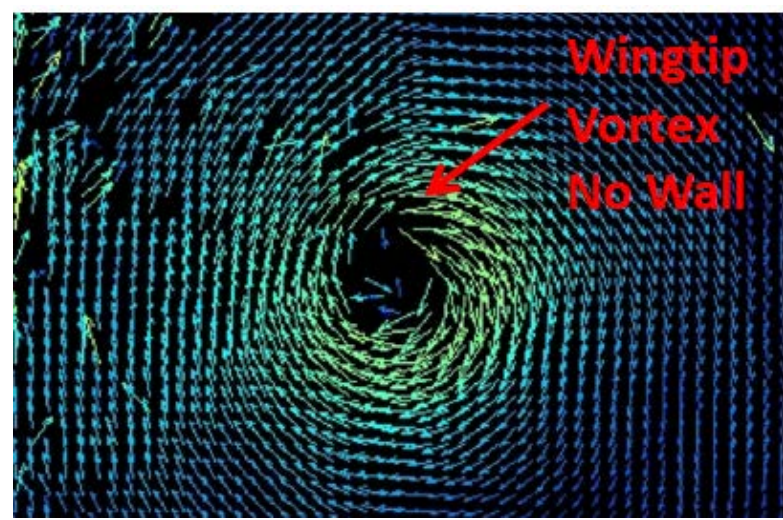

Figure 6.11: The velocity vectors in this image form a more circular shape compared to that of Figure 6.10 since a wall is not present in this test.

\subsubsection{Secondary Vortex due to Wall Effect}

The secondary vortex pointed out in Figure 6.10 is a direct result of the aerodynamic interactions between the wingtip vortex and the wall. In order to understand this secondary vortex from the wall effect and its effect on the controls of a MAV, it is necessary to look at the temporal evolution of the wingtip vortex interacting with the wall. The top row in Figure 6.12 shows seven consecutive velocity vector field plots of the vortex tube for a fast turning test near the wall. The time values located below each image refer to the time after the Night Vapor has left the field of view. The horizontal white line represents the elevation of the center of the wingtip vortex core from the first image at 0.2 seconds. As time passes the wingtip vortex rises in elevation and moves away from the wall. By the last image at 0.8 seconds, the wingtip vortex core center has dropped back to the elevation of the white line and is farther away from the wall compared to the first image. After the last image, the vortex continuously drops in 
elevation and leaves the field of view. This slight rise in vortex elevation and movement away from the wall indicate a vortex rebound off the wall. However, the movement of the vortex tube described in this paragraph may not entirely be due to the wall effect alone. As described by Igarashi et al [27] in section 2.8, wingtip vortex wandering is a common phenomenon. At this point in time it is difficult to separate how much of the movement of the wingtip vortex seen in this research is caused by the vortex tube rebounding off of the wall and how much is caused by the natural phenomenon of wingtip vortex wandering. However, it is certain that the wingtip vortex tube seen in Figure 6.12 experiences an upward movement away from the wall and that this will have an effect on a MAV's flight controls when flying near a wall.

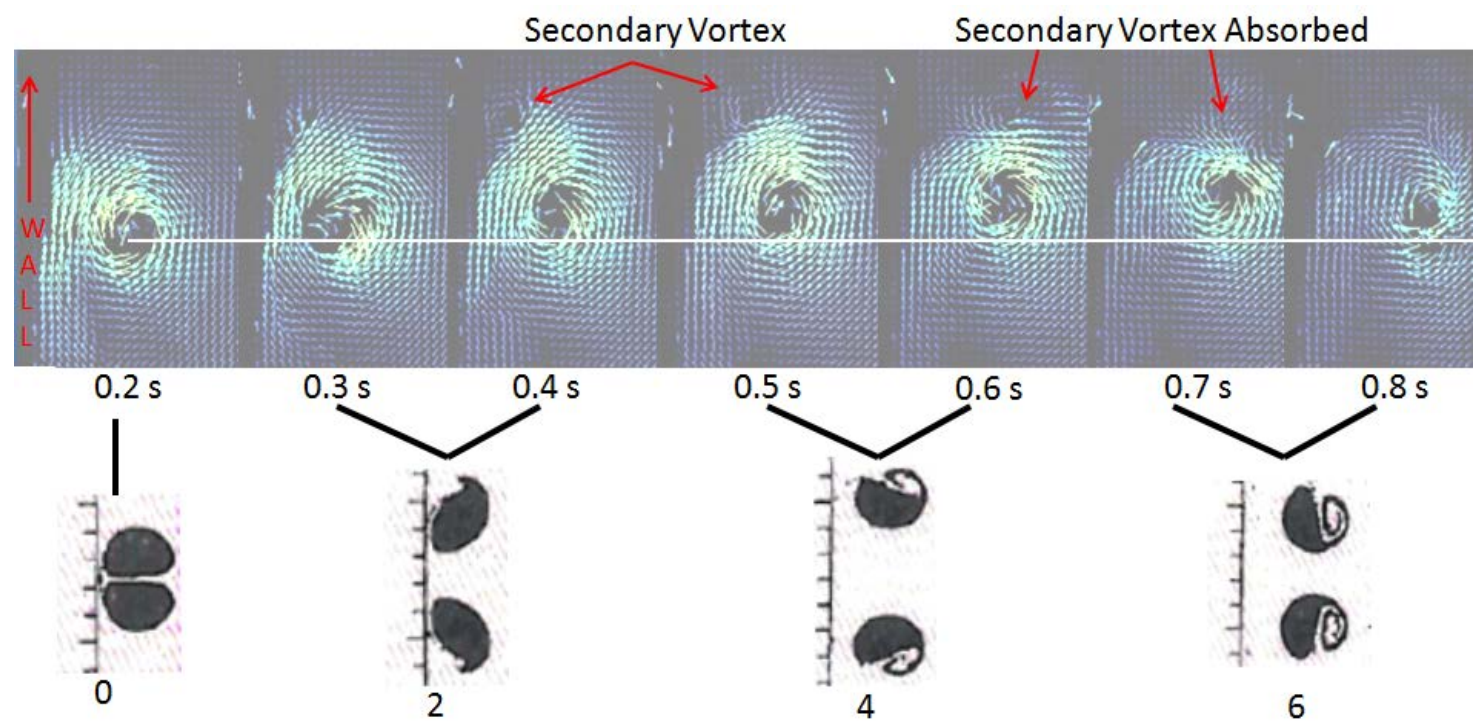

Figure 6.12: Top Row: Time evolution of the wingtip vortex as seen in the velocity vector field plots. The vortex core moves up and away from the wall as indicated by its position above the white line. Bottom Row: Nondimensional time evolution of vortex dipole particles impacting a wall from a particle-in-cell method by Ho Liu and Doorly [23]. This shows a similar secondary vortex develop.

The secondary vortex is not visible at the $0.2 \mathrm{~s}$ time frame, develops in the 0.3 to $0.4 \mathrm{~s}$ time frames, follows a circular pattern around the primary vortex in the 0.5 to $0.6 \mathrm{~s}$ time frames, and is absorbed back into the primary vortex in the 0.7 to $0.8 \mathrm{~s}$ time frames. 
In order to explain the role that the secondary wingtip vortex plays in the primary vortex's rebound off of the wall, a paper by Ho Liu and Doorly [23] is reexamined. This paper also helps to investigate how this phenomenon contributes to the aerodynamic interaction between the wall and the MAV's wingtip vortex and ultimately how this affect's the MAV's flight controls.

The bottom row of images in Figure 6.12 is from the paper by Ho Liu and Doorly [23]. These images represent the nondimensional temporal evolution of vortex dipole particles impacting a wall at a perpendicular angle and are derived through using a particle-in-cell (PIC) method. This research actually looks at a vortex descending and impacting a horizontal wall, but the wall and vortex images seen here have been rotated $90^{\circ}$ so that the wall is vertical. This makes it easier to compare the results from this literature to the wingtip vortex wall effects seen in this thesis research. The general idea of the vortex evolution discussed here will remain the same if the vortex dipole is rotated $90^{\circ}$. Looking only at the top half of the vortex dipole at a nondimensional time value of 0 , it is possible to see that the top vortex is near the wall, just like the $0.2 \mathrm{~s}$ time frame image from this thesis research. At a nondimensional time value of 2 , the top half of the vortex dipole impacts the wall and a secondary vortex of opposite sign is created above the primary vortex, just like time frames 0.3 to $0.4 \mathrm{~s}$ in this thesis research. During the nondimensional time value 4 , the secondary vortex from the top half of the vortex dipole gains strength, moves upward, and restricts the vertical motion of the primary vortex. The primary vortex rebounds from the wall and the secondary vortex moves along a circular trajectory around the primary vortex. This motion of the secondary vortex is also seen in the 0.5 to $0.6 \mathrm{~s}$ time frame of this thesis research. Finally, during the 
nondimensional time value of 6 , the top half of the vortex dipole's secondary vortex is swept around the primary vortex and is eventually absorbed back into the primary vortex [23]. In the 0.7 to $0.8 \mathrm{~s}$ time frames for this thesis research, the secondary vortex is completely absorbed back into the primary wingtip vortex. This shows that the development and motion of the secondary vortex due to the wall effect seen in this thesis research is consistent with the results seen in the literature for a vortex impacting a wall. The theory behind how this secondary vortex develops and its impact on a MAV's flight controls are discussed next.

Ho Liu and Doorly's [23] theory helps to explain how the secondary vortex forms for this thesis research. Ho Liu and Doorly [23] explain that in their research, they do not include a preexisting wall shear layer at the wall surface, which is the same approach taken in this thesis research. Instead it is seen that when the vortex core impacts the wall, an induced boundary layer is created along the wall. This boundary layer indicates that a velocity gradient and viscous shear stress exist due to the presence of the wall and the friction between the flow and the wall. If the vortex is extremely close to the wall, this induced boundary layer along the wall separates from the wall when it experiences an adverse pressure gradient as the flow slows down well after it passes between the tight gap between the vortex core and the wall. This adverse pressure gradient is created since the flow moves from a high to low velocity region (low to high pressure region). The separation bubble caused by this adverse pressure gradient grows quickly and expels the flow away from the wall and forms the secondary vortex [23]. See Figure 6.13 to visualize the separating velocity vectors as they experience the adverse pressure gradient after passing between the vortex core and wall. 


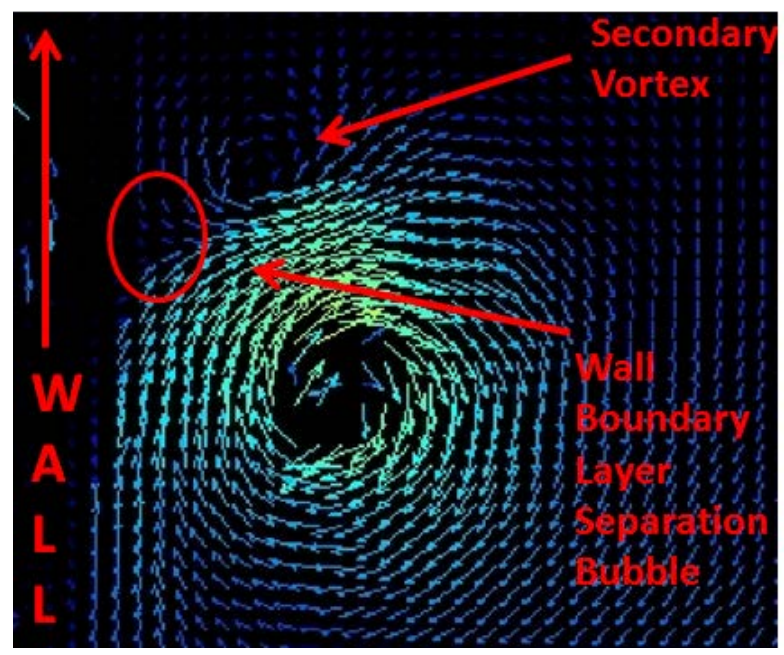

Figure 6.13: Formation of the secondary vortex due to separation.

The separation bubble that forms the secondary vortex causes the primary wingtip vortex to rebound and move up away from the wall as seen in Figure 6.12. From a global perspective, this means that the entire vortex tube along the aircraft is rebounding from the wall. This is a significant interaction between the wingtip vortex and the wall because the wall plays a role in this phenomenon. This creation and dissipation of the secondary wingtip vortex and rebound of the primary wingtip vortex only takes 0.8 seconds to occur and thus the aircraft is still in close proximity to this interaction. Thus it has an effect on the global view of the aerodynamic interactions between the MAV's wingtip vortex and the wall, which will have an effect on the MAV's flight controls. While all of the results thus far are derived from the free flight PIV testing, the wind tunnel testing performed in this thesis also investigates how the MAV's flight controls are affected as a result of the wingtip vortex interacting with a wall. 


\subsection{Wind Tunnel Results}

The wind tunnel testing is important in this thesis research because it provides a more controlled environment in which to investigate the aerodynamic interactions between a MAV and a building wall compared to the free flight PIV tests. This will help to achieve the overall goal of determining how a MAV's flight controls are affected as a result of flying near a wall. The X-EC airplane provides the most conclusive wind tunnel results since the sting force balance is not sensitive enough for the delicate Night Vapor aircraft. Overall, an increase in the aircraft's $C_{L}$ is noticed as the wall is positioned closer and closer to the wingtip vortex for both the straight and turning wind tunnel tests at fast and slow velocities.

Figure 6.14 shows a plot for the aircraft $\mathrm{C}_{\mathrm{L}}$ vs. the distance between the wingtip and the wall for the fast and slow straight tests performed in the wind tunnel with and without a wall present. The data points with an $\mathrm{x}$ value of about $20 \mathrm{~cm}$ are the tests performed without the wall present. This distance is the measured distance between the wingtip and the side wall of the wind tunnel test section. The fast straight data points show a $6.02 \%$ increase in $\mathrm{C}_{\mathrm{L}}$ between the no wall test and the one $\mathrm{cm}$ away from the wall test. The slow straight data points show a $7.46 \%$ increase in $\mathrm{C}_{\mathrm{L}}$ between the no wall test and the one cm away from the wall test.

Figure 6.15 shows a similar plot for the fast and slow turning tests performed in the wind tunnel with and without a wall present. These tests are pure bank tests with no rudder deflection as would have been seen in the free flight tests. It is expected that this difference will have little bearing on the overall $C_{L}$ of the aircraft. The fast turning data points show an $8.79 \%$ increase in $\mathrm{C}_{\mathrm{L}}$ between the no wall test and the one $\mathrm{cm}$ away from 
the wall test. The slow turning data points show a $5.97 \%$ increase in $C_{L}$ between the no wall test and the one cm away from the wall test.

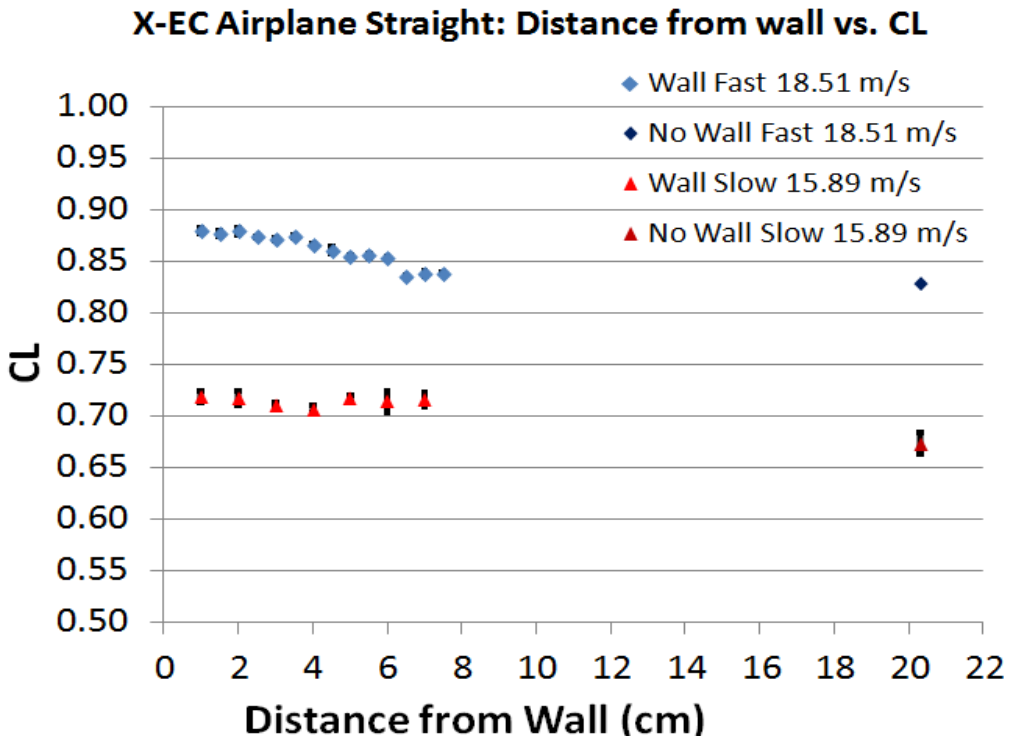

Figure 6.14: Fast straight and slow straight wind tunnel results show an increase in $\mathrm{C}_{\mathrm{L}}$ as the wall approaches the wingtip.

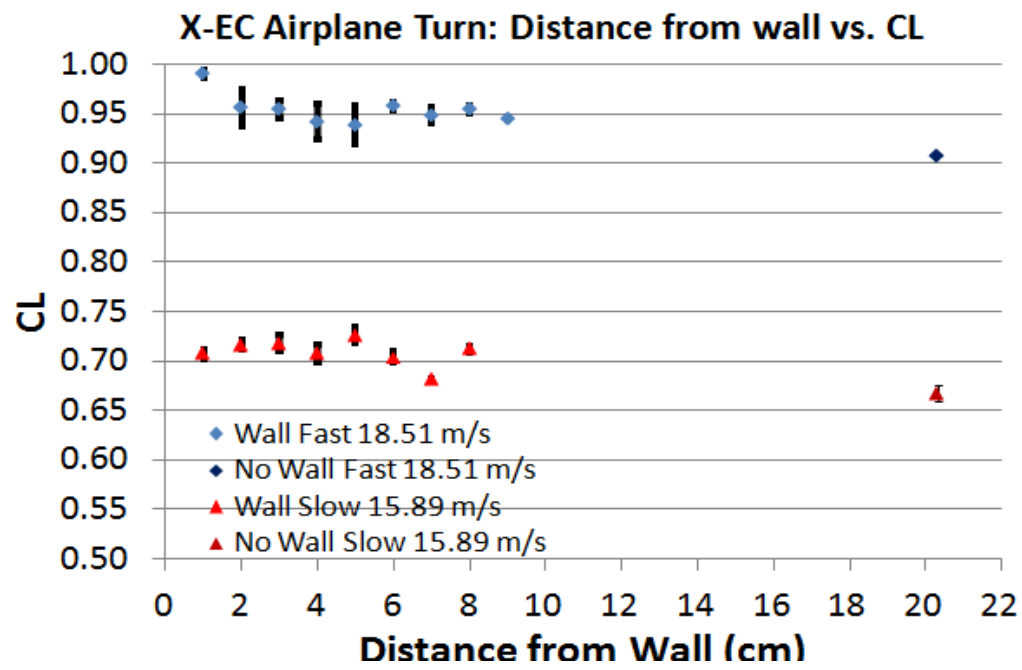

Figure 6.15: Fast turning and slow turning wind tunnel results show an increase in $\mathrm{C}_{\mathrm{L}}$ as the wingtip nears the wall. 
It is also possible to compare the flight maneuvers to the $\mathrm{C}_{\mathrm{L}}$ values seen in the wind tunnel tests. Table 6.2 shows that there is a $12.5 \%$ increase in $\mathrm{C}_{\mathrm{L}}$ between the fast straight and the fast turning tests one $\mathrm{cm}$ away from the wall. Table 6.2 also shows that there is a $9.64 \%$ increase in $C_{L}$ between the fast straight and the fast turning tests without the wall present. Table 6.2 also shows that $22.88 \%$ of the total increase in lift at the one cm away from the wall test case is a result of the wall while the other $77.12 \%$ of the total increase in $\mathrm{C}_{\mathrm{L}}$ at one $\mathrm{cm}$ away from the wall is due to the fact that the airplane is flying in a banked turn. Therefore, it can be seen that the turn contributes to most of the increase in $\mathrm{C}_{\mathrm{L}}$ at one cm away from the wall, but the wall still contributes a significant amount to this increase in lift.

Table 6.2: When comparing the percentage of increase in $\mathrm{C}_{\mathrm{L}}$ for different maneuvers a higher percentage of increase in $C_{L}$ exists near the wall.

\begin{tabular}{|c|c|c|c|c|}
\hline Maneuver & $\begin{array}{c}\text { Distance Between } \\
\text { Wall and Wingtip at } \\
\text { Max } C_{\mathrm{L}}(\mathrm{cm})\end{array}$ & Max $\mathrm{C}_{\mathrm{L}}$ Value & $\begin{array}{c}\text { Increase in } \\
\mathrm{C}_{\mathrm{L}}(\%)\end{array}$ & $\begin{array}{c}\text { Percentage of } 12.5 \% \\
\text { Increase in } \mathrm{C}_{\mathrm{L}} \text { When } \\
\text { Wall is One cm Away }\end{array}$ \\
\cline { 1 - 3 } Fast Straight & 1 & 0.88 & \multirow{2}{*}{12.5} & $22.88 \%$ due to wall \\
${$\cline { 1 - 3 }$} }$ & 1 & 0.99 & & $77.12 \%$ due to turn \\
\cline { 1 - 3 } Fast Straight & No Wall & 0.83 & \multirow{2}{*}{9.64} & \\
\hline Fast Turning & No Wall & 0.91 & & \\
\hline
\end{tabular}

Overall, Table 6.2 shows that the fast turning maneuver always has a higher $\mathrm{C}_{\mathrm{L}}$ than the fast straight maneuver regardless of the wall's distance from the wingtip. However, it can also be seen that the flight maneuver also has a combined effect with the wall effect since the turning maneuver near the wall has a higher $\mathrm{C}_{\mathrm{L}}$ compared to the turning maneuver without the wall. This combined maneuver and wall effect increase in $C_{L}$ has an effect on the MAV flight controls for the fast flights. 
The slow fights do show an increase in $\mathrm{C}_{\mathrm{L}}$ between the tests conducted without the wall present compared to the tests conducted one $\mathrm{cm}$ away from the wall. However, there is no clear difference in $\mathrm{C}_{\mathrm{L}}$ when comparing the turning and the straight tests. The increase in $\mathrm{C}_{\mathrm{L}}$ is also inconsistent as the wall gradually moves closer to the wingtip.

Both Figures 6.14 and 6.15 contain error bars at each data point. These error bars are based on the standard deviations of the test measurements at each condition.

Overall, the wind tunnel testing shows that the wall effect increases the $\mathrm{C}_{\mathrm{L}}$ on the aircraft, especially for a fast banked turning maneuver. In order to put these changes in $\mathrm{C}_{\mathrm{L}}$ into context, the velocity triangle equivalent vertical gust is calculated for each test condition. This helps to calculate the load factor on the airplane and the vertical velocity of the gust at the wall. Table 6.3 shows the results of these calculations. The fast tests always have the higher load factor and the higher vertical velocity compared to the slow tests with the same maneuver. The fast turning test has the highest overall load factor and vertical gust velocity due to the banked turn. Overall, Table 6.3 shows that when flying at speeds of 15.89 and $18.51 \mathrm{~m} / \mathrm{s}$, a MAV can expect to have a load factor between 1.07 to 1.16 and a vertical gust velocity of 0.15 to $0.34 \mathrm{~m} / \mathrm{s}$ while flying one $\mathrm{cm}$ away from a wall. A MAV will have to adjust its flight controls in order to account for a change in $\mathrm{C}_{\mathrm{L}}$ when flying near the wall. While wind tunnel testing is one form of research that helps investigate this topic in a more controlled manner compared to the free flight PIV experiments, a computer simulation is another method of testing in a more controlled environment. 
Table 6.3: Highest wing loading and vertical gust velocities are seen for the fast turning test one $\mathrm{cm}$ away from the wall.

\begin{tabular}{|c|c|c|c|c|}
\hline $\begin{array}{c}\text { Wind Tunnel Testing Load Factor and } \\
\text { Vertical Velocity One cm from Wall }\end{array}$ & Slow Straight & Fast Straight & Slow Turn & Fast Turn \\
\hline Load Factor on X-EC Airplane & 1.07 & 1.10 & 1.08 & 1.16 \\
\hline $\begin{array}{c}\text { Vertical Velocity of the Gust Due to } \\
\text { Wall (m/s) }\end{array}$ & 0.18 & 0.21 & 0.15 & 0.34 \\
\hline $\begin{array}{c}\text { Velocity of Gust as Percentage of } \\
\text { Tunnel Velocity (\%) }\end{array}$ & 1.13 & 1.13 & 0.94 & 1.84 \\
\hline
\end{tabular}




\section{CHAPTER 7}

\section{CONCLUSIONS}

The theory behind this research involves the fact that a vortex has an influence on a body or flow in its environment as a function of the distance between the vortex and the body or flow. The aerodynamic interactions between a MAV's wingtip vortex and a wall are investigated in order to determine if there is a wall effect on the MAV's flight controls.

Through performing free flight PIV testing on an RC aircraft flying near a wall, it is concluded that the wall effect creates a moderately elliptical wingtip vortex with threedimensional elliptical instabilities. The strongest instabilities are located within the core of the moderately elliptical wingtip vortex near the wall. The wall partially blocks the edge of the wingtip vortex and causes the weak edge instability to exist between the wall and the core. These instabilities can be visualized by looking at the core and edge of the wingtip vortex in a normalized velocity contour plot. The instability between the vortex edge and the wall decreases the wing downwash and increases the aircraft coefficient of lift in a similar fashion compared to ground effect, except that this effect is only applied to one wingtip. This decrease in wing downwash and increase in aircraft coefficient of lift due to the wall effect leads to an increase in effective angle of attack of the wingtip near the wall. The wall affects the control of the MAV since the effective angle of attack 
of the wingtip near the wall increases. Since only one wingtip experiences an increase in effective angle of attack, this means that the aircraft will experience a rolling motion away from the wall. A MAV must adjust its control surfaces in order to correct its flight path and counteract this rolling motion when flying near the wall.

The PIV free flight testing also reveals a secondary vortex which forms as a result of the aerodynamic interaction between the wingtip vortex and the wall. The vortex tends to rebound off of the wall and move up slightly and away from the wall due to the induced boundary layer along the wall separating and forming this secondary vortex. The phenomenon of vortex wandering also explains some of this vortex movement. The entire vortex tube rebounds off of the wall and the upward movement of this vortex tube causes the MAV wingtip near the wall to experience a higher coefficient of lift. This higher coefficient of lift is only applied to the wingtip near the wall and this would again indicate that the MAV would experience a rolling motion away from the wall. When flying near a wall, it would be necessary for a MAV to correct its flight controls to account for the wingtip vortex rebound off of the wall.

The wind tunnel testing also reveals valuable information about how a MAV's flight controls are affected as a result of flying along a wall. The results show an increase in the aircraft's coefficient of lift when the wingtip is located near the wall. The higher velocity at the wingtip due to a small wingtip wall gap and a lower pressure on top of the wing causes an increase in lift at the wingtip near the wall. This higher lift at the wingtip near a wall causes roll instability in the MAV. The aircraft's flight controls are affected because the aircraft wants to roll away from the wall due to the higher coefficient of lift experienced at the wingtip near the wall. The MAVs control surfaces must be adjusted in 
order to correctly compensate for the aircraft roll due to flying near a wall. For a fast banked turn near the wall, the wall effect is stronger, the coefficient of lift is higher, and the aircraft will roll more compared to a fast straight flight near the wall. The fast banked turning maneuver increases the MAV's coefficient of lift to begin with but the wall does contribute to a significant percentage of the total increase in the coefficient of lift in a fast banked turning maneuver near the wall. This means that a MAV must correct its flight controls more significantly for a fast turning maneuver near the wall. MAVs will have to adjust their flight controls as a result of flying near a wall for mission success, especially during fast turning flights near a wall. MAVs must be capable of performing turning maneuvers near walls on a regular basis while flying in the urban environment and the ability to adjust the flight controls accordingly is imperative for mission success. 


\section{CHAPTER 8}

\section{RECOMMENDATIONS}

The PIV free flight wall effects testing can be expanded upon in several ways. One of the original goals of this research involves using an automated flight controller to fly the Night Vapor along the wall during the PIV testing. This would make it possible to average PIV images of the wingtip vortex in the same location for multiple flights. The ability to average multiple PIV images would help to minimize the errors in circulation calculations due to inconsistencies located at the edge of the vortices. The flight controller still needs some modifications in order to make it accurate enough to average PIV images but the spatial accuracy required in order to correctly average PIV images for an off the shelf RC aircraft in free flight are also exceptionally strict. Many other variables outside of the controller's accuracy also make free flight PIV averaging difficult, such as the battery drain on the aircraft as well as the limits of the off the shelf aircraft's hardware.

The PIV testing could also be expanded upon through performing additional experiments to separate the effect of wingtip vortex wandering from the wingtip vortex

rebound off of the wall. The ability to separate these effects could help to clearly identify how the wingtip vortex wall effect changes the MAV's flight controls. 
Several different MAV vehicle designs could also be used in the PIV free flight testing to determine how much the wingtip vortex wall interaction depends on the design of the MAV itself. Additional MAV fixed wing vehicles as well as flapping wing and rotorcraft MAVs could be used in the free flight PIV experiments to determine how these drastically different MAV designs are affected by wall effects.

While great advances are made in the PIV seeding between the proof of concept and final testing, a coanda nozzle system of seeding the air inside the small room in the flight lab might also help to create a more uniform seeding.

Several areas of the wind tunnel testing can also be expanded upon. It might be worthwhile to perform PIV in a wind tunnel in order to have the capability of averaging PIV images of the wingtip near a wall. The results from this research may provide insight into how successful the free flight PIV images may be with a more consistent fixed wing controller. If averaged PIV images from a wind tunnel test still do not yield new and clear results, a perfect fixed wing controller will also most likely struggle with similar problems. A more sensitive force balance could also be implemented in the UD wind tunnel in order to perform the wind tunnel testing with the delicate Night Vapor aircraft. This would make it possible to accurately test the free flight PIV test conditions in the UD wind tunnel.

Additional wind tunnel testing could also investigate a multitude of bank angles. Since the highest $\mathrm{C}_{\mathrm{L}}$ for this research is seen in the fast banked turning maneuver testing, it may be worthwhile to test at a variety of different bank angles to determine if there is an angle that provides the most increase in $\mathrm{C}_{\mathrm{L}}$ and thus the most roll. 
Wind tunnel testing could be expanded to test how the wingtip vortex interacts with different wall surfaces and how this affects the flight controls of the MAV. Brick, drywall, wooden paneling, and cinder blocks are all examples of different building materials that often form the inside or outside of buildings and walls. These drastically different materials all have different surface textures and could cause drastically different wingtip vortex wall interactions. Therefore, these diverse surfaces could drastically affect the MAV flight controls differently.

Finally, additional wind tunnel testing could be conducted to test how the wingtip vortices interact with a wall as the vehicle flies directly over a thin wall structure, such as the solid backrest of a chair for example. As the wingtip vortices descend, the thin wall will separate both of the wingtip vortices from interacting with each other and prevent a vortex-vortex shear force. This wall effect could also cause an effect on the MAV flight controls.

Numerical methods of modeling the wingtip vortex wall interaction and the effects on the control of a MAV can also be investigated. A vortex lattice method may provide a successful platform for creating a computer model to simulate the wingtip vortex interaction with a wall. 


\section{REFERENCES}

1. Abate, G., O1, M., Shyy, W., "Introduction: Biologically Inspired Aerodynamics," AIAA Journal, Vol. 46, No. 9, 2008.

2. Orr, M. W., Rasmussen, S. J., Karni, E. D., \& Black, W. B., "Framework for Developing and Evaluating MAV Control Algorithms in a Realistic Urban Setting," AIAA Paper, 2005.

3. Abdulrahim, M., Watkins, S., and Segal, R., "Dynamic Sensitivity to Atmospheric Turbulence of a Fixed-Wing MAV with Varying Configuration," AIAA Paper, 2009.

4. Watkins, S., Loxton, B. J., and Milbank, J., "Wind-Tunnel Replication of Atmospheric Turbulence with an Emphasis on MAVs," AIAA Paper, 2008.

5. Watkins, S., Milbank, J., and Loxton, B. J., "Atmospheric Winds and Their Implications for Micro Air Vehicles,” AIAA Journal, Vol. 44, No. 11, 2006, pp. 2591-2600.

6. Shim, D. H., Chung, H., Jin Kim, H., and Sastry, S., "Autonomous Exploration in Unknown Urban Environments for Unmanned Aerial Vehicles," AIAA Paper, 2005.

7. Kurdila, A., Nechyba, M., Prazenica, R., Dahmen, W., Binev, P., DeVore, R., and Sharpley, R., "Vision-Based Control of Micro-Air Vehicles; Progress and Problems in Estimation," IEE Paper, 2004.

8. Prazenica, R. J, Kurdila, A. J., Sharpley, R. C., and Evers, J., "Multiresolution and Adaptive Path Planning for Maneuver of Micro-Air-Vehicles in Urban Environments," AIAA Paper, 2005.

9. Hall, J. K., and McLain, W., "Aerobatic Maneuvering of Miniature Air Vehicles Using Attitude Trajectories," AIAA Paper 2008.

10. Ginevsky, A., and Zhelannikov, A., "Vortex Wakes of Aircrafts," Springer-Verlag, Berlin Heidelberg, 2009, Chaps. 1. 
11. Hong, Y., and Altman, A., "Vortex Lift Contributions in the Spanwise Plane in Flapping Wings," $24^{\text {th }}$ Applied Aerodynamics conference, AIAA paper 2006.

12. Chow, J. S., Zilliac, G. G., and Bradshaw, P., "Mean and Turbulence Measurements in the Near Field of a Wingtip Vortex," AIAA Journal, Vol. 35, No. 10, October 1997, pp. 1561- 1567.

13. Lian, Y., and Shyy, W., "Numerical Simulations of Membrane Wing Aerodynamics for Micro Air Vehicle Applications," Journal of Aircraft, Vol. 42, No. 4, 2005, pp. 865-873.

14. Anderson, J. D., Jr., "Introduction to Flight," $3^{\text {rd }}$ ed. McGraw-Hill, New York, 1989.

15. Barnes, J. P., "The Elliptical Wing in-or-out of Ground Effect," Pelican Aero Group, Sailplane Builder, July-Aug-September 2009, 6 Nov. 2011

$<$ http://www.howfliesthealbatross.com/downloadables/Elliptical\%20Wing\%20in\%20 or $\% 20$ out $\% 20$ of $\% 20$ Ground $\% 20$ Effect.pdf $>$.

16. Boschetti, P., Cárdenas, E., Amerio, A., and Arévalo, A., "Stability and Performance of a Light Unmanned Airplane in Ground Effect," AIAA Paper, 2010.

17. Delisi, D., and Greene, G., "Experimental Measurements of the Evolution of a Vortex Pair in a Nonstratified Fluid Part 1: Migration and Persistence," AIAA Paper, 2009.

18. Mueller, T., J., "Aerodynamic Measurements at Low Reynolds Numbers for Fixed Wing Micro-Air Vehicles," RTO AVT/VKI Special Course on Development and Operations of UAVs for Military and Civil Applications, 1999.

19. Kaplan, S. M., Altman, A., and O1, M., "Wake Vorticity Measurements for Low Aspect Ratio Wings at Low Reynolds Number," Journal of Aircraft, Vol. 44, No. 1, 2007, pp. 241-251.

20. Zhual, L., and Gharib, M., "Near Field Dynamics of Wing Tip Vortices," $31^{\text {st }}$ AIAA Fluid Dynamics Conference and Exhibit, 2001.

21. Delisi, D., "Turbulence Measurements in the Wakes of aircraft and Model Laboratory Wings," $50^{\text {th }}$ AIAA Aerospace Sciences Meeting Including the New Horizons Forum and Aerospace Exposition, 2012.

22. Koumoutsakos, Petros, "Active Control of Vortex-Wall Interactions," Physics of Fluids, American Institute of Physics 1997, Vol. 9, No. 12, 1997, pp. 3808-3816.

23. Ho Liu, C., Doorly, D. J., "Velocity-Vorticity Formulation with Vortex Particle-inCell Method for Incompressible Viscous Flow Simulation, Part II: Application to Vortex/Wall Interactions," Numerical Hat Transfer, Part B, Vol. 35, 1999, pp. 277 294. 
24. Le Dizes, S., and Laporte, F., "Theoretical Predictions for the Elliptical Instability in a Two-Vortex Flow," Journal of Fluid Mechanics, Vol. 471, pp. 169-201, 2002.

25. Potylitsin, P. G., and Peltier, W. R., "Three-Dimensional Destabilization of Stuart vortices: the Influence of Rotation and Ellipticity," Journal of Fluid Mechanics, Vol 387, pp. 205-226. 1999.

26. Orlandi, P., "Vortex Dipole Rebound from a Wall," Physics of Fluids, Volume (2) No. 8, 1990, pp. 1429-1436.

27. Igarashi, H., Durbin, P. A., and Hu, H., "The Effects of Wind Tunnel Walls on the Near-field Behavior of a Wingtip Vortex," AIAA paper 2011.

28. Torres, G. E., Mueller, T. J., "Low-Aspect-Ratio Wing Aerodynamics at Low Reynolds Numbers," AIAA Journal Vol. 42, No. 5, 2004, pp. 865-873.

29. Spedding, G. R., "The Wake of a Kestrel (Falco Tinnunculus) in Gliding Flight," Journal of Experimental Biology, Vol. 127, pp. 45-57, 1987.

30. Robinson, S. K., "Coherent Motions in the Turbulent Boundary Layer," Annual Review Fluid Mechanics, 23:601-639, 1991.

31. Graftieaux, L., Michard, M., and Grosjean, N., "Combining PIV, POD and Vortex Identification Algorithms for the Study of Unsteady Turbulent Swirling Flows," Measurement Science and Technology, 2001, pp. 1422-1429.

32. Morgan, C. E., Babinsky, H., and Harvey, J. K., "Vortex Detection Methods for use with PIV and CFD data," $47^{\text {th }}$ AIAA Aerospace Sciences Meeting Including the New Horizons Forum and Aerospace Exposition, 2009.

33. Baik, Y. S., Bernal, L. P., and Shyy, W., "Unsteady Force Generation and Vortex Dynamics of Pitching and Plunging Flat Plates at Low Reynolds Number," $49^{\text {th }}$ AIAA Aerospace Sciences Meeting including the New Horizons Forum and Aerospace Exposition, 2011.

34. Katz, J., and Plotkin, A., "Low-Speed Aerodynamics," $2^{\text {nd }}$ edition, Cambridge University Press, Cambridge, 2001.

35. Mueller, T. J., and Delaurier, J. D., "Aerodynamics of Small Vehicles," Annual Review of fluid Mechanics, Vol. 35, 2003, pp. 89-111.

36. Noca, F., Shiels, D., and Jeon, D., “A Comparison of Methods for Evaluating TimeDependent Fluid Dynamic Forces on Bodies, Using Only Velocity Fields and their Time Derivatives," Journal of Fluids and Structures, Vol. 13, No. 1999.0219, 1999, pp. 551-578. 
37. Hong, Y., and Altman, A., "Streamwise Vorticity in Simple Mechanical Flapping Wings," Journal of Aircraft, Vol. 44, No. 5, 2007, p. 1592.

38. Lin, J. C., and Rockwell, D., "Force Identification by Vorticity Fields: Techniques Based on Flow Imaging," Journal of Fluids and Structures, Vol. 10, No. 6, Aug. 1996, pp. 663-668.

39. Lighthill, J., "Fundamentals Concerning Wave Loading on Offshore Structures," Journal of Fluid Mechanics, Vol. 173, 1986, pp. 667-681.

40. "Dantec Dynamics: Particle Image Velocimetry Measurement Principles," 3 March $2011<$ http://www.dantecdynamics.com/Default.aspx?ID=820>.

41. Raffel, M., "Particle Image Velocimetry: A Practical Guide,” Heidelberg: Springer, 2007.

42. "Ruby Laser," 15 Jan. 2012 $<$ http://physicsanimations.com/Physics/English/las_txt.htm>.

43. Bani Younes, A., H., "Investigation of the Flowfield Surrounding Small Photodriven Flapping Wings," Diss. University of Dayton, Dayton, OH, 2009.

44. UCLA Example Final Design Report, MAE 154, June 1, 2009.

45. “Airfoil Investigation Database,” 25 February 2012, 15 February 2012 $<$ http://www.worldofkrauss.com/about $>$.

46. Birch, D. M., "Self-Similarity of Trailing Vortices," University of Surrey, Guildford, Surrey, United Kingdon, 2012.

47. "Efunda Fluid Mechanics: Glossary," 15 April 2011

$<$ http://www.efunda.com/formulae/fluids/glossary.cfm>.

48. “Glossary of Meteorology," $2^{\text {nd }}$ ed., 1994, 15 April 2011 $<$ http://amsglossary.allenpress.com/glossary/search?id=inviscid-fluid1>.

49. Denton, R. E., Hu, Y., "Symmetry Boundary Conditions," Journal of Computational Physics, 2009.

50. Cellier, F. E., Kofman, E., “Continuous System Simulation,” Springer Science + Business Media, Inc. 2006. 


\section{APPENDIX A}

\section{ADDITIONAL PIV INFORMATION}

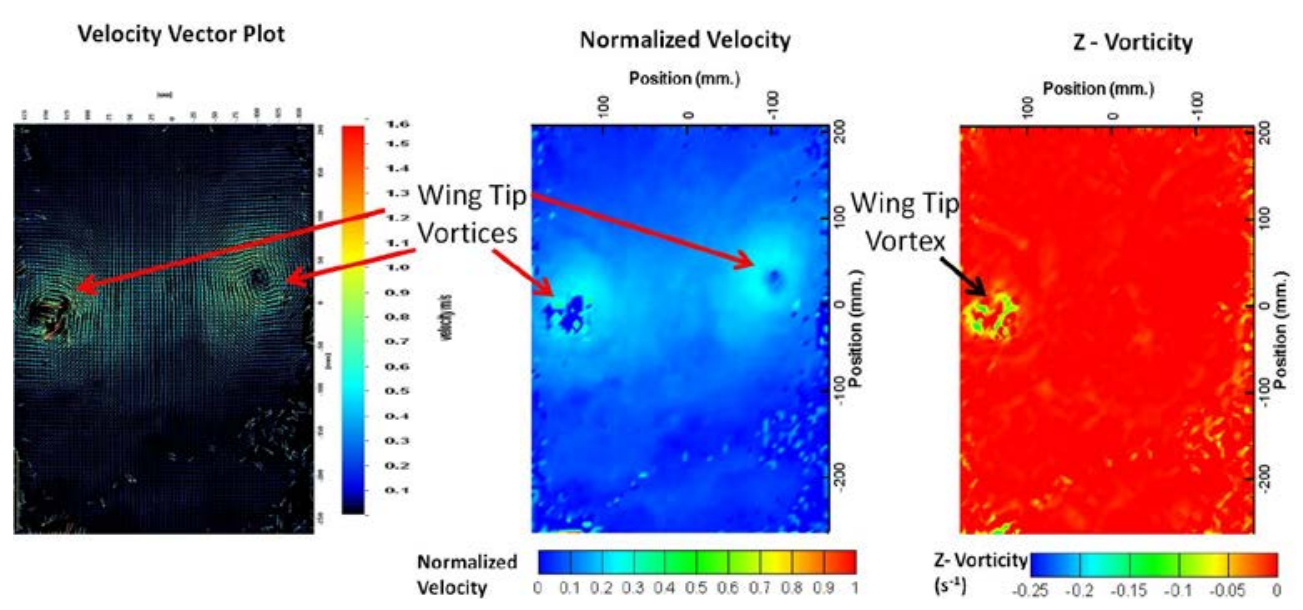

Figure A.1: Plots of the wingtip vortices from a fast straight flight without the wall.

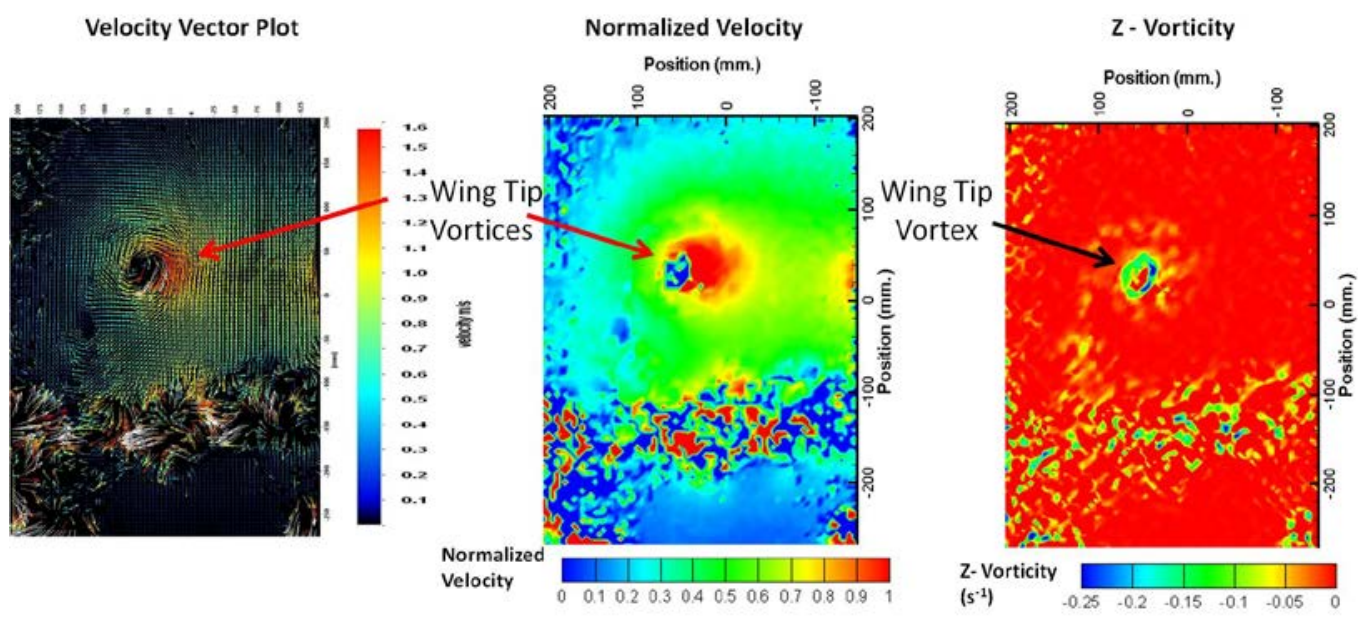

Figure A.2: Plots for a wingtip vortex from a slow straight test without the wall. 

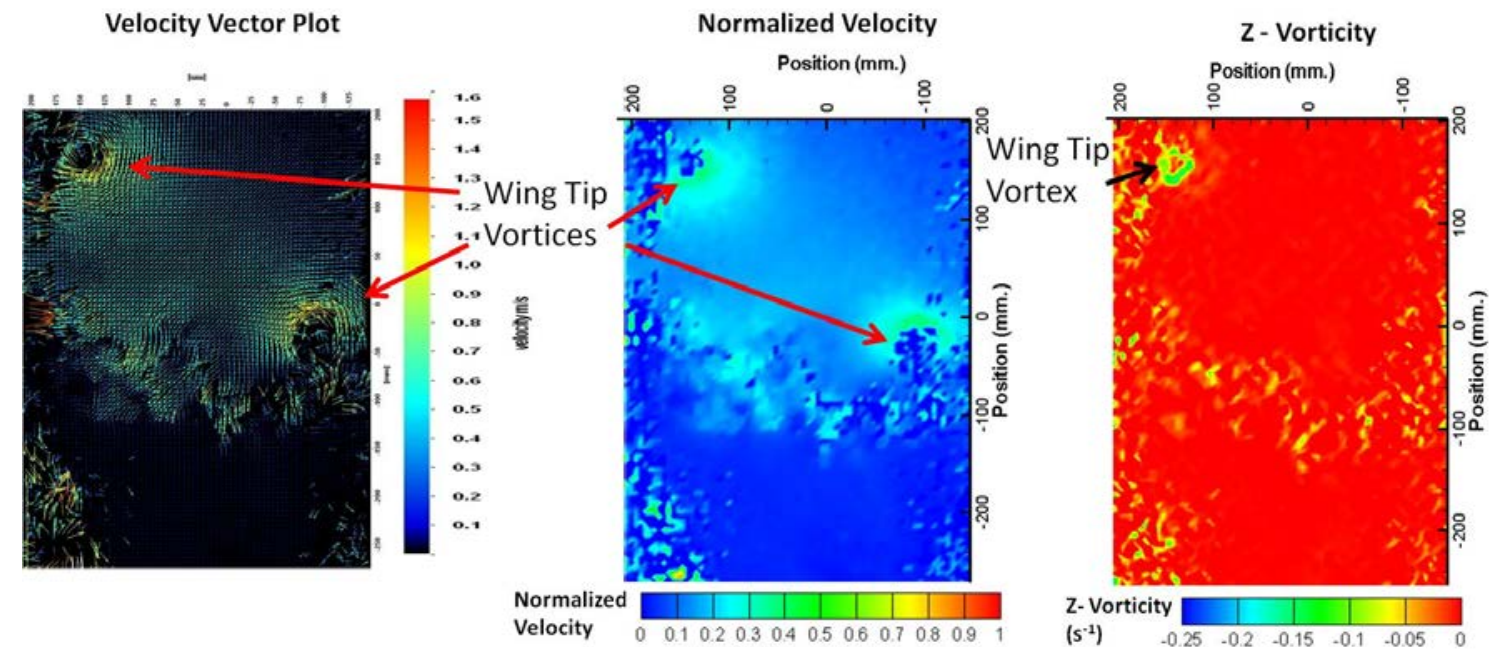

Figure A.3: Velocity vector field plot, normalized velocity contour, and zvorticity contour plot of the wingtip vortices from a fast turning flight without the wall.
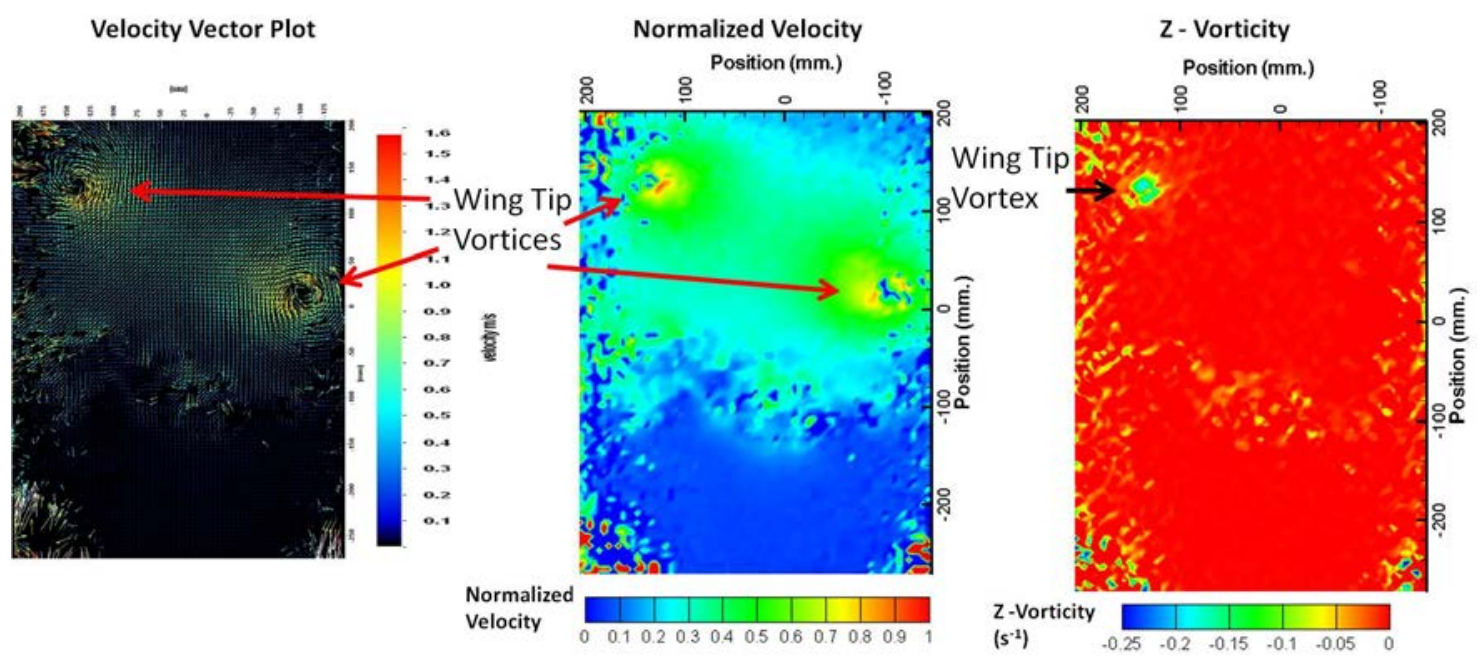

Figure A.4: Velocity vector field plot, normalized velocity contour, and zvorticity contour plot of the wingtip vortices from a slow turning flight without the wall. 


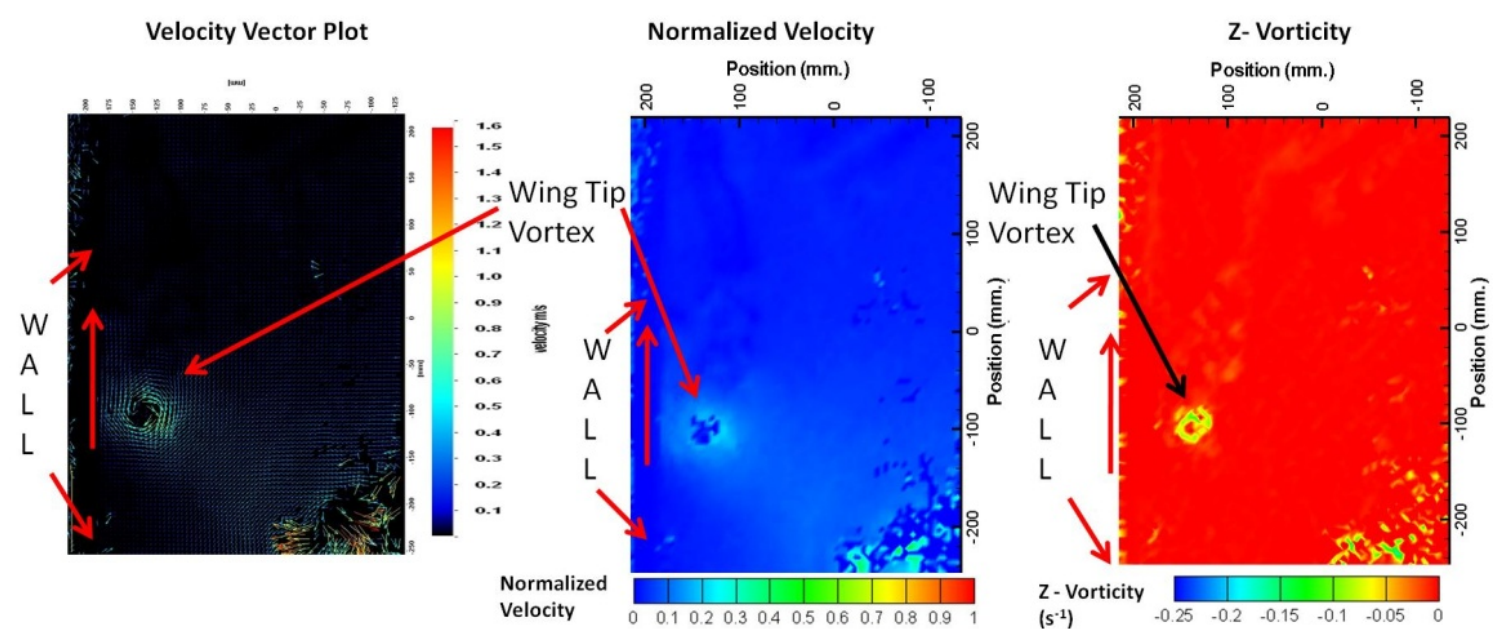

Figure A.5: Velocity vector field plot, normalized velocity contour, and zvorticity contour plot of the wingtip vortex from a fast straight flight along the wall.

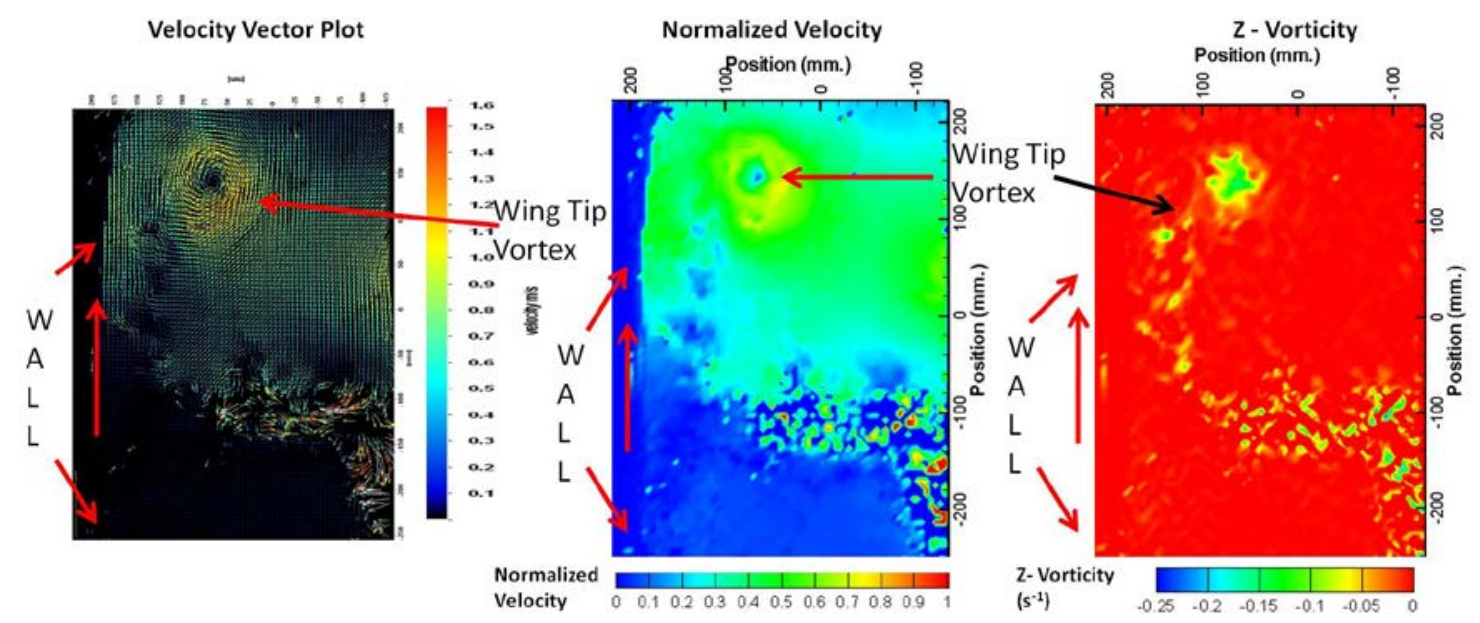

Figure A.6: Velocity vector field plot, normalized velocity contour, and zvorticity contour plot of the wingtip vortex from a slow straight flight along the wall 

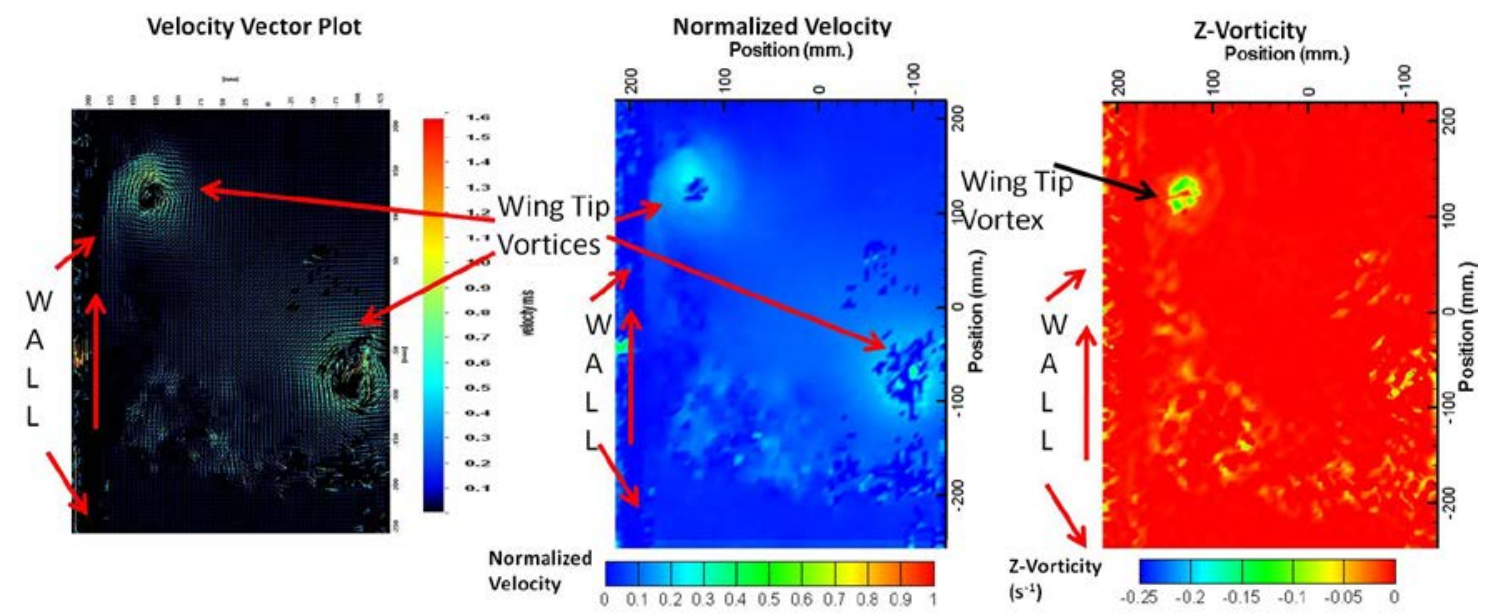

Figure A.7: Velocity vector field plot, normalized velocity contour, and zvorticity contour plot of the wingtip vortex from a fast turning flight along the wall.

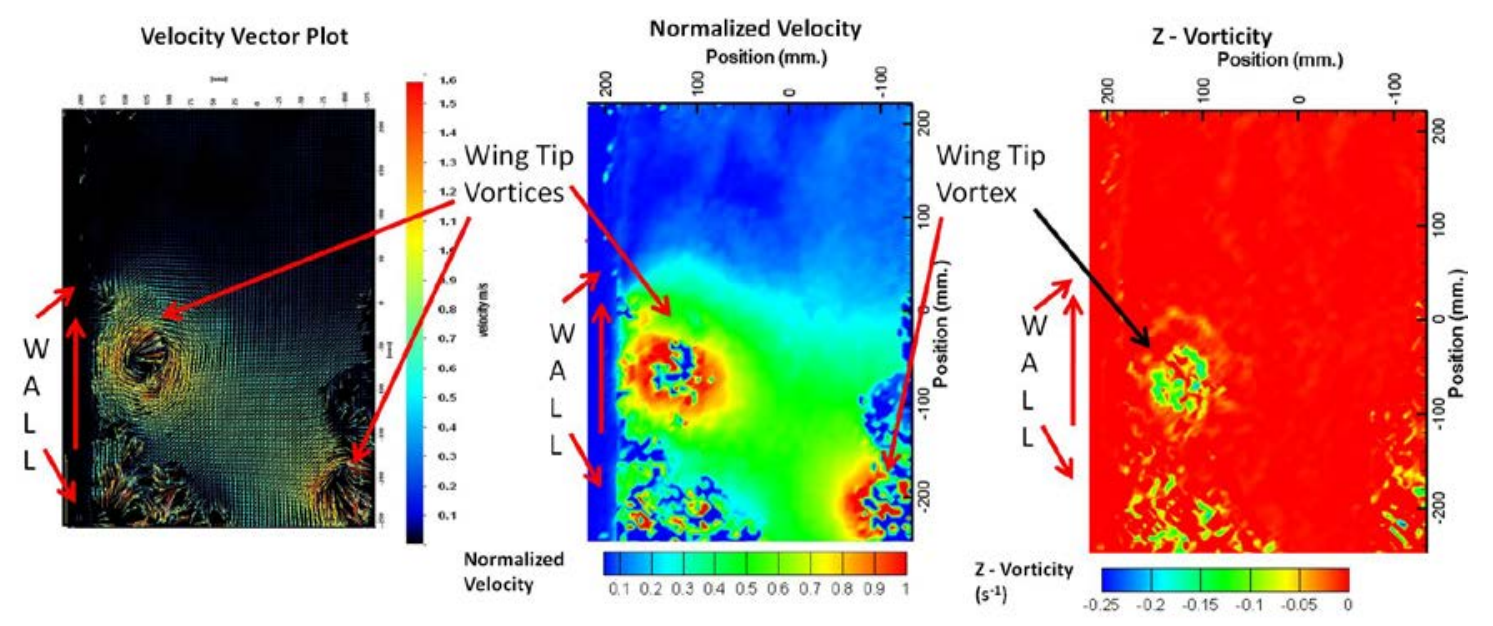

Figure A.8: Velocity vector field plot, normalized velocity contour, and zvorticity contour plot of the wingtip vortex from a slow turning flight along the wall. 

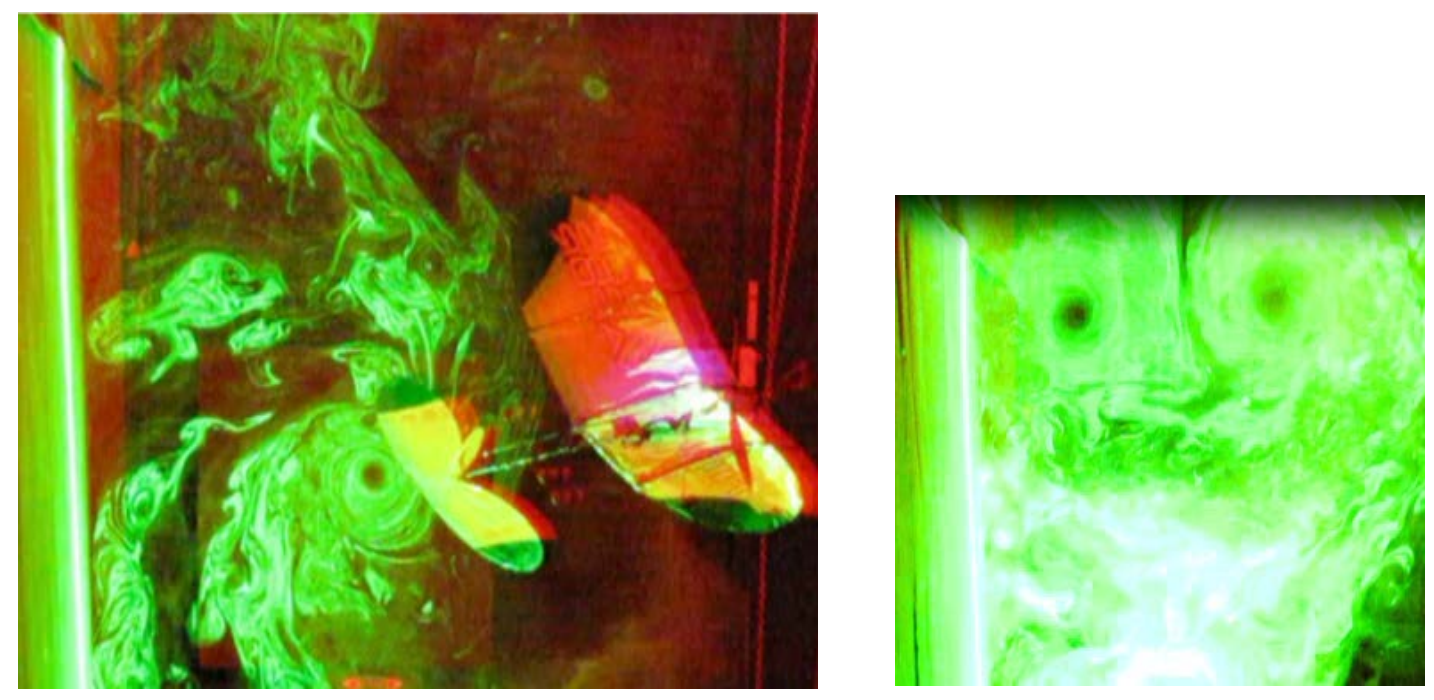

Figure A.9: Wingtip vortices are seen in these proof of concept testing images as the Night Vapor leaves the field of view. The PIV seeding is not ideal for analysis but clearly shows the formation of wingtip vortices.

Additional attempts at normalizing the circulation of the wingtip vortex data collected during the PIV testing is discussed next. As stated in the results in Chapter six of this thesis, little difference is noticed in the circulation values for the tests conducted with or without the wall present. It is thought that through normalizing the circulation values, a difference in circulation between these two types of tests will be made clear. First, the tests without the wall present are normalized by the vortex core diameter and the tests with the wall present are normalized by the distance between the vortex core and the wall. The vortex core diameter is an indicator of the strength of the vortex. The distance between the vortex core and the wall is important because a vortex has an influence on a body or flow as a function of the distance between the vortex and the body or flow. However, these normalization attempts do not help to reveal an aerodynamic difference in the circulation values between the tests. The average vortex core diameter without the wall is only $4.89 \mathrm{~cm}$ and the average distance between the vortex core and the wall is $8.82 \mathrm{~cm}$ for the tests with the wall present. Therefore, the normalized circulation 
of the wingtip vortices near a wall will always be lower than the normalized circulation of the tests without the wall. This is due to the fact that the average distance between the wall and the vortex core is roughly double the average vortex core diameter for the tests conducted without a wall present. Figure A.10 demonstrates this non aerodynamic related difference between the circulation values for the tests conducted with and without the wall present. The theoretical circulation for a fast straight test without the wall present is calculated and plotted by using the Kutta-Joukowski theorem and assuming that the lift of the vehicle is equal to the weight of the vehicle.

\section{Circulation of Wingtip Vortex from PIV Tests: Fast Straight Without Wall Tests Normalized by Vortex Core Diameter, Frast Straight with Wall Tests Normalized by Distance Between Wall and Vortex}

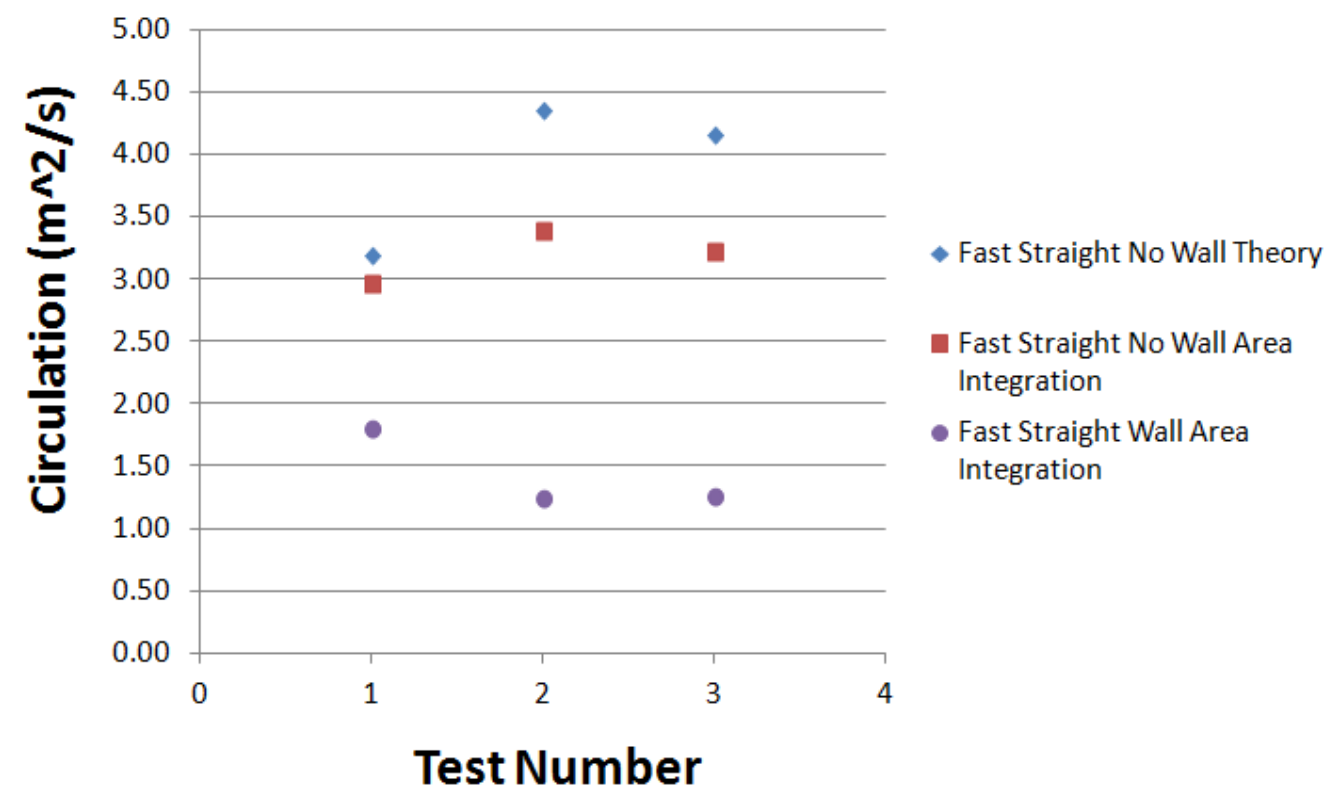

Figure A.10: Normalized circulation values for three different fast straight tests with and without the wall present. 
The following plots are additional graphs that use the same circulation normalization process discussed for Figure 6.4 in section 6.2.2.

Normalized Circulation Profiles for Slow Straight Tests

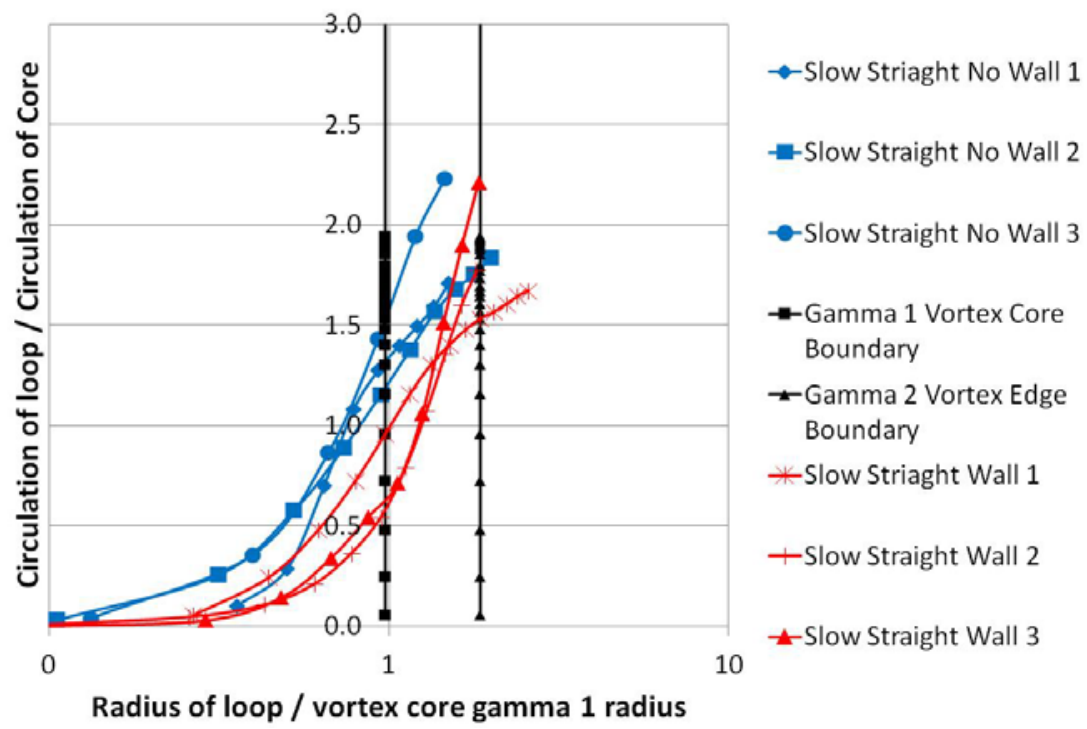

Figure A.11: Normalized circulation profiles for three slow straight tests with and without the wall present.

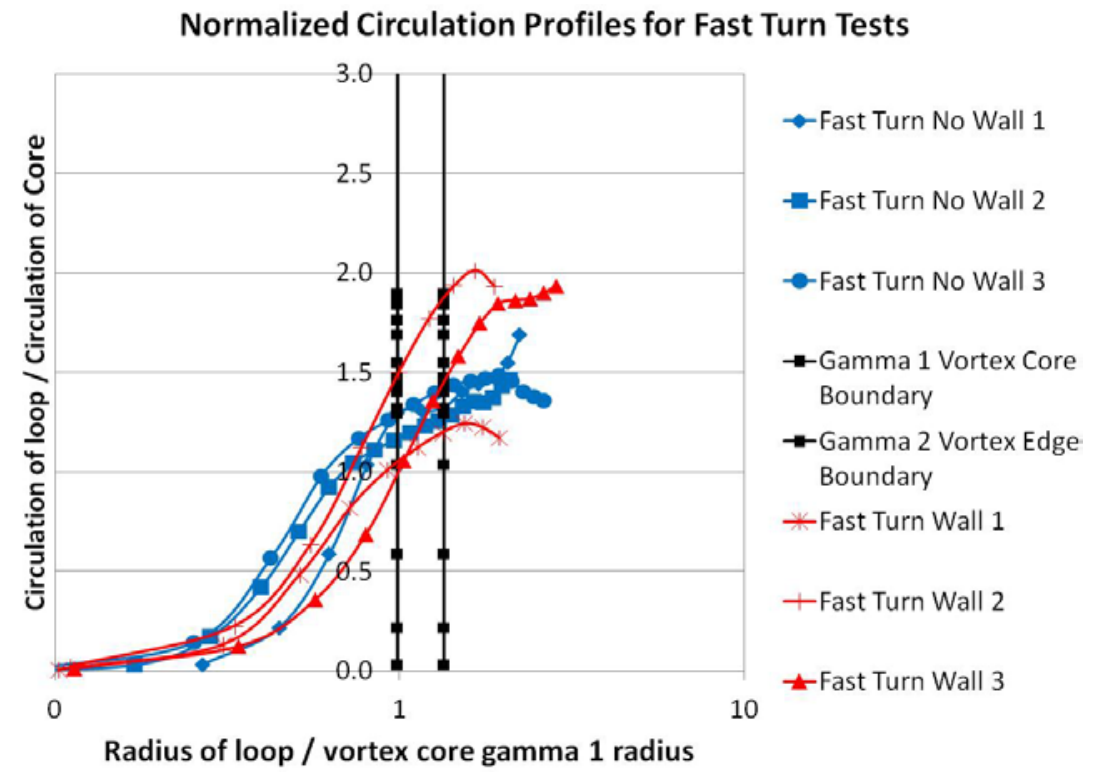

Figure A.12: Normalized circulation profiles for three fast turning tests with and without the wall present. 


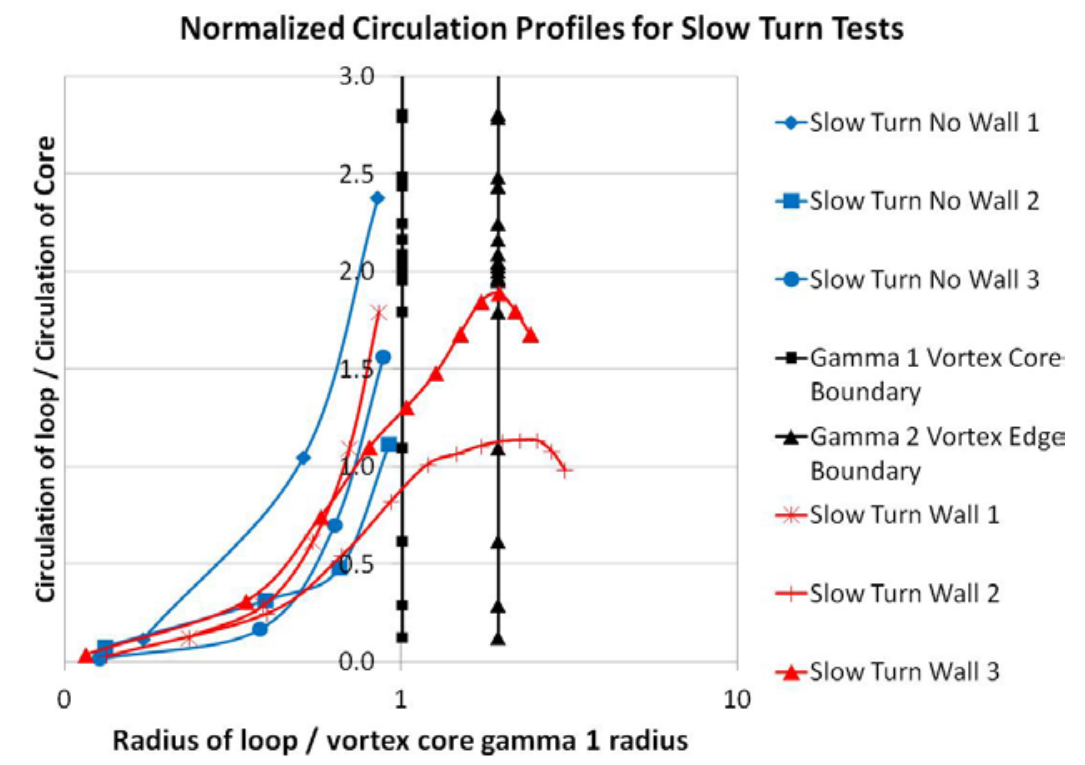

Figure A.13: Normalized circulation profiles for three slow turning tests with and without the wall present.

Figure A.14a shows the slow turn test two without the wall z-vorticity contour plot. This test has the highest normalized circulation values if the noisy z-vorticity around the vortex is included in the area integration circulation sensitivity analysis as seen in Figure A.15. Figure A.14b shows the z-vorticity contour plot of the slow turn test three near a wall. This test does not have a significant amount of spurious z-vorticity present in the outer layers of the area integration boxes compared to Figure A.14a. This lack of spurious z-vorticity in Figure A.14b helps this slow turn test along the wall to have normalized circulation values that are reasonable (see Figure A.15) compared to the literature of Birch [46] as discussed in section 6.2.2. 


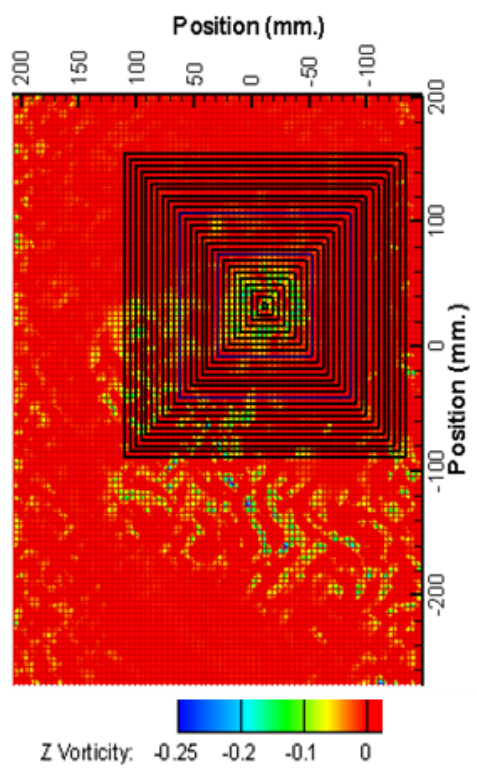

Figure A.14a: Slow turn test two without wall z-vorticity contour plot. The large amount of spurious z-vorticity seen in this image contributes to the larger normalized circulation values seen in Figure A.15.

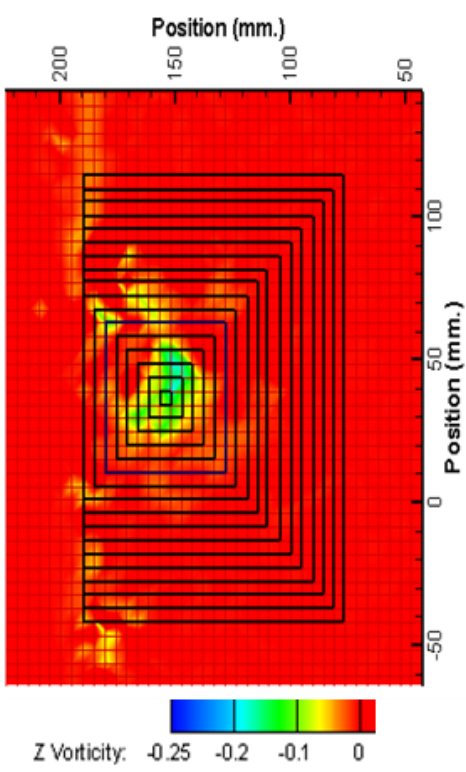

Figure A.14b: Slow turn test three with the wall present. Spurious z-vorticity is not seen in this image and the normalized circulation values are similar to the literature. See Figure A.15.

Figure A.15 shows a normalized circulation profile for the slow turn tests with the integration loops containing sporadic noisy z-vorticity included in the sensitivity analysis. The process of establishing a cutoff point for this data through looking at the corresponding images such as Figure A.14 a and Figure A.14 b, is discussed in section 6.2.2 of this thesis. 


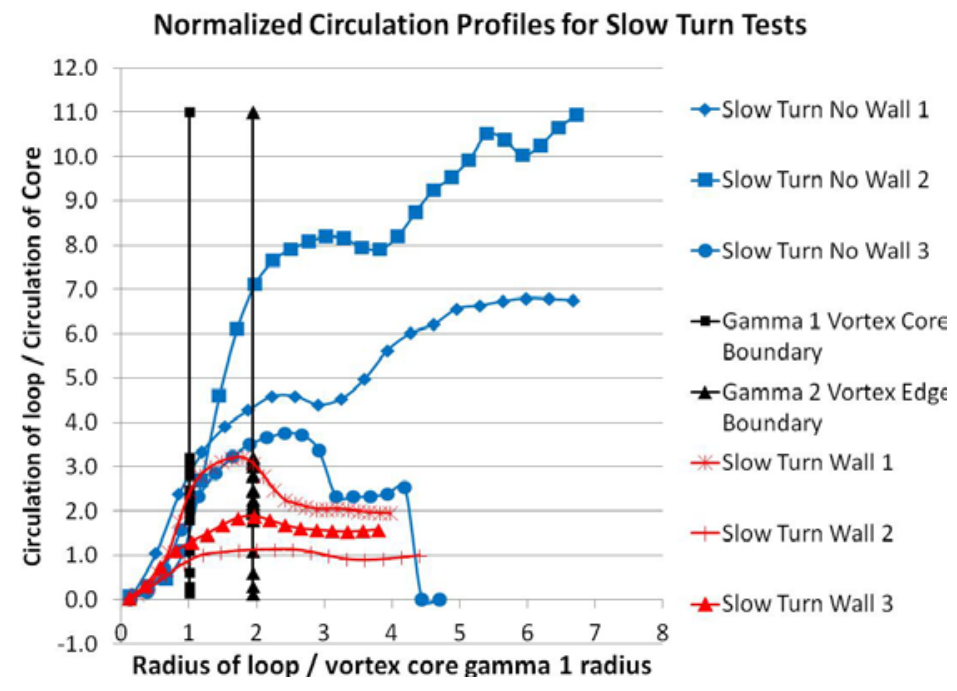

Figure A.15: Sporadic noisy z-vorticity causes unreasonable spikes in the normalized circulation of these slow turning test circulation profiles.

Finally, it is important to note the calculations that contribute to the uncertainty associated with the PIV testing.

- Uncertainty associated with measuring the distance and time of smoke particles moving during the PIV testing

- Uncertainty for taking the derivative to get velocity

- Uncertainty for taking the curl of the velocity field to get vorticity

- Uncertainty for taking the integral of the area of the z-vorticity values or the integral over the closed contour line of the velocity values to get the circulation values 


\section{APPENDIX B}

\section{POTENTIAL FLOW MODEL}

\section{B.1 Potential Flow Model Simulation Theoretical Background}

In order to begin to create a computer simulated model for a wingtip vortex near a wall, several assumptions for the flow under consideration must first be identified. In this analysis it will be assumed that the flow is irrotational, incompressible, two-dimensional, and inviscid. An irrotational fluid flow is a flow where the streamlines never loop back on themselves [47]. Mathematically, a flow is irrotational if the curl of the velocity vector goes to zero [34]. The continuity equation leads to the incompressible assumption. "An incompressible fluid is a fluid whose elements cannot experience volume change" [34]. Since the mass of a fluid element is constant by definition, the fluid elements of an incompressible fluid must have a constant density [34]. The continuity equation for an incompressible fluid goes to zero. Since this research will focus on a two-dimensional analysis, the third component of the vector field goes to zero. An inviscid fluid, also called an ideal fluid or perfect fluid, is a nonviscous fluid. "A nonviscous fluid is a fluid in which all surface forces exerted on the boundaries of each small element of the fluid act normal to these boundaries" [48]. 
In this research, both an analytical and a numerical solution are identified in order to model the wingtip vortex along a wall. These two solutions are modeled over every point within a mesh. This mesh is defined as the field of view from the PIV testing and has four boundary conditions. The left vertical boundary condition is defined as the wall that the vortex will be modeled along. The analytical and numerical solutions are compared against one another in order to validate each other. The analytical solution is first modeled without a free stream velocity in order to simplify the model; however, the user has the option to incorporate a free stream velocity into the analysis. If desired, this would help to simulate the proof of concept testing when the vortex traveled upwards due to a vertical free stream flow. This free stream velocity is added onto the analytical solution of a stream function for a vortex near a wall. This is possible through the superposition principle. The analytical velocity components can then be calculated by taking the derivative of the stream function. The magnitude of the velocity will then be calculated.

Laplace's equation is used to calculate the numerical solution for the stream function in every interior point within the mesh. The boundary conditions are carefully considered and are not trivial in this part of the model. First the stream function for a vortex located above a wall is used to define the four boundaries of the mesh. This equation is rotated $90^{\circ}$ to model a vortex to the right of a vertical wall. The analytical solution can also be applied as the boundary condition at the wall for the numerical solution. Although, this is not good practice, the problem is simplified in order to make the problem feasible. A symmetry boundary condition is also provided as an option to the user of the model. In this symmetry boundary condition, the stream function values 
along the wall will be initialized and then updated later after the interior stream function values have been calculated. If either the stream function or the symmetry boundary conditions are applied to the wall boundary condition in this numerical solution, the stream function in the corners must still be defined separately. The stream function for a vortex near a wall is applied to the corner stream functions in the mesh. Once the numerical solution has been calculated throughout the entire mesh, the numerical velocities are calculated next. All of the interior velocities can be calculated with the same second order central difference formula, but care must be taken when calculating the velocities along the boundaries of the mesh and the corners of the mesh. Second order biased formulas must be used according to each boundary or corner's geometry. Once all of the velocities at each point in the mesh are known, it is then possible to calculate the magnitude of the numerical velocities. The numerical and analytical stream functions and velocities are plotted and compared.

\section{B.2 Potential Flow Model Experimental Setup}

\section{B.2.1 Analytical Solution}

The analytical solution is calculated through using the equation for the stream function, $\psi$, for a vortex located to the right of a vertical wall. See Equation B.1.

$$
\psi=\frac{\Gamma_{\mathrm{z}}}{4 * \pi} * \ln \left[\frac{\left(y^{2}+(x-a)^{2}\right.}{\left(y^{2}+(x+a)^{2}\right.}\right]
$$

The variable "a" is defined as the distance from the wall to the center of the vortex and $\mathrm{x}$ and $\mathrm{y}$ are the Cartesian coordinate values of each point in the mesh. $\Gamma_{\mathrm{z}}$ is the 
circulation of the vortex, which is set to one in this model in order to simplify the equations.

If a vertical free stream is to be modeled in the code along with the vortex stream function for the vortex near a wall, the superposition principle is used and the two stream functions are added together. Again, this option is available since the proof of concept testing saw a free stream velocity in the vertical direction. See Equation B.2 for the stream function of a vortex near a wall with a vertical free stream added.

$$
\psi=\frac{\Gamma_{\mathrm{z}}}{4 * \pi} * \ln \left[\frac{y^{2}+(x-a)^{2}}{y^{2}+(x+a)^{2}}\right]+\mathrm{V}_{\infty} \mathrm{x}
$$

The analytical velocities in the $\mathrm{x}$ and $\mathrm{y}$ direction for the vortex without a free stream flow can be found through taking the derivative of Equation B.1. See Equation B.3 to see how the $u$ and $v$ velocities are derived from partial derivatives of the stream function. Maple software can be used to solve these partial derivatives for the $\mathrm{u}$ and $\mathrm{v}$ velocities for the derivatives of Equation B.1. The Maple code can be seen below in Equations B.4 a-c. In equation B.4a, equation $\mathrm{g}$ is the same as $\psi$ in Equation B.1. The variable $b$ represents the circulation. Equation B. $4 b$ is the partial derivative of $g$ with respect to the variable $\mathrm{x}$ and represents the $\mathrm{v}$ component of velocity. Equation $\mathrm{B} .4 \mathrm{c}$ is the partial derivative of $g$ with respect to the variable $y$ and represents the $u$ component of velocity. These $u$ and $v$ velocities are found for each point in the mesh for the analytical solution velocities.

$$
u=\frac{\partial \psi}{\partial y} \quad v=-\frac{\partial \psi}{\partial x}
$$




$$
g^{\prime}=\left(\frac{b}{(4 \cdot \mathrm{Pi})}\right) \ln \left(\frac{\left(y^{2}+(x-a)^{2}\right)}{\left(y^{2}+(x+a)^{2}\right)}\right)
$$

$D g x:=\operatorname{diff}(g, x)$

$$
\frac{1}{4} \frac{b \ln \left(\frac{y^{2}+(x-a)^{2}}{y^{2}+(x+a)^{2}}\right)}{\pi}
$$

$D g y:=\operatorname{diff}(g, y)$

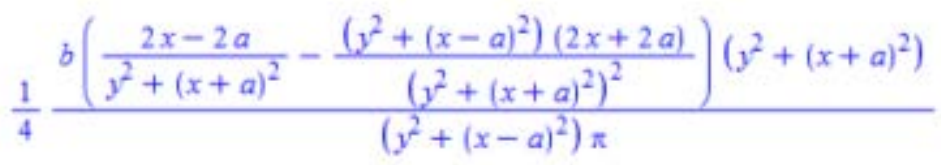

$$
\frac{1}{4} \frac{b\left(\frac{2 y}{y^{2}+(x+a)^{2}}-\frac{2\left(y^{2}+(x-a)^{2}\right) y}{\left(y^{2}+(x+a)^{2}\right)^{2}}\right)\left(y^{2}+(x+a)^{2}\right)}{\left(y^{2}+(x-a)^{2}\right) \pi}
$$

The magnitude of the velocity is found through taking the square root of the sum of the squares of the $u$ and $v$ velocities at each point in the mesh.

\section{B.2.2 Numerical Solution}

For the numerical solution, the left wall, bottom, top, and right side boundary conditions of the field of view mesh are defined by the analytical stream function in Equation B.1. If the symmetry boundary condition is to be applied to the left side wall boundary condition, the other boundaries still have the same stream function boundary condition.

"Symmetric means that the field is even across the boundary; that is, $\mathrm{f}\left(\mathrm{x}_{0}\right.$ $+\Delta \mathrm{x})=\mathrm{f}\left(\mathrm{x}_{0}-\Delta \mathrm{x}\right)$. In finite difference simulations, boundary conditions are typically implemented using "buffer" or "ghost" grid points beyond the boundary. Then any centered finite difference formula for the first derivative at $\mathrm{x}=\mathrm{x}_{\mathrm{n}}$ will yield zero" [49].

For a symmetry boundary condition, the normal derivative to the wall is 0 .

Equation B.5 shows that a $2^{\text {nd }}$ order centered difference formula applied to the wall yields a value of zero. 
$2^{\text {nd }}$ order accurate formula for the first derivative at a grid point $\mathrm{j}$.

$$
\left(\frac{\partial \psi}{\partial x}\right)_{j=1}=\frac{\psi_{j+1}-\psi_{j-1}}{2 * \Delta x}=0
$$

This is true if $\psi_{j+1}=\psi_{j-1}$, which occurs if a symmetry boundary condition is present.

Since the above derivative is zero, the below derivative (Equation B.6) will also be zero. When we look at $\psi$ at the wall, a $2^{\text {nd }}$ order accurate forward biased formula can be used (Equation B.7) since it is necessary to calculate the values of $\psi$ at the left most boundary condition along the wall.

$$
\begin{array}{r}
\left(\frac{\partial \psi}{\partial x}\right)_{j=1}=\frac{1}{2 * \Delta x}\left(-\psi_{j+2}+4 \psi_{j+1}-3 \psi_{j}\right)=0 \\
\left(\frac{\partial \psi}{\partial x}\right)_{j=1}=\frac{1}{2 * \Delta x}\left(-\psi_{3}+4 \psi_{2}-3 \psi_{1}\right)=0
\end{array}
$$

Solve for $\psi_{1}$ which is the value of the stream function at the wall that needs to be solved with this symmetry boundary condition (Equation B.8) [50].

$$
\psi_{1}=\frac{4}{3} \psi_{2}-\frac{1}{3} \psi_{3}
$$

Implement this equation for each $\psi$ value along the wall in the model. This equation will be used to both initialize the stream function values along the wall as well as update the stream function values along the wall after all the interior psi values have been solved. This completes the requirements for the numerical symmetry boundary condition if the user decides to implement this option.

Once the numerical boundary conditions are in place, the corners of the mesh must still be taken into account. The boundary conditions for the corners of the mesh will be defined by the stream function for a vortex near a wall, Equation B.1. 
Next, the numerical interior stream function values are calculated through solving the Laplace equation (Equation B.9) in two-dimensional Cartesian coordinates. "The Laplace equation is a statement of the incompressible continuity equation for an irrotational fluid" [34]. A $2^{\text {nd }}$ order centered difference for a $2^{\text {nd }}$ derivative (Equation B.10) is applied to each part of the Laplace equation in order to solve for $\delta \psi$ (Equation B.11) .

$$
\begin{gathered}
\frac{\partial^{2} \psi}{\partial \mathrm{x}^{2}}+\frac{\partial^{2} \psi}{\partial \mathrm{y}^{2}}=0 \\
\left(\frac{\partial^{2} \psi}{\partial x^{2}}\right)_{j}=\left(\delta_{x x} \psi\right)_{j}=\frac{1}{\Delta x^{2}}\left(\psi_{j+1}-2 \psi_{j}+\psi_{j-1}\right) \\
\delta \psi_{j, k}=\left[\frac{\left[\frac{\psi_{j+1, k}+\psi_{j-1, k}}{\Delta x^{2}}+\frac{\psi_{j, k+1}+\psi_{j, k-1}}{\Delta y^{2}}\right]}{\frac{2}{\Delta x^{2}}+\frac{2}{\Delta y^{2}}}\right]-\psi_{j, k}
\end{gathered}
$$

Once the numerical stream function is known, the numerical velocity components can be calculated for the interior nodes as well as the nodes along the left, right, top, and bottom boundaries. The numerical velocity components, both $\mathrm{u}$ and $\mathrm{v}$, for the interior nodes are calculated through using the $2^{\text {nd }}$ order central difference formula in Equation B.12.

$$
\left(\frac{\partial \psi}{\partial x}\right)_{j}=\frac{\psi_{j+1}-\psi_{j-1}}{2 * \Delta x}
$$

The numerical velocity components along the boundaries of the field of view and in the corners of the field of view are calculated through using a $2^{\text {nd }}$ order biased formula. This biased formula can be biased forwards or backwards depending on the location of interest. An example of a generic $2^{\text {nd }}$ order backward biased formula is seen in Equation B.13. 


$$
\left(\frac{\partial \psi}{\partial x}\right)_{j}=\frac{\psi_{j-2}-4 \psi_{j-1}+3 \psi_{j}}{2 * \Delta x}
$$

The magnitude of the numerical velocity components is calculated by taking the square root of the sum of the squares of the velocity components throughout the mesh.

In summary, the analytical and numerical potential flow model solutions serve as a tool to help investigate modeling techniques for a MAV wingtip vortex interacting with a wall.

\section{B.3 Potential Flow Model Procedure}

The degree of accuracy is an important aspect for analyzing the flow of a vortex near a wall. For the potential flow model it is important to remember that grids have to develop from a family of grids because this relates to the accuracy of the study. The boundary conditions and nonsmoothness can reduce theoretical orders of accuracy. Overall, the model is second order accurate.

In this model, a Cartesian mesh is developed and utilized. No inflation or other functions are used in the mesh and the $0.254 \mathrm{~m} \mathrm{X} 0.254 \mathrm{~m}$ overall dimension of the mesh is based on the field of view used during the proof of concept PIV testing described earlier. This scale gives a reference to the size of the mesh. Meshes consisting of 2,500, 10,000, and 40,000 nodes are also implemented and are discussed in the grid convergence study.

When the Laplace equation solves the numerical stream function for the interior nodes, the tolerance on the residual determines when the code converges. This residual is set as close to zero as possible in order to force the code to converge after the change in the stream function moves as close to zero as possible. The code continues to run until 
the residual is less than $1 * 10^{-12}$. This limit on the stream function is used since it would be unreasonable to expect the computer to have the capability to go beyond $1 * 10^{-15}$ or $1 * 10^{-16}$ for the machine zero.

The error between the analytical and numerical stream functions is determined to be $4.2733 \mathrm{e}-004$. This value is determined through using Equation B.14. Across the entire mesh, the differences between the corresponding numerical and analytical stream functions are calculated and the results are summed. Then the sum is divided by the number of mesh grid points and the square root of the result is calculated. The analytical and numerical stream functions seem to match up well since this error is small.

$$
\text { Error }=\sqrt{\frac{\sum_{1}^{N X}(\text { numerical-analytical })^{2}}{N}}
$$

The order of accuracy is also affected by several limitations associated with this approach. These limitations are identified at the beginning of the research project and the boundary conditions are identified as a significant challenge. The numerical and analytical solutions are calculated and compared, but it is impossible to define the boundary conditions of the numerical solution without already knowing the analytical solution and using it for the numerical solution boundary condition. This serves as a fundamental problem in the theory of this approach and will have a negative effect on the order of accuracy. The symmetry boundary condition also has issues. The value of the stream function along the wall during the symmetry boundary condition did not have a value of zero as it should. This means that a different solution is calculated and that the code did not produce the correct solution. This means that the solution is not unique. 


\section{B.4 Potential Flow Model Results}

The goal of the potential flow model is to investigate how a vortex interacts with a wall in a repeatable controlled computer model that can be compared to the PIV free flight tests. This will help to analyze how the wingtip vortex interacts with the wall and how the MAV flight controls are affected. When the model runs, the user defines if they would like to use a symmetry boundary condition or the stream function boundary condition along the wall. Next, the user can chose if they would like to superimpose a free stream flow into the vicinity of the vortex to model the proof of concept testing when the heated smoke particles forced the wingtip vortex to rise.

Figure B.1 shows the convergence plot for the norm of the residual vs. the number of iterations for the mesh with 2,500 nodes. It converged after about 240 iterations with the convergence criteria described earlier in this appendix in section B.3.

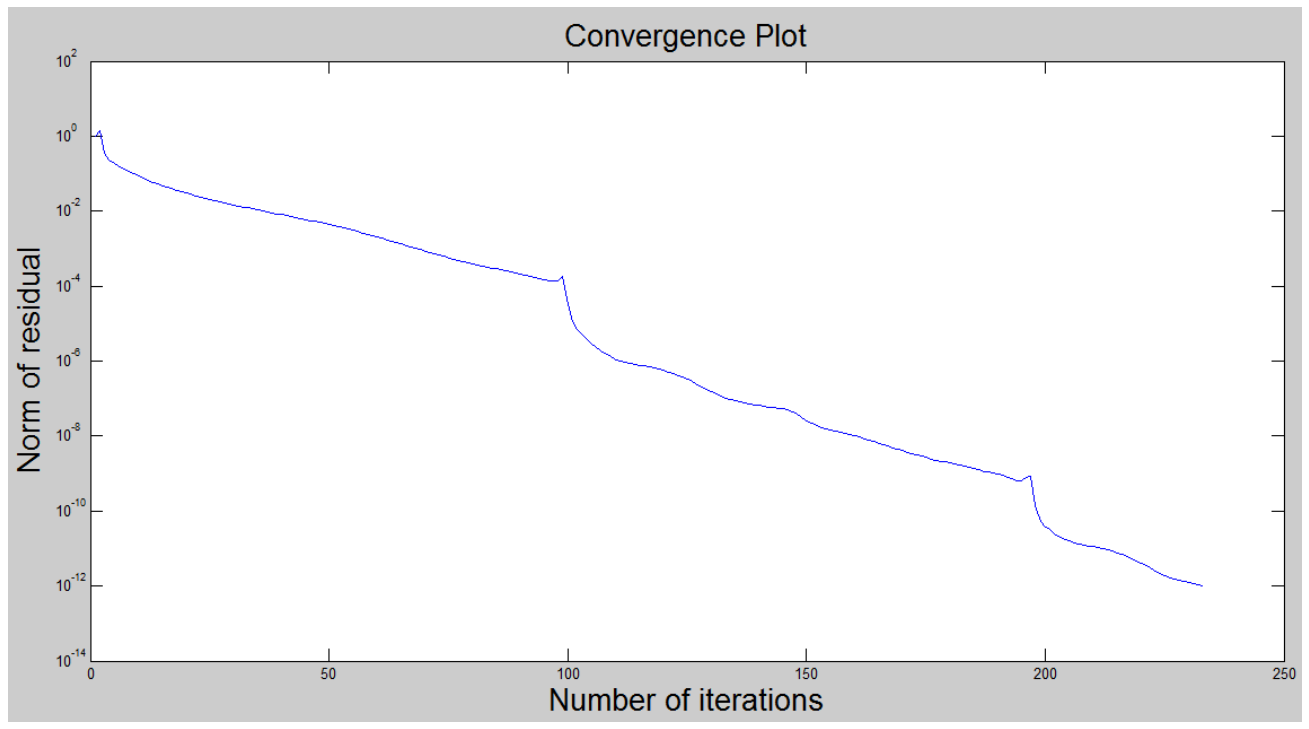

Figure B.1. Convergence plot for norm of residual vs. number of iterations. 
The contour plots of the numerical and the analytical stream functions can be seen in Figure B.2. The contour plots appear to be very similar to one another. The vortex is on the $\mathrm{x}$ axis since only a dimensional value away from the $\mathrm{y}$ axis is specified in the stream function in order to simplify Equation B.1. There is no free stream velocity imposed on this example and it uses a stream function boundary condition.

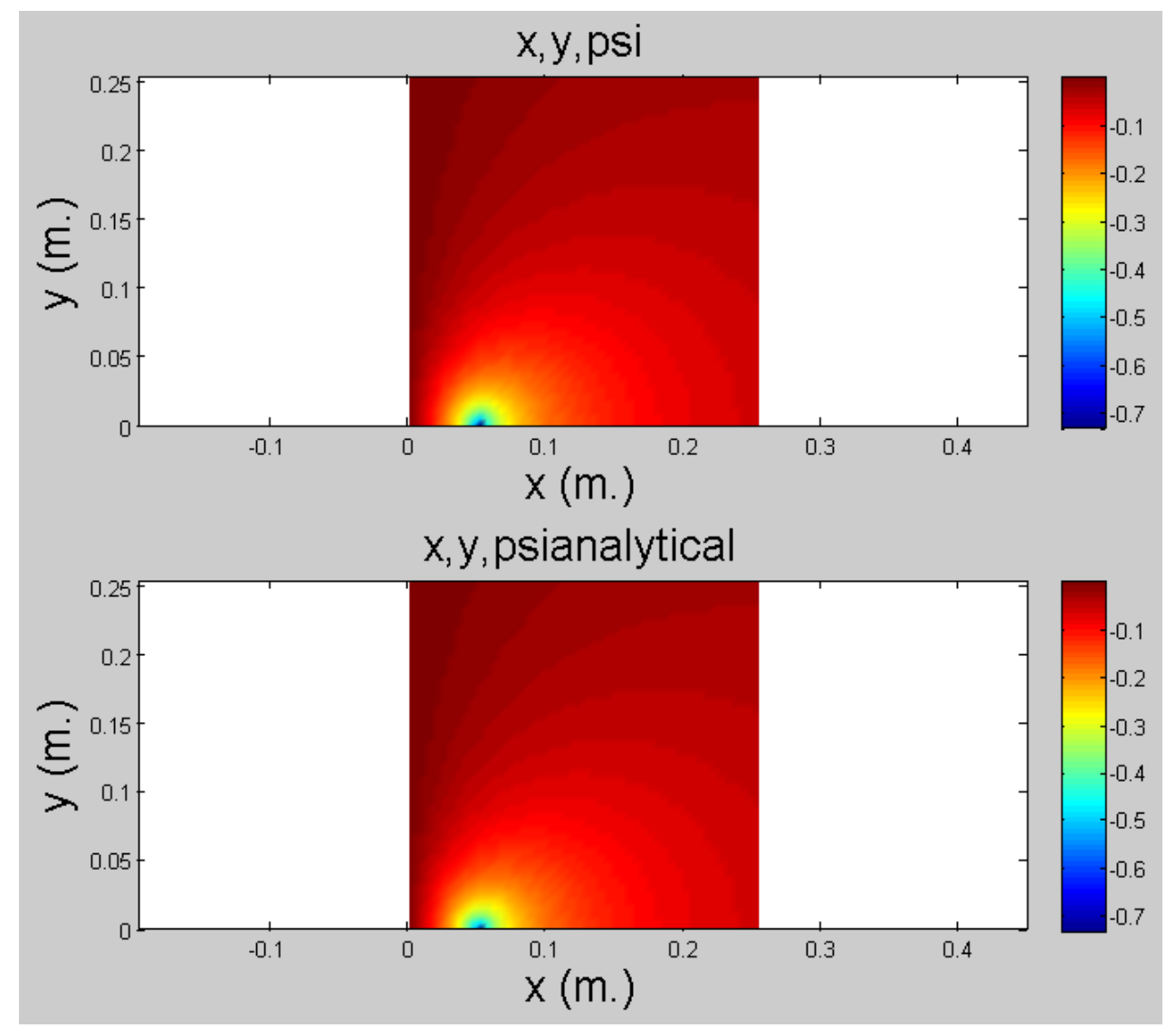

Figure B.2: Numerical and analytical stream function vortex contour plots look similar.

Figure B. 3 shows that the numerical and analytical stream function values are the same for several interior columns and rows in the mesh. This plot is produced with the stream function boundary condition at the wall and no free stream. 


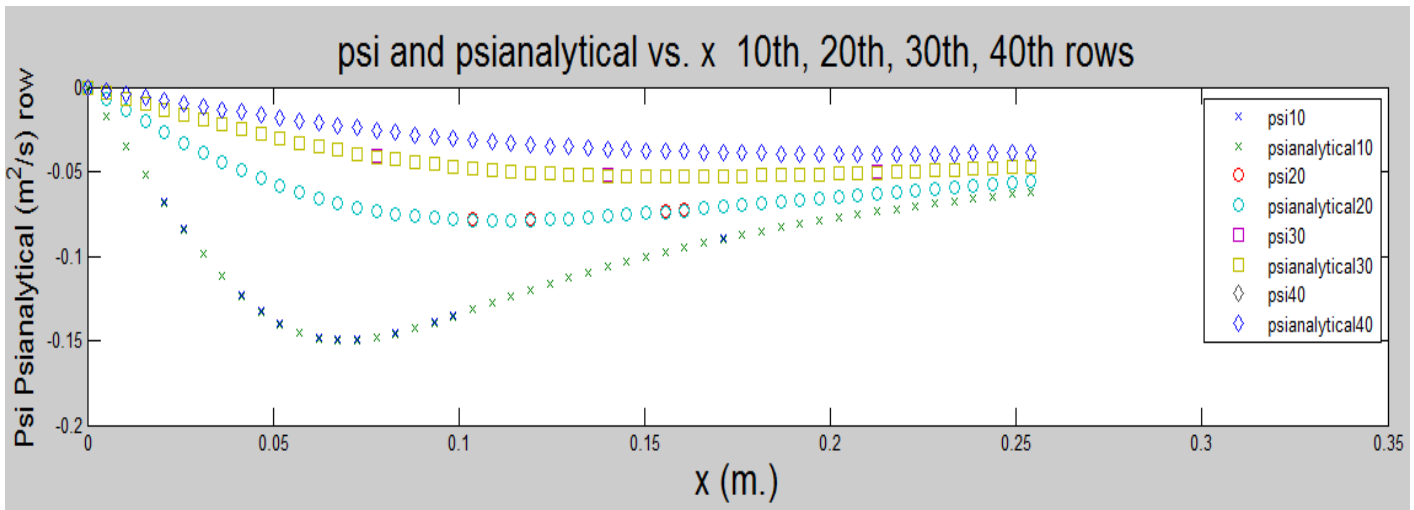

psi and psianalytical vs. y 10th, 20th, 30th, 40th columns

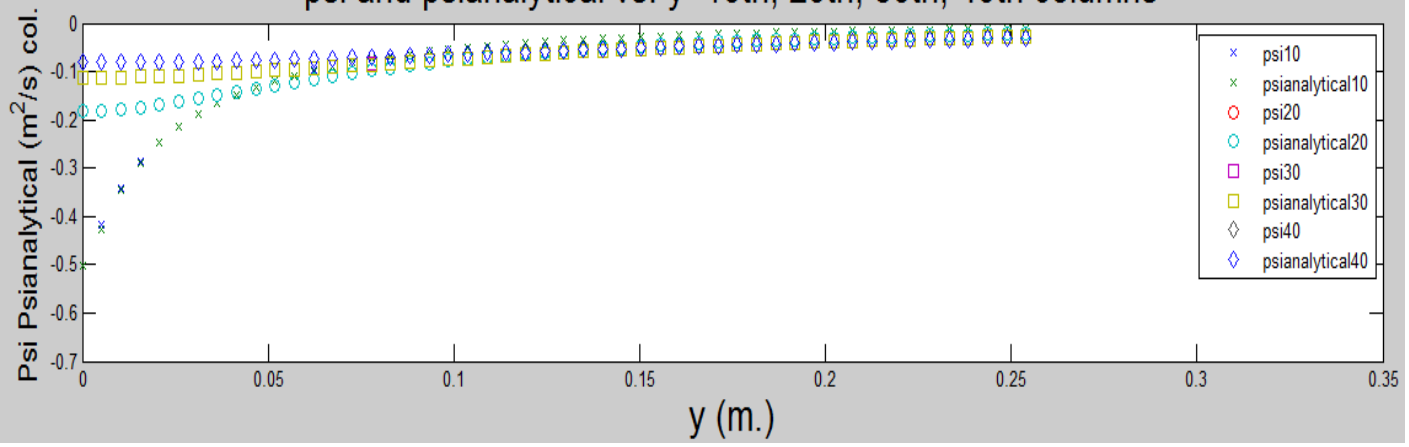

Figure B.3: Analytical and numerical stream function values for several interior rows and columns in the mesh.

Figures B.4a and B.4b show the effect of introducing a free stream flow into the analytical solution during an animated plot. A free stream velocity of two $\mathrm{m} / \mathrm{s}$ is used in order to provide clear differences between the two plots. The vortex with the superimposed free stream seems to be elongated compared to the vortex without the free stream. 


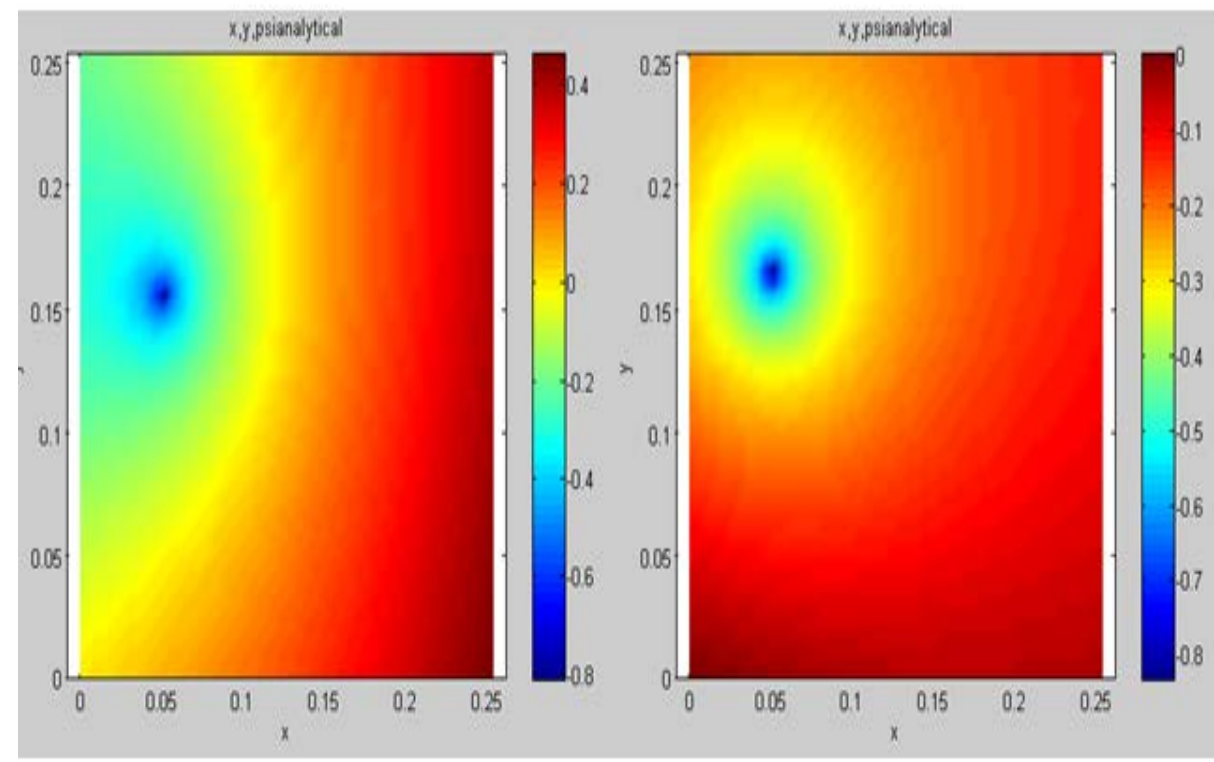

Figure B.4a: The stretched shape of the analytical stream function vortex contour plot is due to the added vertical free stream flow.
Figure B.4b: The circular shape of the analytical stream function vortex contour plot without the vertical free stream added can be seen.

While the analytical and numerical stream functions seem to be very similar, the magnitudes for the numerical and the analytical velocities also seem to be similar everywhere except for the wall boundary condition. Figure B.5 shows contour plots of the magnitudes of the velocity for the numerical and the analytical solutions. The left graph is focused on the entire field of view whereas the right figure is zoomed in on the vortex. It can clearly be seen that there is a discontinuity between the two and that the scale of the numerical solution is significantly higher than that of the analytical solution. This might be due to an error in the code since the stream functions give good results but the derivatives used to obtain velocity do not seem to match. 

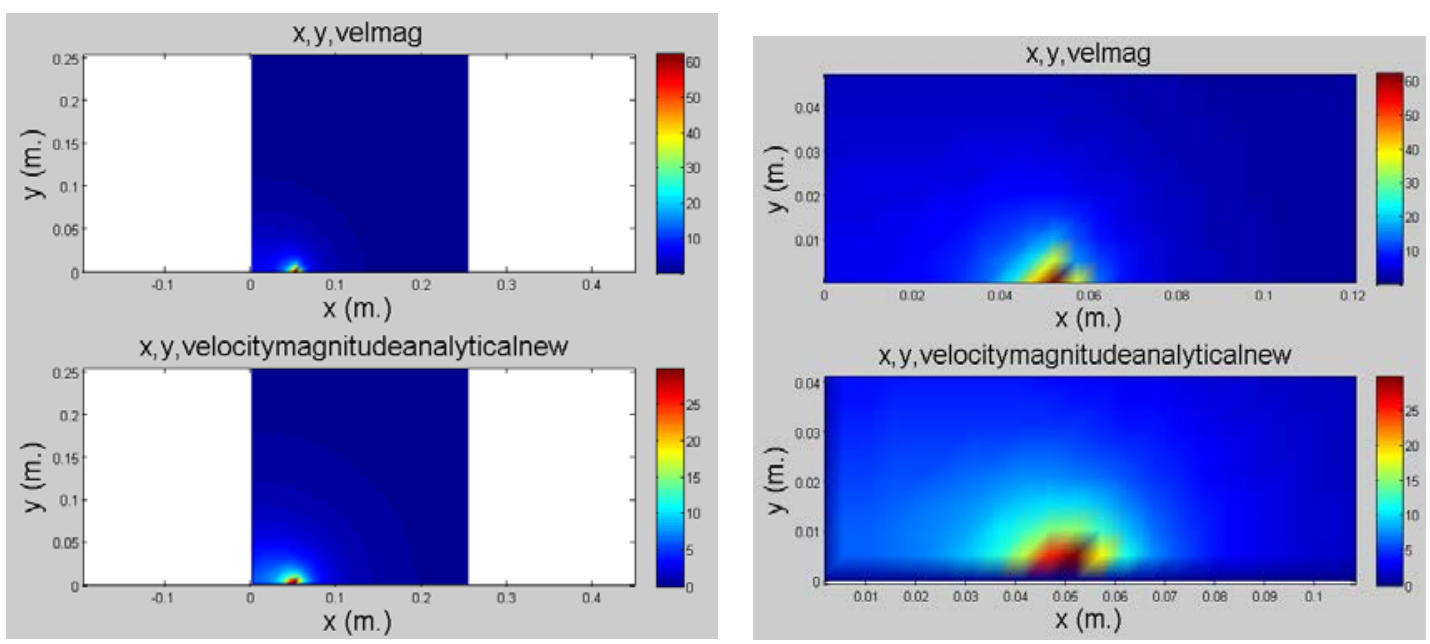

Figure B.5: $\mathrm{X}$ and $\mathrm{Y}$ position with the magnitude of the analytical and numerical velocities plotted as contours.

Figure B.6: shows a plot of the X position vs. the difference between the magnitude of the numerical velocities and the magnitude of the analytical velocities.

These velocities are derived by running the model without a free stream present and using the stream function boundary condition at the wall. Four different rows of the difference between the two velocity magnitudes are plotted. There seems to be no difference between the analytical and numerical velocity magnitudes, except at the boundary of the wall. This could be due to the stream function boundary condition. 


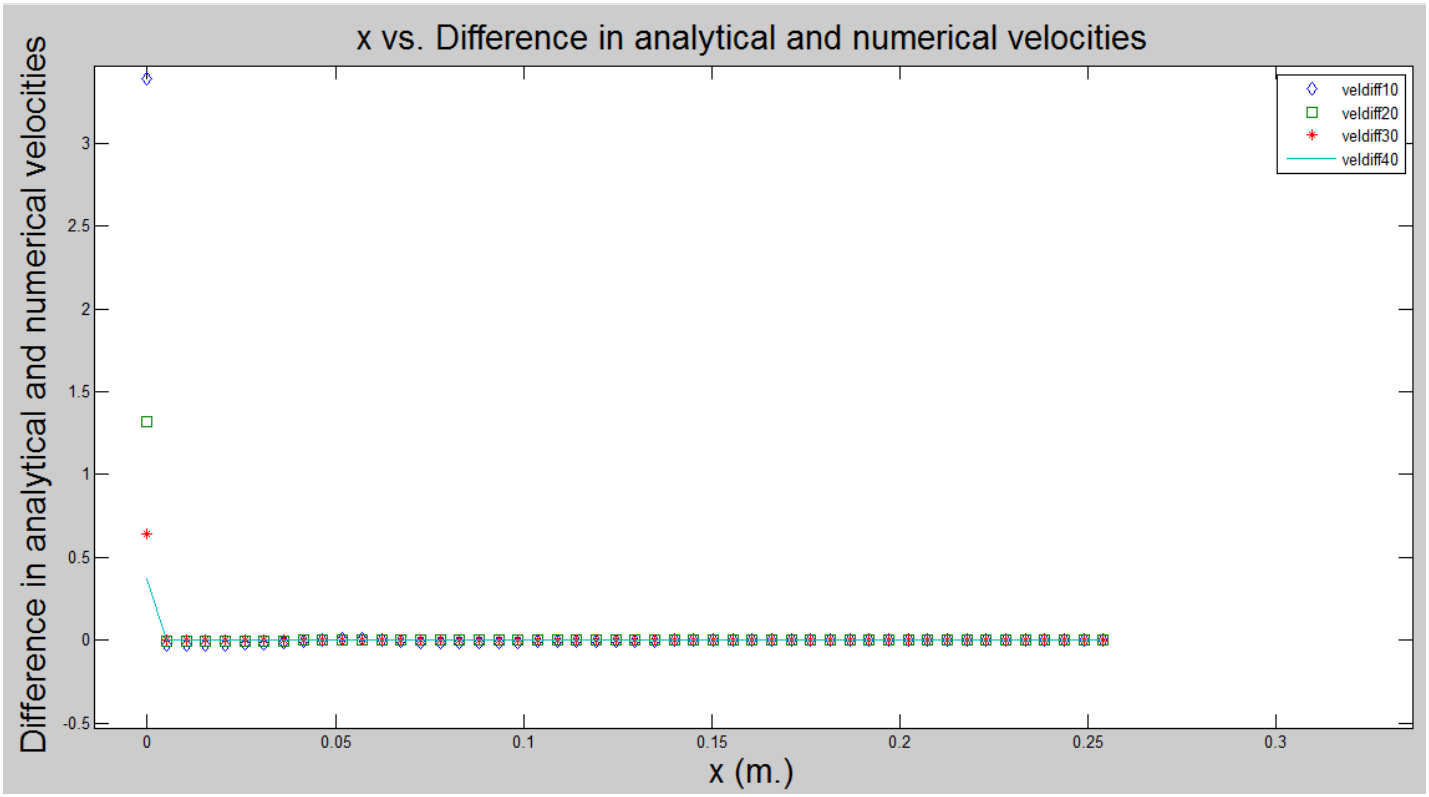

Figure B.6: X distance vs. the difference in the analytical and numerical velocity magnitudes.

\section{B.4.1 Grid Convergence Study}

The grid convergence study is conducted under the simplest test conditions: there is no free stream flow present and the wall boundary condition is fulfilled with the stream function for a vortex near a wall. The error in the stream function is used and the results of the grid convergence study can be seen below in Table B.1.

Figure B.7 displays the slope of the grid convergence trend line as -0.45 , which seems odd because one would expect the slope to be negative one since the code is second order accurate and is in two-dimensions. The stream function error seems to be correct since the analytical and numerical stream functions seem to match. However, the discontinuity may be involved with the fact that the problem is not a well posed problem and the potential flow approach is not the best method to use to solve this problem. 
Although the magnitude of the slope of the grid convergence line seems incorrect, the slope is still negative and the error reduces as the number of nodes increases.

Table B.1: Grid convergence results. The error reduces with more grid points.

\begin{tabular}{|c|c|c|c|c|}
\hline \multicolumn{5}{|c|}{ Grid Convergence Study Vortex Potential Flow Model: } \\
\hline NX & Number of Total Nodes & Error & Log(\# Nodes) & Log (Error) \\
\hline 50 & 2500 & 0.0004273 & 3.40 & -3.37 \\
\hline 100 & 10000 & 0.000231 & 4.00 & -3.64 \\
\hline 200 & 40000 & 0.000124 & 4.60 & -3.91 \\
\hline
\end{tabular}

Grid Convergence Study: Psi Error vs. Number of Nodes Using Potential Flow Wall Boundary Condition

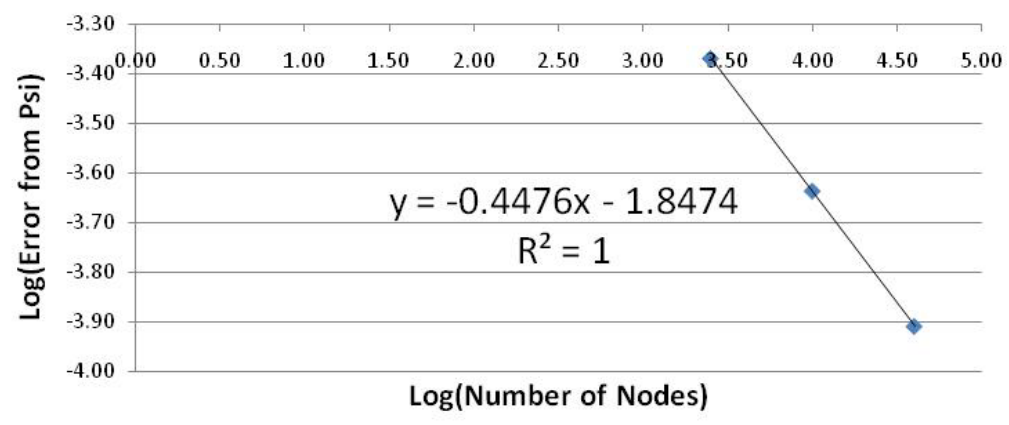

Figure B.7: Plot of the grid convergence study results with the slope of the trend line included.

Although the potential flow model approach taken in this thesis may not be a well posed problem and a unique solution was not obtained, a great amount of learning took place and it was a valuable experience overall. 